\title{
Strangeness Production \\ ON THE NUCLEON
}

\section{STIJN JANSSEN}

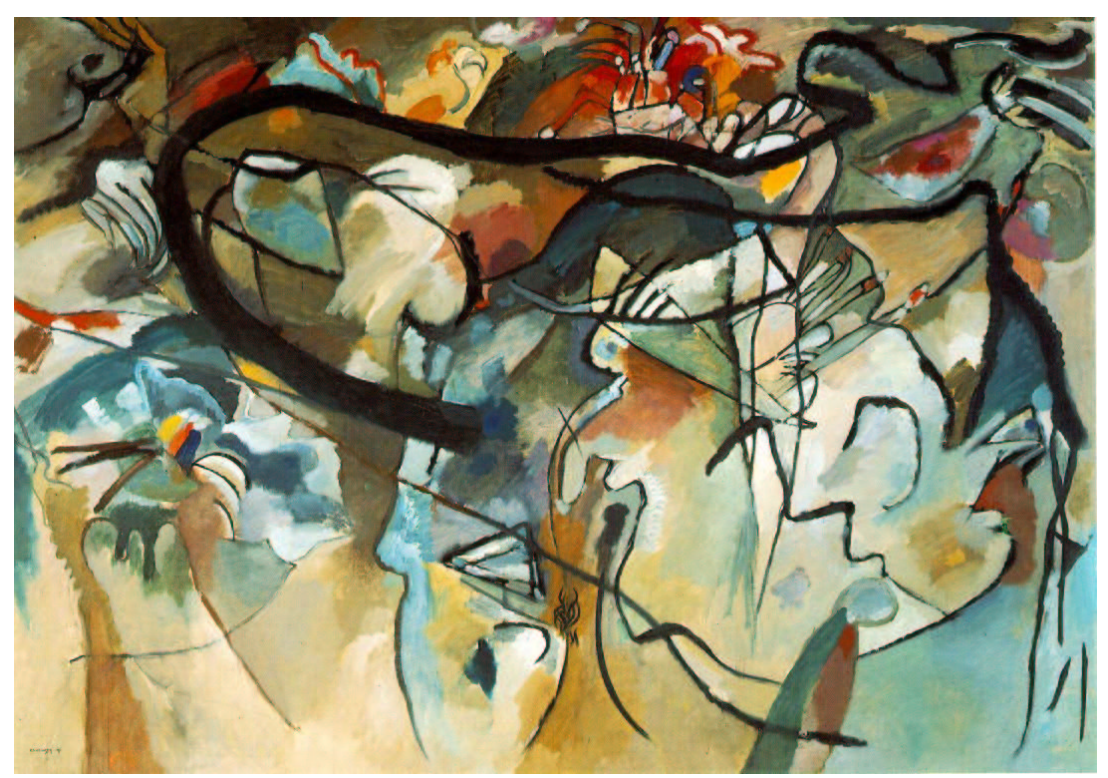

Promotor: Prof. dr. JAN RYCKEBUSCH

Proefschrift ingediend tot het behalen van de graad van

Doctor in de Wetenschappen: Natuurkunde

Universiteit Gent

Faculteit Wetenschappen

Vakgroep Subatomaire en Stralingsfysica

Academiejaar 2001-2002

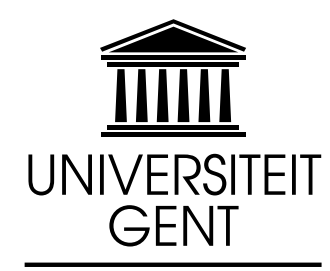





\section{Strangeness Production \\ ON THE NUCLEON}

\section{STIJN JANSSEN}

Promotor: Prof. dr. JAN RYCKeBuSCH

Proefschrift ingediend tot het behalen van de graad van

Doctor in de Wetenschappen: Natuurkunde

Universiteit Gent

Faculteit Wetenschappen

Vakgroep Subatomaire en Stralingsfysica

Academiejaar 2001-2002

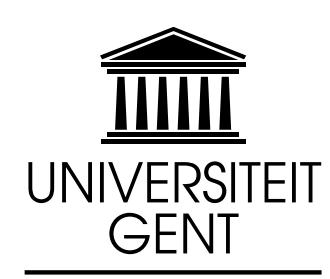


Cover: Composition $V$

Wassily Kandinsky (1866-1944) 
There is no excellent beauty that hath not some strangeness in the proportion - Francis Bacon, quoted by Murray Gell-Mann 



\section{Vooraf}

Bij het voordragen van dit werk wil ik allereerst mijn promotor, Jan Ryckebusch, van harte bedanken. Niet alleen voor het grondig nalezen van deze tekst, maar ook voor de vele stimulerende discussies die uiteindelijk tot dit werk hebben geleid. Daarnaast wil ik Wim, Dimitri en Tim bedanken voor de fijne samenwerking in onze groep en de kritische benadering van mijn onderzoek. Marc Vanderhaeghen wil ik bedanken voor de verhelderende discussies over Regge theorie. Een woord van dank gaat ook naar mijn ouders voor de steun en blijvende interesse in mijn werk. Voor Annik, mijn "vrouwke", zijn de strange quarks ondertussen een beetje minder vreemd geworden en ze heeft (moeten) leren leven met het feit dat mijn gedachten soms in "fysica-land" zijn. Bedankt voor je steun.

\section{Stijn Janssen,}

mei 2002 


\section{Contents}

Vooraf $i$

Table of Contents $\quad$ iii

1 Introduction 1

2 Isobar Model for KY Production $\quad 9$

2.1 Lagrangian Formalism . . . . . . . . . . . . . . . . . . . 9

2.2 Gauge Invariance . . . . . . . . . . . . . . . . . . . 12

2.3 Background Contributions . . . . . . . . . . . . . . . . . 14

2.3.1 SU(3)-flavor Symmetry . . . . . . . . . . . . . . . 14

2.3.2 Background Models . . . . . . . . . . . . . . . . . 16

2.4 Reaction Dynamics . . . . . . . . . . . . . . . . . . 22

3 Strangeness Photoproduction 25

3.1 Observables for KY Photoproduction . . . . . . . . . . . . . . . 25

3.2 K^ Photoproduction ... . . . . . . . . . . . . . . . 29

3.2.1 Resonance Contributions . . . . . . . . . . . . . . . . . . . 29

3.2.2 Background Contributions . . . . . . . . . . . . . 31

3.2.3 Hadronic Form Factors . . . . . . . . . . . . . . . . . . 39

3.2.4 Missing Resonances . . . . . . . . . . . . . . . . . . 43

$3.3 \mathrm{~K} \Sigma$ Photoproduction . . . . . . . . . . . . . . . . 44

3.3.1 Resonance Contributions . . . . . . . . . . . . . . . . 45

3.3.2 Background Contributions . . . . . . . . . . . . . . 48

4 Strangeness Electroproduction $\quad 61$

4.1 Observables for KY Electroproduction . . . . . . . . . . . . . . . . 62

4.2 Gauge Invariance . . . . . . . . . . . . . . . . . . . 65

4.3 Results and Discussion . . . . . . . . . . . . . . . . . 67

4.3 .1 K^ Electroproduction . . . . . . . . . . . . 67 


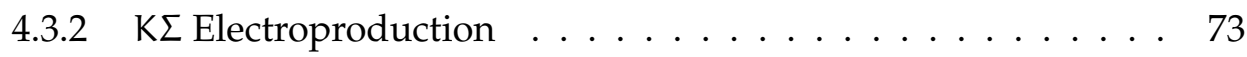

5 Regge Model for KY Production $\quad 77$

5.1 Regge Theory . . . . . . . . . . . . . . . 78

5.2 KY Photo and Electroproduction . . . . . . . . . . . . . . . . . . . . . . . . . . .

5.3 Extensions of the Regge Model . . . . . . . . . . . . . . . . . . 90

5.3 .1 Duality Corrections . . . . . . . . . . . . . . . . . 90

5.3 .2 Beyond the Regge Limit . . . . . . . . . . . . . . . . . . . 99

6 Conclusion and Outlook $\quad 109$

A Effective Field Theory and Pseudo-scalar Meson Production 115

A.1 Effective Lagrangians for $\mathrm{p}\left(\gamma, \mathrm{K}^{+}\right) \wedge$ Reactions . . . . . . . . . . . . . . . . . . . . . . . 115

A.1.1 Interaction Lagrangians . . . . . . . . . . . . . 115

A.1.2 Propagators . . . . . . . . . . . . . . . . 118

A.2 Effective Lagrangians for $p(\gamma, \mathrm{K}) \mathrm{Y}$ Reactions . . . . . . . . . . . 119

A.2.1 Born Terms . . . . . . . . . . . . . . . . . . . . . . 119

A.2.2 Isospin Symmetry and Coupling Constants . . . . . . . . 121

A.2.3 Numerical Values . . . . . . . . . . . . . . . . . . . . . . . . 124

A.3 Electromagnetic Form Factors for $\mathrm{p}\left(\mathrm{e}, \mathrm{e}^{\prime} \mathrm{K}\right) \mathrm{Y}$ Reactions . . . . . . . . 124

A.3.1 Baryonic Form Factors . . . . . . . . . . . . . . . . . 124

A.3.2 Mesonic Form Factors . . . . . . . . . . . . . . 130

$\begin{array}{ll}\text { B Minimization Procedure } & 133\end{array}$

$\begin{array}{lrr}\text { C Regge Scattering Amplitude } & 139\end{array}$

$\begin{array}{ll}\text { D The Veneziano Model } & 151\end{array}$

$\begin{array}{ll}\text { Bibliography } & 155\end{array}$

$\begin{array}{lr}\text { Nederlandse Samenvatting } & 161\end{array}$ 


\section{Chapter 1}

\section{Introduction}

One of the most conspicuous properties of nature is the great diversity of size or length scales in the structures of the world [1]. An ocean, for example, has currents that persist for thousands of kilometers. It also has waves of less than a centimeter to several meters. At far more finer resolution, sea water must be regarded as an aggregate of molecules whose characteristic scale of length is roughly $10^{-10} \mathrm{~m}$. Thereby, every scale exhibits particular properties and phenomena which are described by totally different physical concepts.

This example illustrates a fundamental issue in all branches of physics, namely, to find the appropriate degrees-of-freedom. It is obvious that a fully microscopic model of a physical structure can contain the correct constituents and dynamics but, frequently, it will fail in describing phenomena extending over a larger scale. Therefore, it is often much more efficient to identify some global, "nonfundamental" constituents on this larger scale. Of course, it is of crucial importance to understand the connections between the various scales and the appropriate degrees-of-freedom at every level. Only then, the physical problem can be identified as fully understood.

In the area of nuclear and subatomic physics, one is confronted with a similar situation as the one sketched above, in the sense that the fundamental constituents of matter do not represent themselves as the proper degrees-of-freedom to describe the richness of phenomena which one is facing in the subatomic world.

In the sixties, one realized that the hundreds of hadrons that had been identified experimentally, did not represent the fundamental constituents of matter. As it 
turned out, hadrons contained an internal structure and could be classified on the basis of a restricted set of new particles at a lower level. These findings marked the start of the development of a totally new description of the constituents of matter and the strong interaction in terms of quark and gluon degrees-of-freedom. Since then, the fundamental equations for the description of the dynamics of quarks and gluons have been summarized in a concise theory which is known as Quantum Chromo Dynamics (QCD).

In hadronic physics, the length scale is mostly expressed as an energy scale. For very high energies in the multi-GeV regime, corresponding to circumstances which can only be reached in a few highly specialized facilities in the world, the QCD Lagrangian can be solved perturbatively and describes the behavior of the (asymptotically free) quarks and gluons perfectly. On the other hand, at lower energies, which corresponds to the world we live in, the strong coupling constant becomes too large for ordinary perturbative techniques to be applicable and it becomes a far from trivial task to solve the basic equations of QCD theory. The increasing strong coupling constant by decreasing energies is also responsible for the fact that, at this level, individual quarks and gluons cannot be revealed anymore. There are strong indications that only well defined conglomerates of quarks and gluons can survive in nature. This observation is coined as the "confinement principle". It explains why nature presents itself in terms of hadrons like protons, neutrons and pions although they are not the basic constituents of matter.

Summarizing, it is well established that QCD is at the basis of the composite structure of the hadrons, but it turns out that in many cases QCD does not provide the appropriate degrees-of-freedom to describe the properties of hadronic matter at a larger scale. For example, the concept of structureless hadrons turns out to be much more efficient in describing the properties of nuclei. At intermediate energies, though, some of the properties of the hadrons are surprisingly well explained in terms of constituent quarks without taking explicitly the gluons into account.

The idea of multiple scales in nuclear physics is made clear in Fig. 1.1. At low energies in the $\mathrm{MeV}$ regime, hadrons can be treated as structureless particles. At medium energies, part of the substructure of the hadrons is revealed and the concept of constituent quarks turns out to be effective for understanding their major properties (like magnetic moments e.g.). Upon further decreasing of the length 

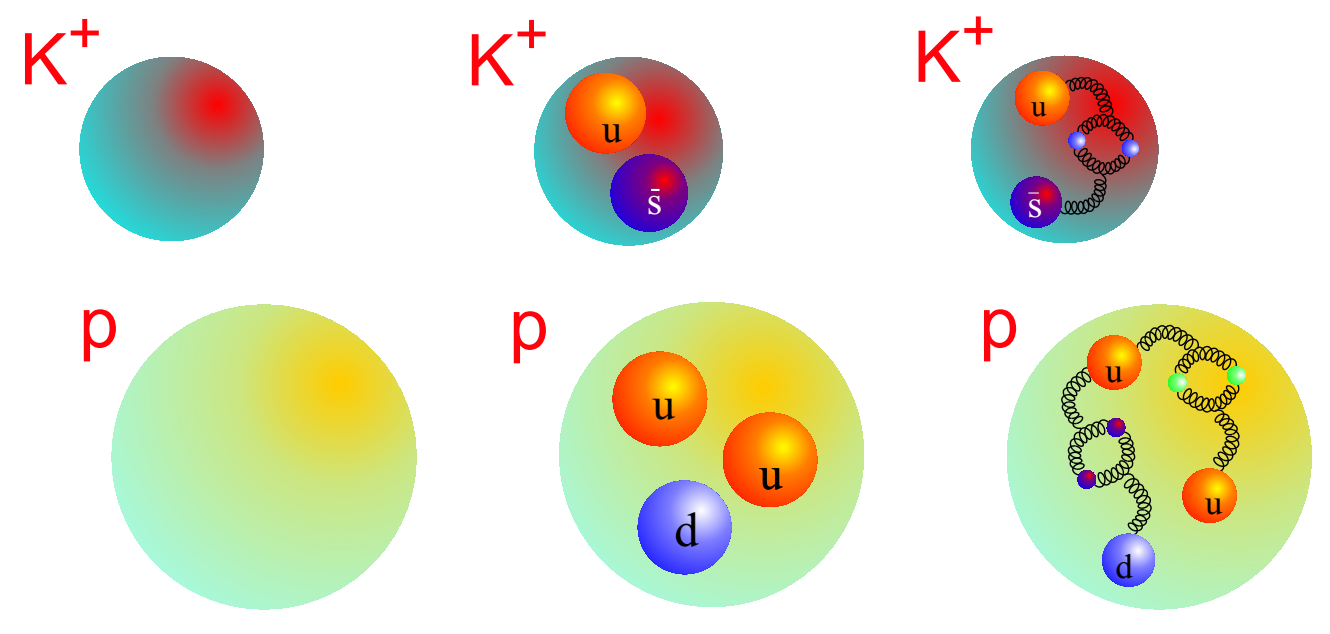

\section{Energy}

Figure 1.1 A proton and a kaon at different energy (or length) scales. At low energy, the hadrons are structureless. By increasing energy, the constituent quarks are revealed and at high energies, the full QCD dynamics in terms of quarks and gluons is observed.

scale, the full QCD dynamics and the partonic nature of hadronic matter becomes accessible.

It is an intellectual challenge for humanity, though, to fully understand the mechanisms that govern the physics of subatomic matter as it presents itself in our every-day world. Thereby, it is essential to bridge the gap between nuclear physics, where hadrons are the basic degrees-of-freedom and the QCD theory of quarks and gluons. At present, it is far from being fully understood what kind of mechanisms make quarks and gluons to create the hadrons as they are observed. What is the nature and the driving mechanisms of the "phase transition" between those different scales?

It speaks for itself that to gain deeper insight into the structure of the nucleon it is essential to fully understand its excited states. After all, the excitation spectrum of the nucleon reflects its underlying structure and must contain in some way a signature stemming from the constituents at a lower level. Information about the nucleon resonances can be gathered by a rich variety of experiments. Thereby, a 
general principle is to transfer an amount of energy to the nucleon by means of a hadronic or electromagnetic probe. Due to this energy-momentum transfer, the nucleonic system is left into one of its excited states which eventually decays into a set of final particles. In the plethora of experiments, the ones which employ an electromagnetic probe take advantage of the fact that to date the electromagnetic coupling to a nucleon is better understood than its hadronic counterpart. At present, highduty electron and photon facilities like CEBAF, ELSA, MAMI, SPring-8, GRAAL, LEGS, MIT-Bates... provide data for electromagnetically induced reactions on the nucleon with unprecedented accuracy. One of the major challenges for the field is extracting from those data reliable information about the properties of resonances, in an as model independent fashion as possible.

Over the last few decades, a substantial amount of information concerning the spectrum of the nucleon has been gathered. Most of this knowledge has been extracted from pion induced and pion production reactions. Since long, however, it has been realized that pionic reactions may be too restrictive with regard to the specific type of intermediate resonant states which can be excited. This assumption is supported by recent (constituent) quark model calculations [2-5] predicting far more excited states than observed in pion production experiments. This observation is known as the "missing resonance" phenomenon in medium energy physics. A fundamental question which awaits further investigations is whether it is possible to observe those "missing resonances" in decay channels which do not involve a pion or whether there is something seriously wrong with the present (constituent) quark models as a basis for our understanding of the structure of hadrons.

Reactions, which have internationally received a lot of attention in recent years, are photoproduction reactions that involve "open" and "closed" strangeness in the final channel. It is believed that the occurrence of a strange s $\bar{s}$ quark pair in the reaction dynamics, in addition to the presence of up and down quarks, can shed a new light on our understanding of the spectrum and the underlying dynamics of hadrons. Moreover, quark models predict appreciable decay of some of the missing resonances into the strange channels [4]. All this makes strangeness production a very promising field. One of the reactions involving strangeness in the final channel is the electromagnetic production of kaons, in brief denoted as $p(\gamma, K) Y$ with $Y=\Lambda, \Sigma$. The theoretical modeling of photon and electron induced 
open-strangeness production in the resonance region (i.e. photon lab energies from threshold up to $2 \mathrm{GeV}$ ) constitutes the subject of this thesis.

The modeling of kaon photoproduction processes started back in the sixties by the pioneering work of Kuo [6] and Thom [7]. This work marked the start of a long series of theoretical efforts in this field [8-16]. The earliest works clearly lacked the presence of a reliable database. In those days, pioneering kaon photoproduction experiments were performed at Bonn, Tokyo, Cornell and CalTech [17-22] but the experimental facilities did only cover a limited energy range above the kaon production threshold. To make matters even worse, the accuracy of the measurements suffered from the very low counting rates, reflecting cross sections of the order of microbarns, compared to for example $\pi$ photoproduction which are characterized by cross sections in the milibarn range. One had to wait until 1998, when the SAPHIR collaboration at Bonn $[23,24]$ released the first extensive and accurate database for all three $\gamma p \rightarrow \mathrm{K}^{+} \Lambda, \mathrm{K}^{+} \Sigma^{0}$ and $\mathrm{K}^{0} \Sigma^{+}$reactions. The results of this experiment clearly triggered renewed interest at various theoretical groups [25-32].

In general, the theoretical description of meson photoproduction goes along two major paths. On one hand, there are the parton based models. Thereby, a (constituent) quark model is at the basis of calculations of the reaction dynamics [33-35]. The partonic constituents can also be taken into account along the lines proposed by Regge theory [16] which is a high energy theory or in chiral models which are a low energy approximation of the QCD formalism [36,37]. The problem can also be tackled starting from purely hadronic degrees-of-freedom. In such an approach, the hadrons are treated as effective particles with specific properties [13,27]. From this dual approach, it is hoped that one can reach a deeper level of understanding of the "phase transition" between the low and high energy description of subatomic matter.

This work falls apart in two major parts which closely follow this twofold way. In Chaps. 2-4, the starting point for modeling $p(\gamma, K) Y$ and $p\left(e, e^{\prime} K\right) Y$ reactions is a description in terms of hadronic degrees-of-freedom. This type of description is commonly referred to as the "isobar model". Despite the long history and the large amount of both experimental and theoretical efforts, a complete understanding of the $p(\gamma, K) Y$ reaction mechanisms in such a model remains elusive. One of the major reasons for this rather limited knowledge is that the reaction is fully located 
in the so called third resonance region. From a kinematic point of view, there are more than twenty resonances as likely candidates to participate in the reaction. A number of those resonances is only poorly characterized. Thereby, it is believed that they all have large widths of hundreds of $\mathrm{MeV}$, resulting in a broad energy smearing of every state. This complicates the theoretical description since various resonances overlap in the same energy region, resulting in an erratic interference pattern. In that respect, in processes like $p(\gamma, \pi) N$ or $p(\gamma, \eta) p$ near threshold, one does not face similar difficulties since their reaction dynamics is dominated by a single (isolated) resonance.

In this work we have tried to identify the most important resonance contributions in the $p(\gamma, \mathrm{K}) \mathrm{Y}$ reaction dynamics. Thereby, special attention is paid to the issue of the missing resonances and there is looked for signals of such states. By determining the various resonance contributions, one can extract values for the coupling constants. These quantities, which express the strength of each resonance in the process, can be compared to quark model predictions.

Apart form a limited knowledge with respect to the various resonance contributions, also the description of the non-resonant, so called background, diagrams poses a serious challenge to models which aim at describing $\mathrm{p}(\gamma, \mathrm{K}) \mathrm{Y}$ and $p\left(e, e^{\prime} K\right) Y$ processes. It turns out that some model dependence in determining these background contributions cannot be avoided. In this work, a profound study of the issue of the background terms is performed. Various possibilities to parameterize this part are presented and consequences for model predictions and extracted parameters are explored.

In addition to an isobar description, Chap. 5 collects the results of a Regge theory based model for $p(\gamma, K) Y$ and $p\left(e, e^{\prime} K\right) Y$ processes. Regge theory has a rich history going back to the late fifties and has proven to be a successful and efficient approach for understanding a variety of high energy reactions involving hadronic and electromagnetic probes. The basic idea here, is not to start from individual hadronic particles as key players in the reaction dynamics, but, rather start from classes of particles, called trajectories, with some general properties determined by partonic degrees-of-freedom. In that respect, Regge theory can constitute a direct bridge between the hadronic and partonic picture of certain processes. The presented Regge model is known to provide an excellent description of the high 
energy $p(\gamma, \mathrm{K}) \mathrm{Y}$ data at forward angles $[16,38,39]$. In this work, we will mainly concentrate on possible extensions of Regge based models into the resonance region.

For the sake of improving the general readability of this work, the more technical aspects with regard to the isobar and Regge model, have been collected in a series of appendixes. 



\section{Chapter 2}

\section{Isobar Model for KY Production}

In this chapter, we give an outline of the isobar model that will be used to describe and interpret the strangeness photo- and electroproduction processes. In Sec. 2.1, we concentrate on the Lagrangian formalism which is at the basis of the isobar model. The issue of gauge invariance is addressed in Sec. 2.2. An important part of this work is dedicated to the treatment of the background contributions in the process. We sketch the main ideas of this issue in Sec. 2.3. Finally, in Sec. 2.4 we come to a general description of the reaction dynamics in the isobar model.

\subsection{Lagrangian Formalism}

In an isobar model, the physical degrees-of-freedom are the hadrons and their excited states. In such a framework, meson photoproduction reactions are modeled with the aid of effective Lagrangians. Every (intermediate) particle in the reaction dynamics is treated as an effective field with its own characteristics like mass, photocoupling amplitudes and strong decay widths. To calculate the reaction amplitude for a given process, the dominant Feynman diagrams have to be identified. In an initial step, only the first order terms are taken into account. Those terms, referred to as the "tree level", only contain diagrams as collected in Fig. 2.1 and do not introduce additional loops. Obviously, this approximation can only be a first step towards developing a more complete description of the reaction. An important drawback is that final state interaction effects, generated through the rescattering of the final state particles, are not taken into account in those first order terms. 

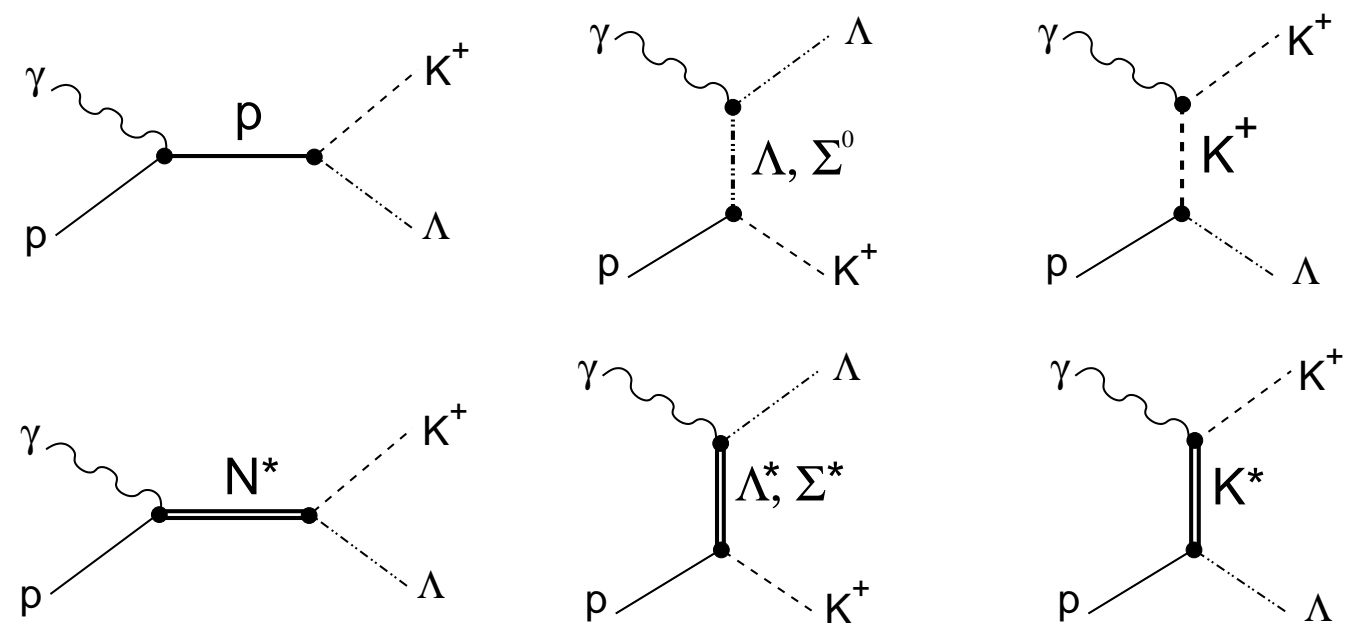

Figure 2.1 Diagrams contributing to the $p\left(\gamma, \mathrm{K}^{+}\right) \wedge$ process at tree level. The upper row corresponds to the Born terms in which a proton is exchanged in the s-channel, a $\Lambda$ or $\Sigma^{0}$ in the $\mathrm{u}$-channel and a $\mathrm{K}^{+}$in the $\mathrm{t}$-channel. The lower row shows the corresponding diagrams with the exchange of an excited particle or resonance. Analogous diagrams exist for the other isospin channels.

Rescattering contributions such as $\gamma p \rightarrow \pi N^{*} \rightarrow K Y$ are of second order and can for example be handled in a full coupled-channel analysis. Recently, Chiang et al. estimated the effects of coupled-channel mechanisms on the $p\left(\gamma, \mathrm{K}^{+}\right) \wedge$ cross sections of the order of $20 \%$ [28]. Admittedly, this is a substantial effect. However, apart from the uncertainties inherent to coupled-channel approaches, such as unknown phase shifts and off-shell rescattering ambiguities, these results indicate that tree level diagrams constitute the major part of the $p\left(\gamma, \mathrm{K}^{+}\right) \wedge$ reaction dynamics. In this work, we will show that even in a tree level description of $p(\gamma, K) Y$ processes, a reliable extraction of resonance parameters is far from evident and subject to uncertainties. Therefore, we believe that, in addition to directing efforts towards dealing with the coupled-channel final state interaction effects, a proper treatment and understanding of the first order tree level terms is mandatory.

In this light, we have restricted ourselves to a tree level description of the reaction and only diagrams of the type as shown in Fig. 2.1 (for the case of the $p\left(\gamma, \mathrm{K}^{+}\right) \wedge$ reaction) are taken into account. To evaluate these diagrams, one can rely on an effective field theory which determines the structure of the propagators and the vertices. The major ingredients of this theory are outlined in detail in 
App. A. For the propagators of the spin- $1 / 2$ baryons, pseudo-scalar mesons and (axial) vector mesons, the standard expressions are used. For the spin-3/2 particles, the Rarita-Schwinger form for the propagator is adopted. For the interaction Lagrangians, describing the coupling of the particles, there is some ambiguity with respect to the structure of the KYN vertex in the sense that one can make use of either pseudo-scalar or pseudo-vector coupling (or, a combination of both). For the kaon photoproduction case, this issue has been studied by several authors [26, 40,41], but neither of the two possible schemes has as yet been identified as favorable. All results reported in this work are obtained with the pseudo-scalar option.

The interaction Lagrangians depend on an effective coupling, determining the strength of the corresponding underlying interaction. In an effective field theory, the coupling constants for each of the individual resonances are not determined by the theory itself. They are treated as free parameters and must be extracted by performing a global fit of the model calculations to the available data base. In a second step, these values can be compared to quark model predictions $[2,4,5$, 42], although the effects of final state interactions, which are expected to be partly absorbed in the effective couplings, may somehow obscure the results. Throughout this work, we will compare our model predictions to the SAPHIR data base $[23,24]$ to determine the vertex couplings. When performing a global fit to $\mathrm{N}$ data points, the optimum set of coupling constants is the one that produces the lowest value for $\chi^{2}$, which is defined in the standard manner:

$$
\chi^{2}=\frac{1}{N} \sum_{i}^{N} \frac{\left[X_{i}-Y_{i}\left(a_{1}, \ldots a_{n}\right)\right]^{2}}{\sigma_{X_{i}}^{2}} .
$$

Here, $X_{i}$ are the measured observables, $\sigma_{X_{i}}^{2}$ the corresponding standard deviations and $Y_{i}\left(a_{1}, \ldots a_{n}\right)$ the theoretical prediction for the variables $X_{i}$. The $a_{j}$ 's denote the parameters of the theory, being coupling constants and cutoff masses. Apart from the two Born term coupling constants $g_{K^{+}} \wedge p$ and $g_{K^{+} \Sigma^{0} p^{\prime}}$ all the extracted resonance $(R)$ parameters $G_{R}$ are a combination of a photocoupling (sometimes called a magnetic transition moment) and a strong hadronic coupling. A description of the various types of resonance parameters $G_{R}$, their normalization and their connection to the Lagrangian structure is given in App. A. The technical details of the $\chi^{2}$ minimization procedure and some related numerical problems are postponed to App. B. 
The effective Lagrangians of the hadronic vertices describe point-like interactions. To account for the finite extension of the hadrons at the strong vertices, it is a common procedure to introduce phenomenological form factors [43]. In this work, these form factors take on a dipole form:

$$
F_{x}(\Lambda)=\frac{\Lambda^{4}}{\Lambda^{4}+\left(x-m_{x}^{2}\right)^{2}} \quad(x=s, t, u)
$$

where $x$ represents the off-shell momentum squared at the vertex. $\Lambda$ is the cutoff value which can be interpreted as a short-distance scale of the effective theory. We note that there is some arbitrariness in the functional form of the form factor. In order to minimize the number of free parameters, we adopt one cutoff mass $\Lambda$ for all the Born diagrams (upper row of Fig. 2.1) and one global cutoff parameters for all the resonance exchange terms (lower row of Fig. 2.1).

\subsection{Gauge Invariance}

A major implication of introducing hadronic form factors is that gauge invariance of the amplitude is broken at the level of the Born terms. Gauge invariance corresponds to charge conservation and consequently represents a fundamental symmetry of any theory dealing with electromagnetic interactions. This reflects itself in a strict relation between the amplitudes which involve an electric coupling. For the Born terms and the $\mathrm{p}\left(\gamma, \mathrm{K}^{+}\right) \wedge$ case, those amplitudes with an electric coupling part read:

$$
\begin{aligned}
\varepsilon_{\mu} M_{s-\text { electric }}^{\mu} & =\varepsilon_{\mu} e g_{K^{+} \wedge p} \bar{u}_{\Lambda} \gamma_{5} \frac{\not p+\not k+m_{p}}{s-m_{p}^{2}} \gamma^{\mu} u_{p} \\
\varepsilon_{\mu} M_{t}^{\mu} & =\varepsilon_{\mu} e g_{K^{+} \wedge p} \bar{u}_{\Lambda} \frac{2 p_{K}^{\mu}-k^{\mu}}{t-m_{K}^{2}} \gamma_{5} u_{p} .
\end{aligned}
$$

Here, $p^{\mu}, k^{\mu}$ and $p_{K}^{\mu}$ are the four momenta of the proton, photon and kaon, respectively. Further, $\varepsilon_{\mu}$ is the polarization vector of the photon and $u_{p}$ and $u_{\wedge}$ are the spinors of the proton and $\wedge$-hyperon. The Lorentz invariant Mandelstam variables are defined as:

$$
s=(p+k)^{2}, \quad t=\left(p_{K}-k\right)^{2}, \quad u=\left(p_{\Lambda}-k\right)^{2}
$$


Note that for the other isospin channels similar electric coupling amplitudes exist as those given in Eq. (2.3), although they can appear in other Born amplitudes. In the $\mathrm{n}\left(\gamma, \mathrm{K}^{+}\right) \Sigma^{-}$case, for example, the two amplitudes which contain an electric coupling emerge in the $t$ - and $u$-channel.

To be gauge invariant, the total amplitude has to fulfill the Lorentz condition:

$$
k_{\mu} M^{\mu}=0
$$

Using the Dirac equation, it is easily proven that for on-shell external particles the combination of both amplitudes in (2.3) vanishes in the contraction with $k_{\mu}$. However, this will no longer be the case if the amplitudes of Eqs. (2.3a) and (2.3b) are modified by $F_{s}(\Lambda)$ and $F_{t}(\Lambda)$ as defined in Eq. (2.2), respectively. Note that the (axial) vector meson and resonance exchange terms, which are characterized by the electromagnetic interaction Lagrangians of the type (A.6), (A.7), (A.11) and (A.14), are gauge invariant by construction. Consequently, the gauge invariance of those terms is not effected through the introduction of form factors.

As suggested by Haberzettl, the gauge invariance of the Born terms can be restored by adding extra contact terms [44]. Those contact terms, which introduce a new form factor $\widehat{F}$, are determined in such a manner that the gauge violating terms are exactly canceled. For the $\mathrm{p}\left(\gamma, \mathrm{K}^{+}\right) \Lambda$ case these terms read:

$$
\begin{aligned}
\varepsilon_{\mu} M_{\text {contact }}^{\mu}= & \varepsilon_{\mu} e g_{K+\Lambda p} \bar{u}_{\Lambda} \gamma_{5}\left[\frac{2 p^{\mu}+\not k \gamma^{\mu}}{s-m_{p}^{2}}\left(\widehat{F}-F_{s}\right)\right. \\
& \left.+\frac{2 p_{K}^{\mu}}{t-m_{K}^{2}}\left(\widehat{F}-F_{t}\right)\right] u_{p} .
\end{aligned}
$$

This contact term indeed cancels the gauge violating terms and eventually results in a modification of all electric terms with the same form factor $\widehat{F}$. With regard to the functional form of the additional form factor $\widehat{F}$, Haberzettl argued that this form factor is acting on terms of different channels (on s- and t-channel terms, in the $p\left(\gamma, K^{+}\right) \wedge$ case $)$ and therefore, he proposed a linear combination of the form [45]:

$$
\widehat{F}=\widehat{F}_{H} \equiv a_{s} F_{s}(\Lambda)+a_{t} F_{t}(\Lambda)+a_{u} F_{u}(\Lambda)
$$

The $a_{x}$ coefficients have to satisfy the relation $a_{s}+a_{t}+a_{\mathfrak{u}}=1$, in order to produce the correct infrared or soft photon limit $(k \rightarrow 0)$. 
Recently, Davidson and Workman criticized the functional form for $\widehat{F}$ of Eq. (2.7) [46]. They argued that after introducing a form factor $\widehat{F}_{H}$ of the type proposed, the contact term of Eq. (2.6) contains poles and, although these poles are outside the physical region of the process, the term cannot be considered as a contact term. Indeed to be a contact term, $\widehat{F}$ has to fulfill the conditions:

$$
\begin{array}{ll}
\widehat{F}=F_{s} & \text { if: } s=m_{p}^{2}, \\
\widehat{F}=F_{t} & \text { if: } t=m_{K}^{2},
\end{array}
$$

to counterbalance the poles. It is obvious that with the prescription of Eq. (2.7), both conditions can not be simultaneously met, except in the soft photon limit. An alternative recipe, that fulfills the conditions (2.8) by construction, was suggested by Davidson and Workman and reads [46]:

$$
\widehat{F}=\widehat{F}_{D W} \equiv F_{s}(\Lambda)+F_{t}(\Lambda)-F_{s}(\Lambda) F_{t}(\Lambda)
$$

Unless specified otherwise, in the forthcoming numerical calculations we have adopted this gauge restoration procedure by Davidson and Workman.

\subsection{Background Contributions}

\subsubsection{SU(3)-flavor Symmetry}

When constructing qqq and $\mathrm{q} \overline{\mathrm{q}}$ states, where the q can be either an $u p$, down or strange quark, one is left with $27\left(=3^{3}\right)$ possibilities to obtain a (ground state) baryon and $9\left(=3^{2}\right)$ possibilities to construct a (ground state) meson. Those mixed flavor states are described by the SU(3) symmetry group. According to this flavor symmetry, the two sets that represent the baryons and the mesons, can be decomposed in irreducible multiplets. For the 27 states in the baryon set, the reduction becomes: $[10] \oplus[8]_{\mathrm{D}} \oplus[8]_{\mathrm{F}} \oplus[1]$. Here, a distinction is made between the symmetric (D) and anti-symmetric (F) octet. For the 9 mesons, the reduction goes as: $[8] \oplus[1]$. The octet and singlet of the low lying pseudo-scalar mesons and the octet of the physical spin$1 / 2$ baryons are schematically depicted in Fig. 2.2. The relevant quantum numbers that organize these multiplets are the isospin projection $\mathrm{I}_{3}$ and the strangeness quantum number $S$. Alternatively, the $S$ quantum number can be replaced by the hyper charge $\mathcal{Y}$, which is defined as $\mathcal{Y}=S+B$, with $B$ the baryon number. 

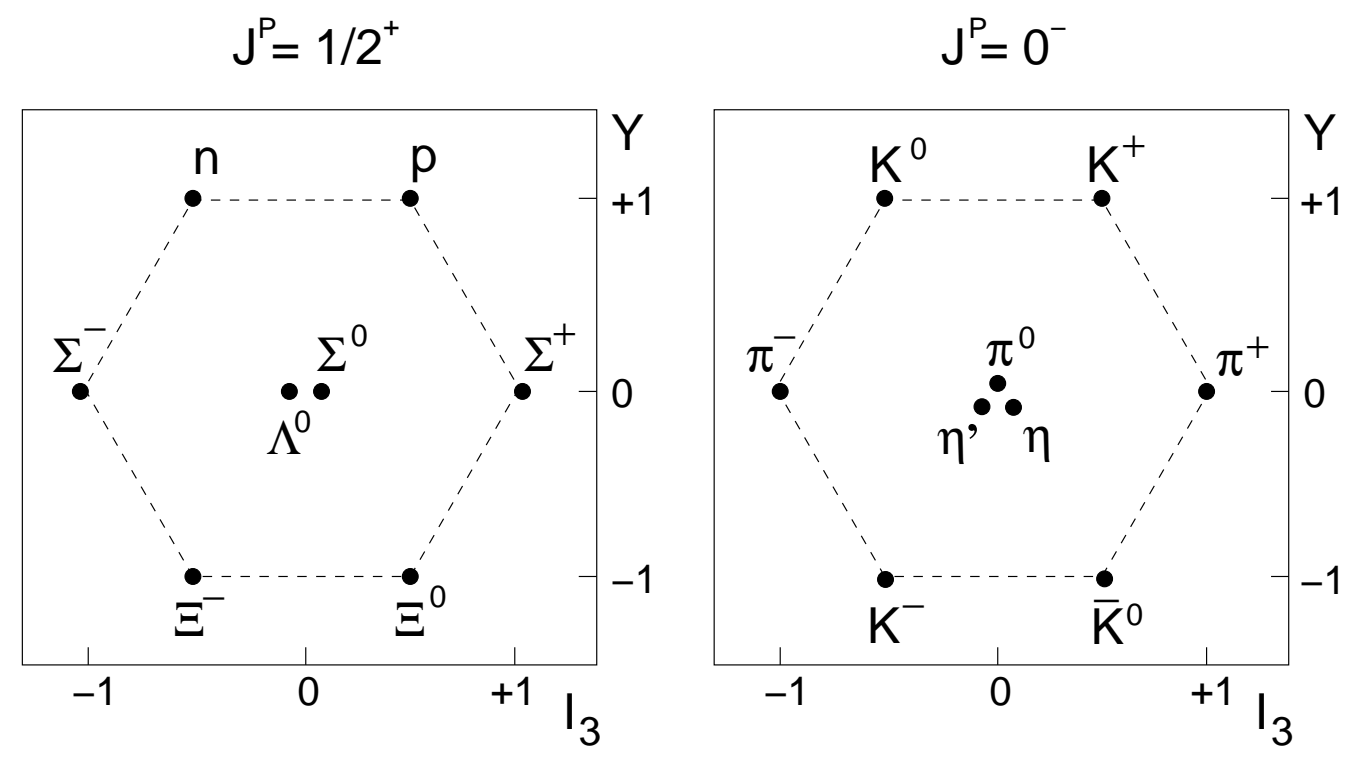

Figure 2.2 The spin-1/2 baryon octet and the pseudo-scalar nonet as organized by SU(3)flavor symmetry. The particles are depicted according to their isospin projection $\mathrm{I}_{3}$ and hyper charge $\mathcal{Y}$.

The SU(3) symmetry, governing the baryon and meson multiplets, can be exploited to establish relations between the coupling constants that connect the particles of the different sets. As such, these SU(3) relations will permit to connect the coupling constants of the up-down $(\pi \mathrm{N})$ sector, which are mostly very well determined, to the coupling constants of the strange sector. Following de Swart, who made an extensive study of this topic in Ref. [47], one can derive the relations:

$$
\begin{aligned}
& g_{K \wedge N}=-\frac{1}{\sqrt{3}}\left(3-2 \alpha_{D}\right) g_{\pi N N}, \\
& g_{K \Sigma N}=\left(2 \alpha_{D}-1\right) g_{\pi N N} .
\end{aligned}
$$

Here, $\alpha_{D}$ is the fraction of symmetric coupling in the $\pi N N$ vertex. To define this quantity, is important to note that the mathematical decomposition of the 27 baryon states into the irreducible multiplets, does not completely correspond with the physically observed particles. For example, the physical $\mathrm{J}^{\mathrm{P}}=1 / 2^{+}$baryon octet, given in Fig. 2.2, is conceived as a linear combination of the two octets $[8]_{\mathrm{D}}$ and $[8]_{\mathrm{F}}$. This composite nature of the physical particles is also reflected in the meson baryon couplings. The degree of mixing is expressed by the quantity $\alpha_{D}$ which is determined to be 0.644 [48]. With the experimental knowledge of $g_{\pi N N}^{2} / 4 \pi=14.4$, the coupling 
constants $g_{K^{+} \wedge p}$ and $g_{K^{+} \Sigma^{0} p}$ are in principle nailed down through Eq. (2.10).

Since the substantial mass difference between the mass of the proton $\left(m_{p}=\right.$ $938.3 \mathrm{MeV})$ and the $\Lambda\left(m_{\Lambda}=1115.7 \mathrm{MeV}\right)$, which essentially originate from interchanging an up into a strange quark, it is well known that the SU(3)-flavor symmetry is broken and the relations (2.10) are not exact. However, it is not unambiguously determined how to define a scale parameter for this symmetry breaking and how to implement this breaking in the coupling constants. Nevertheless, it is commonly assumed that a symmetry breaking of $20 \%$ at the level of the coupling constants is reasonable. With this $20 \%$ deviation from the exact $\mathrm{SU}(3)$ predictions, the following ranges for the $g_{K^{+} \wedge p}$ and $g_{K^{+} \Sigma^{0} p}$ emerge:

$$
\begin{aligned}
-4.5 & \leq \mathrm{g}_{\mathrm{K}^{+} \wedge \mathrm{p}} / \sqrt{4 \pi} \leq-3.0 \\
0.9 & \leq \mathrm{g}_{\mathrm{K}+\Sigma^{0} \mathrm{p}} / \sqrt{4 \pi} \leq 1.3 .
\end{aligned}
$$

\subsubsection{Background Models}

In calculating the contributions from the Born diagrams to the $p(\gamma, \mathrm{K}) \mathrm{Y}$ cross sections, the $g_{K^{+} \wedge p}$ and $g_{K^{+} \Sigma^{0} p}$ coupling constants are the only input parameters. One of the striking observations when dealing with the $\mathrm{p}(\gamma, \mathrm{K}) \mathrm{Y}$ processes in terms of hadronic degrees-of-freedom, is that these Born terms on their own, calculated with values in the ranges of Eq. (2.11), give rise to cross section values which largely overshoot the data. This becomes clear in Fig. 2.3 where the computed total cross sections are plotted in a naive model that only retains point-like (this means before introducing hadronic form factors) Born terms in the reaction process. It is clear that one encounters here a severe problem. Reasonable SU(3) predictions for the coupling constants in the Born terms fail completely when they are compared with the experimental observations. It has to be stressed that Born terms on their own cannot be expected to be responsible for an entire reaction amplitude since they have no imaginary part. On the other hand, the deviation between the calculated strength stemming from the Born terms and the data is so dramatic that it stands beyond doubt that the introduction of mechanisms that can reduce this Born strength to a more realistic level is of primary concern to any model which aims at providing a realistic description of $p(\gamma, K) Y$ processes. Here, we will present four plausible schemes, coined models A, B, C and D, that try to accomplish the goal of properly counterbalancing the Born strength. 

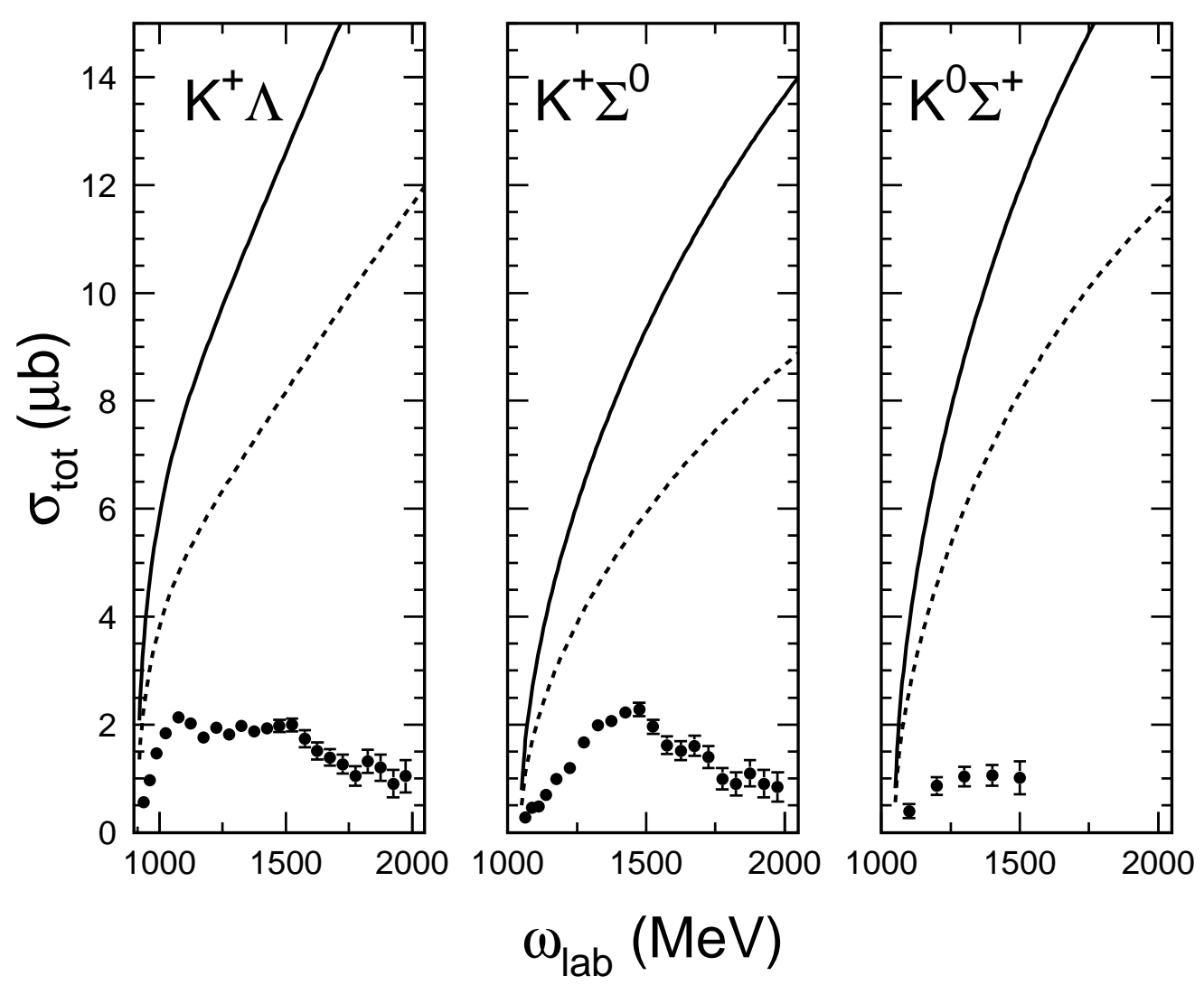

Figure 2.3 The total cross sections for the three strangeness photoproduction processes off the proton as a function of the photon lab energy. The cross sections are obtained in a model which solely includes Born terms in the reaction dynamics and introduces no hadronic form factors. For the solid line, the exact $S U(3)$ predictions for $g_{K+} \wedge_{p}$ and $g_{K+\Sigma} \Sigma_{p}$ are used while for the dashed line, the under limit values of Eq. (2.11) are taken. The data are from Refs. [23,24]. 

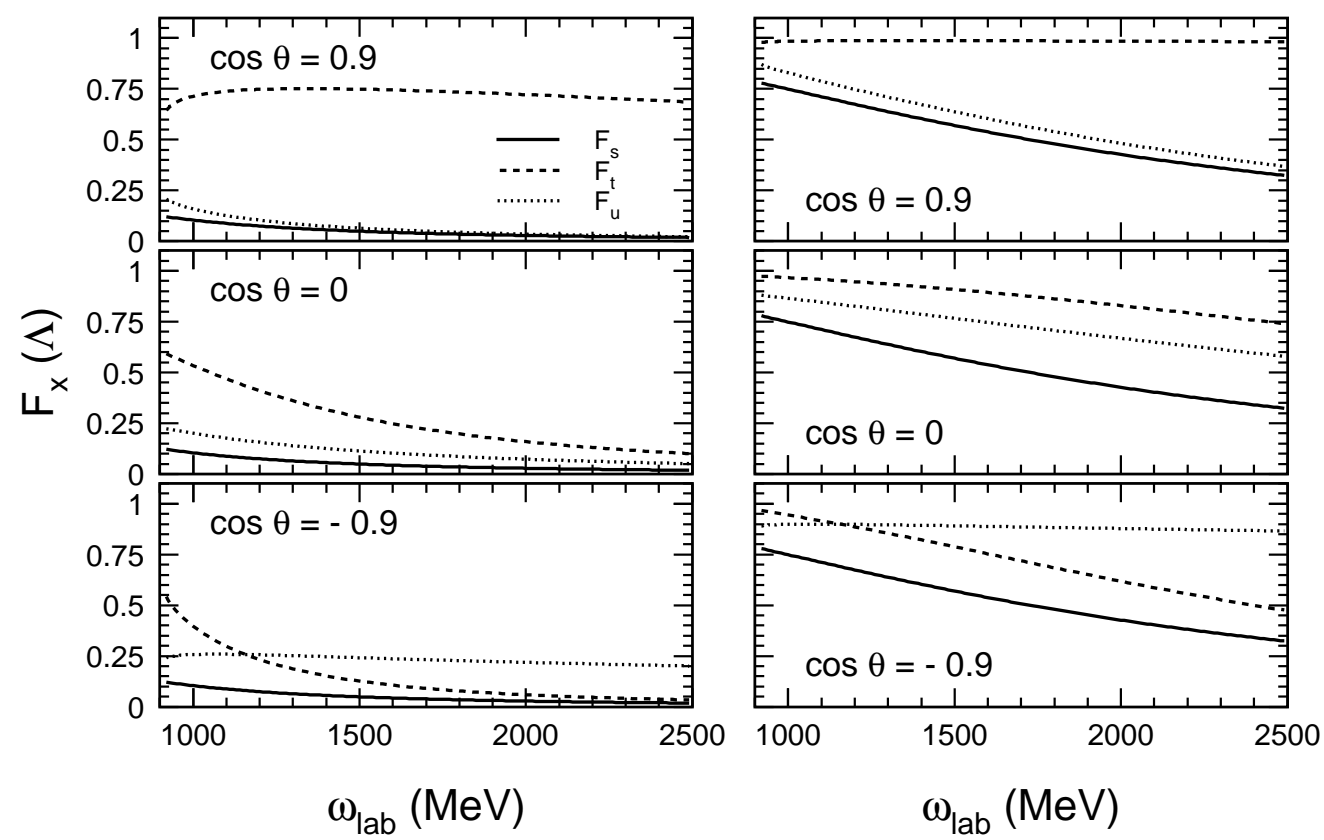

Figure 2.4 The energy dependence of the hadronic form factors for three different kaon center-of-mass angles. The left panels correspond with a cutoff mass $\Lambda=0.8 \mathrm{GeV}$, for the right panels a value of $\Lambda=1.8 \mathrm{GeV}$ is used. The solid, dashed and dotted curves refer to the form factor in the $s-, t$ - and $u$-channel, respectively.

- Model A: The introduction of hadronic form factors in an effective field theory is motivated by the argument that the finite extension of the particles at the hadronic vertex is not taken into account. As such, form factors result in a purely phenomenological masking of the short-range effects of the interaction. The functional form of the form factors is rather arbitrary, but the global effect should always be a smoothing of the high energy behavior of the theory. The energy dependence of the (dipole) hadronic form factors in the three different Born diagrams is given in Fig. 2.4. It is clear that the introduction of hadronic form factors results in a "damping" of bare amplitudes. The scale of reduction depends to a large extent on the adopted value for the cutoff mass. This idea is also made clear in Fig. 2.4. For a soft form factor with a $\Lambda=0.8 \mathrm{GeV}$, the reduction is much more pronounced than for the hard form factor where $\Lambda=1.8 \mathrm{GeV}$. So, it is obvious that hadronic form factors are 
able to reduce the strength stemming from the Born terms. The smaller the cutoff mass $\Lambda$, the larger the reduction of the bare amplitudes will be. However, in order to sufficiently cut the strength from the Born terms without any further modifications of the theoretical framework, the introduction of (unrealistically) small cutoff masses appears necessary. Only when values of the order of the kaon mass are used, the Born strength approaches the order of the observed strength (see Fig. 2.5). It goes without saying that one can pose some serious questions about this procedure. With such small cutoff masses, the form factors start playing a predominant role in the reaction dynamics, not only in the high energy region but even at threshold. At this point, it is worth stressing that in isobar descriptions of $p(\gamma, \pi) N$ or $p(\gamma, \eta) p$ reactions, hadronic form factors do not seem to exhibit such a dominant role $[49,50]$. Despite this, the application of this technique is able to reproduce the available data fairly well, as will be shown in Chap. 3.

- Model B: A second option for counterbalancing the strength from the Born terms is the introduction of hyperon resonances in the $u$-channel. We observe a destructive interference of the $u$-channel hyperon resonance terms with the other background terms. In this way, this procedure is a natural mechanism to reduce the Born strength. In Fig. 2.5, we show the total cross section for the $p\left(\gamma, K^{+}\right) \wedge$ process where we have added two $\Lambda^{*}$ resonances $\left(S_{01}(1800)\right.$ and $P_{01}(1810)$ ) to the Born terms. For those Born terms, we have now used a hard form factor cutoff value. As can be seen in the figure, a reasonable amount of background strength is obtained. It should be remarked that the particular choice of the two $Y^{*}$ resonances is not made on solid physical grounds. We have observed that also other combinations of $\Lambda^{*}$ and $\Sigma^{*}$ resonances are able to reduce the strength by destructive interferences with the other background terms.

- Model C: A third option consists of simply ignoring the ranges for the coupling constants of Eq. (2.11). This inevitably amounts to using coupling constants that are significantly smaller than what is expected on the basis of (broken) $\mathrm{SU}(3)$ symmetry. With values of only $1 / 4$ of the $\mathrm{SU}(3)$ predictions, a reasonable background strength is produced (see Fig. 2.5). It has to be stressed that purely hadronic reactions, such as $p p \rightarrow p K Y$ processes $[51,52]$ or $\mathrm{Kp}$ 


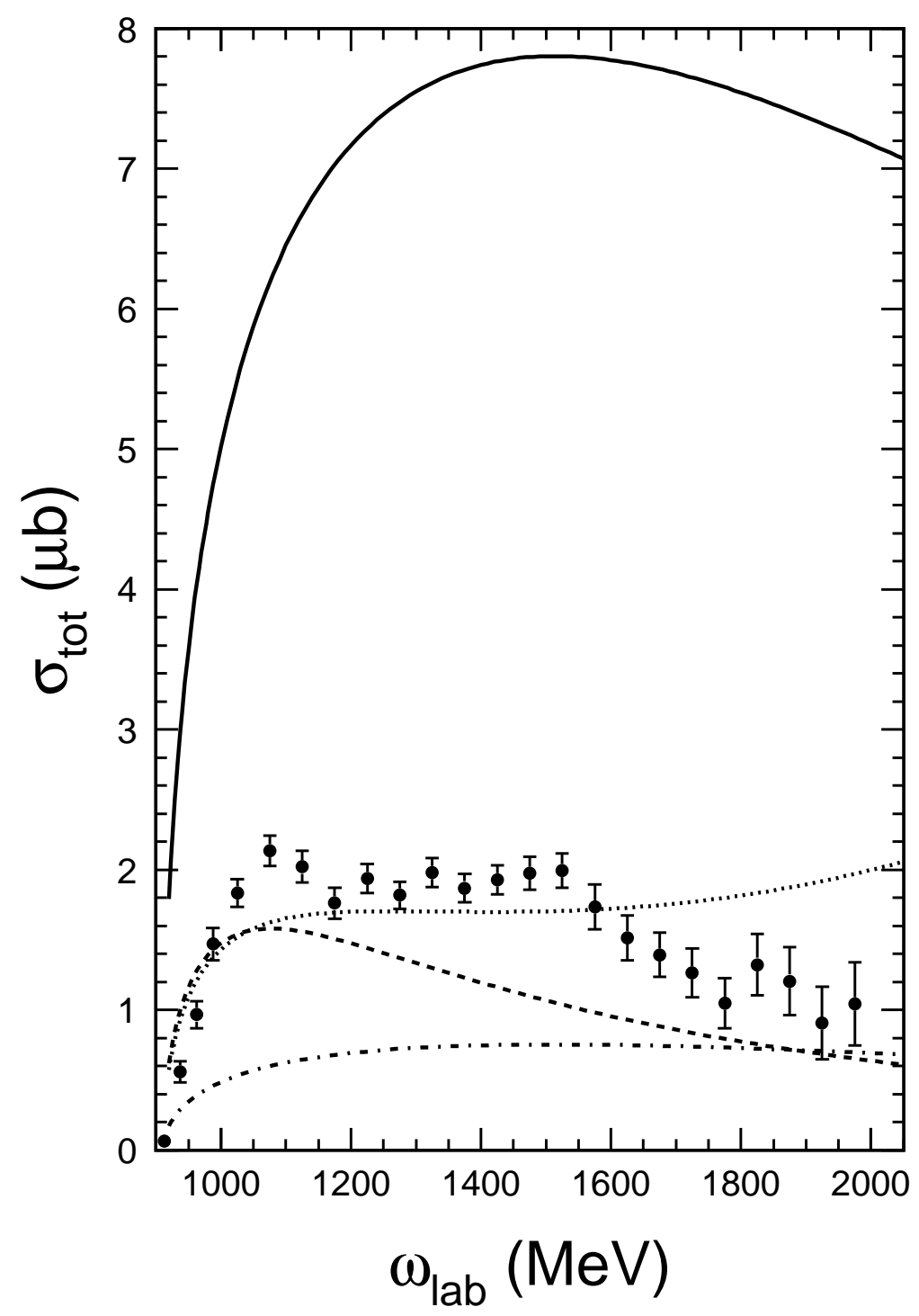

Figure 2.5 Background strengths for the total cross section of the $p\left(\gamma, K^{+}\right) \wedge$ process. The solid line is the strength produced by Born terms with coupling constants in the SU(3) range and modified by a hard form factor with a cutoff mass of $\Lambda=1.6 \mathrm{GeV}$. The dashed line (model A) is obtained from Born terms with soft hadronic form factors $(\Lambda=0.7 \mathrm{GeV})$. The dotted line (model $B$ ) introduces two hyperon resonances $\left(S_{01}(1800)\right.$ and $\left.P_{01}(1810)\right)$ in combination with a hard cutoff $(\Lambda=1.6 \mathrm{GeV})$. For the dot-dashed curve (model $C)$, the Born term coupling constants $g_{K}+\Lambda p$ and $g_{K+\Sigma^{0} p}$ are reduced to a value of $25 \%$ of the SU(3) predictions and a cutoff value of $1.6 \mathrm{GeV}$ is used. The data are from Ref. [23]. 


\begin{tabular}{||c|c|c|c|c||}
\hline \hline Model & SU(3) restrictions & $\wedge$ under limit $(\mathrm{GeV})$ & $\mathrm{Y}^{*}$ in u-channel & NFP \\
\hline A & yes & $\geq 0.4$ & no & 3 \\
B & yes & $\geq 1.5 / 1.6$ & yes & 5 \\
C & no & $\geq 1.1$ & no & 3 \\
D & yes & $\geq 1.1$ & no & 3 \\
\hline \hline
\end{tabular}

Table 2.1 Schematic description of the four models used for treating the Born terms and the "reduction mechanism". The $S U(3)$ restrictions for the $g_{K+} \Lambda_{p}$ and $g_{K+\Sigma^{0} p}$ coupling constants refer to the ranges determined in Eq. (2.11). The $\Lambda$ cutoff masses are those of the hadronic form factors introduced in the Born terms. The table gives the under limit for $\Lambda$ imposed in the fitting procedure. The two values for model $B$ refer to the $K \Lambda$ and the $K \Sigma$ case, respectively. "NFP" refers to the number of free parameters.

scattering [53], can be understood in terms of values for $g_{K^{+}} \wedge p$ and $g_{K^{+} \Sigma^{0} p}$ which are in good agreement with the $\mathrm{SU}(3)$ predictions. Also from a Regge analysis of the high energy $\mathrm{p}(\gamma, \mathrm{K}) \mathrm{Y}$ data [16], values for the coupling constants in agreement with $\mathrm{SU}(3)$ predictions are retrieved. These observations may suggest that there is little room for flavor symmetry breaking beyond the assumptions which led to Eq. (2.11). Despite these reservations, we will retain model $\mathrm{C}$ in the forthcoming discussions. In literature, the procedure of freely varying $g_{K^{+} \wedge p}$ and $g_{K^{+} \Sigma^{0}} p$ parameters is adopted by several authors $[11,12,26]$ and, in general, leads to very satisfactory descriptions of the data.

- Model D: This scheme is an attempt to unite the virtues of the models A and $\mathrm{B}$, at the same time minimizing the number of free parameters which are introduced in the process of computing the background diagrams. In this model, the SU(3)-flavor constraints of Eq. (2.11) are respected during the fitting procedure and no $\mathrm{Y}^{*}$ hyperon resonances are included in the $u$-channel. Moreover, the hadronic cutoff mass $\Lambda$ is allowed to vary freely in the fitting procedure in a range defined by the under limit of $1.1 \mathrm{GeV}$.

Table 2.1 summarizes the basic assumptions for each of the four models to treat the background contributions. To conclude this section, we make some comments 
on model D. This type of background is used in our studies that aimed at determining the $\mathrm{N}^{*}$ and $\Delta^{*}$ resonances that play a non-negligible role in the $\gamma p \rightarrow K \Sigma$ processes. This allows us to explore different sets of resonances with the same type of background. This type of background model contains a minimal number of free parameters but at the same time is considered physically acceptable with respect to the hadronic cutoff values and the SU(3) coupling constant restrictions. However, it will turn out that model D gives rise to results which are systematically inferior to those obtained with the three other models. This illustrates that compromising between the major features of model A, B and C does not necessarily lead to an improved description of the background diagrams.

\subsection{Reaction Dynamics}

When describing meson photoproduction, the different Feynman diagrams can in general be divided into two broad classes: the resonant part and the background. The resonant part consists exclusively of s-channel Feynman diagrams which contain resonant $\mathrm{N}^{*}$ and $\Delta^{*}$ particles in the intermediate state. By gradually increasing the photon energies, the s-channel resonances go through their respective poles and are expected to produce "structures" in the observables. Recent isobar models identified a set of three nucleon resonances as leading $N^{*}$ contributions to the $p(\gamma, K) Y$ processes $[25,27,29,54]$. This set of nucleon resonances consists of: $S_{11}(1650)$, $P_{11}(1710)$ and $P_{13}(1720)$. Characteristics of those and other resonant states are summarized in Table A.4 in App. A. In the forthcoming sections we will further discuss this selection. It will be shown that in some cases, the introduction of additional intermediate resonances may lead to substantial improvements in the quality of agreement between the calculations and the data.

In contrast to this resonant part, the background contains several classes of Feynman graphs. First the Born terms (the upper row in Fig. 2.1), involving an off-shell proton in the s-channel, a kaon exchange in the $t$-channel and hyperon exchange in the $u$-channel. Second, there are terms involving the exchange of (axial) vector meson resonances in the $t$-channel and $Y^{*}$ hyperon resonances in the $u$ channel. Despite the fact that in the latter case a resonant state is exchanged, they do not "resonate" in the same way as s-channel resonances do. Indeed, the poles in the $\mathrm{t}$ - or $\mathrm{u}$-channel are never reached in the physical region of the process and as 

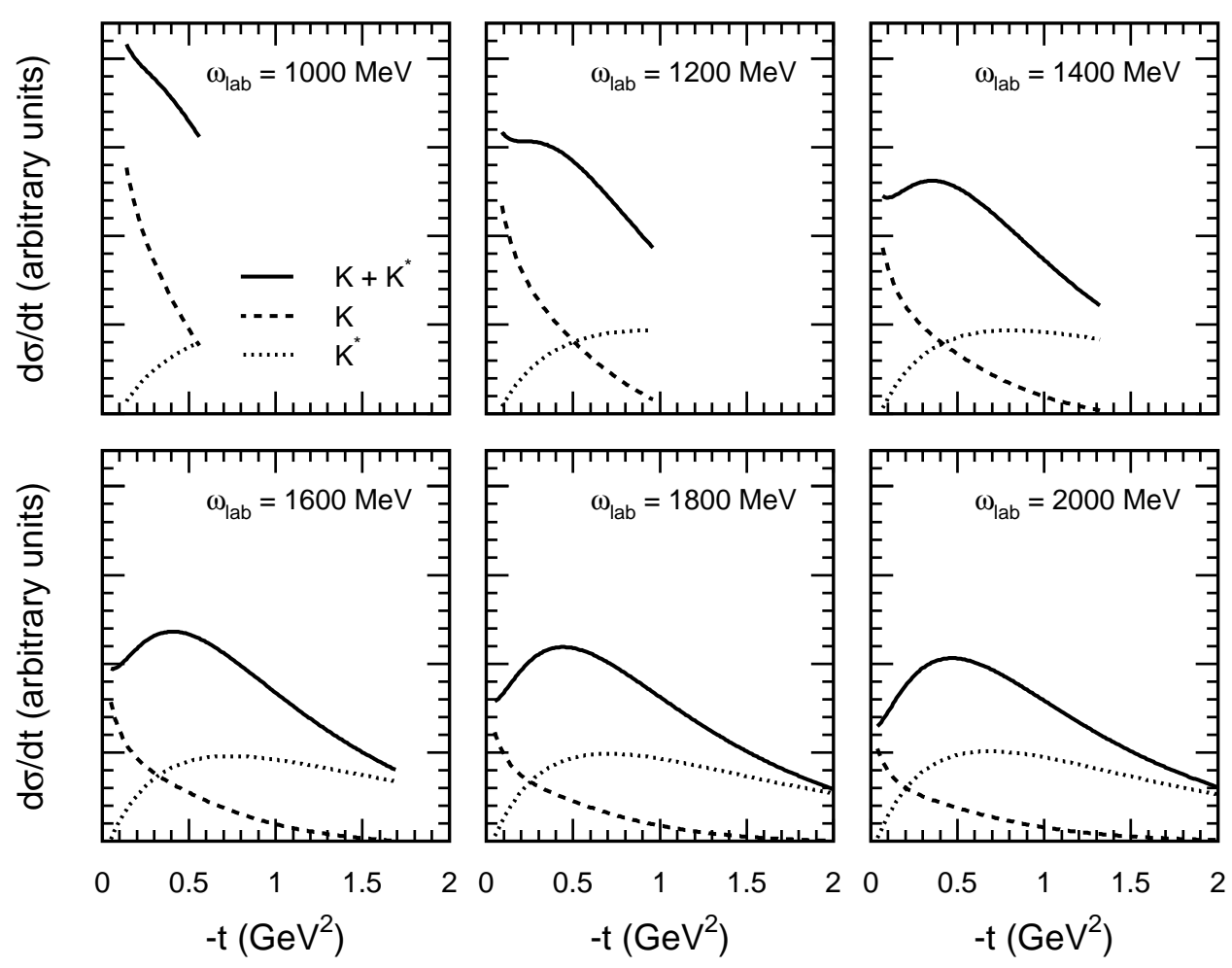

Figure 2.6 Contribution from the $t$-channel diagrams as a function of $t$ for various photon lab energies. The solid line is the combined strength of both the kaon and the $\mathrm{K}^{*}$ amplitude. The dashed and dotted curves are the result of the individual kaon and $\mathrm{K}^{*}$ exchange, respectively. In order to preserve gauge invariance, the kaon exchange term is extended with the nucleon pole term.

such are considered as background processes. In the $u$-channel, only $\mathrm{Y}^{*}$ hyperon resonances can be exchanged due to the conservation of the total strangeness quantum number $S$. This type of diagrams are only included in the background of model $B$ as an ingredient to reduce the Born strength. In the $t$-channel, the exchange of the vector meson $\mathrm{K}^{*}(892)$ is taken into account. The introduction is motivated by the well known observation that for meson photoproduction, the t-channel processes start dominating the reaction dynamics at higher energies [16]. This is reflected in a diffractive, forwardly peaked behavior of the differential cross sections. Even in the resonance region, the differential cross sections are observed to peak in the forward direction. This feature is usually interpreted in terms of kaon and $K^{*}$ exchange in 
the $\mathrm{t}$-channel. However, for some photon energies one discerns in both the $\mathrm{K}^{+} \wedge$ and the $\mathrm{K}^{+} \Sigma^{0}$ channel, differential cross sections which steeply rise as one moves out of the forward direction, reach their maximum and fall off again as more backward angles are probed $[23,24,55]$. The peak of the angular cross section does not coincide with exactly zero degrees and one experiences a short rise as one moves out of the very forward direction. This behavior can be understood as a subtle interference between the $\mathrm{K}$ and $\mathrm{K}^{*}$ diagrams. In Fig. 2.6 the calculated differential cross sections $\mathrm{d} \sigma / \mathrm{dt}$ are plotted as a function of $\mathrm{t}$ (note that forward angles correspond with low $t$ ) for various photon lab energies. For kaon exchange, a clear forward peaking emerges. Note that, in order to preserve gauge invariance, we have also added the nucleon $\mathrm{s}$-channel pole term. For the $\mathrm{K}^{*}$ vector meson, however, the operatorial form of the amplitude yields a vanishing strength at very forward angles. As can be seen in the figure, it is a balanced combination of both amplitudes that results in the observed non-forward peaking. For the $\mathrm{p}\left(\gamma, \mathrm{K}^{+}\right) \wedge$ reaction, also the exchange of the axial vector meson $K_{1}(1270)$ has been included in the description of the background. This axial vector meson has not the forward peaking behavior as its vector meson counterpart but the introduction seems to improve the overall description of the data. This meson is not found to be essential for the description of the $K \Sigma$ production reactions. As such, it is not included in those isospin channels. 


\section{Chapter 3}

\section{Strangeness Photoproduction}

In this chapter, we discuss our results for the strangeness photoproduction processes as obtained within the context of the model outlined in Chap. 2 and App. A. In Sec. 3.2, we focus on the $\gamma p \rightarrow K^{+} \wedge$ reaction. The isospin counterpart $\gamma p \rightarrow K \Sigma$ is discussed in Sec. 3.3. In Sec. 3.1, we review the observables, including those involving polarizations, which can be determined within the context of kaon photoproduction on the nucleon.

\subsection{Observables for KY Photoproduction}

We want to determine the cross section for a process whereby a photon $(\gamma)$ scatters on a proton $(\mathfrak{p})$, producing a kaon $(\mathrm{K})$ and a hyperon $(\mathrm{Y})$ in the finale state:

$$
p\left(p^{\mu}\right)+\gamma\left(k^{\mu}\right) \rightarrow K\left(p_{K}^{\mu}\right)+Y\left(p_{Y}^{\mu}\right) .
$$

In the center-of-mass frame, the four momenta read:

$$
\begin{array}{ll}
k^{\mu}=(\omega, \vec{k}), & p_{K}^{\mu}=\left(E_{K}, \vec{p}_{K}\right), \\
p^{\mu}=\left(E_{p},-\vec{k}\right), & p_{r}^{\mu}=\left(E_{Y},-\vec{p}_{K}\right) .
\end{array}
$$

The $z$-axis of the reference frame is chosen along the photon's three momentum and the $x z$-plane defines the reaction plane (see Fig. 3.1). The hadron energies are defined by the mass relations in the standard manner. The photon three momentum is given by $|\vec{k}|=\omega$ and the kaon three momentum $\left|\vec{p}_{\mathrm{K}}\right|$ is uniquely determined by the energy conservation relation:

$$
\sqrt{m_{\mathrm{p}}^{2}+\omega^{2}}+\omega=\sqrt{m_{\mathrm{K}}^{2}+\left|\vec{p}_{\mathrm{K}}\right|^{2}}+\sqrt{m_{Y}^{2}+\left|\vec{p}_{\mathrm{K}}\right|^{2}} .
$$




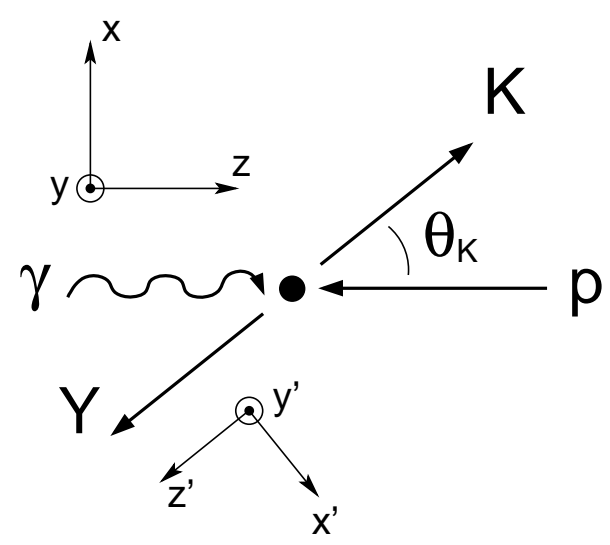

Figure 3.1 Orientation of the reference frames for the $p(\gamma, \mathrm{K}) \mathrm{Y}$ photoproduction process.

With these conventions, the differential cross section in the center-of-mass frame reads:

$$
\frac{\mathrm{d} \sigma}{\mathrm{d} \Omega_{\mathrm{K}}}=\frac{1}{64 \pi^{2}} \frac{\left|\overrightarrow{\mathrm{p}}_{\mathrm{K}}\right|}{\omega} \frac{1}{\mathrm{~W}^{2}} \overline{\sum_{\lambda_{\mathrm{i}} \lambda_{\mathrm{f}}} \lambda}\left|M_{\lambda}^{\lambda_{\mathrm{i}} \lambda_{\mathrm{f}}}\right|^{2},
$$

where $W \equiv \sqrt{s}$ is the invariant energy of the reaction and $s$ is defined as in Eq. (2.4). The reaction dynamics is entirely governed by the Feynman amplitude $M_{\lambda}^{\lambda_{i} \lambda_{f}}$ which depends on the photon $(\lambda)$, nucleon $\left(\lambda_{i}\right)$ and hyperon $\left(\lambda_{f}\right)$ polarizations. The explicit expression for this transition amplitude can be written as:

$$
\left|M_{\lambda}^{\lambda_{i} \lambda_{f}}\right|^{2}=\left(\bar{u}_{Y}^{\lambda_{f}}\left(p_{Y}\right) T^{\mu} \varepsilon_{\mu}^{\lambda} u_{p}^{\lambda_{i}}(p)\right)\left(\bar{u}_{p}^{\lambda_{i}}(p) \bar{T}^{v} \varepsilon_{v}^{\lambda *} u_{Y}^{\lambda_{f}}\left(p_{Y}\right)\right) .
$$

Here, $T^{\mu}$ is the current where the spinors of the external proton and hyperon field are removed. Further, $\bar{T}^{\mu}$ is defined as $\gamma^{0}\left(\mathrm{~T}^{\mu}\right)^{\dagger} \gamma^{0}$ and $\varepsilon_{\mu}^{\lambda}$ is the photon field polarization vector.

When the photon polarization remains unknown, the summation over the polarization $\lambda$ can be carried out using the expression:

$$
\sum_{\lambda= \pm 1} \varepsilon_{\mu}^{\lambda} \varepsilon_{v}^{\lambda *} \rightarrow-g_{\mu \nu}
$$

Remark that additional terms in the expression are required if the amplitude is not gauge invariant [56]. In addition, if the polarization of the participating hadrons remains undetected, the averaging and summation over the $\lambda_{i}$ and $\lambda_{f}$ spins reduce 
the Feynman amplitude to:

$$
\overline{\sum_{\lambda_{i} \lambda_{f} \lambda}}\left|M_{\lambda}^{\lambda_{i} \lambda_{f}}\right|^{2}=-\frac{1}{4} \operatorname{Tr}\left\{\left(\not p_{Y}+m_{Y}\right) T^{\mu}\left(\not p+m_{p}\right) \bar{T}_{\mu}\right\} .
$$

Here, we have made use of the spin sum rule $\sum_{\lambda} u^{\lambda}(q) \bar{u}^{\lambda}(q)=\not q+m$ of the Dirac algebra. It turns out that the determination of the dynamical part of the cross section can be reduced to computing a trace of $\gamma$-matrices. The calculation of this trace results in a closed expression for $|M|^{2}$ as a combination of the four vector products $(p \cdot k),\left(p_{K} \cdot k\right),\left(p \cdot p_{Y}\right) \ldots$.

When the polarization of the nucleon or the hyperon is specified in the reaction, a spin projection operator can be inserted in front of the appropriate spinor [56]:

$$
\Pi^{ \pm}(n)=\frac{1}{2}\left(1 \pm \gamma_{5} \not h\right)
$$

This still allows us to write the final expression for the polarized cross section as a trace of $\gamma$-matrices. In the rest frame of the polarized particle with momentum $p_{n}$, the projection four vector $n^{\mu}$ is defined as $n^{\mu}=(0, \vec{n})$ with $\vec{n}$ the spin quantization axis. Note that with this definition, $n^{2}=-1$ and $\left(n \cdot p_{n}\right)=0$ in any other frame. Due to the occurrence of an additional $\gamma_{5}$-matrix in the trace, the result of the trace calculation now also contains determinants of the type $\operatorname{det}\left(k, p, p_{k}, n\right)$.

Polarized particles give rise to asymmetries. The asymmetry observables and their particular quantization axes are summarized in Table 3.1. The axes are depicted in Fig. 3.1. These asymmetries are defined in the standard manner:

$$
\text { asymmetry }=\frac{d \sigma^{+}-d \sigma^{-}}{d \sigma^{+}+d \sigma^{-}},
$$

where the $+(-)$ refers to a polarization parallel (anti-parallel) with the respective quantization axis or helicity state (in the case of circularly polarized photons). Double polarization asymmetries are defined as:

$$
\text { asymmetry }=\frac{d \sigma^{(++)}+d \sigma^{(--)}-d \sigma^{(+-)}-d \sigma^{(-+)}}{d \sigma^{(++)}+d \sigma^{(--)}+d \sigma^{(+-)}+d \sigma^{(-+)}} .
$$

One can choose the quantization axes such that: $d \sigma^{(++)}=d \sigma^{(--)}$and $d \sigma^{(+-)}=$ $\mathrm{d} \sigma^{(-+)}$. Eq. (3.10) can then be rewritten as:

$$
\begin{aligned}
\text { asymmetry } & =\frac{d \sigma^{(++)}-d \sigma^{(+-)}}{d \sigma^{(++)}+d \sigma^{(+-)}}, \\
& =\frac{d \sigma^{(--)}-d \sigma^{(-+)}}{d \sigma^{(--)}+d \sigma^{(-+)}} .
\end{aligned}
$$




\begin{tabular}{|c|c|c|c|}
\hline Observable & $\gamma$ & $p$ & $\Lambda$ \\
\hline $\begin{array}{c}\mathrm{d} \sigma / \mathrm{d} \Omega \\
\Sigma \\
\mathrm{T} \\
\mathrm{P}\end{array}$ & $l$ & $y$ & $y^{\prime}$ \\
\hline $\begin{array}{c}\text { beam-target } \\
\text { E } \\
\text { F } \\
\text { G } \\
\text { H }\end{array}$ & $\begin{array}{l}c \\
c \\
t \\
t\end{array}$ & $\begin{array}{l}-z \\
-x \\
-z \\
-x\end{array}$ & \\
\hline $\begin{array}{c}\text { beam-recoil } \\
\mathrm{C}_{x} \\
\mathrm{C}_{z} \\
\mathrm{O}_{x} \\
\mathrm{O}_{z}\end{array}$ & $\begin{array}{l}c \\
c \\
t \\
t\end{array}$ & & $\begin{array}{l}x^{\prime} \\
z^{\prime} \\
x^{\prime} \\
z^{\prime}\end{array}$ \\
\hline $\begin{array}{c}\text { target-recoil } \\
\mathrm{T}_{x} \\
\mathrm{~T}_{z} \\
\mathrm{~L}_{x} \\
\mathrm{~L}_{z}\end{array}$ & & $\begin{array}{l}-x \\
-x \\
-z \\
-z\end{array}$ & $\begin{array}{l}x^{\prime} \\
z^{\prime} \\
x^{\prime} \\
z^{\prime}\end{array}$ \\
\hline
\end{tabular}

Table 3.1 Definition of the photoproduction observables. The quantization axes for the polarization asymmetries are defined as follows: $\vec{z} \sim \vec{k}, \vec{y} \sim\left(\vec{k} \times \vec{p}_{k}\right), \vec{x}=\vec{y} \times \vec{z}, \vec{z}^{\prime} \sim \vec{p}_{\wedge}$, $\vec{y}=\vec{y}^{\prime}, \vec{x}^{\prime}=\vec{y}^{\prime} \times \vec{z}^{\prime} . \quad l \rightarrow$ linearly polarized photon $(\perp, \|$ with respect to scattering plane); $\mathrm{t} \rightarrow$ linearly polarized photon $( \pm \pi / 4$ with respect to scattering plane); $\mathrm{c} \rightarrow$ circularly polarized photon. The axes are also shown in Fig. 3.1 
It is well known that the sixteen quantities, as given in Table 3.1, are not independent. There exist six non-linear relations between them [10,57]:

$$
\begin{aligned}
& \mathrm{E}^{2}+\mathrm{F}^{2}+\mathrm{G}^{2}+\mathrm{H}^{2}=1+\mathrm{P}^{2}-\Sigma^{2}-\mathrm{T}^{2}, \\
& \mathrm{FG}-\mathrm{EH}=\mathrm{P}-\Sigma \mathrm{T}, \\
& \mathrm{C}_{x}^{2}+\mathrm{C}_{z}^{2}+\mathrm{O}_{x}^{2}+\mathrm{O}_{z}^{2}=1+\mathrm{T}^{2}-\mathrm{P}^{2}-\Sigma^{2}, \\
& \mathrm{C}_{z} \mathrm{O}_{x}-\mathrm{C}_{x} \mathrm{O}_{z}=\mathrm{T}-\mathrm{P} \Sigma, \\
& \mathrm{T}_{x}^{2}+\mathrm{T}_{z}^{2}+\mathrm{L}_{x}^{2}+\mathrm{L}_{z}^{2}=1+\Sigma^{2}-\mathrm{P}^{2}-\mathrm{T}^{2}, \\
& \mathrm{~T}_{x} \mathrm{~L}_{z}-\mathrm{T}_{z} \mathrm{~L}_{x}=\Sigma-\mathrm{PT},
\end{aligned}
$$

which reduce the number of independent observables necessary to determine the full transition amplitude.

\subsection{K^ Photoproduction}

In this section, we present our results on the $\mathrm{K}^{+} \wedge$ photoproduction reaction. First, we discuss the resonant and background contributions. Further on, we focus on the role of hadronic form factors and conclude with some remarks on the issue of the missing resonances.

\subsubsection{Resonance Contributions}

In our selection of the intermediate $\mathrm{N}^{*}$ particles contributing to the $\mathrm{p}\left(\gamma, \mathrm{K}^{+}\right) \wedge$ process, we have been guided by a recent coupled-channel analysis [25]. This study established the importance of three intermediate nucleon states: two spin-1/2 resonances $\left(S_{11}(1650)\right.$ and $\left.P_{11}(1710)\right)$ and one spin-3/2 resonance $\left(P_{13}(1720)\right)$. These findings are confirmed by the isobar model calculations of Refs. [27,29]. Those nucleon resonances are also the only ones in the tables of the Particle Data Group with significant branching into the strange channels [58]. In our numerical calculations, these three resonances $\left(S_{11}(1650), P_{11}(1710), P_{13}(1720)\right)$ constitute the "core" of the reaction dynamics. In order to confront this set of intermediate states with the data, a particular background has to be constructed. In a naive approach, we would only 
include the Born terms (modified with a hadronic form factor with a hard cutoff, $\Lambda=1.6 \mathrm{GeV}$ ) and the $\mathrm{K}^{*}$ and $\mathrm{K}_{1}$ meson exchange contributions. An attempt to fit the $\mathrm{K}^{+} \Lambda$ cross section and polarization data with this naive background and the core set of three $\mathrm{N}^{*}$ resonances was not very successful. Indeed, the agreement did not get any better than $\chi^{2}=10.32$ (see Table 3.2). This $\chi^{2}$ expresses the conformity of the model calculations to the data. A value about 10 clearly indicates that there is room for improvements. The most important reason for the failure of this calculation is the absence of a proper strategy to counterbalance the strength stemming from the Born terms (see discussion in Sec. 2.3.2). Indeed, adopting the scheme of model A or B to handle the background contributions, a considerable improvement of the overall agreement of the model calculations with the data is observed. $\chi^{2}$ values of 4.36 and 3.43 were obtained for the models A and B, respectively. This improvement in the quality of the description clearly illustrates the need for a proper description of the background diagrams.

The research in this field has experienced a new impulse with the advent of the $p\left(\gamma, \mathrm{K}^{+}\right) \wedge$ data from the SAPHIR experiment at Bonn [23]. These data provide some indications for a structure in the total $p\left(\gamma, K^{+}\right) \wedge$ cross section about $\omega_{\mathrm{lab}}=$ $1.5 \mathrm{GeV}$. Due to limited energy resolution and statistics, this structure could not be revealed in previous experiments. Recently, the George Washington group pointed out that model calculations could account for this structure in the measured cross sections after including an additional spin-3/2 nucleon resonance ( $\left.\mathrm{D}_{13}(1895)\right)$ in the s-channel [27]. This $\mathrm{D}_{13}$ state has never been observed in pionic reactions but the existence of this resonance with considerable branching into the strange channel, was predicted by the constituent quark model calculations of Capstick and Roberts [4]. Therefore, the authors of Ref. [27] legitimately claimed support for the existence of one of the "missing resonances". Our calculations confirm the conclusions drawn by the G. Washington group. When including the $\mathrm{D}_{13}(1895)$ in addition to the core set of $\mathrm{N}^{*}$ resonances, we arrive at a promising $\chi^{2} \simeq 2.9$ (see Table 3.2). Again, the necessity of a realistic description of the background terms is observed. Within model A, B or C for describing the background, comparable $\chi^{2}$ values are obtained. When no attempts are made to cut the Born strength, this means that no $Y^{*}$ resonances are introduced in the $u$-channel and a hard cutoff value $\Lambda \geq 1.6 \mathrm{GeV}$ is adopted in the calculations, a $\chi^{2}$ no better than 9.74 is reached. 


\begin{tabular}{|c|c|c|c|c|}
\hline \multicolumn{2}{|c|}{ Resonance Contributions } & \multirow{2}{*}{$\begin{array}{c}\text { Background } \\
\text { Model }\end{array}$} & \multirow[t]{2}{*}{$\overline{x^{2}}$} & \multirow[t]{2}{*}{$\overline{N N F P}$} \\
\hline $\mathrm{N}^{*}$ core set & $\mathrm{D}_{13}(1895)$ & & & \\
\hline 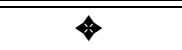 & & - & $10.32^{\dagger}$ & 16 \\
\hline$\downarrow$ & & A & $4.36^{\dagger}$ & 16 \\
\hline$\downarrow$ & & B & $3.43^{\dagger}$ & 18 \\
\hline$\downarrow$ & $\downarrow$ & - & 9.74 & 20 \\
\hline$\checkmark$ & $\checkmark$ & $\mathrm{A}$ & 2.99 & 20 \\
\hline$\downarrow$ & $\checkmark$ & B & 2.89 & 22 \\
\hline 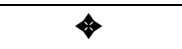 & $\checkmark$ & $\mathrm{C}$ & 2.85 & 20 \\
\hline
\end{tabular}

Table 3.2 The table summarizes the $\chi^{2}$ values for the different combinations of $\mathrm{N}^{*}$ resonances with a particular type of background as obtained from comparing the model calculations with the $p\left(\gamma, \mathrm{K}^{+}\right) \wedge$ SAPHIR data. With " $\mathrm{N}^{*}$ core set" we refer to the $S_{11}(1650)$, $S_{13}(1710)$ and $P_{13}(1720)$ nucleon resonances. "NFP" indicates the number of free parameters in the fitting procedure. The $\chi^{2}$ values with a $\dagger$ are obtained with the Haberzettl recipe for the gauge restoration procedure. If the background model is indicated with "-", no attempts have been made to cut down the Born strength. Only a cutoff value of $\Lambda \geq 1.6 \mathrm{GeV}$ is imposed for the Born terms.

After all, we can conclude that the core set of $S_{11}(1650), P_{11}(1710), P_{13}(1720)$, extended with the $\mathrm{D}_{13}(1895)$ resonance is able to reproduce the $\mathrm{K}^{+} \wedge$ photoproduction data from the SAPHIR collaboration, as long as the background contributions are properly taken into account.

\subsubsection{Background Contributions}

As detailed in Sec. 2.3.2 and also numerically observed in the previous Sec. 3.2.1, an effective Lagrangian approach to the $p\left(\gamma, \mathrm{K}^{+}\right) \wedge$ process requires additional mechanisms to counterbalance the unreasonable amounts of strength arising from pointlike Born terms. We have performed model calculations along the lines of the three different techniques to deal with the background diagrams described in the Sec. 2.3.2. We refer to those three different treatments as the models A, B and C and their major features are summarized in Table 2.1.

- Model A: In this model, the background is restricted to the Born terms and $t$-channel diagrams involving the $K^{*}$ vector meson and $K_{1}$ axial vector meson exchange. The strength of the Born diagrams is primarily tempered by the action of hadronic form factors. During the fitting procedure, we imposed 

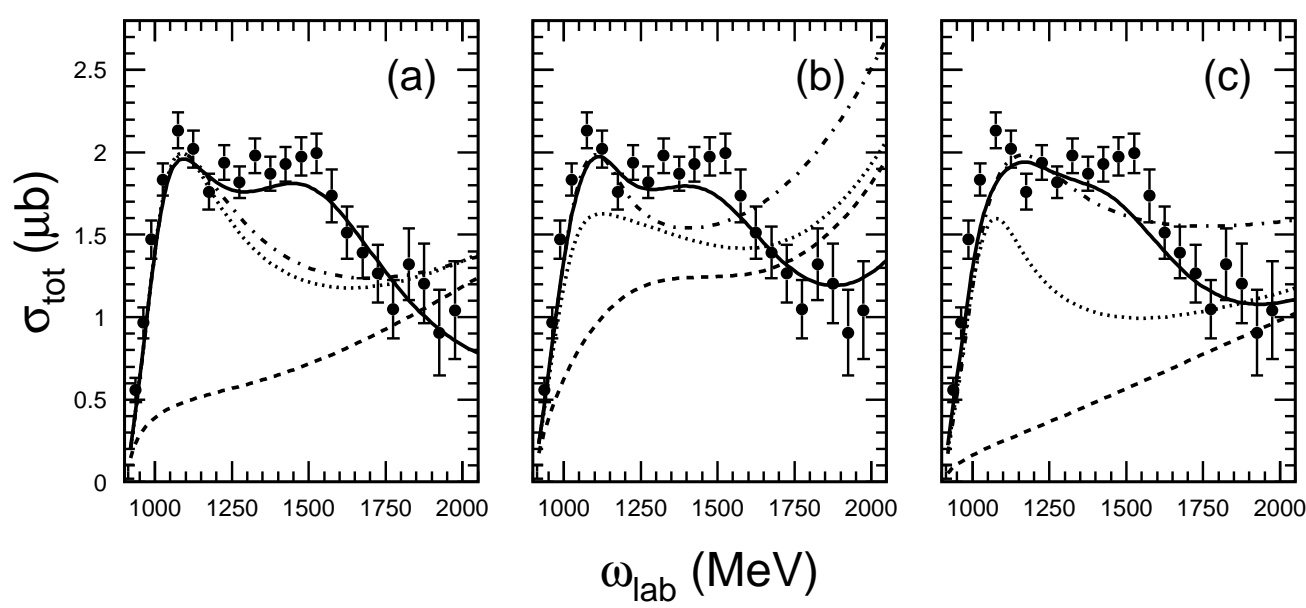

Figure 3.2 The total $\mathrm{p}\left(\gamma, \mathrm{K}^{+}\right) \wedge$ cross section versus the photon lab energy as obtained with three different techniques to treat the background contributions. Panel (a), (b) and (c) use model A, B and C respectively. In each panel, the contribution from the background terms to the total cross sections is denoted by the dashed line. In addition to the background terms, the dotted line includes the $S_{11}(1650)$ and the $P_{11}(1710)$ nucleon resonances. The dot-dashed curve adds also the $\mathrm{P}_{13}(1720)$ resonance. Finally, for the solid line also the $\mathrm{D}_{13}(1895)$ resonance is included. The data are from Ref. [23].

an under limit of $0.4 \mathrm{GeV}$ for the (freely varying) value of the cutoff mass $\Lambda$. It emerges that the best fits to the data are obtained with values of $\Lambda$ that approach this imposed under limit, corresponding with an extremely soft hadronic form factor. As can be seen in Fig. 3.2a, the photon energy dependence of the background is smooth and steadily rising. Concerning the contributions from the resonant terms in model $\mathrm{A}$, the strength produced by the $P_{13}(1720)$ is rather small and the structure about photon lab energies of 1.5 $\mathrm{GeV}$ is clearly dominated by the $\mathrm{D}_{13}(1895)$. Despite the fair agreement with the data reached in model A $\left(\chi^{2}=2.99\right)$, one can raise serious doubts about the realistic character of cutoff masses as small as the kaon mass. Indeed, a form factor represents a purely phenomenological description of the shortrange dynamics of the inter-baryon interaction and sets a short-distance scale beyond which the theory is believed to fail. With cutoff masses approaching the kaon mass, the form factor will unavoidably start playing a predominant role in the theoretical description of the reaction dynamics, which is a rather 
unsatisfactory situation for an effective theory.

- Model B: Here, we have extended the background (Born terms plus $\mathrm{K}^{*}$ and $\mathrm{K}_{1}$ $t$-channel exchange) with two $\Lambda^{*}$ resonances $\left(S_{01}(1800)\right.$ and $\left.P_{01}(1810)\right)$ in the $u$-channel. Through destructive interference, the total background strength gets reduced to acceptable levels (see Fig. 3.2b), a virtue which is now reached with realistic values of the cutoff mass of the order $1.5 \mathrm{GeV}$. The overall agreement with the SAPHIR data set is $\chi^{2}=2.89$. The hyperon coupling constants which arise from the fits are relatively large $\left(G_{\Lambda^{*}(1800)}=-4.38\right.$ and $G_{\Lambda^{*}(1810)}=-$ 1.75 ) and can be subject to discussion. To clarify this issue, we have performed fits to the data using a model which introduces seven spin-1/2 hyperon resonances in the $u$-channel. The same qualitative destructive interference effect was observed but now with smaller values for $G_{Y^{*}}$. In the light of these findings, we argue that the two hyperon resonances which were introduced in model B could be interpreted as effective particles which account for a larger set of hyperon resonances participating in the process. Note that $u$-channel resonances do not reach their pole in the physical region of the process and consequently exhibit a smooth energy behavior. From Fig. 3.2 it becomes clear that the final result for the total cross section, calculated in model B, displays a more complicated pattern than what is typically observed for model A. Whereas model A predicts that the resonances peak at their corresponding invariant masses, in model B a rather complex interference pattern (especially at higher photon energies) between the different resonances appears.

- Model C: As a third option for controlling the magnitude of the background contributions, we have performed a set of fits to the data where we ignored the restrictions of Eq. (2.11) imposed by broken SU(3)-flavor symmetry. We only put limitations on the signs of $g_{K^{+} \wedge p}$ and $g_{K^{+} \Sigma^{0}}$. Completely analogous as model $\mathrm{A}$, in model $\mathrm{C}$ the background consists of the Born diagrams and the two spin-1 t-channel contributions. An under limit of $1.1 \mathrm{GeV}$ was imposed for the Born term form factor cutoff mass but during the fit, $\Lambda$ arrived at a rather hard value of $1.85 \mathrm{GeV}$. Also within this model, the data can be reasonably well described $\left(\chi^{2}=2.85\right)$. Nevertheless, the overall best fit was obtained for a value $g_{K^{+} \wedge p} / \sqrt{4 \pi}=-0.40$ which is far below the $S U(3)$ prediction of -3.75 . 
All three techniques to deal with the background terms, eventually lead to a fair agreement of the model calculations with the available data. To illustrate this, Table 3.2 summarizes the $\chi^{2}$ per degree-of-freedom obtained in the three models. The model predictions for the differential cross sections and the recoil polarization asymmetries are displayed in Figs. 3.3 and 3.4. A complete list of all numerical input parameters of the three models can be found in Table A.2 in App. A. Despite the fact that the $\chi^{2}$ values are comparable and uniform results are observed for the cross sections and recoil asymmetries, Fig. 3.5 clearly shows that the extracted values for the $\mathrm{N}^{*}$ coupling constants (as defined in App. A) differ drastically in the three models. From this observation, we draw the conclusion that the model assumptions, with respect to the treatment of the background terms, heavily influence the extracted information about the resonances. Remarkably, it appears that the choices made with respect to modeling the background terms not only affect the magnitude of the different $\mathrm{N}^{*}$ contributions, but also the interference pattern between the overlapping resonances (see Fig. 3.2). Through the $\chi^{2}$ minimization procedure, though, the final agreement between the numerical calculations and the data appears reasonable in all models to deal with the background.

One may now look at the predictive power of these models for observables for which no data exist to date. Such an observable, for example, is the photon beam asymmetry $(\Sigma)$. Fig. 3.6 summarizes the predictions for the photon beam asymmetries as a function of the photon lab energy and $\cos \theta$ for the three background models. In addition, we also have plotted the corresponding differential cross sections over the same phase space. Visual inspection learns that the computed differential cross sections do not vary that much as one proceeds from one background model to another. A completely different picture is observed for the calculated photon beam asymmetries. Dramatic variations in $\cos \theta$ and $\omega_{l a b}$ are perceived between the three background models. In Fig. 3.7, the same erratic behavior is observed in the angular distribution of the double polarized observables as the beam-recoil $\left(\mathrm{O}_{x}\right)$ and beam-target $(\mathrm{E})$ asymmetry. Recent measurements of the single photon beam asymmetry $\Sigma$ at GRAAL [59] and SPring-8 [60] will in this light be a first important step to distinguish between the three background models and further pin down the details of the underlying reaction dynamics. 


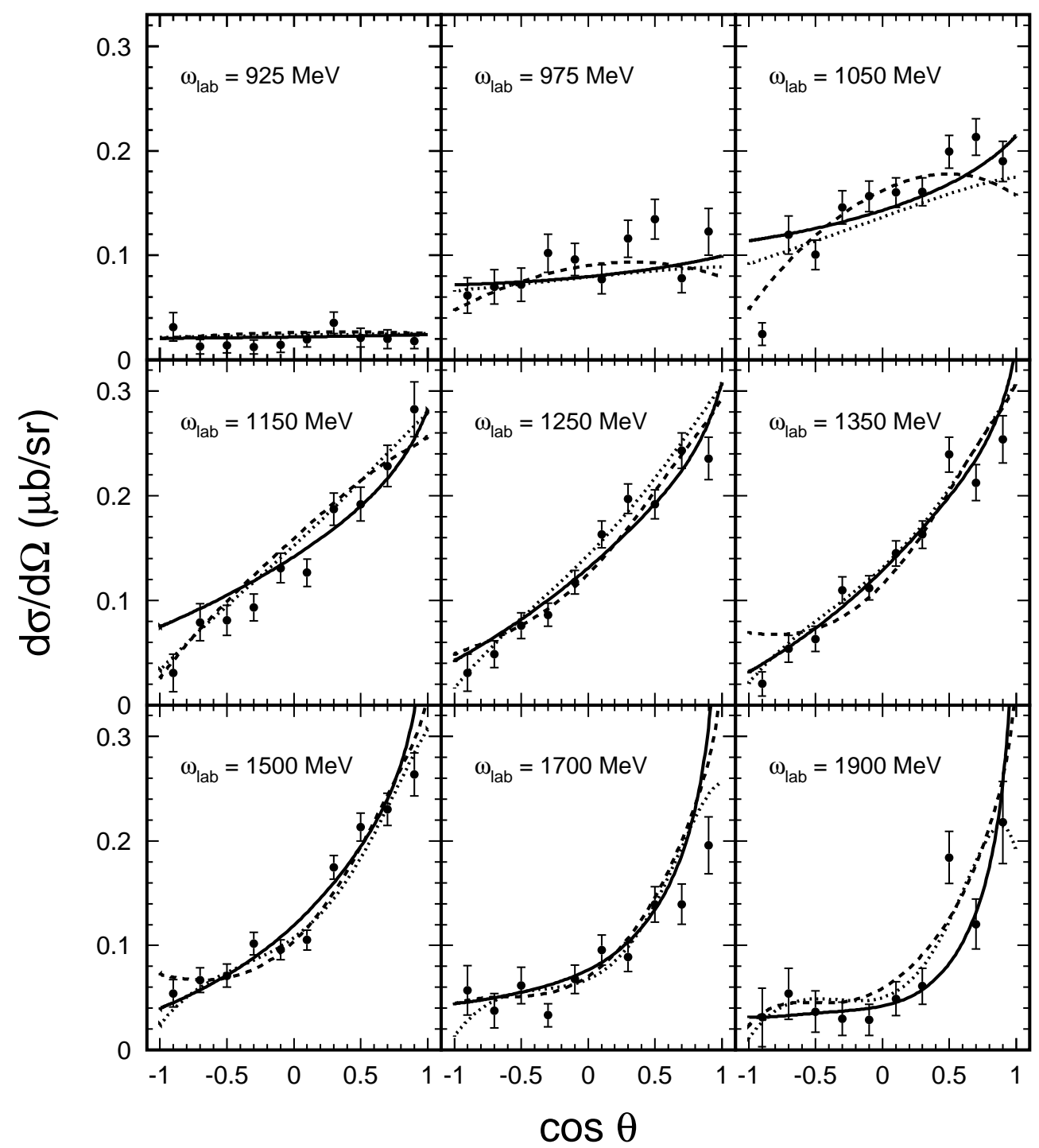

Figure 3.3 Model calculations of the angular distribution of the differential $p\left(\gamma, K^{+}\right) \wedge$ cross sections for various photon lab energies. The solid, dashed and dotted curves are obtained with the background models A, B and C, respectively. The data are from Ref. [23]. 


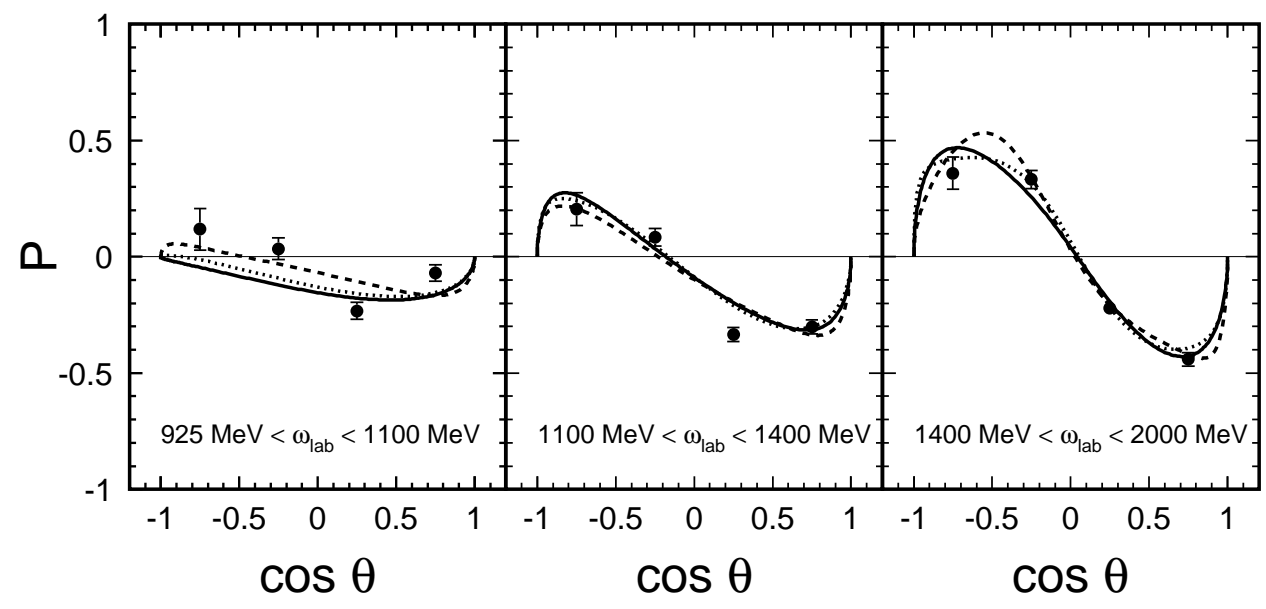

Figure 3.4 Model calculations of the angular distribution of the $p\left(\gamma, \mathrm{K}^{+}\right) \vec{\Lambda}$ recoil polarization asymmetry for various photon lab energies. Line conventions are as in Fig. 3.3. The results are averaged over the experimental energy bins. The data are from Ref. [23].

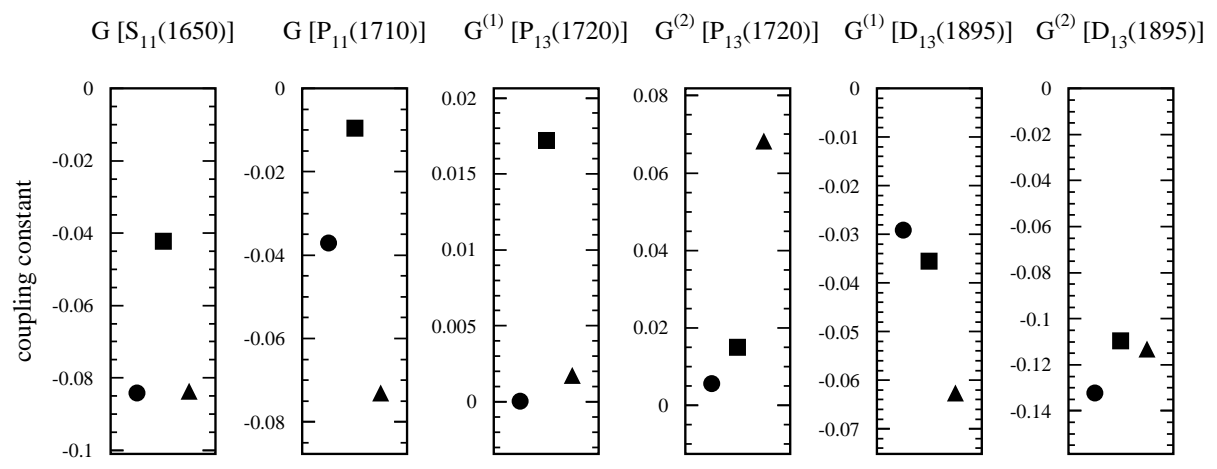

Figure 3.5 The extracted coupling constants for the $S_{11}(1650), P_{11}(1710), P_{13}(1720)$ and $\mathrm{D}_{13}$ (1895) s-channel resonances, using the three different models for dealing with the background terms. The circles are for model A, the squares for model B and the triangles for model C. The coupling constants are defined in App. A. 

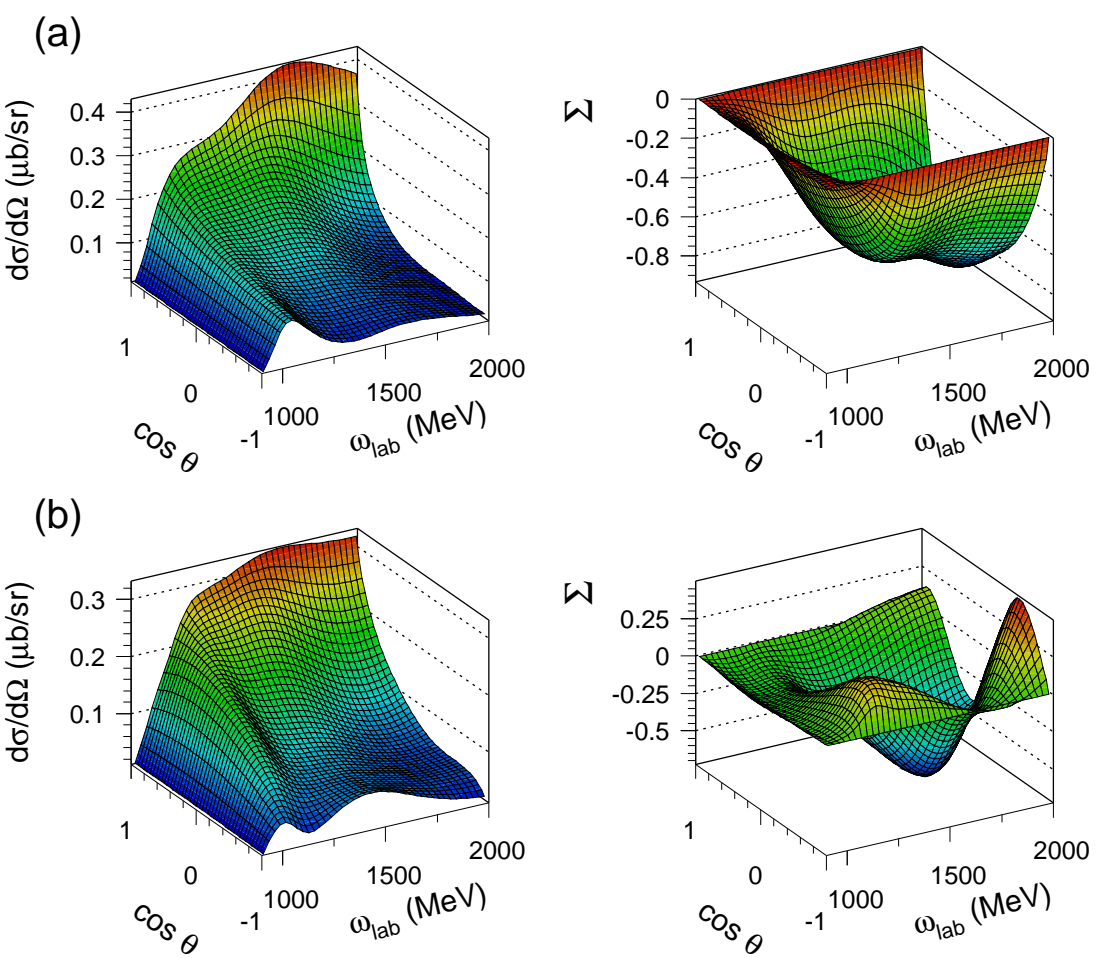

(c)
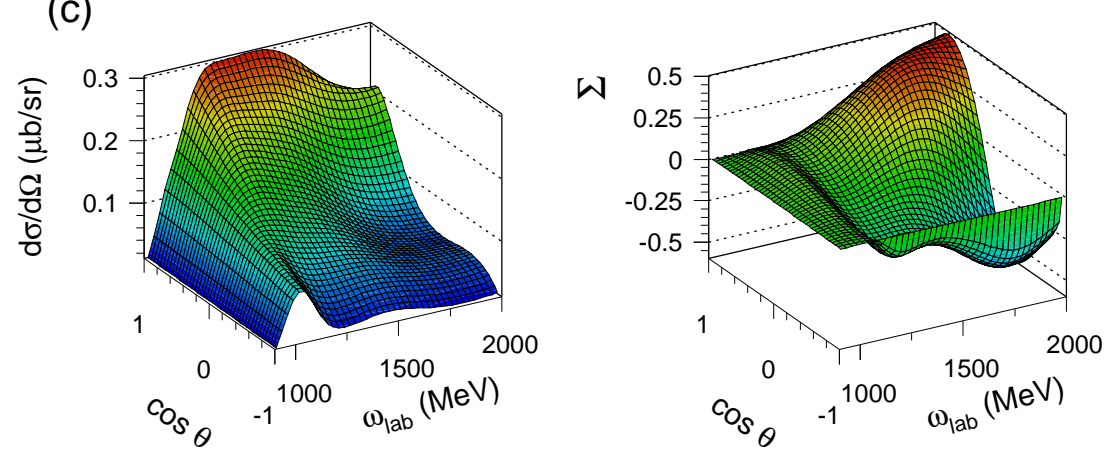

Figure 3.6 Differential cross sections and photon beam asymmetries for the $p\left(\gamma, K^{+}\right) \wedge$ process as a function of the photon lab energy and $\cos \theta$. All calculations account for the same set of s-channel resonances but adopt a different implementation of the background terms. Panels (a), (b) and (c) contain model calculations performed within model A, B and C, respectively. 

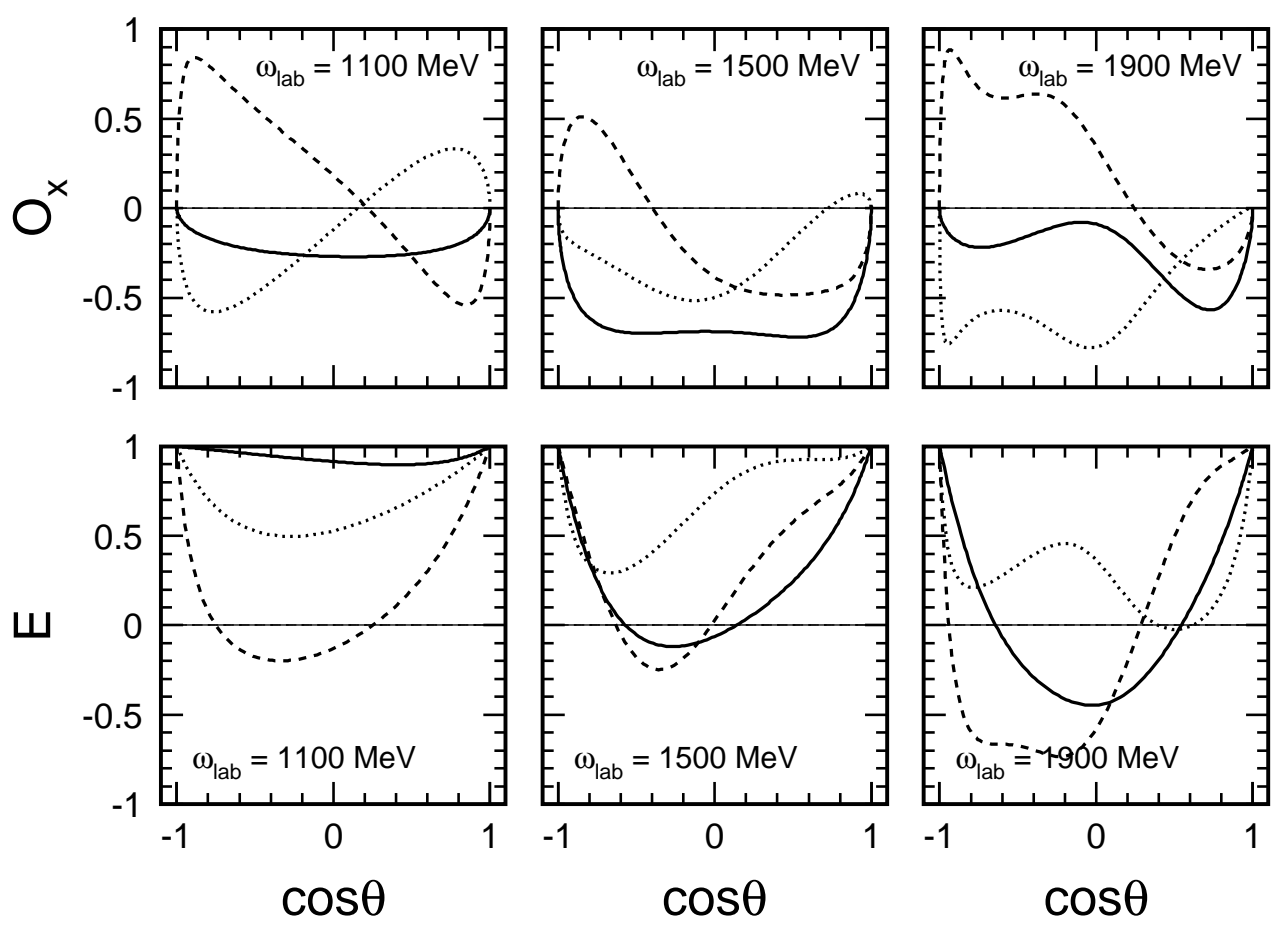

Figure 3.7 Angular distribution for the $\mathrm{p}\left(\vec{\gamma}, \mathrm{K}^{+}\right) \vec{\Lambda}$ beam-recoil $\left(\mathrm{O}_{x}\right)$ and the $\vec{p}\left(\vec{\gamma}, \mathrm{K}^{+}\right) \wedge$ beam-target $(E)$ polarization asymmetry for three different photon lab energies. The specific quantization axes of the polarized particles are given in Table 3.1. Line conventions for the model calculations are as in Fig. 3.3. 
To conclude, we make the following remark. In addition to the three frameworks to deal with the background diagrams presented here, one could think of an alternate method to improve the overall agreement with the data. Other $\mathrm{N}^{*}$ resonances beyond the set consisting of $S_{11}(1650), P_{11}(1710), P_{13}(1720)$ and $D_{13}(1895)$, could be introduced as likely candidates for playing a significant role in the reaction dynamics of $\mathrm{p}\left(\gamma, \mathrm{K}^{+}\right) \wedge$. We have performed calculations introducing additional spin-1/2 $\mathrm{N}^{* \prime} \mathrm{s}$ in the s-channel, but keeping the number of additional free parameters in the fitting procedure at a reasonable level. In these computations, the cutoff mass $\Lambda$ was forced to adopt (realistic) values larger than $1.1 \mathrm{GeV}$. However, none of the numerical calculations reached a $\chi^{2}$ better than 8 (which has to be compared to typical values of $\chi^{2} \simeq 2.9$ produced by the other models). In other words, the introduction of additional resonances in the s-channel can not be invoked as a viable mechanism for cutting down the background strength and improve the description.

\subsubsection{Hadronic Form Factors}

Up to now, in all numerical calculations we have used the gauge restoration procedure as suggested by Davidson and Workman [46]. This recipe is outlined in Sec. 2.2. However, in the literature the Haberzettl form [45] for the form factor $\widehat{F}$ is frequently adopted. In this section, we explore the implications of this two different prescriptions. In that respect, we have performed numerical calculations for the $p\left(\gamma, K^{+}\right) \wedge$ reaction using both the $\widehat{F}_{H}$ and the $\widehat{F}_{D W}$ functional form in the contact term. Those form factors are defined in Eqs. (2.7) and (2.9). For those numerical calculations using $\widehat{F}_{H}$, we have put $a_{\mathfrak{u}}=0$. This choice is motivated by the observation that in the $p\left(\gamma, \mathrm{K}^{+}\right) \wedge$ process, the gauge violating terms only occur in the sand $t$-channel. As a result, calculations using the $\widehat{F}_{H}$ form have two remaining free parameters $\left(\Lambda\right.$ and $a_{s}$ ). In practice, we found that the best fits were obtained for $a_{s} \simeq 1$ and accordingly $\widehat{F}_{H} \simeq F_{s}(\Lambda)$. Fig. 3.8 compares the values of $\widehat{F}_{H}$ and $\widehat{F}_{D W}$ at various photon energies $\omega_{\mathrm{lab}}$ and kaon center-of-mass angles $\theta$. The left panels show the form factors for a cutoff mass $\Lambda=0.8 \mathrm{GeV}$, the right panels use $\Lambda=1.8$ $\mathrm{GeV}$. They are representative for a rather soft and hard option for the form factors, respectively. It becomes obvious from Fig. 3.8 that the magnitude of the form factor $\widehat{F}$ heavily depends on the adopted recipe, both in the soft and hard limit for the 

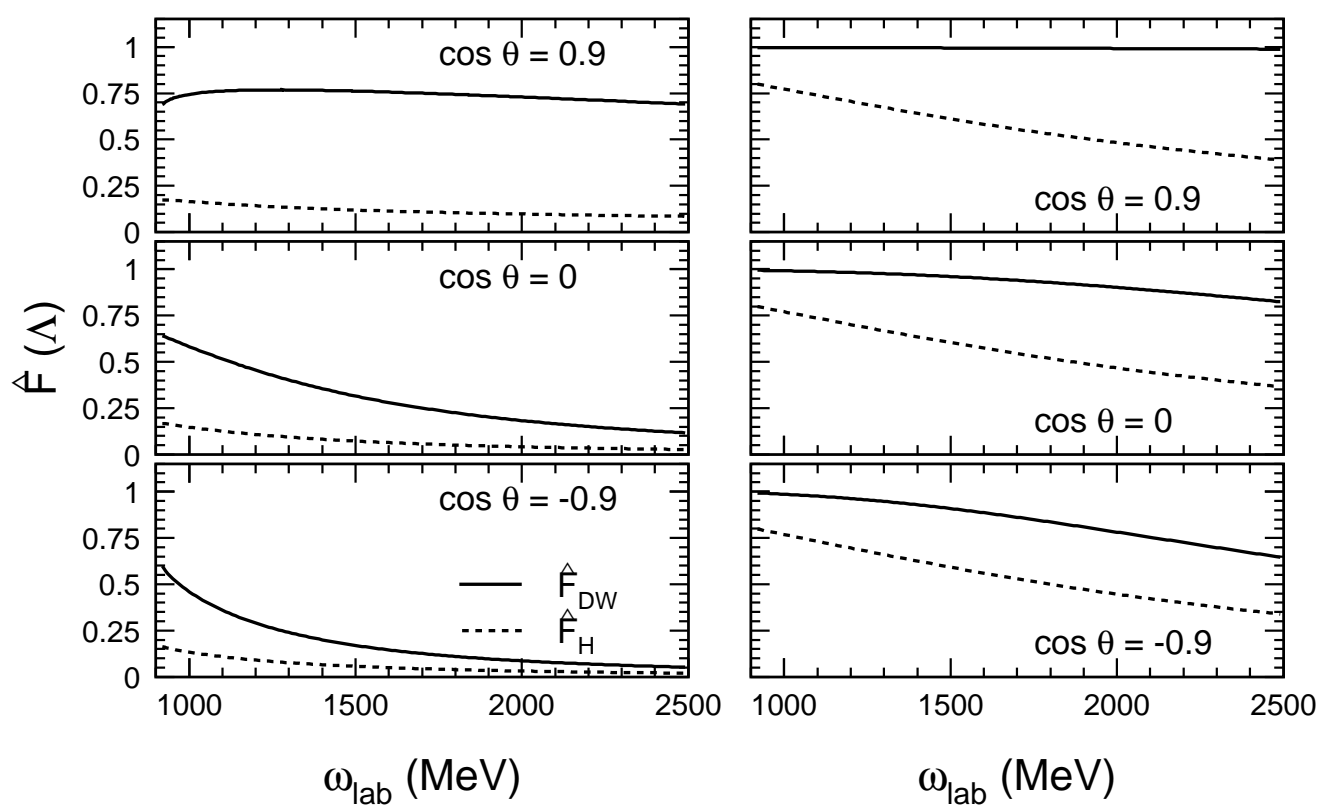

Figure 3.8 The energy dependence of the hadronic form factor $\widehat{F}$ for different kaon centerof-mass angles $\theta$. The left panels use $\Lambda=0.8 \mathrm{GeV}$, the right panels $\Lambda=1.8 \mathrm{GeV}$. The dashed line is from the $\widehat{F}_{H}$ Haberzettl form (with $a_{s}=0.9$ ). The solid lines represent the form $\widehat{F}_{D W}$ proposed by Davidson and Workman.

\section{cutoff mass.}

In order to assess the sensitivity of the results to differences in the functional form of $\widehat{F}$, we have computed $p\left(\gamma, K^{+}\right) \Lambda$ observables using the two forms for $\widehat{F}$. Both recipes resulted in a comparable agreement with the data and from the obtained $\chi^{2}$ values, no conclusions can be drawn. However, the results for the extracted $\mathrm{N}^{*}$ coupling constants are given in Fig. 3.9 for the background models $\mathrm{A}$ and $\mathrm{B}$. In model A, where by construction a large role in the reaction dynamics is attributed to form factors, the effect is huge. In model B, where hyperon resonances are introduced to counterbalance the strength from the Born terms and hadronic form factors are not so dominant, the extracted coupling constants are a little more stable against variations in the functional dependence of $\widehat{F}$, although also here sizable variations are observed. A similar trend is for example also seen in the photon beam asymmetry in Fig. 3.10. Whereas for model A different choices for $\widehat{F}$ even 


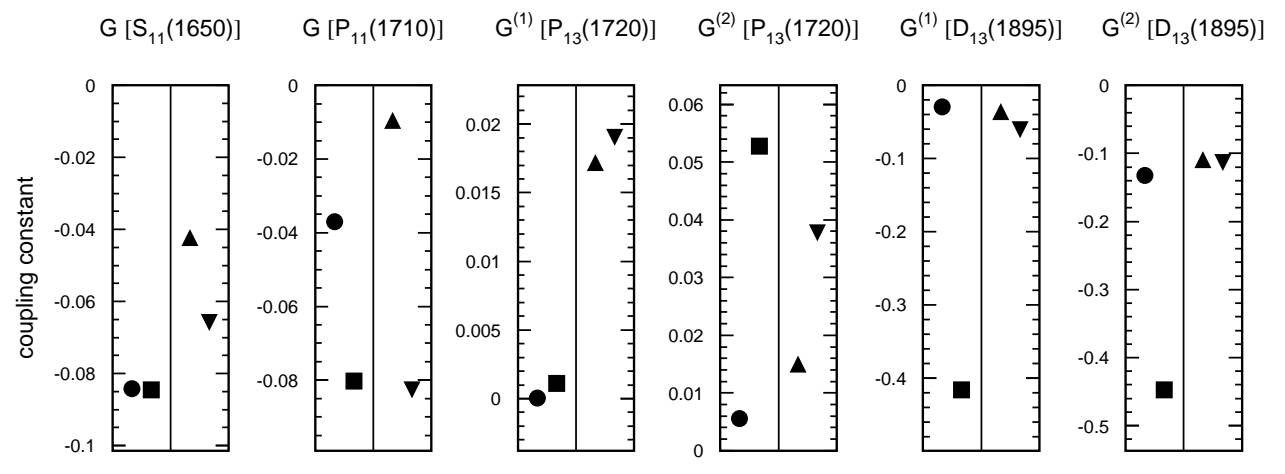

Figure 3.9 The sensitivity of the extracted $\mathrm{N}^{*}$ coupling constants to the adopted form for the hadronic form factor $\widehat{F}$. The circles are obtained from calculations using the $\widehat{F}_{D w}$ form, the squares with $\widehat{\mathrm{F}}_{\mathrm{H}}$. They both correspond to a calculation which uses model $\mathrm{A}$ to treat the background contributions. Analogously, the triangles $\boldsymbol{\Delta}(\boldsymbol{\nabla})$ are for the $\widehat{F}_{D W}\left(\widehat{F}_{H}\right)$ form in model B.

flip the sign of the predicted asymmetry, in model B the situation looks reasonably stable. Only at the highest photon energies considered here, the predicted asymmetry in model B becomes sensitive to the adopted recipe for the form factor in the contact term.

These conclusions are compatible with earlier observations concerning the difference between the form factor prescriptions of Ohta and Haberzettl. Ohta originally suggested to put the form factor $\widehat{F}$ in the contact term equal to 1 [61]. As becomes clear from Fig. 3.8, the recipe for $\widehat{F}$ suggested by Davidson and Workman gives rise to values in between those produced by the Haberzettl and Ohta form. In several works $[25,41,45]$, it was stressed that $p\left(\gamma, \mathrm{K}^{+}\right) \wedge$ calculations with the Haberzettl or Ohta recipe for $\widehat{F}$ can lead to very different results. So in fact, it comes as no real surprise that a similar remark applies to the recently introduced novel form for $\widehat{F}$.

In Ref. [62], Davidson and Workman studied the effect of hadronic form factors on a multipole analysis of charged pion production. They concluded that the extracted multipoles, for example those listed in Ref. [63], are not heavily affected by 

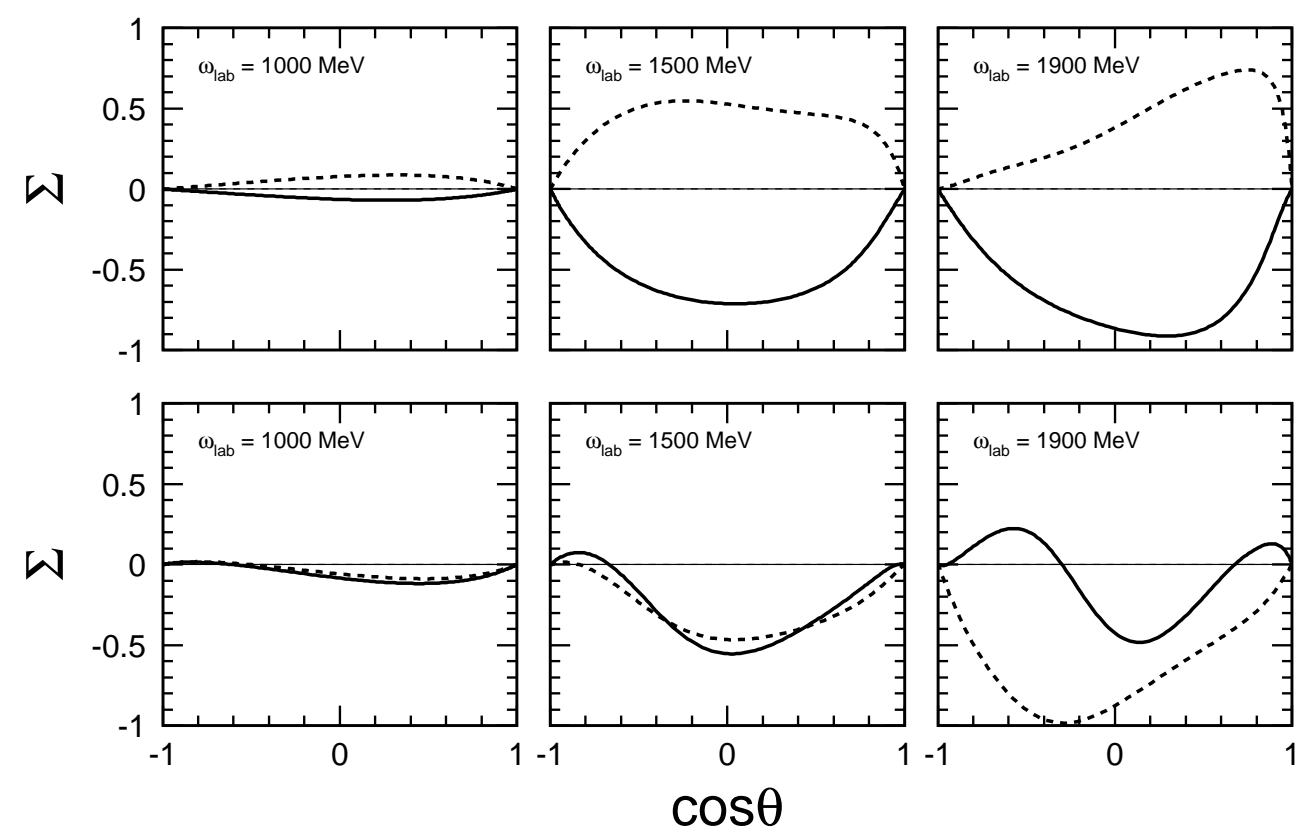

Figure 3.10 The angular distribution of the beam polarization asymmetry for $p\left(\vec{\gamma}, \mathrm{K}^{+}\right) \wedge$ at three photon lab energies. The upper (lower) panels are results with model A (model B) for treating the background diagrams. Solid and dashed lines use the hadronic form factors $\widehat{\mathrm{F}}_{\mathrm{DW}}$ and $\widehat{\mathrm{F}}_{\mathrm{H}}$, respectively.

the form factors. Our calculations indicate that for kaon photoproduction, where the effect of the background terms is larger than in the pion case, great care must be exercised when introducing hadronic form factors and the corresponding gauge restoring contact terms.

We conclude this section with a more general remark. In principle, a correction to a hadronic form factor is not supposed to have a large impact on the reaction dynamics. At best, hadronic form factors are a purely phenomenological tool to polish the (unknown) high energy behavior of the effective field theory. If for some reason, the influence turns out to be large it is obvious that one runs into a rather unsatisfactory situation. In that respect, the introduction of soft hadronic form factors (model A) in modeling the kaon photoproduction process, appears to lead to an unacceptable level of (unphysical) model dependency in the extracted information from fits to $\mathrm{p}\left(\gamma, \mathrm{K}^{+}\right) \wedge$ data. 

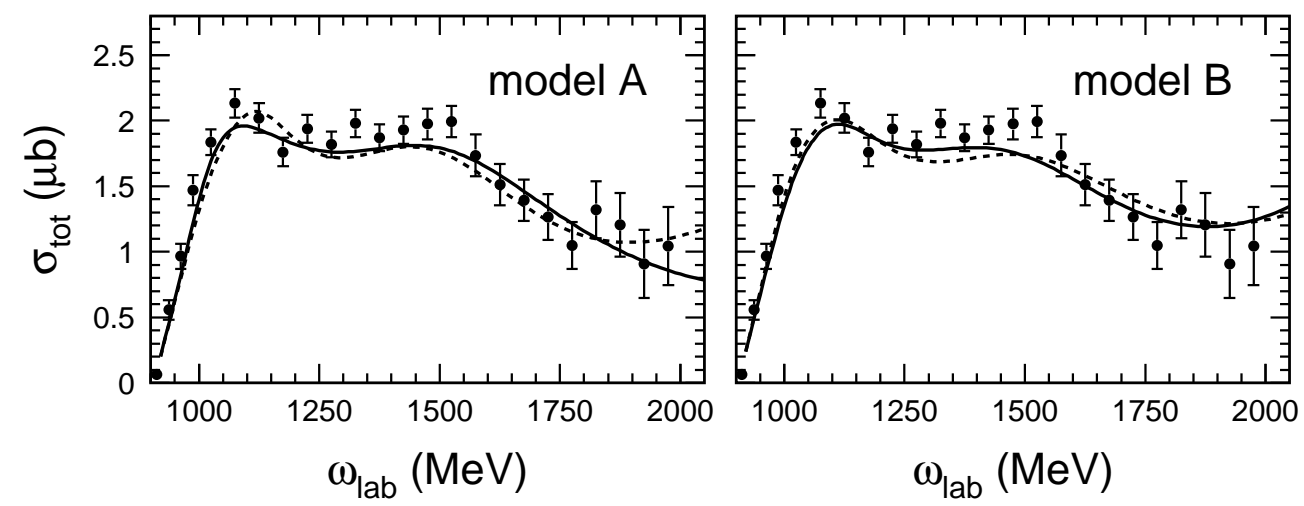

Figure 3.11 Model calculations for the total $p\left(\gamma, K^{+}\right) \wedge$ cross section. The solid curves include the "missing" $\mathrm{D}_{13}$, the dashed lines include a $\mathrm{P}_{13}$ resonance. The left (right) panel uses model A (model B) to describe the background contributions.

\subsubsection{Missing Resonances}

The SAPHIR data [23], released back in 1998, made it clear that the total $\mathrm{p}\left(\gamma, \mathrm{K}^{+}\right) \wedge$ cross section is not characterized by a smooth energy dependence above the threshold peak. The data displayed a structure about photon lab energies of $1.5 \mathrm{GeV}$. Mart and Bennhold [27] interpreted this structure as evidence for an additional resonance and they identified it as a $\mathrm{D}_{13}$ state with a mass of $1895 \mathrm{MeV}$. This $\mathrm{N}^{*}$ state remained unobserved in pion induced and pion photoproduction processes but its existence and appreciable decay in the $\mathrm{K}^{+} \Lambda$ channel was inferred from the constituent quark calculations of Capstick and Roberts [4]. As such, the $\mathrm{D}_{13}(1895)$ appeared as a good candidate for a "missing" resonance. Our calculations, displayed in Fig. 3.11, essentially confirm the observations made in Ref. [27] and reveal that the structure at $\omega_{l a b} \sim 1.5 \mathrm{GeV}$ can be reasonably accounted for after including in the model calculations a $\mathrm{D}_{13}$ resonance in the s-channel. Apart from a $\mathrm{D}_{13}$ state, the quark model calculations of Ref. [4] also predict other $\mathrm{N}^{* \prime}$ s with decay in the strange channels in the mass range about $1.9 \mathrm{GeV}$. Other candidates are $S_{11}(1945)$, $P_{11}$ (1975) and $P_{13}$ (1950). We have performed calculations adding a "missing" $P_{13}$ resonance to the core set of $S_{11}(1650), P_{11}(1710)$ and $P_{13}(1720)$. The results of these model calculations are also contained in Fig. 3.11. It is clear that the procedure of either introducing an extra $\mathrm{D}_{13}$ or a $\mathrm{P}_{13}$ resonance does equally well in reproduc- 
ing the resonant structure in the energy dependence of the total $p\left(\gamma, \mathrm{K}^{+}\right) \wedge$ cross section, independent of the adopted model to handle the background terms. Similar observations were already made in Ref. [27] and there is common agreement on the fact that the reproduction of a visual "bump" in the total cross section should not be interpreted as rock solid evidence for the occurrence of a missing resonance. Nevertheless, in Ref. [27] the $\mathrm{D}_{13}$ was considered to be the preferred candidate on the basis of the agreement between the extracted coupling constants in the fits and the values predicted by the quark model. In the light of the discussions of the model dependences in Secs. 3.2.2 and 3.2.3, we argue that great care must be exercised in drawing conclusions on the basis of the values of the extracted coupling constants. Furthermore, we stress that the calculations of Mart and Bennhold use the Haberzettl recipe for the form factor $\widehat{F}$ and employ a relatively soft cutoff mass $(\Lambda=0.8 \mathrm{GeV})$ for the Born terms. In that respect, their model comes close to what we referred to as model $\mathrm{A}$.

An alternative interpretation of the "bump" in the total cross section of the $p\left(\gamma, K^{+}\right) \wedge$ reaction is put forward by Saghai in Ref. [29]. He pointed out that the structure can be explained in terms of a spin-3/2 $\Lambda^{*}$ resonance in the $u$-channel. We have performed such a calculation that included the $\mathrm{P}_{03}(1890)$ resonance but, as is made clear in Fig. 3.12, we were not able to reveal the observed structure in the total cross section. In this respect, we also argue that a $u$-channel resonance is not likely to produce "structure" in the observables since it never reaches its pole.

In the forthcoming section, a profound study of intermediate resonances for the $\gamma p \rightarrow K \Sigma$ channels will be presented. The existence of a missing $\mathrm{D}_{13}(1895)$ resonance could be put on more solid grounds if it would turn out that the inclusion of this particle also substantially improves the description of the $p\left(\gamma, K^{+}\right) \Sigma^{0}$ and $p\left(\gamma, K^{0}\right) \Sigma^{+}$data.

\subsection{K $\Sigma$ Photoproduction}

In the forthcoming sections, we report our results for the $\mathrm{p}\left(\gamma, \mathrm{K}^{+}\right) \Sigma^{0}$ and $\mathrm{p}\left(\gamma, \mathrm{K}^{0}\right) \Sigma^{+}$ photoproduction reactions. In the discussion, we focus on the both the resonant and background contributions in the reaction amplitude. An important feature which helps in minimizing the number of free parameters, though, is the observation that the $\Sigma^{0}$ and $\Sigma^{+}$particles are part of the $\Sigma$ isospin triplet. Consequently, 


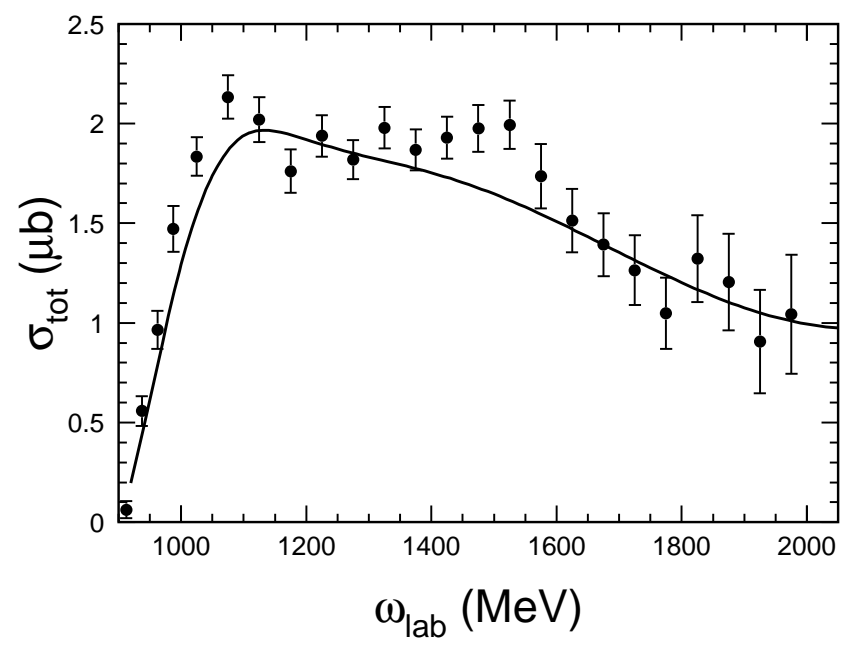

Figure 3.12 Model calculation for the total $p\left(\gamma, \mathrm{K}^{+}\right) \wedge$ cross section. In the s-channel, the nucleon resonances $S_{11}(1650), P_{11}(1710)$ and $P_{13}(1720)$ are taken into account. In the $u$ channel, the $\Lambda^{*}$ resonances $P_{01}(1810)$ and $P_{03}(1890)$ are introduced. The data are from Ref. [23].

one can rely on isospin symmetry to relate the coupling constants needed in the description of the $p\left(\gamma, K^{+}\right) \Sigma^{0}$ reaction to those required for the $p\left(\gamma, K^{0}\right) \Sigma^{+}$process. The technical details of such a procedure are outlined in App. A.2. Within such a scheme, a common analysis of both reaction channels becomes possible. In principle, the $n\left(\gamma, K^{0}\right) \Sigma^{0}$ and $n\left(\gamma, K^{+}\right) \Sigma^{-}$channels could also be implemented in this scheme [12]. Data for those reaction channels are sparse, though. Moreover, the procedure of extracting "elementary" neutron cross sections from measurements on nuclei, like the deuteron, induces severe model dependences. To make matters even worse, connecting proton to neutron electromagnetic coupling constants demands the knowledge of the rather poorly known helicity amplitudes for the different nucleon resonances. For all of the above arguments, we have excluded from our global analyses the $\Sigma$ photoproduction channels off the neutron.

\subsubsection{Resonance Contributions}

Recent isobar models identified the three nucleon resonances $S_{11}(1650), P_{11}(1710)$ and $\mathrm{P}_{13}(1720)$ as leading $\mathrm{N}^{*}$ contributions to the $p\left(\gamma, \mathrm{K}^{+}\right) \Lambda$ reaction $[25,27,29,31]$. It thus appears natural to consider them as privileged candidates to participate in 


\begin{tabular}{|c|c|c|c|c|c|c|c|}
\hline \multicolumn{5}{|c|}{ Resonance Contributions } & \multirow{2}{*}{$\begin{array}{c}\text { Background } \\
\text { Model }\end{array}$} & \multirow[t]{2}{*}{$x^{2}$} & \multirow[t]{2}{*}{ NFP } \\
\hline $\mathrm{N}^{*}$ core set & $\mathrm{D}_{13}(1895)$ & $S_{31}(1620)$ & $S_{31}(1900)$ & $P_{31}(1910)$ & & & \\
\hline 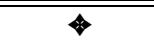 & & & & & $\overline{\mathrm{D}}$ & 6.52 & $\overline{13}$ \\
\hline 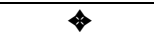 & & 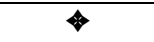 & $\leftarrow$ & & $\mathrm{D}$ & 4.16 & 15 \\
\hline 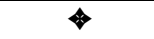 & & 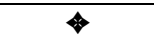 & & $\leftarrow$ & $\mathrm{D}$ & 5.66 & 15 \\
\hline$\downarrow$ & & & 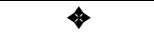 & $\uparrow$ & $\mathrm{D}$ & 3.20 & 15 \\
\hline$\downarrow$ & & 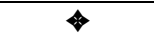 & $\downarrow$ & $\downarrow$ & $\bar{D}$ & 3.19 & 16 \\
\hline$\leftarrow$ & $\leftarrow$ & & & & $\mathrm{D}$ & 5.29 & 18 \\
\hline 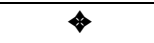 & $\downarrow$ & & $\leftarrow$ & $\leftarrow$ & $\mathrm{D}$ & 2.88 & 20 \\
\hline 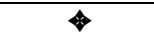 & & & $\downarrow$ & $\uparrow$ & A & 2.03 & 15 \\
\hline$\leftarrow$ & 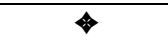 & & $\leftarrow$ & $\leftarrow$ & $\bar{A}$ & 1.98 & 20 \\
\hline$\uparrow$ & & & $\downarrow$ & $\uparrow$ & B & 1.95 & 17 \\
\hline$\leftarrow$ & $\leftarrow$ & & 4 & $\leftarrow$ & B & 1.81 & 22 \\
\hline 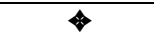 & & & $\leftarrow$ & $\leftarrow$ & C & 1.96 & 15 \\
\hline 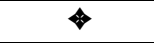 & $\downarrow$ & & $\downarrow$ & 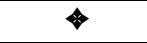 & C & 1.89 & 20 \\
\hline
\end{tabular}

Table 3.3 The table summarizes the $\chi^{2}$ values for the different sets of $\mathrm{N}^{*}$ and $\Delta^{*}$ resonances and a particular model to treat the background diagrams. The $\chi^{2}$ values are from the best fits obtained from comparing the model calculations with the SAPHIR $p(\gamma, K) \Sigma$ data. With "N*core set" we refer to the $\mathrm{S}_{11}(1650), \mathrm{P}_{11}(1710)$ and $\mathrm{P}_{13}(1720)$ nucleon resonances. "NFP" indicates the total number of free parameters in the corresponding fitting procedure.

the $\Sigma$ photoproduction channels [54]. On the other hand, we do not have similar guidelines concerning the leading $\Delta^{*}$ contributions. In our numerical investigations, we rely on a $\chi^{2}$ procedure to judge whether a particular set of resonances is suited to describe the data. In the process of determining an optimum set of $\mathrm{N}^{*}$ and $\Delta^{*}$ particles, we have fixed a basic set consisting of the three aforementioned $\mathrm{N}^{*}$ resonances to which we have gradually added other combinations of $\mathrm{N}^{*}$ and $\Delta^{*}$ states. All results reported in this subsection are obtained with a particular choice (in Sec. 2.3.2 coined model D) for treating the background. A profound discussion of this background implementation is postponed to Sec. 3.3.2.

Starting with a "core set" consisting of the $S_{11}(1650), P_{11}(1710)$ and $P_{13}(1720)$ nucleon resonances, we arrive at $\chi^{2}=6.52$ for an overall fit to the combined set of $\mathrm{p}\left(\gamma, \mathrm{K}^{+}\right) \Sigma^{0}$ and $\mathrm{p}\left(\gamma, \mathrm{K}^{0}\right) \Sigma^{+}$cross section and polarization asymmetry data. This quality of agreement surely allows room for improvement and, consequently, for additional $\mathrm{N}^{*}$ and $\Delta^{*}$ resonances playing a non-negligible role in the reaction dynamics. Table 3.3 summarizes the achieved $\chi^{2}$ values for various combinations of resonances. In an attempt to minimize the number of free parameters, we started 
out with introducing only spin- $1 / 2 \Delta^{*}$ states. Note that in an effective Lagrangian approach, a spin- $1 / 2$ resonance adds only one free parameter while five extra parameters are introduced per spin-3/2 resonance (two coupling constants and three off-shell parameters). Candidates for spin-1/2 $\Delta^{*}$ resonances are the $S_{31}(1620)$, $S_{31}(1900)$ and $P_{31}(1910)$ states [58]. With those three $\Delta^{*}$ resonances and the aforementioned core of three $\mathrm{N}^{*}$ resonances, we arrive at a global best fit with $\chi^{2}=3.19$. So, the introduction of spin-1/2 $\Delta^{*}$ resonances clearly improves the description of the data. A similar quality of agreement $\left(\chi^{2}=3.20\right)$, however, can already be achieved by the mere action of only two of these $\Delta^{* \prime}$, the $S_{31}(1900)$ and $P_{31}(1910)$. Other combinations selected out of the three aforementioned $\Delta^{*}$ states were also able to improve the description of the data (see Table 3.3), although the combination of the $S_{31}(1900)$ and $P_{31}(1910)$ clearly produced the best $\chi^{2}$. Note that these two $\Delta^{*}$ resonances were also recognized as most likely $\mathrm{I}=\frac{3}{2}$ resonance candidates by Mart in his analysis of the $\Sigma$ photoproduction data [54].

In Sec. 3.2, it was put forward that the introduction of a missing $D_{13}(1895)$ resonance is able to enhance the overall agreement of the calculations with the $\mathrm{K}^{+} \wedge$ data. Therefore, we have investigated in how far the inclusion of the $\mathrm{D}_{13}(1895)$ resonance improves the fits of the $K \Sigma$ photoproduction data. Including the core set of three $\mathrm{N}^{* \prime}$ s and the $\mathrm{D}_{13}(1895)$ in the s-channel, we arrive at a best fit with $\chi^{2}=$ 5.29. Inspection of Table 3.3 learns that this quality of agreement is inferior to what was obtained in the calculation with two $\Delta^{*}$ resonances, despite the fact that the $\mathrm{D}_{13}$ and $\Delta^{*}$ resonances have their poles in the same energy region. A resonance set consisting of the core of three $\mathrm{N}^{*}$ resonances, the $\mathrm{D}_{13}$ and the two $\Delta^{* \prime}$ s leads to a fit with $\chi^{2}=2.88$. Compared to the $\chi^{2}$ of 3.20 , achieved without introducing the $D_{13}$, this represents only a minor improvement, in view of the fact that the introduction of a spin-3/2 resonance comes at the expense of throwing in five additional free parameters in the fitting procedure.

Summarizing the findings of Table 3.3, we are tempted to conclude that $\Delta^{*}$ resonances seem to constitute an essential part of the dynamics of $K \Sigma$ photoproduction. No convincing evidence for a salient role for the $D_{13}(1895)$ resonance in $p(\gamma, K) \Sigma$ is found. In this subsection, we have drawn our conclusions on the basis of numerical calculations within one particular model ("model D") for treating the background diagrams. Alternative models for implementing the background diagrams will be 
introduced in the forthcoming section. Anticipating these investigations, the relative role of the different $\mathrm{N}^{*}$ and $\Delta^{*}$ particles turns out to be rather independent of the choices made with respect to the treatment of the non-resonant diagrams. As it happens, this will turn out not always to be the case for the extracted resonance information.

\subsubsection{Background Contributions}

As alluded to in Sec. 2.3.2, one of the long standing issues in modeling strangeness photoproduction is the unrealistically large amounts of strength produced by the "bare" Born terms. In the process of trying to counterbalance the strength from these amplitudes by adding extra ingredients to the theory, it appears that some model dependence in the treatment of the background terms cannot be avoided. We now discuss four models which all succeed in cutting down the background strength in $K \Sigma$ photoproduction. In all schemes, the background contains at least the usual Born terms and the $\mathrm{K}^{*}(892)$ vector meson exchange in the $\mathrm{t}$-channel. For the investigations presented in this section, the resonant part includes the $\mathrm{N}^{*}$ resonances $S_{11}(1650), P_{11}(1710)$ and $P_{13}(1720)$ and the $\Delta^{*}$ states $S_{31}(1900)$ and $P_{31}(1910)$. Those five resonances were identified in Sec. 3.3.1 as an appropriate set for describing $\mathrm{p}(\gamma, \mathrm{K}) \Sigma$ with a minimal number of free parameters.

- Model A: The hadronic form factors $F_{x}(\Lambda)$, described in Eq. (2.2), cut the high momentum dependence of the different amplitudes and emerge as a mechanism to reduce the strength stemming from the Born diagrams to magnitudes of the order of the measured cross sections. To fully exploit the power of this reduction mechanism, we imposed an under limit of $0.4 \mathrm{GeV}$ for the cutoff mass $\Lambda$ during the fit. Thereby, no $Y^{*}$ contributions in the $u$-channel are considered. Despite our reservations regarding the use of soft cutoff masses, eventually we arrive in this scheme at a very satisfactory $\chi^{2}=2.03$ with a cutoff mass $\Lambda$ close to the under limit of $0.4 \mathrm{GeV}$. It should be stressed again that with cutoff masses as small as the kaon mass, the hadronic form factor starts playing a predominant role in the description of the reaction dynamics and heavily affects the predicted values of the observables, not only in the high energy regime but even at threshold. 
- Model B: In Sec. 2.3.2, we pointed out that for the description of $p\left(\gamma, K^{+}\right) \wedge$ processes, the introduction of hyperon resonances in the $u$-channel can be an efficient and physically relevant way of counterbalancing the strength produced by the Born terms. More specifically, we showed in Sec. 3.2.2 that the destructive interference between the $u$-channel amplitudes of the $S_{01}(1800)$ and $\mathrm{P}_{01}(1810)$ hyperon resonances and the Born terms results in a very satisfactory description of the $\mathrm{p}\left(\gamma, \mathrm{K}^{+}\right) \wedge$ data. We have made an attempt to identify an equivalent procedure for $K \Sigma$ photoproduction. Unfortunately, there is relatively little theoretical guidance on how to select the proper intermediate hyperon resonances and how to determine realistic values for their coupling constants. Nevertheless, after including the $\Lambda^{*}(1810)$ and $\Sigma^{*}(1880)$ in the $u$ channel, we arrive at a fair description of the $p\left(\gamma, \mathrm{K}^{+}\right) \Sigma^{0}$ and $\mathrm{p}\left(\gamma, \mathrm{K}^{0}\right) \Sigma^{+}$data with a $\chi^{2}$ of 1.95 .

Note that the $\Lambda^{*}$ resonance does not feed the $\gamma p \rightarrow \mathrm{K}^{0} \Sigma^{+}$channel. Consequently, the procedure of introducing hyperon resonances in the $u$-channel, as a natural physical mechanism to counteract the background amplitudes, is expected to be less effective in the $p\left(\gamma, \mathrm{K}^{0}\right) \Sigma^{+}$channel. However, as can be seen in Fig. 3.13, a stronger destructive interference between the $K^{*}$ vector meson contribution in the $\mathrm{t}$-channel and the Born diagrams is noted for the $\mathrm{p}\left(\gamma, \mathrm{K}^{0}\right) \Sigma^{+}$process. One may wonder why this mechanism does not seem to prevail so strongly in the $\Sigma^{0}$ photoproduction case. This can be naturally explained by looking at the respective electromagnetic coupling constants of the $\mathrm{K}^{*}$ vector mesons. On the basis of Eq. (A.38), one finds that the loss of destructive interference with the $\Lambda^{*}$ resonance in the $p\left(\gamma, \mathrm{K}^{0}\right) \Sigma^{+}$process is likely to be counterbalanced by an enhanced destructive interference with the $t$-channel vector meson exchange.

One of the obvious advantages of the "model B" described here, is that the role of the hadronic form factors can be diminished to levels that appear physically acceptable. Indeed, good fits $\left(\chi^{2}=1.95\right)$ of the $\Sigma$ photoproduction data can be obtained with a hard cutoff mass of the order $\Lambda=1.6 \mathrm{GeV}$. One argument that may speak against model $B$ is that the extracted values of the $\mathrm{Y}^{*}$ coupling constants turn out to be large, compared to the corresponding $\mathrm{N}^{*}$ and $\Delta^{*}$ coupling strengths. However, the two $u$-channel particles, introduced 

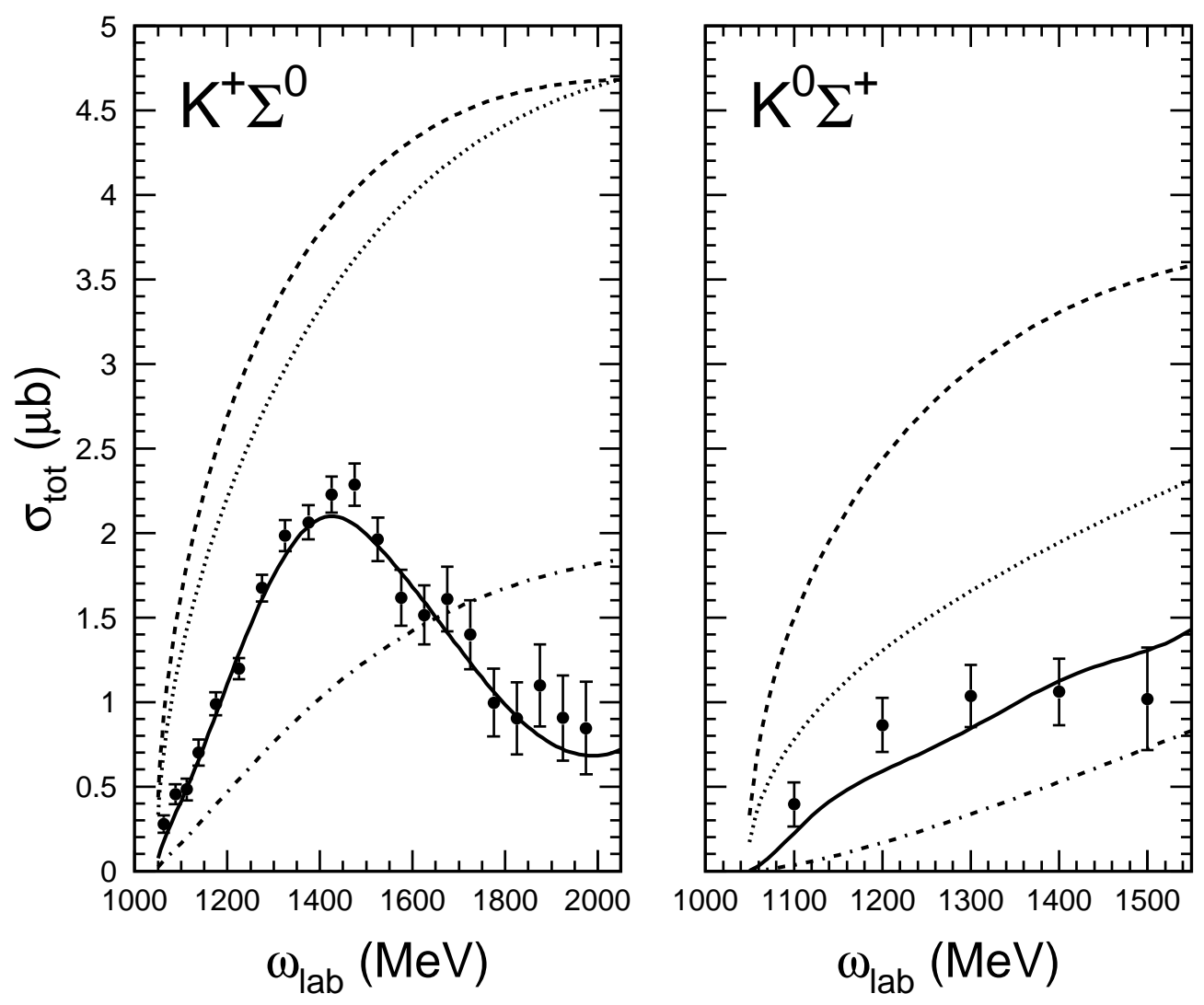

Figure 3.13 Total cross sections for the $p\left(\gamma, \mathrm{K}^{+}\right) \Sigma^{0}$ and $p\left(\gamma, \mathrm{K}^{0}\right) \Sigma^{+}$processes. The dashed curve denotes the computed strength from the Born terms (with hard hadronic form factors, $\Lambda=1.6 \mathrm{GeV}$ ). For the dotted line, the $\mathrm{K}^{*} \mathrm{t}$-channel contribution is added. The dotdashed curve includes the $Y^{*}$ hyperon resonances and consequently is the result of the full background contribution as computed within model B. The solid line embodies, in addition to the background, the s-channel $\mathrm{N}^{*}$ and $\Delta^{*}$ resonances. The data are from Refs. $[23,24]$. 
in the computations, could be interpreted as effective particles for a larger set of $u$-channel processes as outlined in Sec. 3.2.2.

- Model C: A third option is simply to disregard the constraints of Eq. (2.11) imposed by (broken) SU(3)-flavor symmetry. Then, the $g_{K^{+}}{ }_{p}$ and $g_{K^{+} \Sigma^{0} p}$ coupling constants can be treated as free parameters in the minimization procedure. The $\mathrm{p}(\gamma, \mathrm{K}) \Sigma$ results reported by Mart et al. in Ref. [12] are based on such a procedure. In calculations with model $C$ we are solely constraining the relative sign between the two coupling constants. Ignoring $\mathrm{Y}^{*}$ exchange in the $u$-channel, we arrive at an overall agreement with the data of $\chi^{2}=1.96$ with $g_{K+\wedge p} / \sqrt{4 \pi}=-0.23$ and $g_{K+\Sigma 0} / \sqrt{4 \pi}=0.28$. These numbers are dramatically smaller than what is predicted on the basis of SU(3)-flavor symmetry (-3.75 and 1.1, respectively). In this fit, the cutoff mass was allowed to vary freely and adopts a value of $2.5 \mathrm{GeV}$. This value of $\Lambda$ alludes to a rather modest role for the hadronic form factors in the description of the reaction dynamics.

- Model D: This scheme is an attempt to unite some of the virtues of the models $A$ and $B$, at the same time minimizing the number of free parameters that are introduced to compute the background diagrams. In this model, the constraints of Eq. (2.11) are respected during the fitting procedure. In an attempt to keep the model as simple as possible, no $Y^{*}$ particles are introduced. The hadronic cutoff mass $\Lambda$ is treated as a parameter and allowed to vary freely in a range defined by the under limit $1.1 \mathrm{GeV}$. In the optimum fit, the value of $\Lambda$ always approaches this under limit, stressing the essential role of the hadronic form factors for keeping the strength from the Born diagrams at realistic levels. In the previous Sec. 3.3.1, this scheme for treating the background diagrams was adopted when investigating the dominant resonance contributions. Inspecting Table 3.3, it becomes obvious, though, that for a fixed set of resonances, model $D$ systematically leads to $\chi^{2}$ values which are inferior to those obtained in the models A, B and C.

With all suggested models for implementing the background diagrams, we arrive at a satisfactory description of the available data. This feature becomes apparent from the $\chi^{2}$ values contained in Table 3.3 and can also be illustrated by directly comparing model predictions with the data points. A complete list of all numerical 
input parameters for the three models A, B and C are summarized in Table A.3 in App. A.

Fig. 3.14 shows the energy dependence of the total cross section for the background models A, B and C. In these plots, the strength from the background diagrams is also shown. In Figs. 3.15-3.17, model calculations for the angular distribution of the $p\left(\gamma, \mathrm{K}^{+}\right) \Sigma^{0}$ and $\mathrm{p}\left(\gamma, \mathrm{K}^{0}\right) \Sigma^{+}$differential cross section and recoil polarization asymmetry $(P)$ are given. Note, however, that the data points for the asymmetry of the $\mathrm{K}^{0} \Sigma^{+}$process, are binned over the whole experimental energy range. Consequently, they hardly affect the $\chi^{2}$ of the global fit.

Predictions for the energy and angular dependence of the differential cross section and the photon beam asymmetry $(\Sigma)$ are displayed in Figs. 3.18-3.20 for the models A, B and C described above. From visual inspection of the three figures, it indeed becomes apparent that the energy and angular dependence of the differential cross sections is rather similar for the three models. The sudden rise in the predicted $\mathrm{K}^{+} \Sigma^{0}$ cross sections at very backward angles and the highest photon energies should not be considered as physical. It illustrates the limits of the hadronic models for predicting observables in "unmeasured" regions of the phase space. Note that the data used in the fitting procedure do not extend beyond 2.0 $\mathrm{GeV}$ ( $\Sigma^{0}$ production) and $1.55 \mathrm{GeV}\left(\Sigma^{+}\right.$production). For the angular and energy dependence of the $\mathrm{p}\left(\vec{\gamma}, \mathrm{K}^{+}\right) \Sigma^{0}$ photon beam asymmetry, models A, B and C produce comparable results. Although no published data exist for this observable to date, the model dependences in the predictions for this observable seem to be modest. On the other hand, large variations between the different predictions for the $\mathrm{p}\left(\vec{\gamma}, \mathrm{K}^{0}\right) \Sigma^{+}$photon beam asymmetry are observed. To fully appreciate this, we have gathered the calculations for the photon beam asymmetries at some fixed photon lab energies in Fig. 3.21. With no doubt, more precise data for the various polarization observables would help in further constraining the model dependences in the treatment of the background diagrams.

Not only the predictions for some of the asymmetries, but also the extraction of resonance parameters, turns out to be reasonably sensitive to the adopted procedure to treat the background. This feature is illustrated in Fig. 3.22, where the extracted resonance coupling constants are plotted for the background models A, B and $\mathrm{C}$. For the $\mathrm{I}=\frac{3}{2} \Delta^{*}$ resonances, the extracted coupling constants are rather 

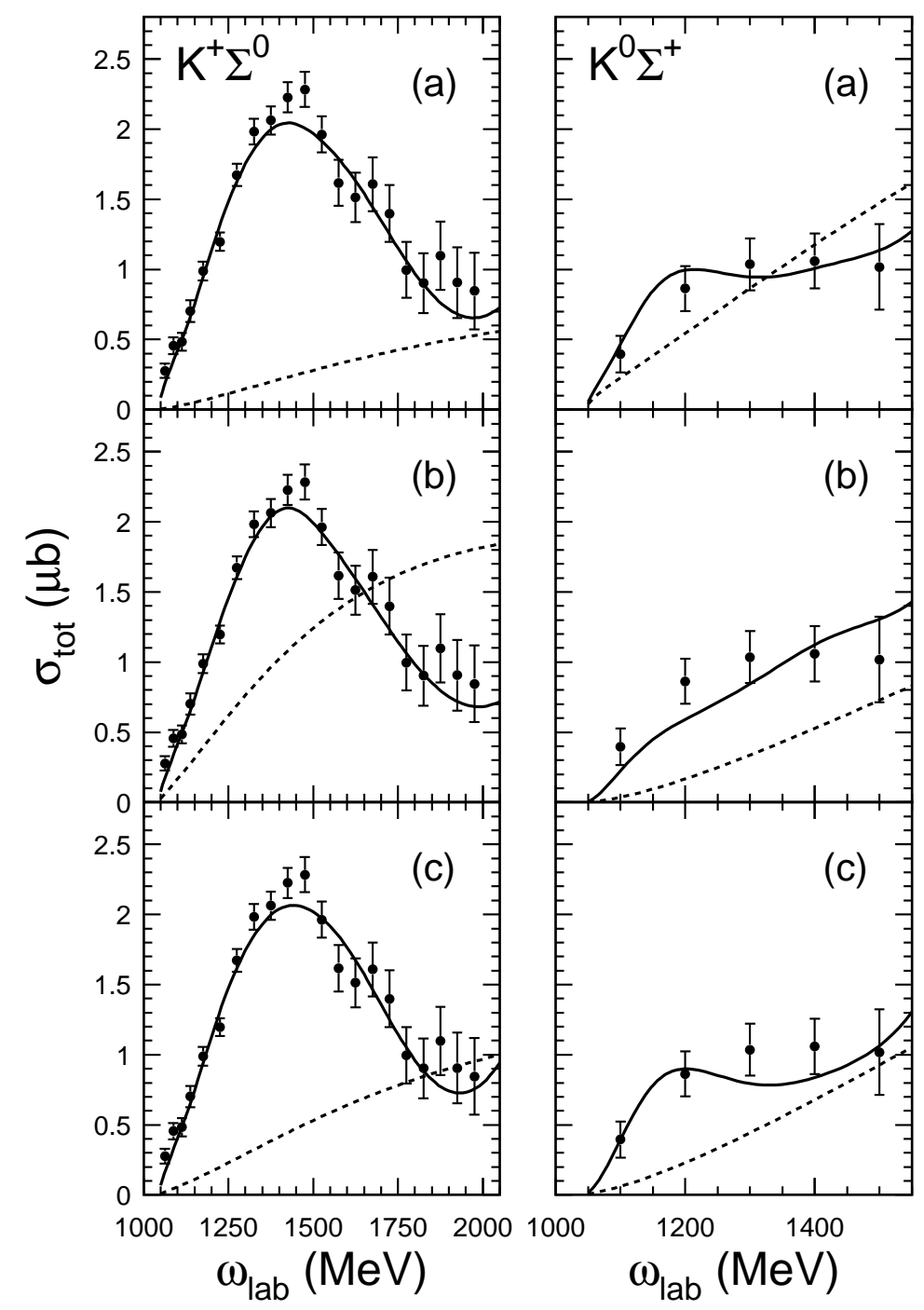

Figure 3.14 Photon energy dependence of the total $p\left(\gamma, \mathrm{K}^{+}\right) \Sigma^{0}$ and $p\left(\gamma, \mathrm{K}^{0}\right) \Sigma^{+}$cross sections. The dashed curves denote the computed strength from the background diagrams. The solid curves include both the background and the resonant amplitudes. The panels (a), (b), (c) refer to the optimum fits obtained with the three background models A, B and C, respectively. The data are from Refs. [23,24]. 


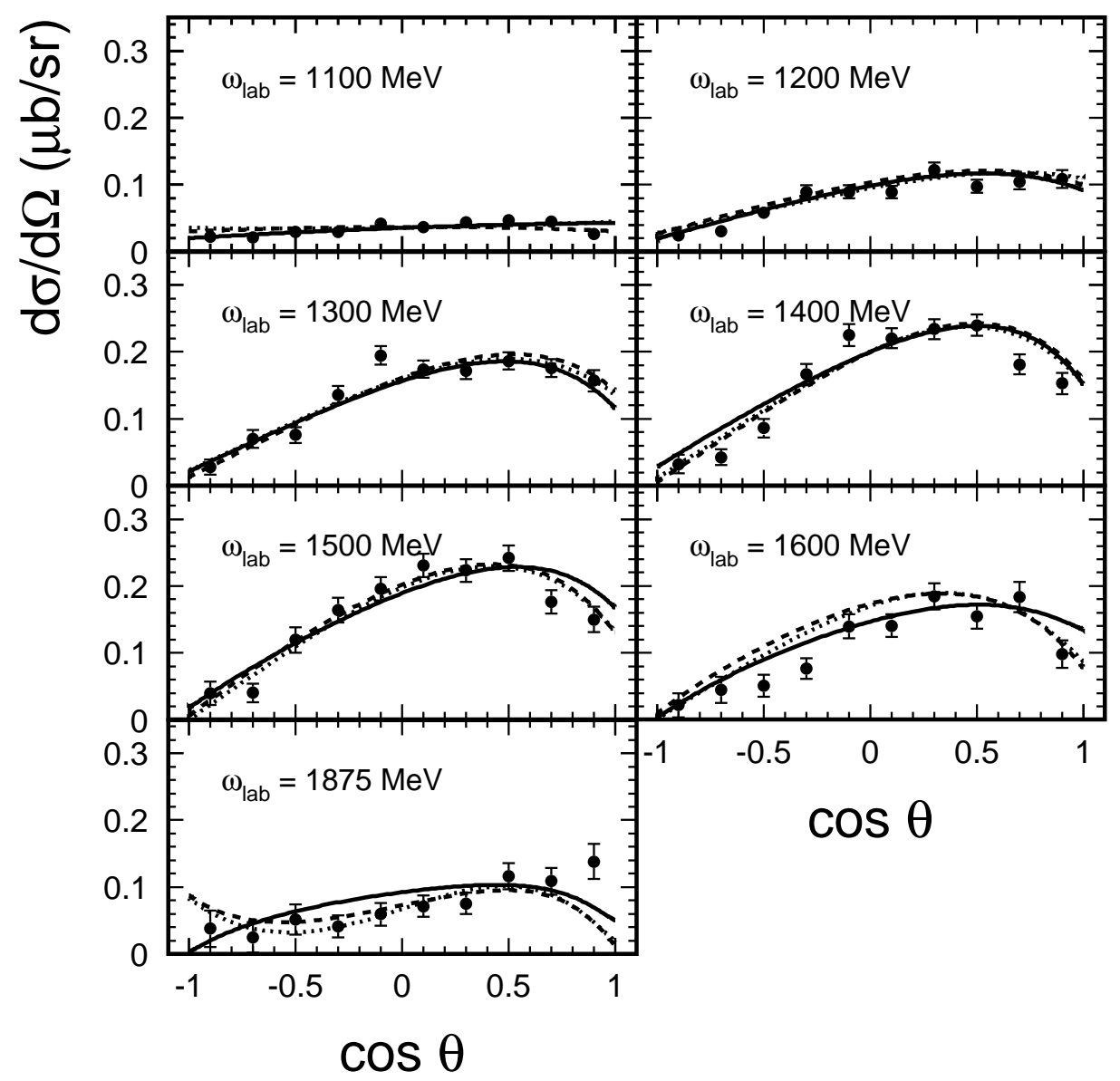

Figure 3.15 Angular dependence of the differential $p\left(\gamma, K^{+}\right) \Sigma^{0}$ cross section for various photon lab energies. The solid, dashed and dotted line are obtained with background model A, B and C, respectively. The data are from Ref. [23]. 


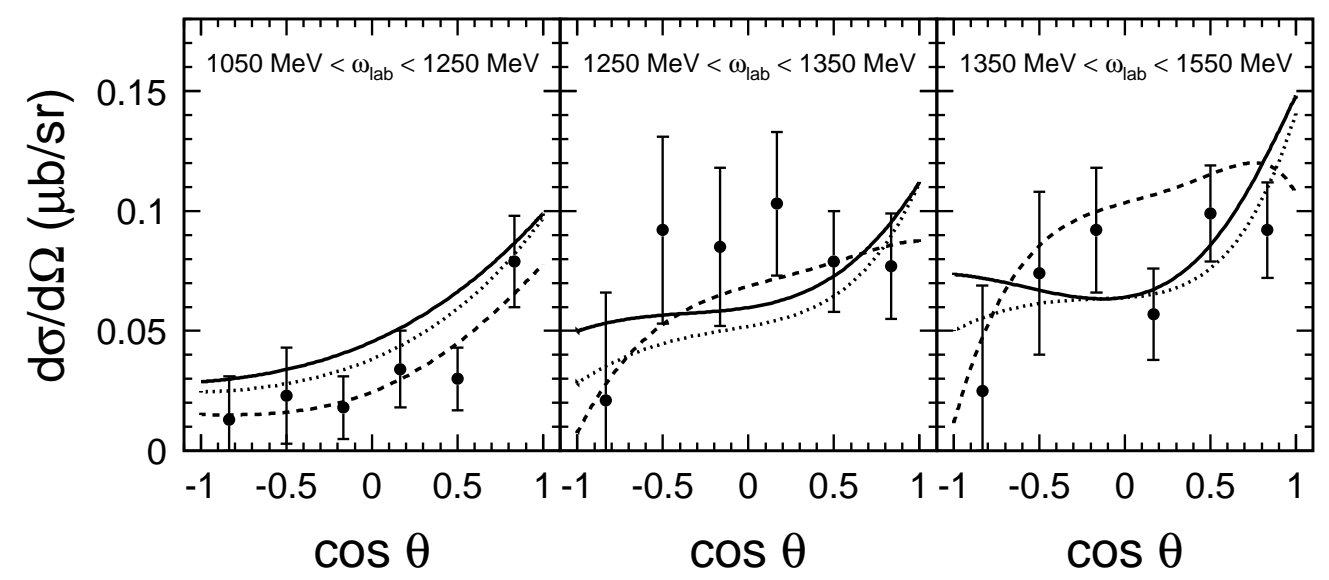

Figure 3.16 Angular dependence of the differential $p\left(\gamma, \mathrm{K}^{0}\right) \Sigma^{+}$cross section for various photon lab energies. Line conventions as in Fig. 3.15. The theoretical curves are averaged over the experimental energy bins. The data are from Ref. [24].
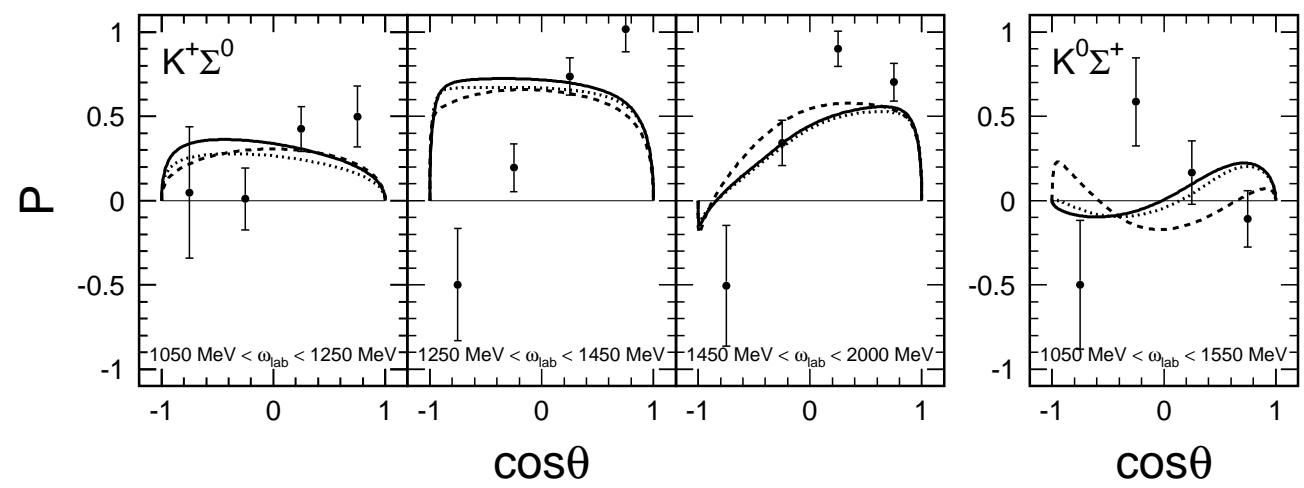

Figure 3.17 Angular dependence of the $p\left(\gamma, \mathrm{K}^{+}\right) \vec{\Sigma}^{0}$ and $p\left(\gamma, \mathrm{K}^{0}\right) \vec{\Sigma}^{+}$recoil polarization asymmetry $(\mathrm{P})$. The line conventions are as in Fig. 3.15. Our results are averaged over the experimental energy bins. The data are from Refs. [23,24]. 

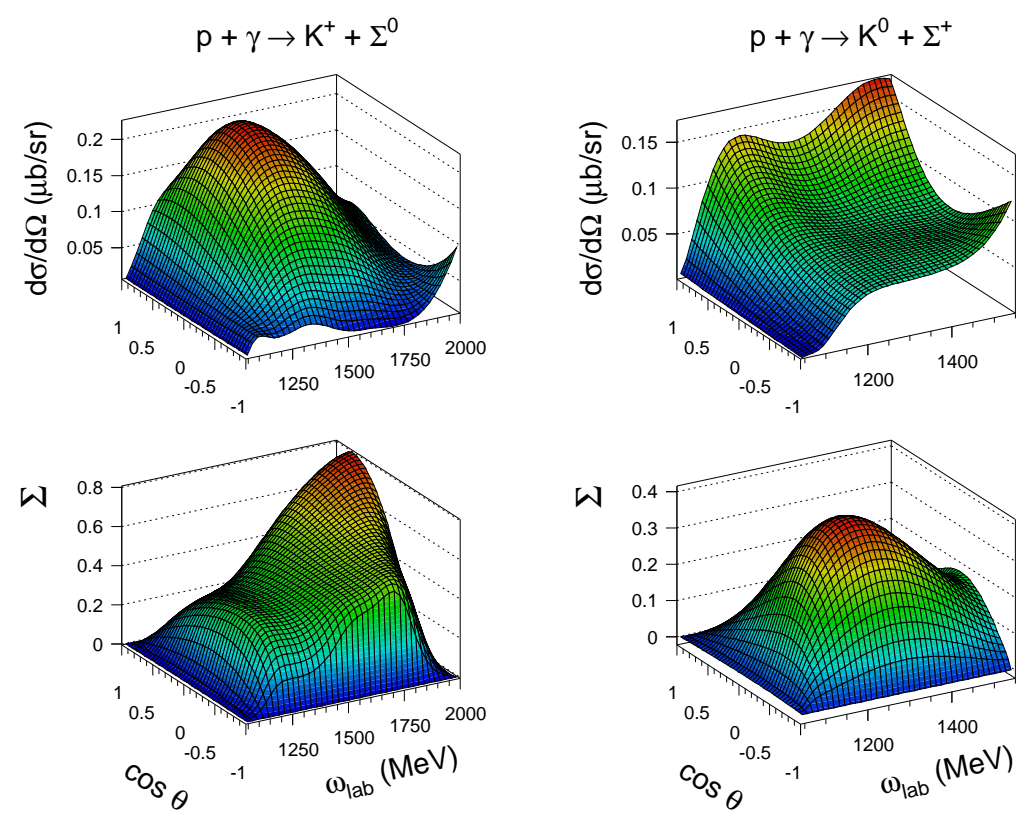

Figure 3.18 The differential cross section and photon beam asymmetry $(\Sigma)$ for the $p\left(\gamma, K^{+}\right) \Sigma^{0}$ and $p\left(\gamma, K^{0}\right) \Sigma^{+}$processes as a function of the photon lab energy $\omega_{\text {lab }}$ and $\cos \theta$. The calculations account for the $S_{11}(1650), P_{11}(1710), P_{13}(1720), S_{31}(1900)$ and $P_{31}(1910)$ $s$-channel resonances. The background terms are treated according to the prescriptions of model A. 

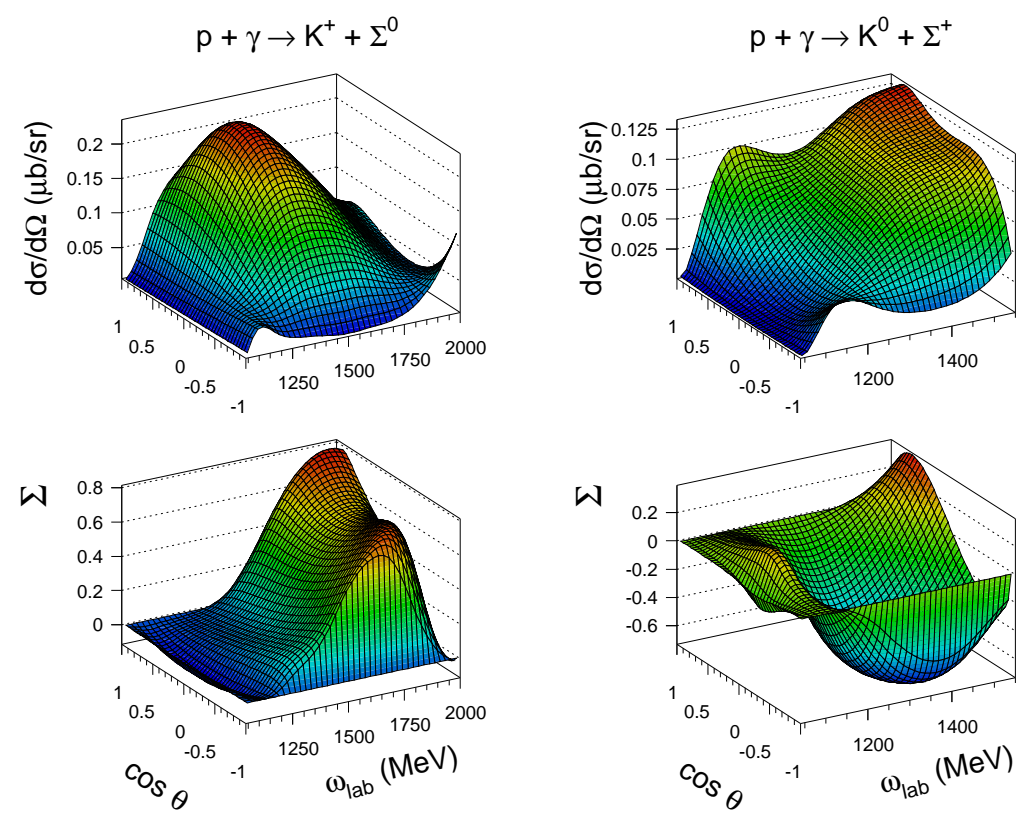

Figure 3.19 As in Figure 3.18 but now for background model B.
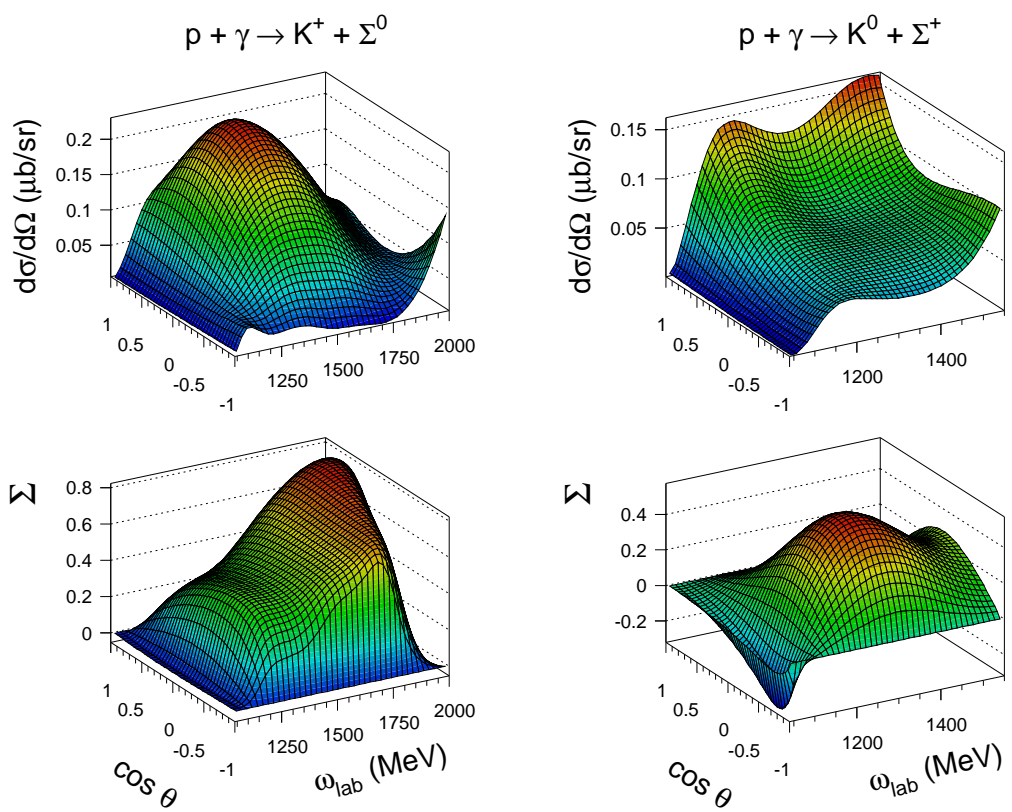

Figure 3.20 As in Figure 3.18 but now for background model C. 

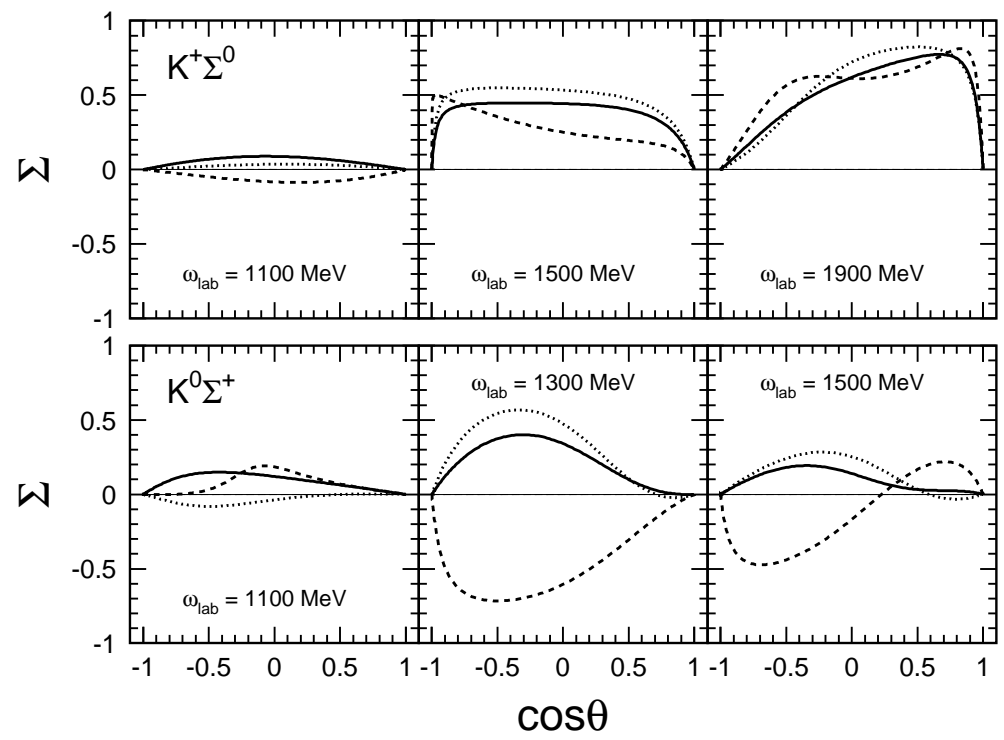

Figure 3.21 The angular dependence of the photon beam asymmetry $(\Sigma)$ for three photon lab energies. The solid, dashed and dotted curves are the predictions as obtained with the background model A, B and C, respectively. The upper panels are for the $p\left(\vec{\gamma}, K^{+}\right) \Sigma^{0}$ process, the lower for the $\mathrm{p}\left(\vec{\gamma}, \mathrm{K}^{0}\right) \Sigma^{+}$reaction.

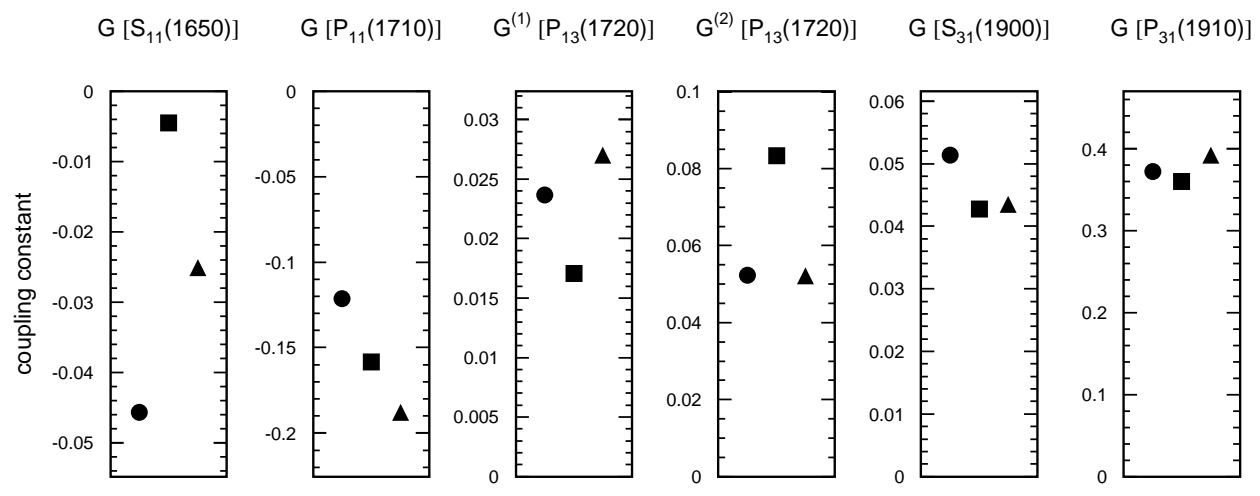

Figure 3.22 The extracted coupling constants for the $\mathrm{N}^{*}$ and $\Delta^{*}$ resonances. The circles are extracted within model A, the squares within model B and the triangles within model C. The conventions adopted for the coupling constants are summarized in App. A. 
insensitive to the choices for the background. Larger variations, exceeding the $20 \%$ level, are observed for the extracted $N^{*}$ parameters. The discerned model dependences in the resonance parameters show that a model independent extraction of this information from the available strangeness photoproduction data is not at hand. This is rather unfortunate, given that these variables play a crucial role in linking the predictions of (constituent) quark models and the photoproduction data.

To conclude this section, we come back to the aforementioned issue of the missing $\mathrm{D}_{13}$ nucleon resonance. Adopting background model $\mathrm{D}$, the inclusion of this $\mathrm{N}^{*}$ particle improved the quality of the global fit from $\chi^{2}=3.20$ to $\chi^{2}=2.88$. We stress again that this comes at the expense of adding five extra parameters. We have investigated whether a similar qualitative feature emerged with background models A, B and C. In all cases, a global fit with the core of the three $\mathrm{N}^{*}$ and the two $\Delta^{*}$ resonances with and without the $\mathrm{D}_{13}(1895)$ was performed. The results are contained in Table 3.3. The improvement in the quality of the fit varied from $3 \%$ (model A) to $8 \%$ (model B). We appreciate this improvement as rather modest in view of the number of extra parameters. For the sake of reference, we mention that in the analysis of the $p\left(\gamma, \mathrm{K}^{+}\right) \wedge$ data in Sec. 3.2, the inclusion of an extra $\mathrm{D}_{13}(1895)$ resonance improved the fits from $22 \%$ up to $40 \%$, depending on the choices made for computing the background contributions. 



\section{Chapter 4}

\section{Strangeness Electroproduction}

In the previous chapter we have presented and discussed the results for the strangeness photoproduction processes. In this chapter, the extension to the electro-induced reactions is made. The modeling of an electroproduction process ep $\rightarrow e^{\prime} \mathrm{KY}$ amounts to describing a virtual photon production reaction of the type $\gamma^{*} p \rightarrow K Y$. Technically, this is achieved in the standard fashion by separating the electron and the hadron current in the electromagnetic interaction Lagrangian. In this procedure, the virtual photon plays the role of intermediate particle that transfers a four momentum $k^{\mu}=(\omega, \vec{k})$ to the hadron current. As a consequence, all effective electromagnetic couplings introduced in the field theory become a function of $\mathrm{Q}^{2} \equiv-\mathrm{k}_{\mu} \mathrm{k}^{\mu}$ for which the limit $\mathrm{Q}^{2} \rightarrow 0$ reduces to the photoproduction case. Immediately, it becomes clear that the scope of the problem to be addressed widens in comparison to the real photon case.

In the literature, only a limited number of theoretical studies on the $p\left(e, e^{\prime} K\right) Y$ reaction can be found $[11,13,38,39,64,65]$. Most of those studies are based on isobar models which try to account for the photo and electroproduction data simultaneously. At present, however, the available $p\left(e, e^{\prime} \mathrm{K}\right) \mathrm{Y}$ data base is too sparse to allow a "dynamical extraction" of a set of $\mathrm{Q}^{2}$-dependent coupling constants. Therefore, our theoretical analysis of the $p\left(e, e^{\prime} \mathrm{K}\right) \mathrm{Y}$ reactions proceeds according to the following major principles. The leading class of diagrams in the reaction dynamics is assumed to be identical for the photo- and electro-induced cases. In a first step, the coupling constants are fixed at values which were determined in the analysis of the photoproduction data reported in the previous chapter. For the $\mathrm{Q}^{2}$-evolution, 
a widely used parameterization is employed for the nucleon electromagnetic form factors and acceptable recipes are adopted at the other electromagnetic vertices. The details of those parameterizations can be found in App. A.3. In what follows, it will become clear that the extrapolation of the photoproduction models into the finite $Q^{2}$ region reveals very interesting features. A refitting procedure of the coupling constants to a combined set of both the photo and electroproduction data will be performed. The overall $\mathrm{Q}^{2}$-dependence of the virtual photon vertices remains fixed by the electromagnetic form factors. In the foreseeable future, the amount of electroproduction data will grow substantially [66] and a dynamical, $\mathrm{Q}^{2}$-dependent extraction of the coupling constants may become feasible.

Before discussing our results for the $p\left(e, e^{\prime} \mathrm{K}\right) \mathrm{Y}$ reactions in Sec. 4.3, in Sec. $4.1 \mathrm{a}$ brief outline is given of the notations and conventions for the observables that are used in this work. The issue of gauge invariance is discussed in Sec. 4.2.

\subsection{Observables for KY Electroproduction}

We now discuss electron scattering processes off the proton with the production of a kaon and a hyperon in the final state:

$$
p(p)+e\left(k_{1}\right) \rightarrow e^{\prime}\left(k_{2}\right)+K\left(p_{K}\right)+Y\left(p_{Y}\right)
$$

The corresponding four momenta are defined as:

$$
\begin{aligned}
& k_{1}^{\mu}=\left(\epsilon_{1}, \vec{k}_{1}\right), \quad k_{2}^{\mu}=\left(\epsilon_{2}, \vec{k}_{2}\right), \quad k^{\mu}=(\omega, \vec{k}) \text {, } \\
& p^{\mu}=\left(E_{p},-\vec{k}^{*}\right), \quad p_{k}^{\mu}=\left(E_{k}, \vec{p}_{K}\right), \quad p_{Y}^{\mu}=\left(E_{Y},-\vec{p}_{K}\right) \text {, }
\end{aligned}
$$

with $k^{\mu}=k_{1}^{\mu}-k_{2}^{\mu}$ the four momentum of the virtual photon in the lab frame. To construct the cross section, it is a common procedure to describe the electron kinematics in the lab frame and the hadron physics in the center-of-mass (c.m.) frame of the proton target and the virtual photon. This is schematically depicted in Fig. 4.1. Accordingly, the hadron four momenta are evaluated in the c.m. frame. The virtual photon four vector establishes the relation between the kinematical variables in the two frames. Its four momentum will be denoted as $k^{* \mu}=\left(\omega^{*}, \vec{k}^{*}\right)$ when it is expressed in the c.m. frame. The Lorentz boost factors connecting those quantities 


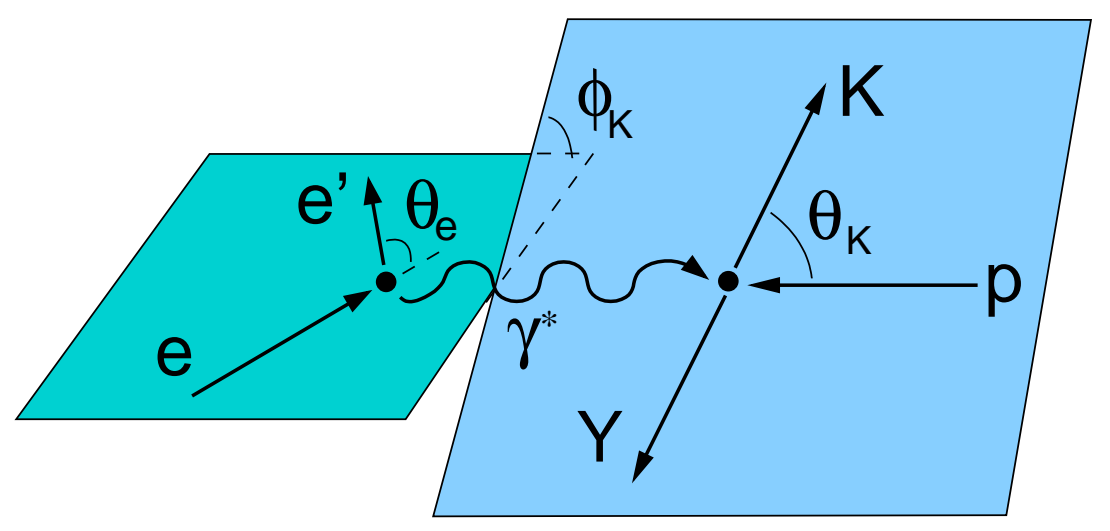

Figure 4.1 Orientation of the reference frames and definition of the kinematic quantities for the $p\left(e, e^{\prime} \mathrm{K}\right) \mathrm{Y}$ electroproduction process.

are:

$$
\begin{aligned}
\vec{k}^{*} & =\vec{k}\left(\frac{m_{p}}{W}\right) \\
\omega^{*} & =W-\left(\omega+m_{p}\right) \frac{m_{p}}{W} \\
& =\frac{s-m_{p}^{2}-Q^{2}}{2 W} .
\end{aligned}
$$

Herein, $W \equiv \sqrt{s}$ is the total c.m. energy and $Q^{2}=-k_{\mu} k^{\mu}$. Then, the electroproduction cross section can be written as:

$$
\frac{\mathrm{d} \sigma}{\mathrm{d} \epsilon_{2} \mathrm{~d} \Omega_{2} \mathrm{~d} \Omega_{\mathrm{K}}}=\frac{1}{32(2 \pi)^{5}} \frac{1}{\mathrm{~m}_{\mathrm{p}}} \frac{\left|\vec{p}_{\mathrm{K}}\right|}{W} \frac{\epsilon_{2}}{\epsilon_{1}} \sum_{\lambda^{\prime} \mathrm{s}}\left|M_{\lambda^{\prime} \mathrm{s}}\right|^{2},
$$

where $M_{\lambda^{\prime} s}$ now depends on the incoming and outgoing electron polarization and the nucleon and hyperon spin state. The structure of the virtual photon, which connects the electron to the hadron current, allows a separation of the amplitude in terms of longitudinal and transverse parts. For unpolarized electron scattering, one gets:

$$
\begin{aligned}
& \frac{d \sigma}{d \epsilon_{2} d \Omega_{2} d \Omega_{K}}= \\
& \Gamma\left[\frac{d \sigma_{T}}{d \Omega_{K}}+\epsilon \frac{d \sigma_{L}}{d \Omega_{K}}+\epsilon \frac{d \sigma_{T}}{d \Omega_{K}} \cos \left(2 \phi_{K}\right)+\sqrt{\epsilon(1+\epsilon)} \frac{d \sigma_{T L}}{d \Omega_{K}} \cos \left(\phi_{K}\right)\right] .
\end{aligned}
$$

Herein, the virtual photon flux factor is given by:

$$
\Gamma=\frac{\alpha}{2 \pi^{2}} \frac{\epsilon_{2}}{\epsilon_{1}} \frac{K_{H}}{Q^{2}} \frac{1}{1-\epsilon},
$$


with $\mathrm{K}_{\mathrm{H}}=\omega-\mathrm{Q}^{2} /\left(2 \mathrm{~m}_{\mathrm{p}}\right)$ the equivalent real photon lab energy. Further, $\epsilon$ is the degree of transverse polarization of the virtual photon:

$$
\epsilon=\left(1+\frac{2|\vec{k}|^{2}}{\mathrm{Q}^{2}} \tan ^{2} \frac{\theta_{e}}{2}\right)^{-1} .
$$

Note that the $\phi_{K}$-dependence is extracted from the various structure functions. As a consequence, they solely depend on the variable set $\left(\omega^{*},\left|\vec{k}^{*}\right|, \theta_{K}\right)$ or equivalently $\left(s, t, Q^{2}\right)$. The various contributions to the virtual photon cross sections are defined as:

$$
\begin{aligned}
\frac{\mathrm{d} \sigma_{\mathrm{T}}}{\mathrm{d} \Omega_{\mathrm{K}}} & =\chi \frac{1}{(4 \pi)^{2}}\left(\mathcal{H}_{1,1}+\mathcal{H}_{-1,-1}\right), \\
\frac{\mathrm{d} \sigma_{\mathrm{L}}}{\mathrm{d} \Omega_{\mathrm{K}}} & =2 \chi \frac{1}{(4 \pi)^{2}} \mathcal{H}_{0,0}, \\
\frac{\mathrm{d} \sigma_{\mathrm{T}}}{\mathrm{d} \Omega_{\mathrm{K}}} & =-\chi \frac{1}{(4 \pi)^{2}}\left(\mathcal{H}_{1,-1}+\mathcal{H}_{-1,1}\right), \\
\frac{\mathrm{d} \sigma_{\mathrm{TL}}}{\mathrm{d} \Omega_{\mathrm{K}}} & =-\chi \frac{1}{(4 \pi)^{2}}\left(\mathcal{H}_{0,1}+\mathcal{H}_{1,0}-\mathcal{H}_{-1,0}-\mathcal{H}_{0,-1}\right) .
\end{aligned}
$$

The factor $\chi$ is given by:

$$
x \equiv \frac{1}{16} \frac{1}{W^{2}} \frac{\left|\vec{p}_{K}\right|}{K_{H}} \frac{W}{m_{p}} .
$$

The hadronic tensors occurring in the Eqs. (4.9)-(4.12) can be expressed in terms of traces:

$$
\begin{aligned}
\mathcal{H}_{\lambda \lambda^{\prime}} & =\sum_{\lambda_{i} \lambda_{f}} \mathcal{M}_{\lambda}^{\lambda_{i} \lambda_{f}}\left(M_{\lambda^{\prime}}^{\lambda_{i} \lambda_{f}}\right)^{\dagger}, \\
& =\sum_{\lambda_{i} \lambda_{f}}\left(\bar{u}_{Y}^{\lambda_{f}}\left(p_{Y}\right) T^{\mu} \varepsilon_{\mu}^{\lambda} u_{p}^{\lambda_{i}}(p)\right)\left(\bar{u}_{p}^{\lambda_{i}}(p) \bar{T}^{v} \varepsilon_{v}^{\lambda^{\prime *}} u_{Y}^{\lambda_{f}}\left(p_{Y}\right)\right), \\
& =\operatorname{Tr}\left\{\left(\not p_{Y}+m_{Y}\right) T^{\mu} \varepsilon_{\mu}^{\lambda}\left(\not p+m_{p}\right) \bar{T}^{v} \varepsilon_{v}^{\lambda^{\prime} *}\right\},
\end{aligned}
$$

where $\lambda$ and $\lambda^{\prime}$ denotes the polarization of the virtual photon. The virtual photon polarization vectors are defined as:

$$
\varepsilon^{\lambda=0}=\frac{1}{\sqrt{Q^{2}}}\left(\left|\vec{k}^{*}\right|, 0,0, \omega^{*}\right), \quad \varepsilon^{\lambda= \pm 1}=\mp \frac{1}{\sqrt{2}}(0,1, \pm i, 0) .
$$

Remark that $\varepsilon^{\lambda=0}$ has to be evaluated with the virtual photon energy and momentum determined in the frame in which the hadronic tensor is computed. 
So far, the expressions for electroproduction were derived for unpolarized particles both in the initial as well as in the final state. When polarized electrons are used in the experiment, two additional terms enter the expression for the cross section:

$$
\begin{aligned}
\frac{\mathrm{d} \sigma}{\mathrm{d} \epsilon_{2} \mathrm{~d} \Omega_{2} \mathrm{~d} \Omega_{\mathrm{K}}}= & \left.\frac{\mathrm{d} \sigma}{\mathrm{d} \epsilon_{2} \mathrm{~d} \Omega_{2} \mathrm{~d} \Omega_{\mathrm{K}}}\right|_{\text {unpol }}+ \\
& \mathrm{h} \Gamma\left[\sqrt{1-\epsilon^{2}} \frac{\mathrm{d} \sigma_{\Pi^{\prime}}}{\mathrm{d} \Omega_{\mathrm{K}}}+\sqrt{\epsilon(\epsilon-1)} \frac{\mathrm{d} \sigma_{\mathrm{TL}^{\prime}}}{\mathrm{d} \Omega_{\mathrm{K}}} \sin \left(\phi_{\mathrm{K}}\right)\right] .
\end{aligned}
$$

Herein, $h$ is the helicity of the incident electron. Further, the two additional virtual photon cross sections are defined as:

$$
\begin{aligned}
\frac{\mathrm{d} \sigma_{\Pi^{\prime}}}{\mathrm{d} \Omega_{\mathrm{K}}} & =-\chi \frac{1}{(4 \pi)^{2}}\left(\mathcal{H}_{1,1}-\mathcal{H}_{-1,-1}\right) \\
\frac{\mathrm{d} \sigma_{\mathrm{TL}^{\prime}}}{\mathrm{d} \Omega_{\mathrm{K}}} & =\chi \frac{1}{(4 \pi)^{2}}\left(\mathcal{H}_{0,1}+\mathcal{H}_{1,0}+\mathcal{H}_{-1,0}+\mathcal{H}_{0,-1}\right),
\end{aligned}
$$

where the hadronic tensors are as in Eq. (4.14). Because of the structure of the hadron current, the $\pi^{\prime}$ term is identical to zero if no baryon polarizations are involved in the process. In case one of the baryons (the $p$ or $Y$ ) becomes polarized, the same technique as in the real photon case can be applied: insert an additional spin projection operator $\Pi^{ \pm}(n)=\frac{1}{2}\left(1 \pm \gamma_{5} \not h\right)$ in the trace calculation. The polarized cross section can again be expressed in terms of the various response functions. More details can be found in e.g. Ref. [67].

\subsection{Gauge Invariance}

Gauge invariance is a fundamental symmetry of every theory dealing with electromagnetic interactions. We will present the discussion for the $p\left(e, e^{\prime} K^{+}\right) \wedge$ reaction, but extension to the other isospin channels is straightforward. In order to impose gauge invariance, the total amplitude has to fulfill the Lorentz condition:

$$
k_{\mu} M^{\mu}=0
$$

In the effective Lagrangian approach, all terms naturally obey this constraint individually, apart from those Born terms that contain an "electric coupling". As outlined in Sec. 2.2, it is only the sum of all the amplitudes containing an electric 
couping which fulfill the Lorentz condition. With the introduction of electromagnetic form factors, however, the individual electric parts are modified by different functions $F_{1}^{p}\left(Q^{2}\right)$ and $F_{K}\left(Q^{2}\right)$ :

$$
\begin{aligned}
\mathcal{L}_{\gamma^{*} p p} & =-e F_{1}^{p}\left(Q^{2}\right) \bar{N} \gamma_{\mu} N A^{\mu}+\frac{e K_{p}}{4 m_{p}} F_{2}^{p}\left(Q^{2}\right) \bar{N} \sigma_{\mu \nu} N F^{\mu \nu}, \\
\mathcal{L}_{\gamma^{*} K K} & =-i e F_{K}\left(Q^{2}\right)\left(K^{\dagger} \partial_{\mu} K-K \partial_{\mu} K^{\dagger}\right) A^{\mu}, \\
\mathcal{L}_{\gamma^{*} \Lambda \Lambda} & =-e F_{1}^{\wedge}\left(Q^{2}\right) \bar{\Lambda} \gamma_{\mu} \wedge A^{\mu}+\frac{e K_{\Lambda}}{4 m_{p}} F_{2}^{\wedge}\left(Q^{2}\right) \bar{\Lambda} \sigma_{\mu \nu} \wedge F^{\mu \nu} .
\end{aligned}
$$

In addition, the $\gamma^{*} \Lambda \Lambda$ interaction Lagrangian of Eq. (4.22) receives and additional electric $\gamma^{\mu}$ coupling proportional to $\mathrm{F}_{1}^{\wedge}\left(\mathrm{Q}^{2}\right)$. This contribution is comparable to the $\mathrm{Q}^{2}$-dependent electric part of the neutron, which is observed to be small, though. It is obvious that after those modifications, the gauge invariance of the combined electric terms is lost.

To restore current conservation, we follow the procedure of Gross and Riska [68], based on the Ward-Takahashi identity. They suggest the following replacements in the vertex functions:

$$
\begin{aligned}
\mathrm{F}_{1}^{\mathrm{p}, \wedge}\left(\mathrm{Q}^{2}\right) \gamma_{\mu} & \longrightarrow \mathrm{F}_{1}^{\mathrm{p}, \wedge}\left(\mathrm{Q}^{2}\right)\left[\gamma_{\mu}+\frac{k}{\mathrm{Q}^{2}} \mathrm{k}_{\mu}\right]-\mathrm{F}_{1}^{\mathrm{p}, \wedge}(0) \frac{k}{\mathrm{Q}^{2}} \mathrm{k}_{\mu} \\
\mathrm{F}_{\mathrm{K}}\left(\mathrm{Q}^{2}\right)\left(2 \mathrm{p}_{\mathrm{K}}-\mathrm{k}\right)_{\mu} \longrightarrow & \mathrm{F}_{\mathrm{K}}\left(\mathrm{Q}^{2}\right)\left[\left(2 \mathrm{p}_{\mathrm{K}}-\mathrm{k}\right)_{\mu}+\frac{\left(2 \mathrm{p}_{\mathrm{K}}-\mathrm{k}\right) \cdot \mathrm{k}}{\mathrm{Q}^{2}} \mathrm{k}_{\mu}\right] \\
& -\mathrm{F}_{\mathrm{K}}(0)\left[\frac{\left(2 \mathrm{p}_{\mathrm{K}}-\mathrm{k}\right) \cdot \mathrm{k}}{\mathrm{Q}^{2}} \mathrm{k}_{\mu}\right] .
\end{aligned}
$$

With those replacements, it is straightforward to see that the Lorentz condition of Eq. (4.19) is fulfilled at any $\mathrm{Q}^{2}$ provided that the total amplitude in the real photon limit $\left(Q^{2} \rightarrow 0\right)$ respects gauge invariance. In other words, if the effective field theory conserves the current for the real photon process, the procedure of Gross and Riska allows to construct a gauge invariant theory for the virtual photon process. It is important to remark that the extra terms, introduced in Eqs. (4.23) and (4.24) in order to ensure the gauge invariance, do not contribute to the observables. Since all the additional terms in Eqs. (4.23) and (4.24) are proportional to $\mathrm{k}^{\mu}$, they effectively vanish in the calculations of the observables since they are orthogonal to the virtual polarization vector $\varepsilon_{\mu}(k \cdot \varepsilon=0)$. In Ref. [69], Nozawa and Lee showed 
that this procedure can be related to the vector meson dominance model for the electromagnetic form factors.

\subsection{Results and Discussion}

To date, very few $p\left(e, e^{\prime} \mathrm{K}\right) \mathrm{Y}$ data are available. With no doubt, the amount of data is too small to perform a reliable extraction of $\mathrm{Q}^{2}$-dependent coupling constants. However, it is interesting to confront the models constructed for real photon production processes with the available electroproduction data. In this section, the $\mathrm{K}^{+} \Lambda$ and $\mathrm{K} \Sigma$ reactions are treated separately.

\subsubsection{K^ Electroproduction}

We start from the three models which have been developed in Sec. 3.2 to describe real photon induced $\mathrm{K}^{+} \Lambda$ production. In the extrapolation of the calculations into the finite $\mathrm{Q}^{2}$ region, the sets of coupling constants are taken from the real photon cases and in order to account for the $\mathrm{Q}^{2}$-dependence, electromagnetic form factors are inserted as outlined in App. A.3. The results of those calculations are presented in Fig. 4.2, where the $Q^{2}$-dependence of the $d \sigma_{L} / d \Omega$ and $d \sigma_{T} / d \Omega$ term is plotted.

Let us first discuss the transverse component $d \sigma_{T} / d \Omega$. In the limit $Q^{2} \rightarrow 0$, all three models are constrained by the real photon $K^{+} \wedge$ data. At finite $Q^{2}$, however, the model calculations fall off more rapidly than the data. This may indicate that the $\mathrm{Q}^{2}$-dependence of the resonance coupling constants, here reflecting itself as the nucleon Pauli form factor, is not appropriate. Anticipating this shortcoming, we have performed calculations with a simple dipole form factor at the electromagnetic vertex of the resonances. By variation of the dipole cutoff mass, we were able to raise the strength for this transverse response function. However, by comparing the JLab data point at $\mathrm{Q}^{2}=0.5 \mathrm{GeV}^{2}$ and the corresponding SAPHIR point of about $0.3 \mu \mathrm{b} / \mathrm{sr}$ for the same kinematics but $\mathrm{Q}^{2}=0$, a very smooth fall off or even a slow rise in the low $\mathrm{Q}^{2}$ region seems to emerge. So far, all of our predictions for the transverse $p\left(e, e^{\prime} K^{+}\right) \wedge$ response fall off with increasing $Q^{2}$ and we are not able to reproduce this effect.

For the longitudinal cross section $d \sigma_{\mathrm{L}} / \mathrm{d} \Omega$, the situation is completely different. Since the longitudinal component of the amplitude is not "probed" by real photons, 

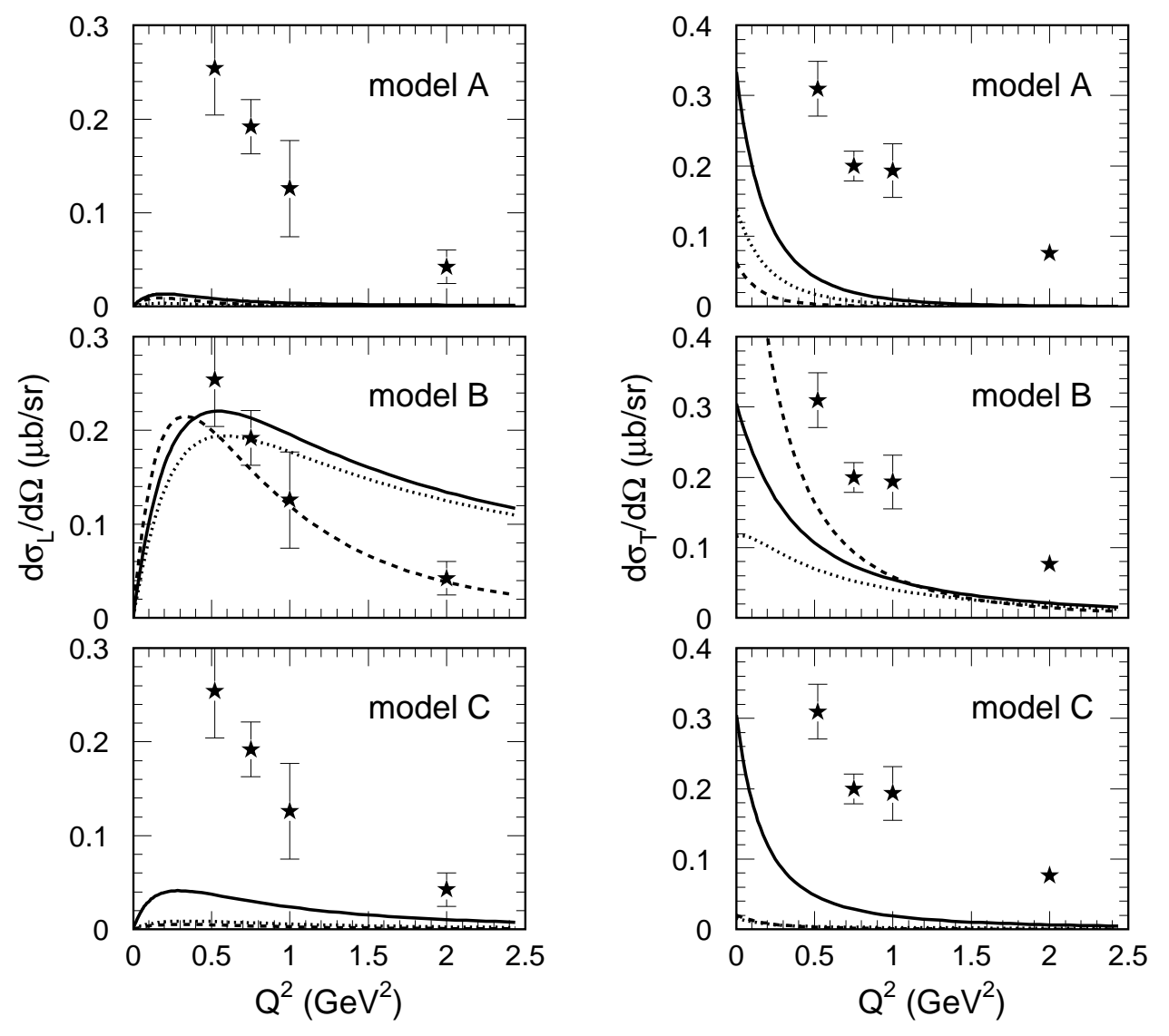

Figure 4.2 $Q^{2}$-dependence of the $d \sigma_{L} / d \Omega$ (left) and $d \sigma_{T} / d \Omega$ (right) terms for the $p\left(e, e^{\prime} K^{+}\right) \wedge$ process at $W=1.84 \mathrm{GeV}$ and $\cos \theta=1$. The solid curve is the full calculation. The dashed line is the strength produced by the Born terms with hadronic form factors included. The dotted line represents the contributions from all background diagrams. The panels refer to the three different models ( $\mathrm{A}, \mathrm{B}$ and $\mathrm{C}$ ) which have been introduced to implement the contributions from the background. The data are from Ref. [70]. 
this part remains absent in the real photon observables. As a consequence, the longitudinal part of the amplitude is not constrained by the real photon data. In this light, this response function can serve as a good test for the predictive power of the models and their corresponding parameters. From Fig. 4.2 it becomes obvious that the models $\mathrm{A}$ and $\mathrm{C}$ badly fail in describing the longitudinal part of the electroproduction data. Only with model B, one obtains the correct order of magnitude for this term. This feature illustrates the importance of the background diagrams in the longitudinal response. In order to trace the origin for the dramatic failure of model A and C, we have plotted the contributions of the Born terms as dashed lines in this Fig. 4.2. It is important to note that this curve does not represent the point-like Born terms. They are modified by the hadronic form factors. In model A, a soft form factor is applied with a cutoff mass of $\Lambda=0.4 \mathrm{GeV}$. On the contrary, for model $B$, the Born terms are weighed with a hard hadronic form factor $(\Lambda=1.5$ $\mathrm{GeV})$. It is clear that when adopting a soft form factor, the longitudinal component of the Born terms is too strongly suppressed. A similar remark appears to model $\mathrm{C}$ where a moderate cutoff mass $(\Lambda=1.85 \mathrm{GeV})$ is introduced, but where small values for the coupling constants $g_{K^{+}} \Lambda_{p}$ and $g_{K^{+} \Sigma^{0}}$, out of the SU(3)-flavor symmetry ranges, are adopted. Fig. 4.2 also shows the total background strength. For models $\mathrm{A}$ and $\mathrm{C}$, the background consists of the Born terms and the $\mathrm{t}$-channel $\mathrm{K}^{*}$ and $K_{1}$ exchange terms. For model $B$, the Born terms are extended with the same two t-channel terms and two hyperon exchanges in the $u$-channel. Note that the full background strength for the longitudinal response differs not that much from the strength produced by the Born terms alone. This indicates that the contribution from the t-channel exchanges to the longitudinal response is small. The most significant deviation exists for model B. The difference here can be attributed mostly to the hyperon resonances which are included in the $u$-channel.

The same tendencies with respect to the predictions of model A, B and C are observed when the model calculations are compared to the data for the $\phi_{\mathrm{K}}$-averaged virtual photon differential cross section for which only two terms survive:

$$
\frac{\mathrm{d} \sigma_{\mathrm{T}}}{\mathrm{d} \Omega_{\mathrm{K}}}+\epsilon \frac{\mathrm{d} \sigma_{\mathrm{L}}}{\mathrm{d} \Omega_{\mathrm{K}}}
$$

The model calculations are plotted in Fig. 4.3 against the available data from Cornell $[71,72]$ and the CEA facility at Harvard [73]. From this figure, it is obvious 


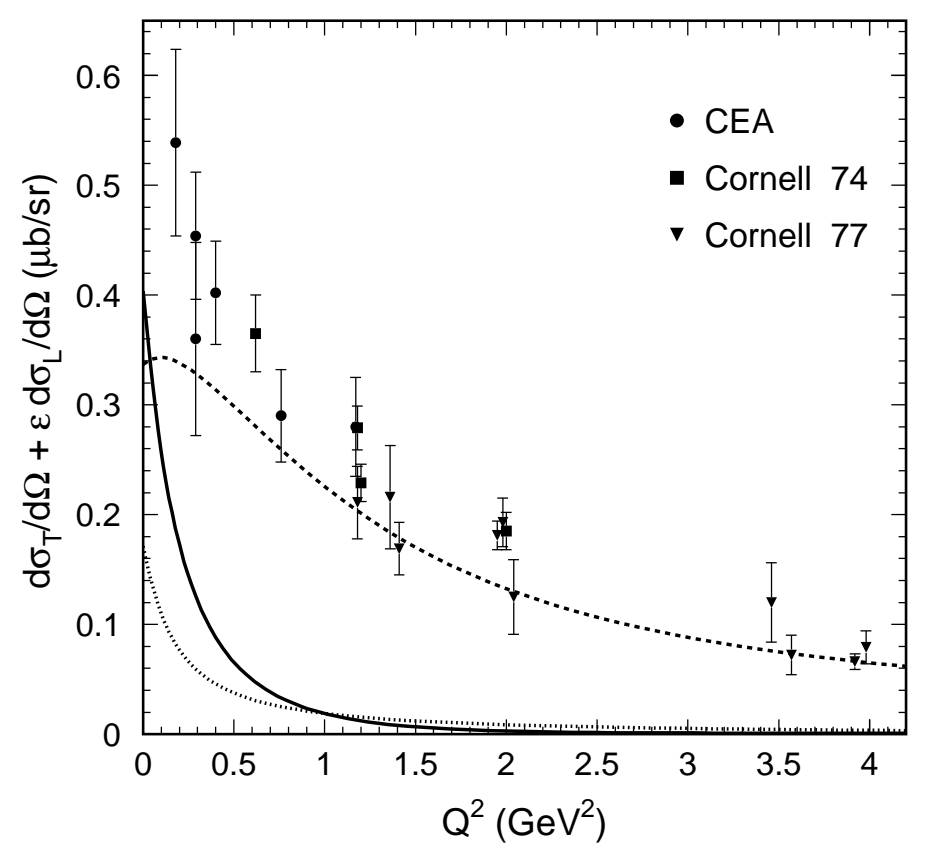

Figure 4.3 Model calculations for the $Q^{2}$-dependence of the $\phi_{K}$-averaged $p\left(e, e^{\prime} K^{+}\right) \Lambda$ differential cross section. The solid, dashed and dotted curves are from the models $\mathrm{A}, \mathrm{B}$ and $C$, respectively. The kinematics are defined by $W=2.15 \mathrm{GeV}, \theta_{\kappa}=8^{\circ}$ and $\epsilon=0.85$. The data are from Refs. [71-73].

again that the models $\mathrm{A}$ and $\mathrm{C}$, which produced fair results in the real photon case, badly fail in describing the electron scattering results.

We feel that the confrontation with the electroproduction data represents a severe test for the different models. There are indications that the procedure of cutting the Born terms, either through the use of soft hadronic form factors (model A) or by strongly reducing the coupling constants (model C), leads to unrealistic predictions as soon as one moves out of the real photon point. Only model B, which adopts $g_{K^{+} \wedge p}$ and $g_{K^{+} \Sigma^{o} p}$ values in conformity with (broken) SU(3) symmetry and cutoff masses corresponding with relatively hard hadronic form factors, succeeds in getting close to the experimentally determined longitudinal strength.

In order to make sure that the models $A$ and $C$ are indeed unable to reproduce the electroproduction $\mathrm{K}^{+} \wedge$ data, we have refitted the parameters for the three mod- 


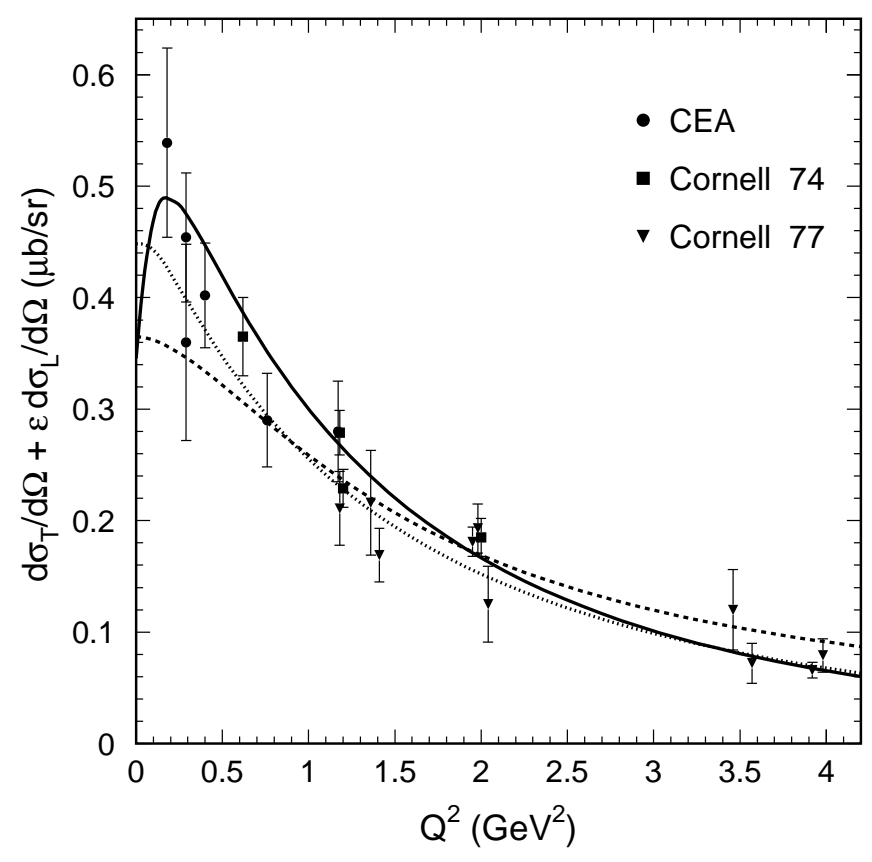

Figure 4.4 $\mathrm{Q}^{2}$-dependence of the $\phi_{\mathrm{K}}$-averaged $p\left(e, e^{\prime} \mathrm{K}^{+}\right) \wedge$ differential cross section. The solid, dashed and dotted curve are from the refitted model $\mathrm{A}, \mathrm{B}$ and $\mathrm{C}$, respectively. The data and kinematics are as in Fig. 4.3.

els against the data of both the real and virtual photoproduction processes. For the virtual photon data, the results from the JLab [70], Cornell [71,72] and CEA [73] experiments are included. For the $\mathrm{K}^{+} \wedge$ case, this amounts to a total of 126 real photon and 34 virtual photon data points. The results of those model calculations with a refitted set of coupling constants, are plotted in Figs. 4.4 and 4.5. For the sum of the longitudinal and transverse part as defined in Eq. (4.25), good results are obtained and the new model calculations are able to reproduce the data. Exploring the separated response functions, though, similar global features, as previously observed, emerge. The predictions for the longitudinal cross sections of model A and C are only marginally enhanced after the refitting procedure and badly undershoot the data. This clearly illustrates the fundamental shortcomings of model A and C. The fact that after the refitting procedure, model $\mathrm{A}$ and $\mathrm{C}$ also fail in describing the longitudinal response points to the fact that $s$-channel resonance contributions cannot 

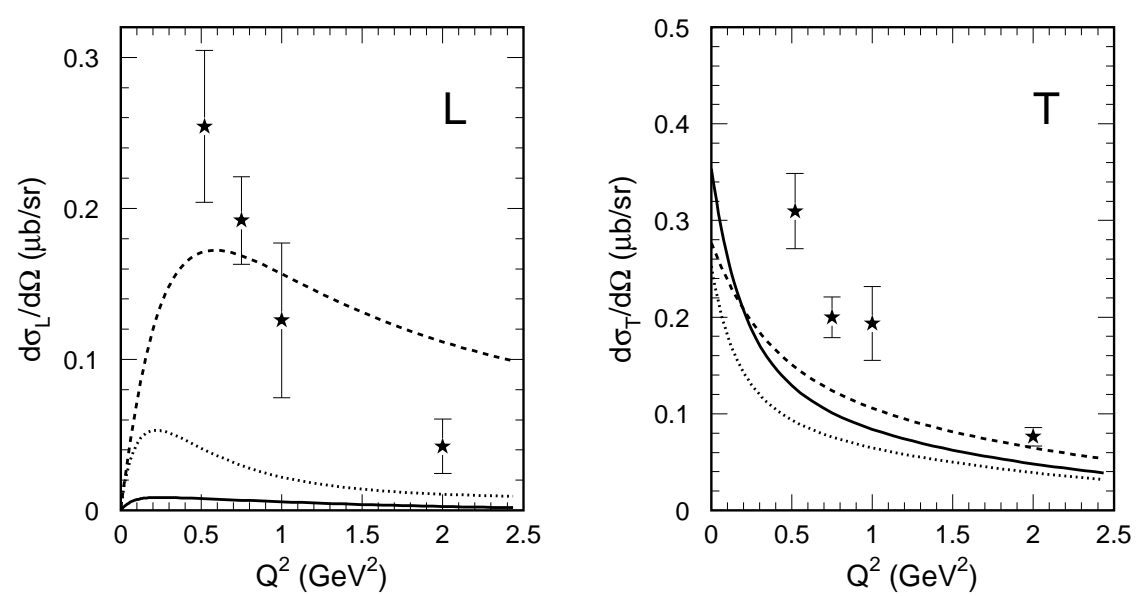

Figure 4.5 $Q^{2}$-dependence of the $d \sigma_{L} / d \Omega$ and $d \sigma_{T} / d \Omega$ cross sections for the $p\left(e, e^{\prime} K^{+}\right) \Lambda$ process. Calculations are obtained with the refitted models and line conventions as in Fig. 4.4. The data and kinematics are as in Fig. 4.2.
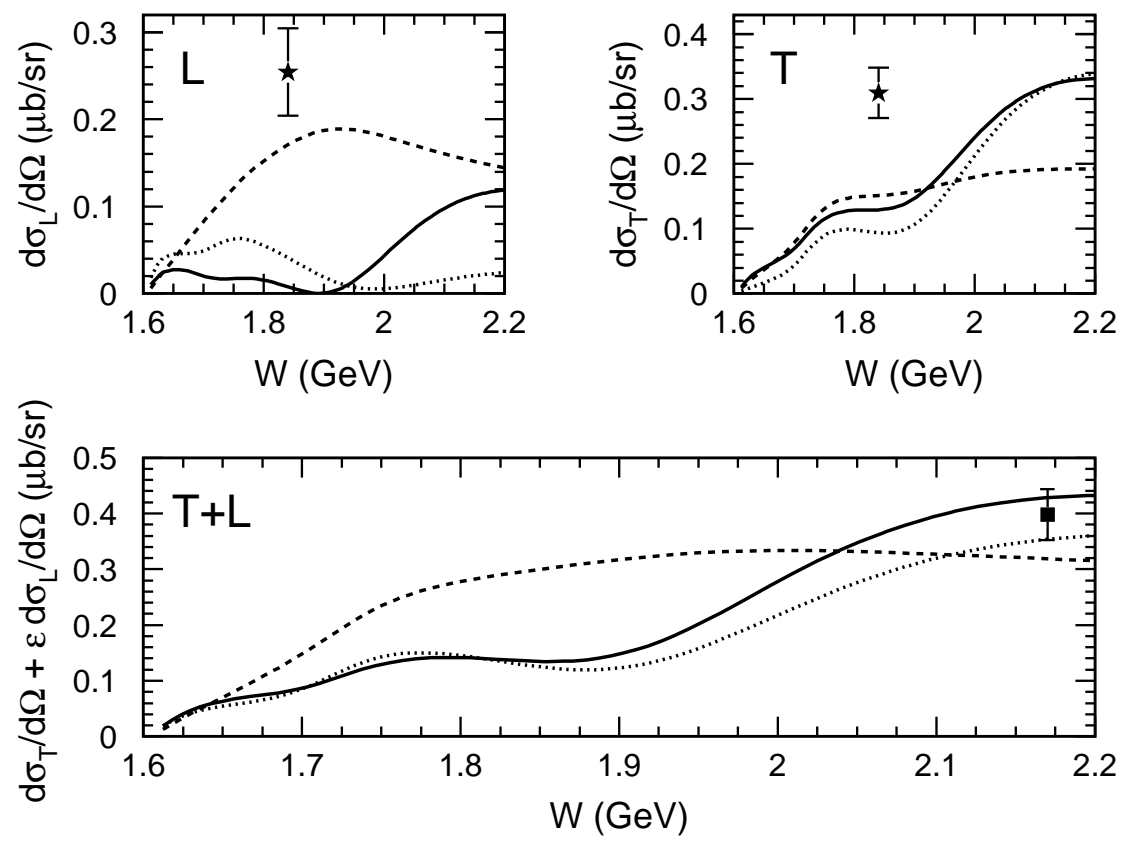

Figure 4.6 $W$-dependence of the $d \sigma_{L} / d \Omega$ and $d \sigma_{T} / d \Omega$ terms and the sum of both for the $p\left(e, e^{\prime} \mathrm{K}^{+}\right) \wedge$ process at $\mathrm{Q}^{2}=0.5 \mathrm{GeV}^{2}$ and $\cos \theta=1$. Calculations are obtained with the refitted models and line conventions as in Fig. 4.4. The data are from Refs. [70,71]. 
held responsible for this underestimation.

It is a remarkable feature that the data of Fig. 4.4 can be reproduced fairly well by all refitted models while the models $\mathrm{A}$ and $\mathrm{C}$ badly fail in reproducing the longitudinal response of Fig. 4.5. First of all, it should be stressed that the Cornell and CEA data of Fig. 4.4 correspond to $W \simeq 2.15 \mathrm{GeV}$ while the JLab data of Fig. 4.5 are taken at $W=1.84 \mathrm{GeV}$. They clearly correspond to a different energy region. To further clarify this issue, we have plotted the $W$-dependence of the response functions in Fig. 4.6. Inspecting those plots, it can be understand why the data points at high $W$ are better accounted for by the three models than the data from the JLab experiment at low $W$. The drop in the longitudinal strength, especially at lower $W$, is indeed responsible for this failure. In general, one can conclude that the refitted models still exhibit a different energy behavior, not only in the $d \sigma_{\mathrm{L}} / \mathrm{d} \Omega$ term but also in the $d \sigma_{\mathrm{T}} / \mathrm{d} \Omega$ part at higher $W$.

\subsubsection{K $\Sigma$ Electroproduction}

In the previous section, we have concentrated on the $p\left(e, e^{\prime} K^{+}\right) \wedge$ process and presented results of model calculations mainly tailored for its real photon counterpart. An analogous analysis can now be performed for the $p\left(e, e^{\prime} K^{+}\right) \Sigma^{0}$ process. However, to our knowledge, there is no published data available for the separated longitudinal $\left(\mathrm{d} \sigma_{\mathrm{L}} / \mathrm{d} \Omega\right)$ and transverse $\left(\mathrm{d} \sigma_{\mathrm{T}} / \mathrm{d} \Omega\right)$ response functions. There exists only measurements for their sum. The predictions for the $d \sigma_{L} / d \Omega$ and $d \sigma_{T} / d \Omega$ terms are presented in Figs. 4.7. The parameters are taken as in the real photon case and the $\mathrm{Q}^{2}$-dependence of the electromagnetic vertices is addressed by a form factor as explained in App. A.3. A striking observation is that the predicted longitudinal $p\left(e, e^{\prime} K^{+}\right) \Sigma^{0}$ strength, in the contrary to the $p\left(e, e^{\prime} K^{+}\right) \wedge$ case, is fairly independent of the model adopted to compute the background diagrams. Note also that in the $\mathrm{K}^{+} \Sigma^{0}$ channel, the contributions from the s-channel resonances is more pronounced than in the $\mathrm{K}^{+} \wedge$ counterpart.

In a subsequent step, we have performed a new fit of the coupling constants, now including the available $p\left(e, e^{\prime} \mathrm{K}^{+}\right) \Sigma^{0}$ data from Cornell [71,72] and the CEA facility [73]. The total fit includes now 130 points for the real photon reactions $\gamma p \rightarrow$ $\mathrm{K}^{+} \Sigma^{0}$ and $\mathrm{K}^{0} \Sigma^{+}$and 24 data points for the ep $\rightarrow e^{\prime} \mathrm{K}^{+} \Sigma^{0}$ process. Within all three models, reasonable overall $\chi^{2}$ values are obtained. The predictions of the refitted 

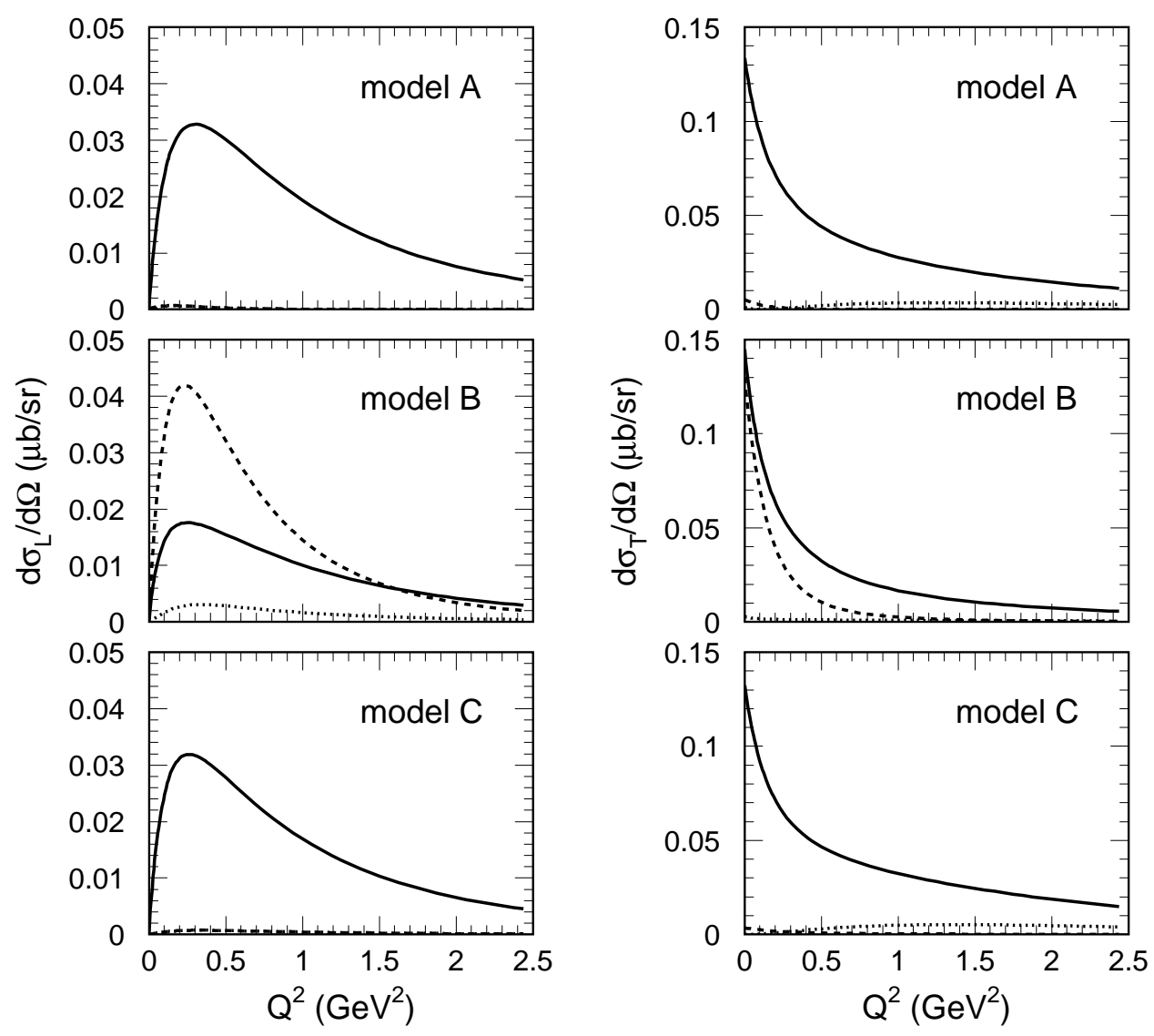

Figure 4.7 $Q^{2}$-dependence of the $d \sigma_{L} / d \Omega$ (left) and $d \sigma_{T} / d \Omega$ (right) response for the $p\left(e, e^{\prime} \mathrm{K}^{+}\right) \Sigma^{0}$ process at $W=1.84 \mathrm{GeV}$ and $\cos \theta=1$. The solid curve is the full calculation. The dashed line is the strength produced by the Born terms with hadronic form factors included. The dotted line represents the contributions from all background diagrams. The panels refer to the three different models (A, B and C) which have been introduced to implement the background diagrams. 


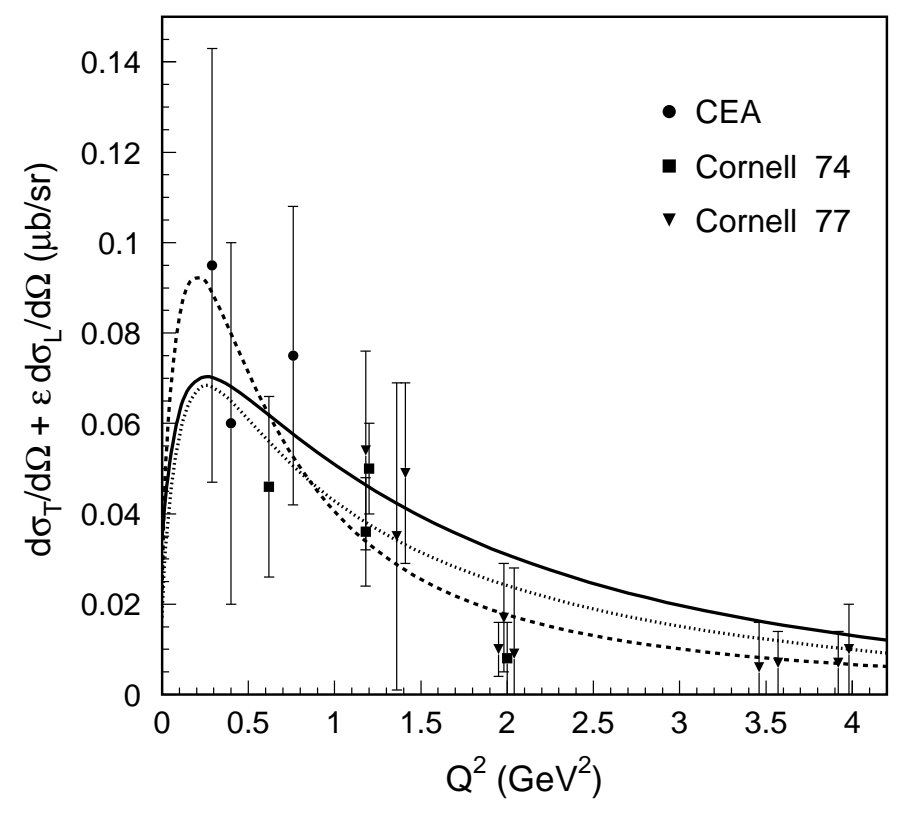

Figure 4.8 Model calculations for the $\mathrm{Q}^{2}$-dependence of the $\phi_{\mathrm{K}}$-averaged $p\left(e, e^{\prime} \mathrm{K}^{+}\right) \Sigma^{0}$ differential cross section. The solid, dashed and dotted curves are from the refitted models $\mathrm{A}$, $\mathrm{B}$ and $\mathrm{C}$, respectively. The kinematics are defined by $\mathrm{W}=2.15 \mathrm{GeV}, \theta_{\mathrm{K}}=8^{\circ}$ and $\epsilon=0.85$. The data are from Refs. [71-73].

model calculations for the $\phi_{\mathrm{K}}$-averaged virtual cross section are collected in Fig. 4.8. It is clear that after the refitting, all three models, primary constructed for the real photon $K \Sigma$ reactions, seem to be in accordance with the available electroproduction $\mathrm{K}^{+} \Sigma^{0}$ data. 



\section{Chapter 5}

\section{Regge Model for KY Production}

Back in 1959, Regge introduced a new concept for dealing with scattering amplitudes [74]. When discussing solutions of the Schrödinger equation for nonrelativistic potential scattering, he suggested to regard the angular momentum $l$ as a complex variable. From quantum mechanical principles, angular momentum is a fundamentally integer quantity. However, Regge showed that an extension into the complex plane could help in determining the dispersion properties of the scattering amplitudes. A few years later, it became clear that this technique of extending angular momentum was extremely useful in high energy particle physics. In fact, it is probably in this branch of science that the idea of a complex angular momentum has been most fruitfully exploited. For a profound discussion of Regge theory and its applications, we refer to the work of Collins [75]. Some excellent reviews can also be found in Refs. [76-78].

A few years ago, Regge theory has experienced a renewed interest in the field of meson production after reports of Regge based models that accurately describe the high energy data $[16,38,39,79]$. Triggered by these observations, in this chapter we will explore the possibility of using Regge theory to account for the global behavior of the $p(\gamma, K) Y$ and $p\left(e, e^{\prime} K\right) Y$ data in the resonance region and beyond. We wish to stress that the investigations reported in this chapter are rather exploratory in nature and that various extensions and modifications of the framework presented here belong to the possibilities for future work.

The organization of this chapter is as follows. In Sec. 5.1 some general concepts of Regge theory are outlined. The results of the model calculations for the photo- 
and electroproduction processes are summarized in Sec. 5.2. Finally, in Sec. 5.3 some limitations and possible extensions of the Regge model are discussed.

\subsection{Regge Theory}

A detailed mathematical outline of the construction of the Regge amplitude for a $a+b \rightarrow c+d$ scattering process is contained in App. C. Here, we will restrict ourselves to sketching the general physical ideas lying behind Regge theory.

As is commonly known, the principle of crossing symmetry predicts that the direct s-channel $(a+b \rightarrow c+d)$ process and its crossed t-channel $(a+\bar{c} \rightarrow \bar{b}+d)$ counterpart can be described by one unique amplitude $M(s, t)$. Obviously, this amplitude has to be evaluated in different regions of the $(s, t)$-Mandelstam plane when describing the two different processes (see Fig. C.1).

Let us now consider the case that the crossed $t$-channel process $a+\bar{c} \rightarrow \bar{b}+d$ is dominated by one single pole. The amplitude $M(s, t)$ can then be appropriately approximated in terms of the partial wave amplitude that includes this pole. This approach will reasonably account for the physical behavior of the (crossed $t$ channel) process. However, when the same amplitude is now used in the physical region of the direct s-channel process $a+b \rightarrow c+d$, as is suggested by crossing symmetry, it turns out that the results have to be handled with the greatest care since it cannot be excluded that one comes across an unphysical divergent behavior. The reason for this failure has to be sought in a convergence problem of the partial wave decomposition for the t-channel amplitude. It can be shown that one goes beyond the convergence domain of the $t$-channel partial wave decomposition when applying it in the s-channel region. Summarizing, it seems that the knowledge of $M(s, t)$ in a certain part of the $(s, t)$-Mandelstam plane does not necessarily imply that this amplitude can be evaluated in any other region of the $(s, t)$-plane.

A possible way to master this difficulty is based on a procedure which sums over all partial waves of the $t$-channel inside the convergence region, before using the amplitude in other parts of the $(s, t)$-Mandelstam plane. To perform this summation, Regge suggested to replace the infinite sum over the discrete eigenvalues of the angular momentum by a contour integral over the complex angular momentum plane. This replacement is referred to as the Sommerfeld-Watson transformation. The contour integral in the complex momentum plane can then be deformed in such 
a manner that a closed expression for the partial wave decomposition is obtained. The resulting expression for the amplitude $M(s, t)$ can now be applied safely in other regions of the Mandelstam plane. To simplify the mathematical descriptions, it is appropriate to focus on the so called Regge limit (high $s$ and small negative $t$ ). Under those conditions, the Regge amplitude for the reaction $a+b \rightarrow c+d$ reads:

$$
M_{\text {Regge }}^{\zeta= \pm}(s, t)=C\left(\frac{s}{s_{0}}\right)^{\alpha(t)} \frac{\beta(t)}{\sin (\pi \alpha(t))} \frac{1+\zeta e^{-i \pi \alpha(t)}}{2} \frac{1}{\Gamma(\alpha(t)+1)} .
$$

For the detailed derivations which lead to this result, we refer the reader to App. C. By construction, the Regge form for the scattering amplitude is unitary and obeys the appropriate analyticity properties. Since the Sommerfeld-Watson transformation of Eq. (C.15) includes all partial waves in the t-channel, the amplitude of Eq. (5.1) is not restricted to the exchange of some isolated poles. To the contrary, a whole family of particles with the same internal quantum numbers as charge, baryon number and strangeness but with different spin, participate in this amplitude. Formally, this is reflected in the exchange of the $\alpha(t)$ "trajectory", which accounts for an entire class of particles. On every occasion that $\alpha(t)$ goes through a (half) integer value, this corresponds with the exchange of an individual particle with a mass $\sqrt{t}$ and spin $\alpha(t)$. In the $s$-channel, however, one has $t \leq 0$ and the individual $t$-channel poles can never be reached. Accordingly, it is the exchange of the whole trajectory that determines the physics of the s-channel reaction now.

As mentioned, a Regge trajectory $\alpha(t)$ connects the spin of a physical particle with its mass squared. When the spins of a set of resonant states are plotted against their mass squared in a so-called Chew-Frautschi plot (see Fig. 5.1), it is phenomenologically observed by visual inspection that all Regge trajectories can be reasonably parameterized by means of a linear function:

$$
\alpha(t)=\alpha_{0}+\alpha^{\prime}\left(t-m_{\alpha_{0}}^{2}\right) .
$$

Here, $\alpha_{0}$ is the spin and $m_{\alpha_{0}}$ the mass of the first materialization of the trajectory. Further, $\alpha^{\prime}$ is the slope of the trajectory which turns out to be close to an universal constant and can be related to a string tension. For all observed baryon and meson trajectories, one has $\alpha^{\prime} \simeq 0.8 \mathrm{GeV}^{2}$. It is believed that this linear behavior and the universal slope of the trajectories reflect the behavior of the underlying partonic degrees-of-freedom of the hadronic spectrum. The trajectories are often named after their first materialization. Examples are the kaon trajectory or the $\mathrm{K}^{*}$ trajectory. 

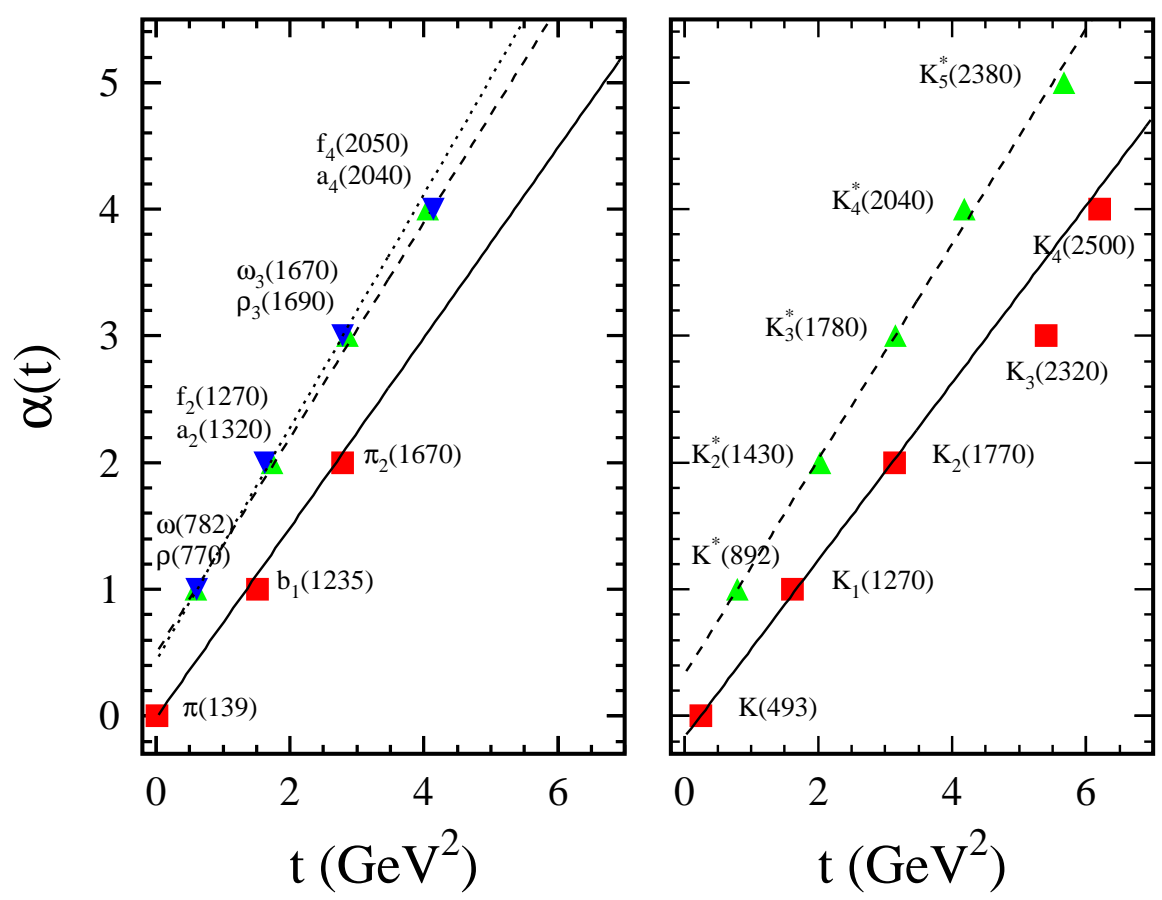

Figure 5.1 The Chew-Frautschi plot for the non-strange and strange mesons. The curves represent the $\pi, \rho$ and $\omega$ trajectories for the $S=0$ case, and the $K$ and the $K^{*}$ trajectories for the $S=1$ sector. 
In the following discussions, we will sometimes refer to this first materialization as the $\alpha_{0}$ particle. This should not be confused with the spin value $\alpha_{0}$ in Eq. (5.2). In the forthcoming, the context will make it clear whether we refer to a physical particle or its spin.

The Regge scattering amplitude as derived in the App. C and given in Eq. (5.1) contains some undetermined quantities like the constant $C$ and the residue function $\beta(t)$. To determine those quantities, one can rely on the fact that in the vicinity of a certain $t$-channel pole $\alpha_{n}$, the Regge amplitude $M_{\text {Regge }}(s, t)$ has to coincide with the Feynman exchange amplitude for this pole:

$$
M_{\text {Feynman }}(s, t) \stackrel{t \rightarrow m_{\alpha_{n}}^{2}}{\longrightarrow} \frac{\beta_{\alpha_{n}}(t)}{t-m_{\alpha_{n}}^{2}} .
$$

Here, $\beta_{\alpha_{n}}(t)$ is defined as the residue of the Feynman amplitude at the pole. In the Regge limit ( $s \gg$ and $|t| \ll$ ), the nearest $t$-channel pole is the one corresponding to the first materialization or $\alpha_{0}$ particle. As such, it is appropriate to compare both descriptions in the vicinity of this pole as a way to identify the $\beta(t)$ function and the constant C. According to Eq. (5.2) and under the assumption that the first materialization is a spin-less particle $\left(\alpha_{0}=0\right)$, there holds that $\alpha(t) \rightarrow 0$ and $\sin (\pi \alpha(t)) \rightarrow \pi \alpha^{\prime}\left(t-m_{\alpha_{0}}^{2}\right)$ in the vicinity of the pole $\alpha_{0}$. By comparing Eq. (5.1) and (5.3), evaluated with those limits, it is easily found that:

$$
\mathrm{C}=\pi \alpha^{\prime}
$$

if the $\beta(t)$ function of the Regge amplitude is taken as the $\beta_{\alpha_{0}}(t)$ residue of the Feynman amplitude. Eventually, with the identification of these two quantities, one arrives at the following form for the Regge amplitude:

$$
M_{\text {Regge }}^{\zeta= \pm}(s, t)=\left(\frac{s}{s_{0}}\right)^{\alpha(t)} \frac{\pi \alpha^{\prime} \beta_{\alpha_{0}}(t)}{\sin (\pi \alpha(t))} \frac{1+\zeta e^{-i \pi \alpha(t)}}{2} \frac{1}{\Gamma(\alpha(t)+1)} .
$$

By construction, this amplitude coincides with the Feynman amplitude at the pole $\alpha_{0}$. When moving away from the pole, deviations between the above expression (5.5) for the scattering amplitude and the Feynman amplitude cannot be ruled out. One advantage of the Regge amplitude is that all the unitary and analyticity conditions are met. Comparing again Eq. (5.3) and (5.5), we can now easily identify everything but the residue function $\beta_{\alpha_{0}}(t)$ as a propagator:

$$
\mathcal{P}_{\text {Regge }}^{\zeta= \pm}(s, t)=\left(\frac{s}{s_{0}}\right)^{\alpha(t)} \frac{\pi \alpha^{\prime}}{\sin (\pi \alpha(t))} \frac{1+\zeta e^{-i \pi \alpha(t)}}{2} \frac{1}{\Gamma(\alpha(t)+1)} .
$$


The Regge propagator $\mathcal{P}_{\text {Regge }}$ replaces the standard $\left(t-m_{\alpha_{0}}^{2}\right)^{-1}$ propagator in the $\alpha_{0}$ exchange Feynman amplitude. As such, one can propose a model in which the operatorial structure of the vertices as determined in an effective Lagrangian approach are maintained and only the propagator is modified. As a consequence of this so called reggeization procedure, the Feynman amplitude corresponding with $\alpha_{0}$ exchange in the $t$-channel effectively incorporates the exchange of a whole class of particles as represented by the trajectory $\alpha(t)$.

In the previous derivation, the constant $C$ and the residue $\beta(t)$ were determined for a scalar $\alpha_{0}$ particle. When the first materialization of the trajectory does carry spin, $\alpha_{0}$ takes on an integer (for mesons) or half-integer (for baryons) value in Eq. (5.2). This effect of spin can be easily incorporated in the Regge propagator by the following replacement:

$$
\alpha(t) \longrightarrow \alpha(t)-\alpha_{0}
$$

This modification ensures that the Regge amplitude matches the corresponding Feynman expression at the appropriate pole of the first materialization where $\alpha(t)=$ $\alpha_{0}$.

\subsection{KY Photo and Electroproduction}

In the previous section, we have identified a Regge propagator which incorporates the exchange of a whole class of particles with the same internal quantum numbers as charge, baryon number and strangeness but with different spin. It can be motivated that the Regge propagator replaces the Feynman propagator in the exchange amplitude of the first materialization. In Ref. [16], it was shown that this reggeization procedure gives rise to fair theoretical predictions for the kaon photoproduction observables. Indeed, the high energy $\mathrm{p}\left(\gamma, \mathrm{K}^{+}\right) \Lambda$ and $\mathrm{p}\left(\gamma, \mathrm{K}^{+}\right) \Sigma^{0}$ photoproduction data at forward angles are well described when the kaon and $K^{*}(892) t$-channel exchange Feynman amplitudes are implemented with the aid of a reggeized propagator. In essence, this reggeization procedure amounts to the following substitution 
when dealing with $\mathrm{p}(\gamma, \mathrm{K}) \mathrm{Y}$ processes:

$$
\begin{aligned}
& \frac{1}{\mathrm{t}-\mathrm{M}_{\mathrm{K}}^{2}} \Rightarrow \\
& \sum_{\zeta_{\mathrm{K}}= \pm} \eta_{\zeta_{\mathrm{K}}}\left(\frac{\mathrm{s}}{\mathrm{s}_{0}}\right)^{\alpha_{\mathrm{K}}(\mathrm{t})} \frac{\pi \alpha_{\mathrm{K}}^{\prime}}{\sin \left(\pi \alpha_{\mathrm{K}}(\mathrm{t})\right)} \frac{1+\zeta_{\mathrm{K}} e^{-i \pi \alpha_{\mathrm{K}}(\mathrm{t})}}{2} \frac{1}{\Gamma\left(1+\alpha_{\mathrm{K}}(\mathrm{t})\right)} \\
& \frac{1}{\mathrm{t}-\mathrm{M}_{\mathrm{K}^{*}}^{2}} \Rightarrow \\
& \sum_{\zeta_{\mathrm{K}^{*}= \pm}} \eta_{\zeta_{\mathrm{K}^{*}}}\left(\frac{\mathrm{s}}{\mathrm{s}_{\mathrm{O}}}\right)^{\alpha_{\mathrm{K}^{*}}(\mathrm{t})-1} \frac{\pi \alpha_{\mathrm{K}^{*}}^{\prime}}{\sin \left(\pi\left(\alpha_{\mathrm{K}^{*}}(\mathrm{t})-1\right)\right)} \frac{1+\zeta_{\mathrm{K}^{*}} e^{-i \pi\left(\alpha_{\mathrm{K}^{*}}(\mathrm{t})-1\right)}}{2} \frac{1}{\Gamma\left(\alpha_{\mathrm{K}^{*}}(\mathrm{t})\right)}
\end{aligned}
$$

The linear trajectories $\alpha_{\mathrm{K}}(\mathrm{t})$ and $\alpha_{\mathrm{K}^{*}}(\mathrm{t})$ are shown in Fig. 5.1 and can be parameterized as:

$$
\begin{aligned}
\alpha_{\mathrm{k}}(\mathrm{t}) & =0.7\left(\mathrm{t}-\mathrm{m}_{\mathrm{K}}^{2}\right) \\
\alpha_{\mathrm{K}^{*}}(\mathrm{t}) & =1+0.85\left(\mathrm{t}-\mathrm{m}_{\mathrm{K}^{*}}^{2}\right)
\end{aligned}
$$

As explained in App. $C$, in determining the Regge propagator one has to distinguish between the two signature parts of the trajectories $(\zeta= \pm)$ in order to obey the convergence criteria. In brief, $\zeta=+$ corresponds with the even and $\zeta=-$ with the odd partial waves. Therefore, the propagator contains a summation over the signature factor $\zeta$. Unfortunately, the theory does not determine the relative sign between the odd and even parts of the trajectory. For that reason, an additional sign, denoted by $\eta_{\zeta}$, needs to be introduced. Obviously, a microscopic description of the trajectories should be able to determine this sign $\eta_{\zeta}$, but at present, there is no firmly established theory which allows to do so. This freedom results in two different solutions for the overall phase:

$$
\frac{1+e^{-i \pi \alpha(t)}}{2} \pm \frac{1-e^{-i \pi \alpha(t)}}{2}= \begin{cases}1 & : \text { constant phase } \\ e^{-i \pi \alpha(t)} & \text { : rotating phase }\end{cases}
$$

As can be inspected, the two different choices for the relative sign $\eta_{\zeta}$ result in a so called constant phase option, identical to 1 and in a rotating phase option, giving rise to the complex factor $e^{-i \pi \alpha(t)}$. The option with the constant phase leaves no imaginary part in the amplitude which would make the target and recoil polarization asymmetries to vanish. Therefore, we do not retain this option. The two 
extreme situations for the overall phase are the result of the fact that the odd $\left(\alpha(t)^{-}\right.$ $=1,3,5 \ldots)$ and even $\left(\alpha(t)^{+}=0,2,4 \ldots\right)$ signature parts of the kaon and $\mathrm{K}^{*}$ trajectory are fully degenerated (see Fig. 5.1). Consequently, both trajectories can be described by one and the same parameterization for $\alpha(t)$ and the amplitudes $M^{\zeta= \pm}(s, t)$ differ only by a phase factor. On the other hand, in case that the odd and even parts are not fully degenerated (see for example the nucleon and $N^{*}(1520)$ trajectory in Fig. 5.8), two different parameterizations $\left(\alpha^{+}(t)\right.$ and $\left.\alpha^{-}(t)\right)$ have to be introduced. In such a case, the amplitudes differ by more than a phase factor and the extreme situation of a purely constant or rotating phase does not occur.

Before discussing the results of the Regge model when describing $p(\gamma, K) Y$ reactions, we comment on the important issue of gauge invariance. Whether or not a reggeized propagator is used, it is well known that the $t$-channel kaon exchange diagram is not gauge invariant. In order to restore this fundamental symmetry at the level of the Feynman diagrams, it is straightforward to prove that an additional (electric) nucleon pole term is also required in the amplitude (see for example the discussion in Sec. 2.2). For that reason, we explicitly include the nucleon pole term in the total amplitude. However, when the kaon propagator is eventually reggeized, it appears natural to apply the same reggeization procedure to the nucleon pole to preserve the correct gauge invariant structure. The reggeization procedure of the kaon amplitude can be formally achieved by multiplying the amplitude determined by standard Feynman diagram techniques, with a factor $\mathcal{P}_{\text {Regge }}^{\mathrm{K}} \cdot\left(\mathrm{t}-\mathrm{m}_{\mathrm{K}}^{2}\right)$. The above recipe was suggested by Guidal et al. in Ref. [16] and eventually results in a total amplitude which takes on a form:

$$
M_{\text {total }}=M_{K}+M_{K^{*}}+M_{p} \cdot \mathcal{P}_{\text {Regge }}^{K} \cdot\left(t-m_{k}^{2}\right) .
$$

Remark that the nucleon pole amplitude $M_{p}$ contains only the electric part proportional to a $\gamma^{\mu}$ coupling. The magnetic coupling, proportional to $\sigma^{\mu \nu}$, is not included on the basis of the duality hypothesis (see App. D). This hypothesis states that either all $t$-channel or all s-channel poles have to be included. A combination of both could lead to a double counting of the poles. As the Regge model takes into account all $t$-channel poles, the amount of $s$-channel contributions is limited to a very strict minimum.

As only two exchange amplitudes are taken into account, those of the kaon and $\mathrm{K}^{*}$, the number of free parameters in the proposed Regge model description of the 


\begin{tabular}{||l|lll||}
\hline \hline$K^{+} \wedge:$ & $g_{K^{+} \wedge p} / \sqrt{4 \pi}=-3.242$ & $G_{K^{*}}^{v}=0.265$ & $G_{K^{*}}^{t}=1.159$ \\
\hline$K^{+} \Sigma^{0}:$ & $g_{K^{+} \Sigma^{0} p} / \sqrt{4 \pi}=1.297$ & $G_{K^{*}}^{v}=0.325$ & $G_{K^{*}}^{t}=-0.766$ \\
\hline \hline
\end{tabular}

Table 5.1 The couping constants obtained from comparing Regge model calculations to the high energy data of Ref. [80]. The coupling constants are defined in App. A.

$p\left(\gamma, \mathrm{K}^{+}\right) \mathrm{Y}$ reactions is limited to three. We have fitted the coupling constants to the high energy $\mathrm{p}\left(\gamma, \mathrm{K}^{+}\right) \Lambda$ and $\mathrm{p}\left(\gamma, \mathrm{K}^{+}\right) \Sigma^{0}$ data at forward angles. The values are summarized in Table. 5.1. Those numbers slightly differ from the ones obtained in Ref. [16] (note that the authors used another definition for the coupling constants). This can mainly be attributed to the difference in the parameterization for the $\mathrm{K}^{*}$ trajectory in Eq. (5.9b). The results of our model calculations are summarized in Fig. 5.2. One can conclude that the data, covering a large energy range (5 GeV $\left.\leq \omega_{\mathrm{lab}} \leq 16 \mathrm{GeV}\right)$, are fairly well described. Further, it is observed that the contribution of the $\mathrm{K}^{*}$ trajectory accounts for the major part of the computed strength. Only for the $p\left(\gamma, K^{+}\right) \wedge$ process at small $t$, the kaon trajectory provides most of the strength.

As already outlined in Ref. [38,39], a Regge model description on the basis of Eq. (5.11) also succeeds to satisfactorily account for the electroproduction data. The results of our model calculations are shown in Figs. 5.3 and 5.4. Thereby, the $\mathrm{Q}^{2}$-dependence of the electromagnetic couplings was materialized through the introduction of electromagnetic dipole form factors with a cutoff of $1.2 \mathrm{GeV}$. Both the older data [71-73] for $d \sigma_{T}+\epsilon d \sigma_{L}$, as well as the new JLab data [70] for the separated transverse $\left(\mathrm{d} \sigma_{\mathrm{T}}\right)$ and longitudinal $\left(\mathrm{d} \sigma_{\mathrm{L}}\right)$ response, are reasonably well described.

The results presented so far, indicate that a Regge based model naturally reproduces the correct $s$ and $t$ dependence of the $p(\gamma, K) Y$ data in the high energy domain at forward angles. Moreover, the small number of free parameters is a clear asset of this approach. With only three coupling constants, the data for each isospin channel can be described. An important additional observation is that the $\mathrm{g}_{\mathrm{K}^{+}} \wedge_{\mathrm{p}}$ and $g_{K+\Sigma^{0} p}$ coupling constants, governing the strength of the kaon trajectory, are 

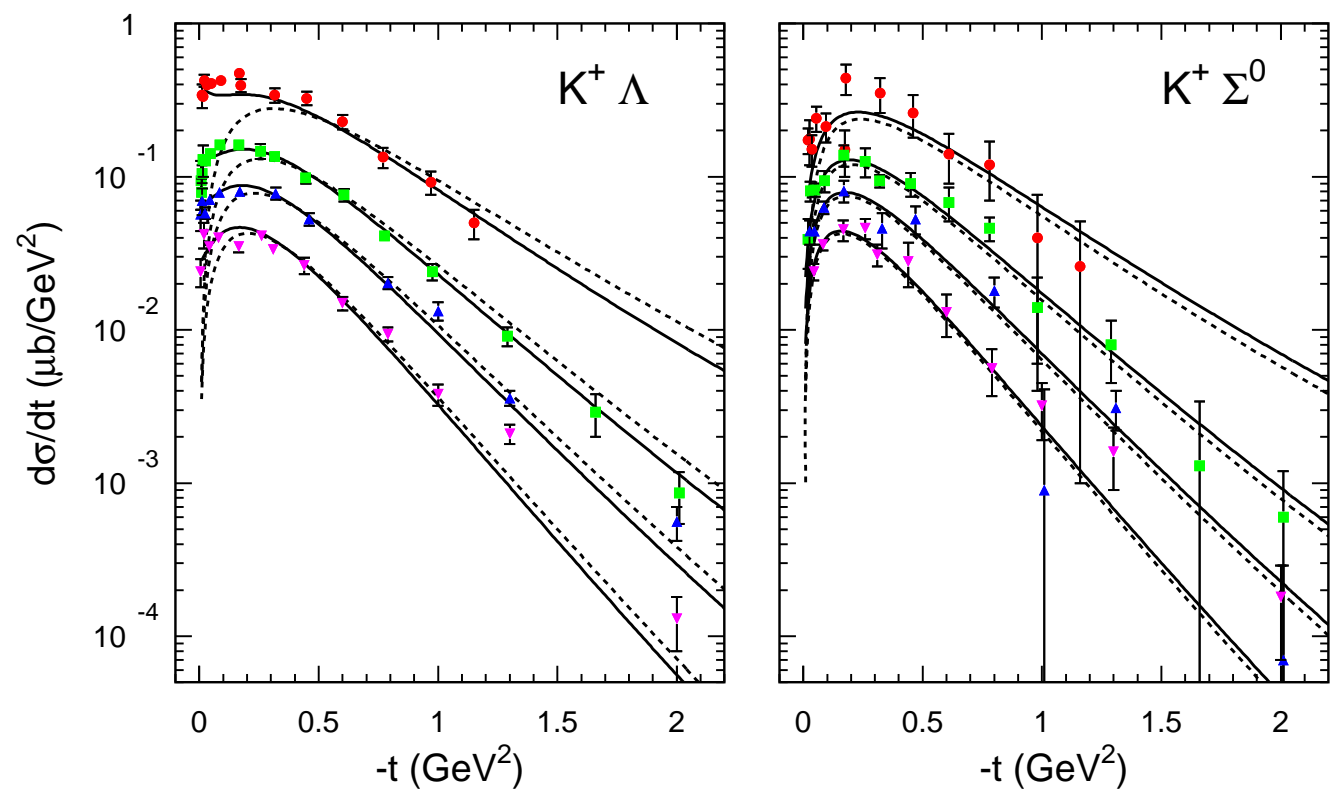

Figure 5.2 The $t$-dependence of the differential $p\left(\gamma, K^{+}\right) \Lambda$ and $p\left(\gamma, K^{+}\right) \Sigma^{0}$ cross sections for various photon lab energies. The solid line includes both the $\mathrm{K}$ and $\mathrm{K}^{*}$ trajectories. The dashed line includes only the $\mathrm{K}^{*}$ part. The data are from Ref. [80] and account for photon lab energies of $5(\mathbf{\bullet}), 8(\mathbf{\square}), 11(\mathbf{\Delta})$ and $16(\boldsymbol{\nabla}) \mathrm{GeV}$. 


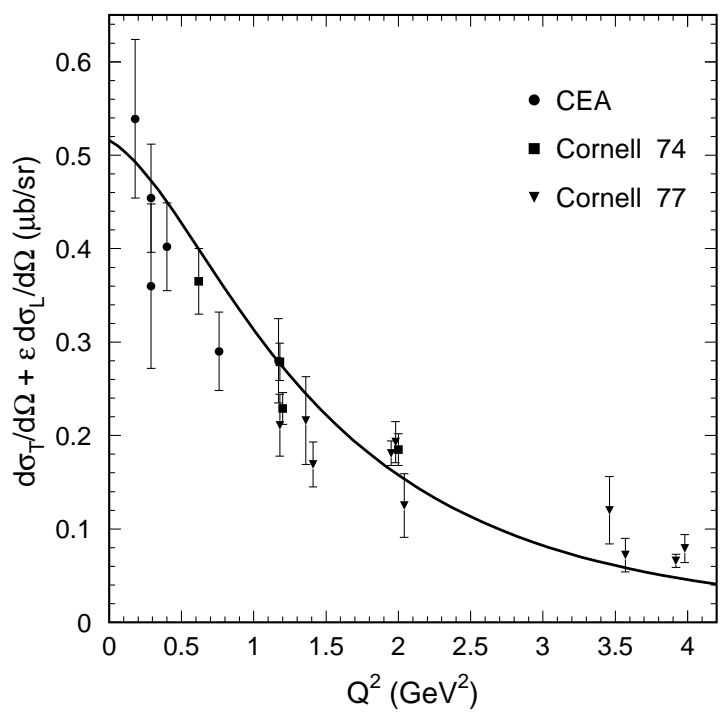

Figure 5.3 A Regge model calculation for the $\mathrm{Q}^{2}$-dependence of the $\phi_{\mathrm{K}}$-averaged differential $p\left(e, e^{\prime} \mathrm{K}^{+}\right) \wedge$ cross section. The kinematics are defined by $W=2.15 \mathrm{GeV}, \theta_{\mathrm{K}}=8^{\circ}$ and $\epsilon=$ 0.85. The data are from Refs. [71-73].
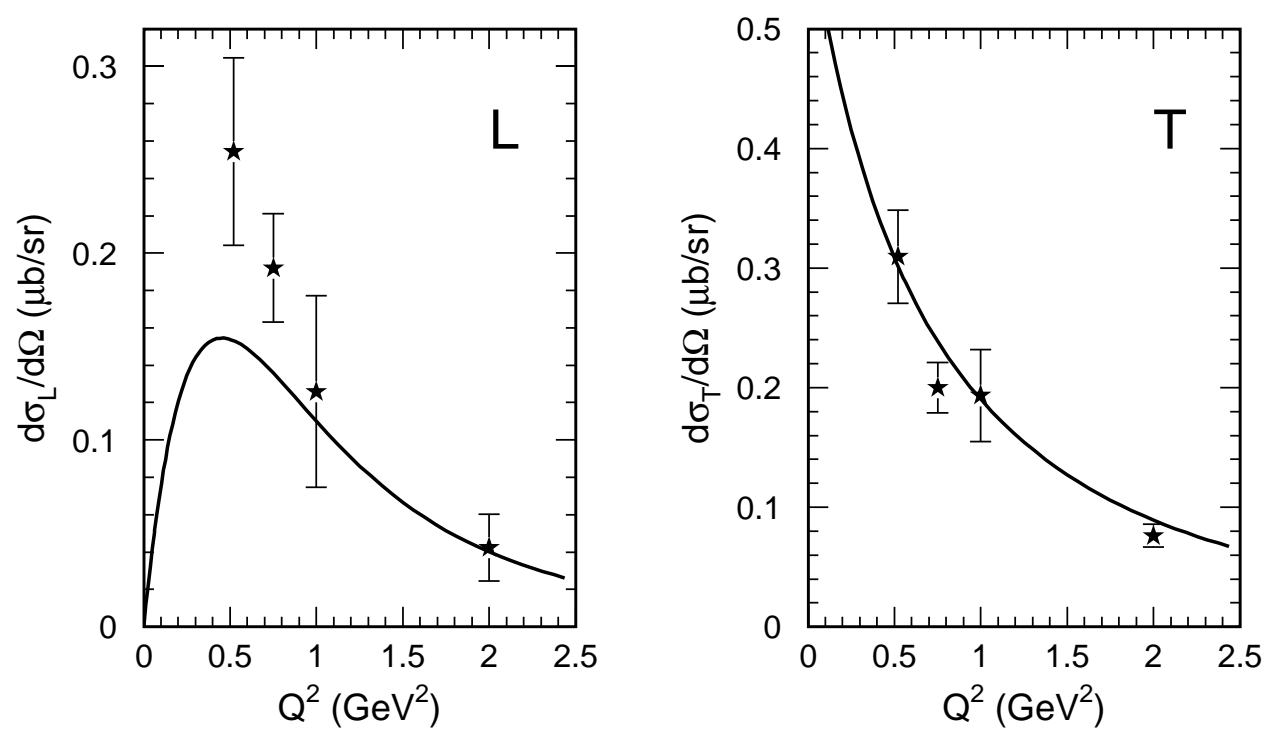

Figure 5.4 A Regge model calculation for the $\mathrm{Q}^{2}$-dependence of the longitudinal (left) and transverse (right) $p\left(e, \mathrm{eK}^{+}\right) \wedge$ response. The data are from Ref. [70] and correspond with $W$ $=1.84 \mathrm{GeV}$ and $\cos \theta_{\mathrm{K}}=1$. 
in accordance with predictions based on a rather modest breaking of SU(3)-flavor symmetry.

It is interesting to explore the possibility of extending the Regge model into the resonance region. There are various motivations for doing this. First, the small number of free parameters is remarkable. In isobar models, one is typically dealing with 15 free parameters or more when attempting to describe the same type of reactions. More important is the fact that Regge theory naturally bridges the gap between the observed hadrons and the underlying parton degrees-of-freedom that constitute the basis of the meson and baryon trajectories. In this respect, the theory offers excellent opportunities to connect the $\mathrm{p}(\gamma, \mathrm{K}) \mathrm{Y}$ observables (in the resonance region) with the more fundamental QCD based degrees-of-freedom.

Strictly speaking, the Regge propagator of Eq. (5.6), as it has been derived in App. C, is only valid in the limit of large values for s. Therefore, one cannot expect miraculous results of the theory in the resonance region. Despite those reservations, we simply took the parameters determined in the high energy regime and computed the $\mathrm{p}\left(\gamma, \mathrm{K}^{+}\right) \Lambda$ and $\mathrm{p}\left(\gamma, \mathrm{K}^{+}\right) \Sigma^{0}$ cross sections in the resonance region. The results of these numerical calculations are compared to the measured total cross sections and presented in Fig. 5.5. Not unexpectedly, the quality of agreement with the data is inferior to what was observed at higher energies. Nevertheless, in the light of the parameter-free nature of the model, the overall agreement is not bad either. For the $\mathrm{p}\left(\gamma, \mathrm{K}^{+}\right) \Sigma^{0}$ process, the energy dependence of the data is reasonably well reproduced. In the $p\left(\gamma, \mathrm{K}^{+}\right) \wedge$ channel, the model calculations overshoot the data by a factor of two. Our Regge based prediction of the $\mathrm{K}^{+} \Sigma^{0}$ cross section in the resonance region differs from the one obtained with the parameterization of Guidal et al. in Ref. [16]. The variations can be traced back to differences in the coupling constants and different parameterizations for the $\mathrm{K}^{*}$ trajectory. From Fig. 5.6, one notices that without any adjustment in the parameters, the Regge model also succeeds in giving a fair account of the recoil polarization data at forward angles. For example, the difference in sign at forward angles between the $K^{+} \Lambda$ and $K^{+} \Sigma^{0}$ channel is naturally explained. In addition, Fig. 5.7 collects some model predictions for the photon beam asymmetry. At present, no published data are available for this observable but preliminary results of GRAAL and SPring- 8 all points towards a positive sign in both the $\mathrm{K}^{+} \Lambda$ and $\mathrm{K}^{+} \Sigma^{0}$ channel $[59,60]$. At backward angles, the 

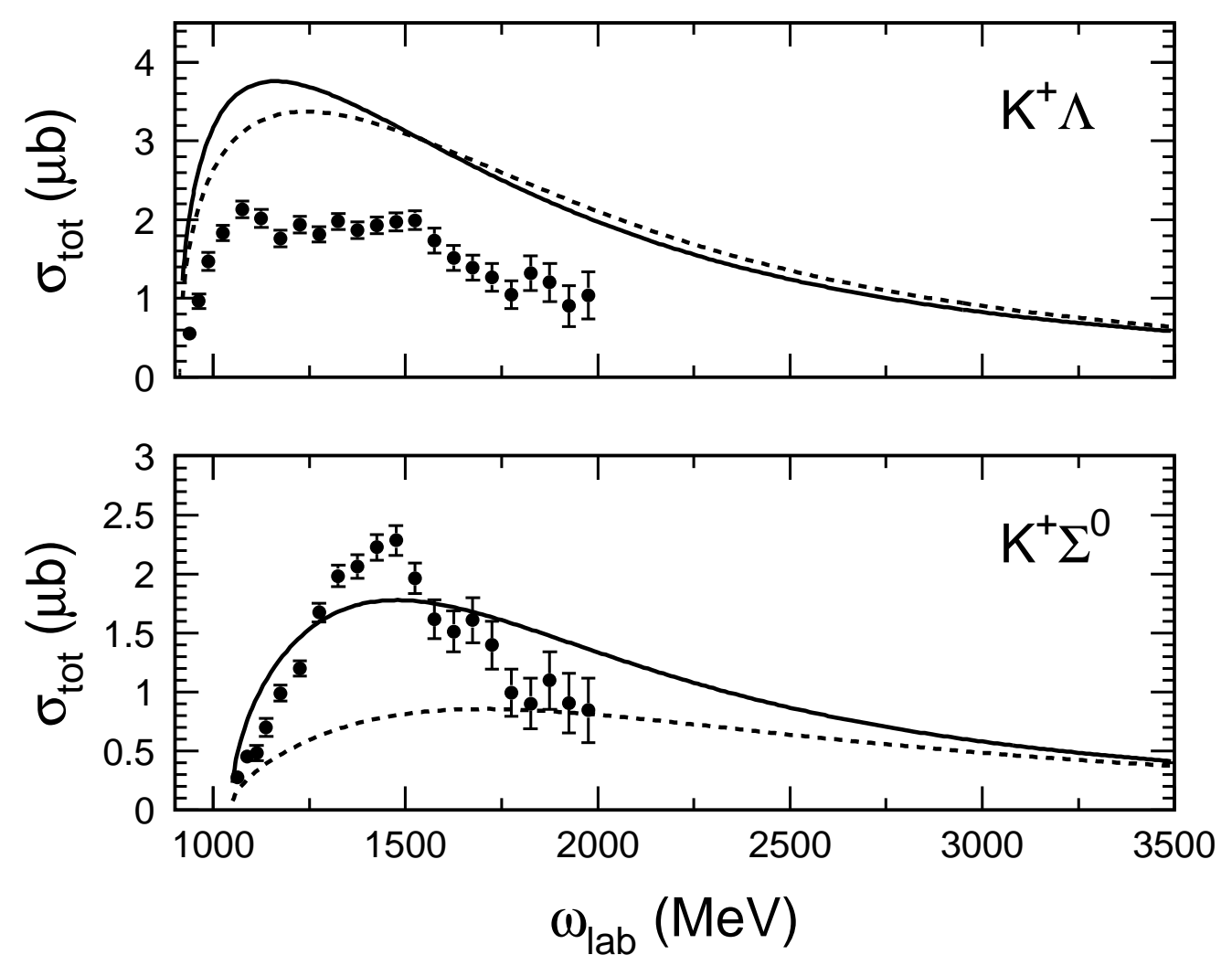

Figure 5.5 Photon energy dependence of the total $p\left(\gamma, \mathrm{K}^{+}\right) \Lambda$ and $p\left(\gamma, \mathrm{K}^{+}\right) \Sigma^{0}$ cross section. The solid line is a Regge model calculation with the parameterizations of this work, the dashed line is obtained with the values of Ref. [16]. The data are from Ref. [23]. 


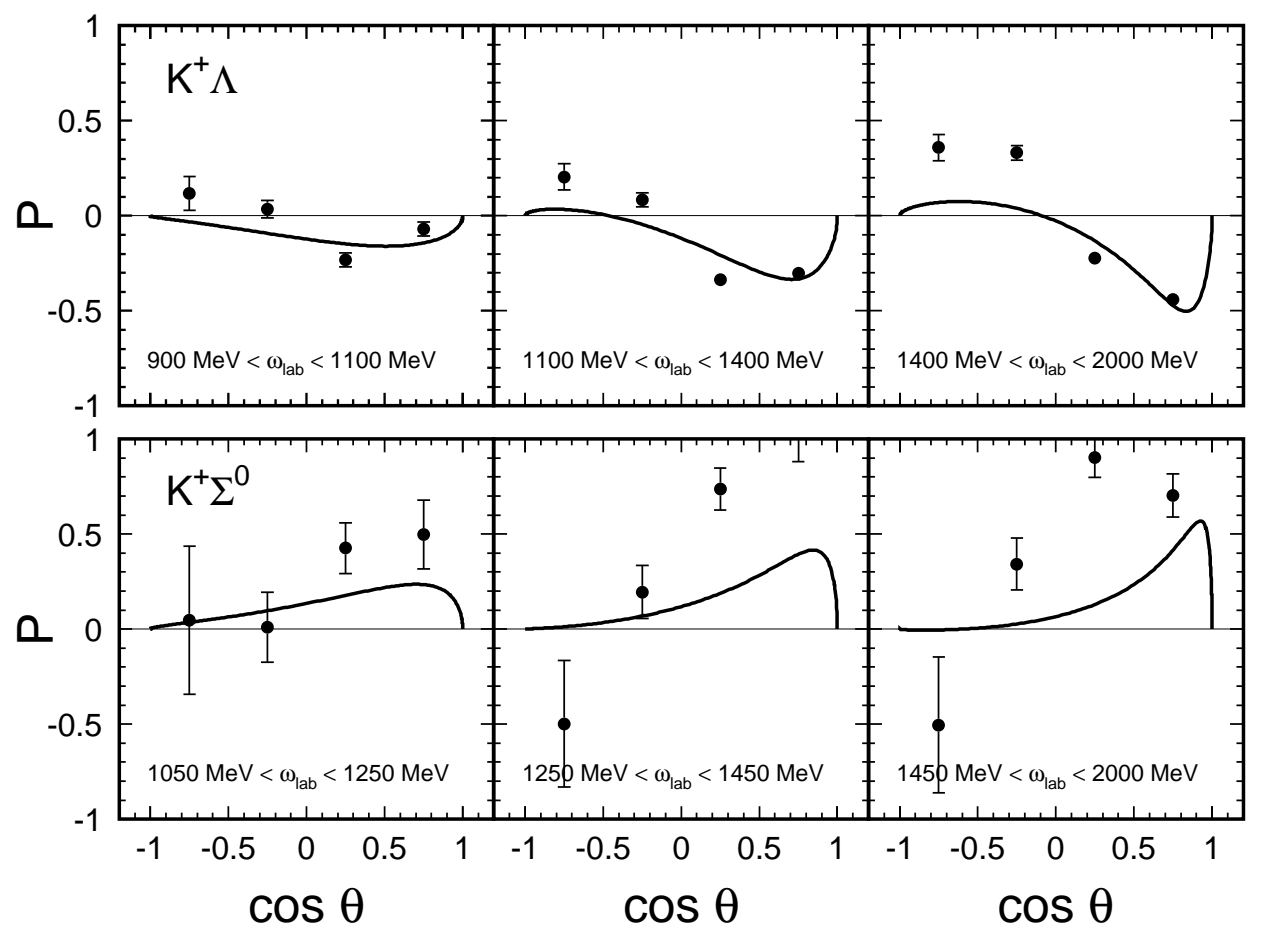

Figure 5.6 A Regge model calculation of the angular dependence of the recoil polarization asymmetry for the $p\left(\gamma, K^{+}\right) \vec{\Lambda}$ (upper panels) and $p\left(\gamma, K^{+}\right) \vec{\Sigma}^{0}$ (lower panels) process. The data are from Ref. [23].

Regge model calculations result in a vanishing asymmetry. This is not surprising given that the Regge amplitude of Eq. (5.5) was derived for small $t$, or, forward angles. Remark that at backward angles, a $u$-channel reggeization procedure could be appropriate.

\subsection{Extensions of the Regge Model}

\subsubsection{Duality Corrections}

In the previous section, exploratory Regge based calculations in the resonance region were reported. Such a description based on t-channel dominance, will intrinsically not be able to account for resonant s-channel structures in the energy dependence of the observables. At best, a Regge description can only be expected 

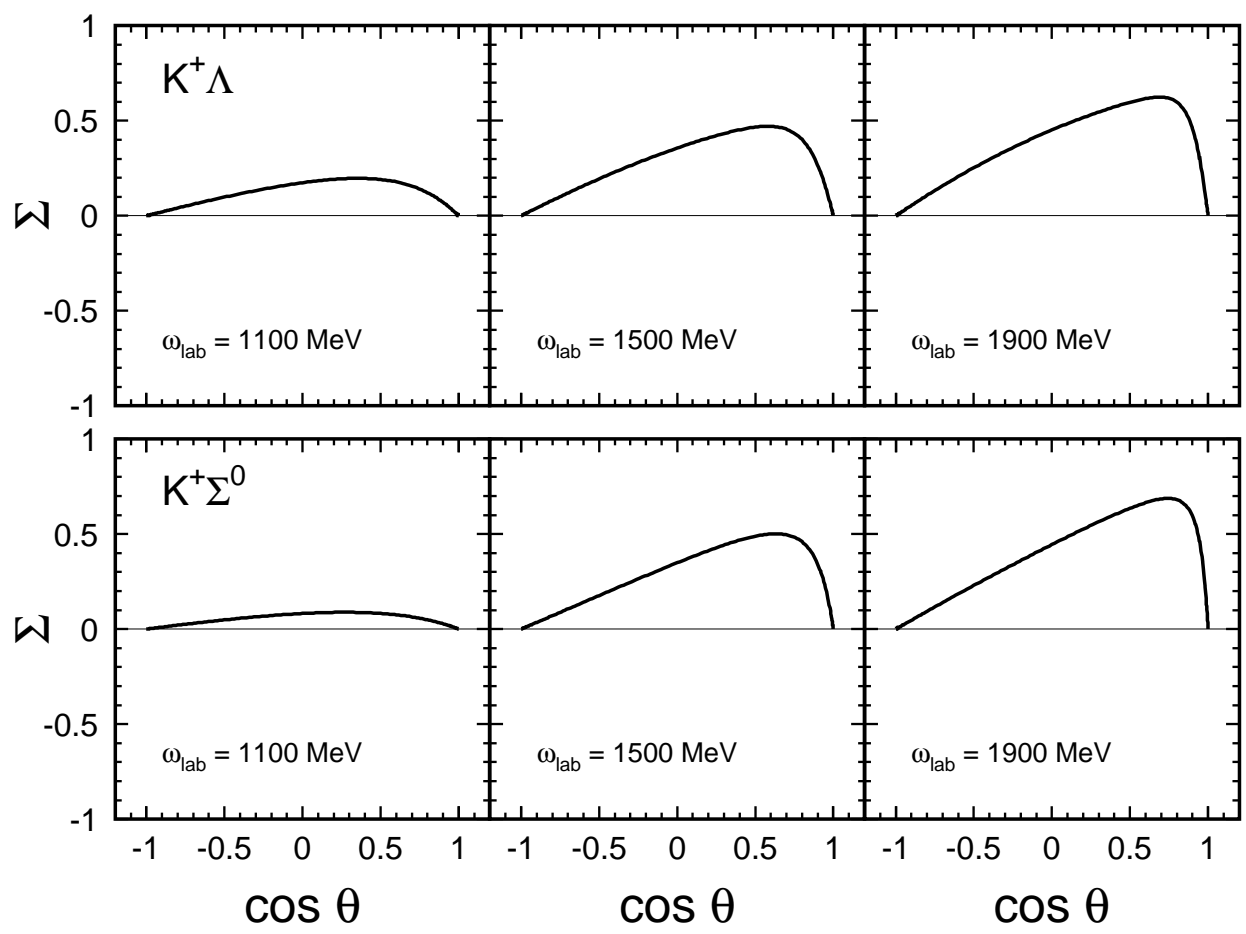

Figure 5.7 Regge model calculation of the angular dependence of the photon beam asymmetry for the $\mathrm{p}\left(\vec{\gamma}, \mathrm{K}^{+}\right) \wedge$ (upper panels) and $\mathrm{p}\left(\vec{\gamma}, \mathrm{K}^{+}\right) \Sigma^{0}$ (lower panels) process.

to provide an overall description of the physics in the resonance region. This is not surprising, given that the theory does not contain any explicit information on specific s-channel resonances. Therefore, in a first step, one can think of using a Regge model to constrain the background diagrams and adding resonance contributions on top of it. However, in doing so, one runs into a situation which clashes with the duality hypothesis [75]. In Regge theory, all t-channel poles are taken into account and when additional s-channel resonances are introduced, the possibility exists that the poles are doubly counted.

A way to master this duality difficulty was suggested by Veneziano [81]. He constructed a phenomenological model in which both $s$ - and $t$-channel resonances were included, at the same time excluding any possible source of double counting. The Veneziano model turns out to produce reaction amplitudes which exhibit ordinary t-channel Regge behavior. In addition, the presence of s-channel poles gives 
rise to an additional term in the phase factor of the scattering amplitude which translates itself in the following substitution for the Regge amplitude (5.5):

$$
1+e^{-i \pi \alpha(t)} \longrightarrow 1+e^{-i \pi \alpha(t)}+\sin (\pi \alpha(t)) \frac{1+e^{i \pi \alpha(s)}}{\sin (\pi \alpha(s))} .
$$

In what follows, we will refer to the last term as the duality correction. The essential features and assumptions of the Veneziano model are summarized in App. D. Despite the fact that it is based on rather severe assumptions, the Veneziano model possesses some interesting features. In the literature, the model has been applied to a variety of reactions [82-84]. In general, however, the "bare" Veneziano amplitude appears too restrictive to provide a realistic description of the production processes in the resonance region [75]. Nevertheless, the additional term in Eq. (5.12) can be considered as a characteristic feature of all dual models [84]. Therefore, we will use this prescription in the Regge formalism in order to introduce direct s-channel poles in a way that respects the duality between the (t-channel) Regge and the ( $s$ channel) resonance amplitude.

When s-channel poles are introduced, it is appropriate to take into account the finite lifetime of those resonances. Therefore, we introduce an imaginary part for the $\alpha(s)$ trajectory:

$$
\begin{aligned}
\alpha(s) & =\alpha_{n}+\alpha^{\prime}\left(s-s_{\alpha_{n}}\right)+i \operatorname{Im}\{\alpha(s)\} \\
& =\alpha_{n}+\alpha^{\prime}\left(s-s_{\alpha_{n}}+i \sqrt{s_{\alpha_{n}}} \frac{\operatorname{Im}\{\alpha(s)\}}{\sqrt{s_{\alpha_{n}}} \alpha^{\prime}}\right)
\end{aligned}
$$

Here, $\alpha_{n}$ is the spin of a pole with mass $\sqrt{s_{\alpha_{n}}}$. The above equation can be made to fit the well known Breit-Wigner form for the propagator if the following association is made:

$$
\operatorname{Im}\{\alpha(s)\}=\alpha^{\prime} \sqrt{s_{\alpha_{n}}} \Gamma_{\alpha_{n}}(s) .
$$

The real and imaginary parts of the $\alpha(\mathrm{s})$ trajectories for the $\mathrm{N}^{*}$ and $\Delta^{*}$ s-channel resonances are displayed in Fig. 5.8. For the imaginary part of the $\alpha(s)$ trajectories, we have used the following parameterizations:

$$
\begin{aligned}
& \operatorname{Im}\left\{\alpha_{N^{*}}(s)\right\}=-0.85+0.37 \mathrm{~s}, \\
& \operatorname{Im}\left\{\alpha_{\Delta^{*}}(\mathrm{~s})\right\}=-0.20+0.20 \mathrm{~s} .
\end{aligned}
$$



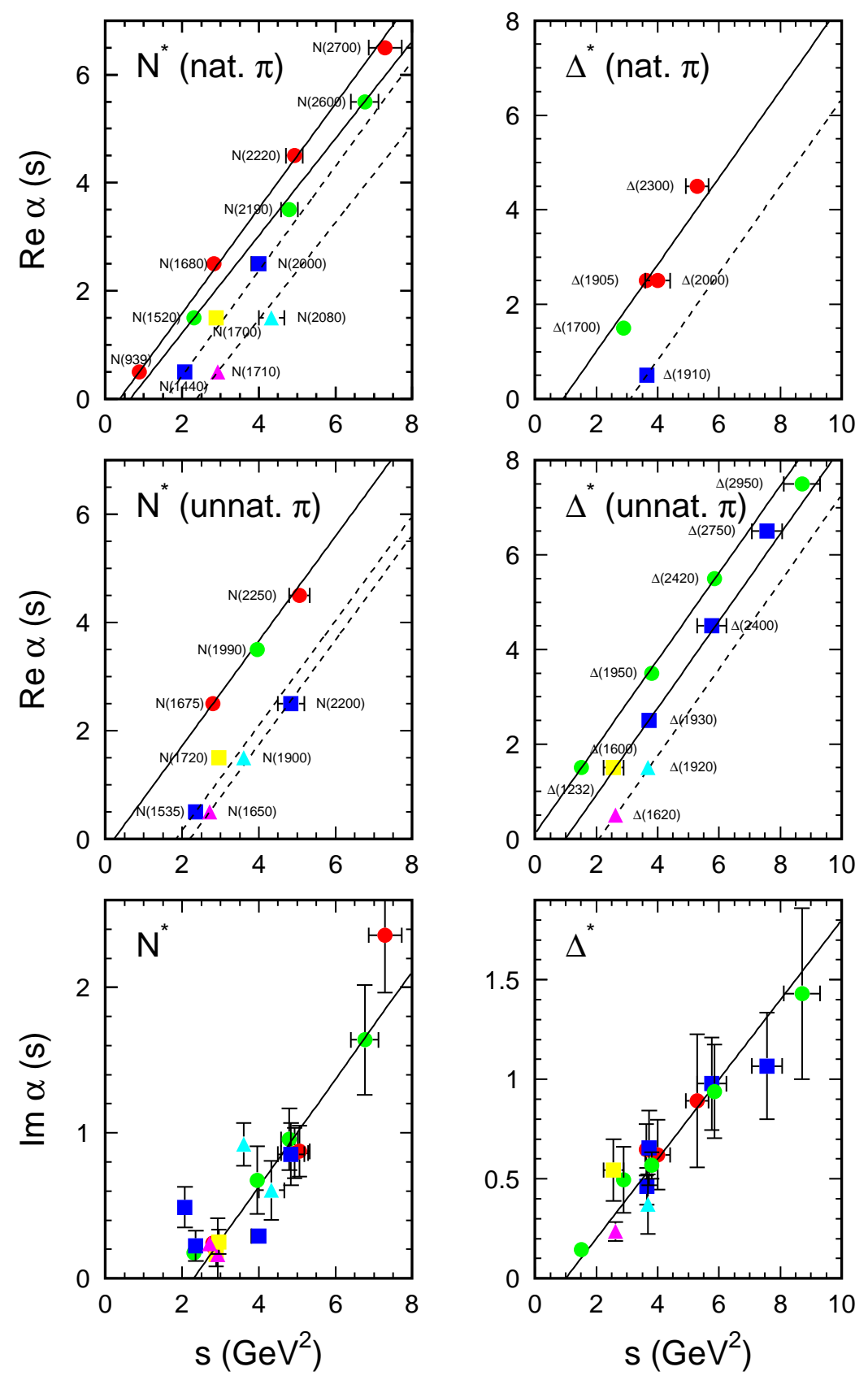

Figure 5.8 The real and imaginary part of the $\alpha(\mathrm{s})$ trajectories of the nucleon and $\Delta^{*}$ resonances. In the real part, distinction is made between the resonances with natural and unnatural parity. The trajectories depicted by a solid line are taken into account in the $p(\gamma, \mathrm{K}) \mathrm{Y}$ calculations based on the Veneziano model. 
Note that the relatively large error bars on the experimental widths, contained in the bottom panels of Fig. 5.8, do not put stringent constraints on these parameterizations. Thereby, it should be stressed that an observable impact on the predictions is noticed for modest variations in the parameters of the expressions (5.15). The slope of the real part is for all trajectories of the order $0.9 \mathrm{GeV}^{-2}$. According to the general expression (D.11) and in order to guarantee that the correct resonance structure at the poles is imposed, the $\alpha$ (s) trajectories that enter Eq. (5.12) are subject to a shift according to the spin of the first materialization:

$$
\alpha(s) \longrightarrow \alpha(s)-\alpha_{0} .
$$

Considering the duality correction of Eq. (5.12) proposed by Veneziano, the Regge propagator of Eq. (5.6), gains an additional term in the phase factor and takes on the form:

$$
\begin{aligned}
\mathcal{P}_{\text {Ven }}(s, t)= & \left(\frac{s}{s_{0}}\right)^{\alpha(t)} \frac{\pi \alpha^{\prime}}{\sin (\pi \alpha(t))} \frac{1}{\Gamma(1+\alpha(t))} \frac{1}{2}\left[1+\zeta_{t} e^{-i \pi \alpha(t)}\right. \\
& \left.-i \zeta_{t} \zeta_{s} \sin (\pi \alpha(t)) \frac{e^{i \pi \alpha_{R}(s)}-\zeta_{s} e^{-\pi \alpha_{I}(s)}}{\cosh \left(\pi \alpha_{I}(s)\right)-\zeta_{s} \cos \left(\pi \alpha_{R}(s)\right)}\right] .
\end{aligned}
$$

Here, the duality correction term is rewritten in order to make the dependence on the real and imaginary part of $\alpha(s)=\alpha_{R}(s)+i \alpha_{I}(s)$ more explicit. We have also introduced the appropriate signature factors $\zeta_{s}$ and $\zeta_{t}$ for the $s$ - and $t$-channel trajectories. The behavior of the real and imaginary part of the duality correction term (for $\zeta_{s}=+$ ) are plotted in Fig. 5.9a. From those surfaces, it becomes obvious that for a vanishing imaginary part $\alpha_{\mathrm{I}}(s)$, the resonant structure of the duality term is revealed. If no width would be associated to the trajectory $\alpha(s)$, the duality term would simply introduce additional s-channel Breit-Wigner resonances, when $\alpha(s)$ moves through the half integer values $1 / 2,5 / 2,9 / 2 \ldots$. Note that the negative signature $\left(\zeta_{s}=-\right)$ part produces poles at $\alpha(s)=3 / 2,7 / 2 \ldots$. The appearance of an imaginary part in $\alpha(s)$ guarantees that the global effect of the duality term remains confined to the resonance region. On the basis of the parameterizations of the $\alpha_{\mathrm{I}}(\mathrm{s})$ in Eq. (5.15), it is found that towards the tail of the resonance region $(s \simeq$ $\left.7 \mathrm{GeV}^{2}\right)$ one has $\alpha_{\mathrm{I}}(\mathrm{s}) \simeq 1.5$. For this value of $\alpha_{\mathrm{I}}(\mathrm{s})$, visual inspection of Fig. 5.9 learns that both the real and imaginary part of the duality term become negligible.

From the above discussion, it emerges that the Veneziano procedure is able to introduce the physically relevant s-channel resonant contributions into a t-channel 

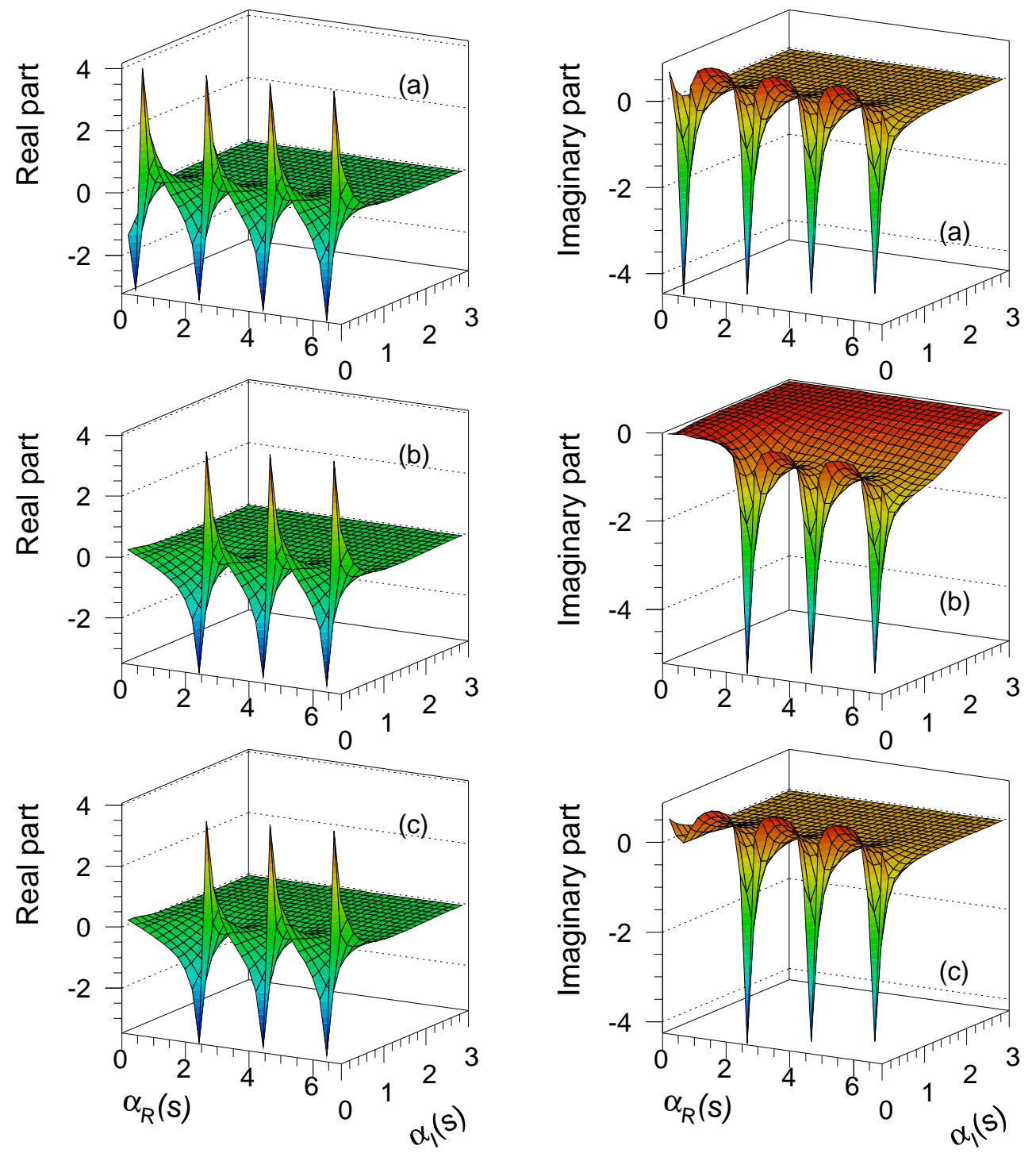

Figure 5.9 The real and imaginary part of the $\left(\zeta_{s}=+\right)$ duality correction term (with exception of the $\sin (\pi \alpha(t))$ factor) to the Regge propagator. Panels (a) contain the unmodified $[1+\exp (i \pi \alpha(s))] / \sin (\pi \alpha(s))$ term. In the panels $(b)$, the nucleon pole at $\alpha_{R}(s)=1 / 2$ is subtracted in order to avoid double counting. Finally, in the panels (c), a correction for the unphysical offset in the imaginary part of panel $(b)$ is made. 
dominated Regge model. Thereby, the major concern of the duality hypothesis, namely not to double count poles, is properly imposed. With respect to this double counting, there remains one difficulty. Indeed, by adding $s$-channel poles at every half integer value, also the nucleon pole at $\alpha_{R}(s)=1 / 2$ gets introduced. As explained in the discussion preceding the Eq. (5.11), the requirement of gauge invariance makes one to add a nucleon pole term (with a reggeized propagator) to the total reaction amplitude. To avoid double counting, one has to subtract this nucleon pole contribution from the duality correction term. This subtraction is illustrated in Fig. 5.9b, where indeed the Breit-Wigner resonance structure is missing at $\alpha_{R}(s)=1 / 2$. Mathematically, this procedure amounts to subtracting in Eq. (5.17) the term:

$$
\begin{gathered}
\zeta_{t} \sin (\pi \alpha(t))\left[\frac{\pi \alpha_{R}^{\prime}\left(s-m_{p}^{2}\right)}{\cosh \left(\pi \alpha_{I}(s)\right)-1+\frac{1}{2}\left[\pi \alpha_{R}^{\prime}\left(s-m_{p}^{2}\right)\right]^{2}}\right. \\
\left.-i \frac{\left.1-\frac{1}{2}\left[\pi \alpha_{R}^{\prime}\left(s-m_{p}^{2}\right)\right]^{2}-e^{-\pi \alpha_{I}(s)}\right]}{\cosh \left(\pi \alpha_{I}(s)\right)-1+\frac{1}{2}\left[\pi \alpha_{R}^{\prime}\left(s-m_{p}^{2}\right)\right]^{2}}\right] .
\end{gathered}
$$

The functional form of this term which subtracts the nucleon pole contribution is obtained when expanding the duality correction term about the nucleon pole $\alpha_{R}(s)$ $=1 / 2$. According to Eq. (5.16), the functions $\cos \left(\pi \alpha_{R}(s)\right)$ and $\exp \left(i \pi \alpha_{R}(s)\right)$ in Eq. (5.17) receive a spin shift $-1 / 2$ in $\alpha_{R}(s)$ before they are evaluated. This makes that the nucleon pole corresponds to:

$$
\alpha_{R}(s)-\frac{1}{2}=\pi \alpha_{R}^{\prime}\left(s-m_{p}^{2}\right)=0,
$$

as one should expect. Consequently, one can rely on $\cos x \simeq 1-\frac{1}{2} x^{2}$ and $e^{i x} \simeq$ $1+i x-\frac{1}{2} x^{2}$ in the expansion of Eq. (5.17) to arrive at the above expression.

An indirect consequence of the above subtraction procedure of Eq. (5.18) is that the imaginary part is shifted by -1 at large $\alpha_{R}(s)$ and small $\alpha_{I}(s)$ (see Fig. 5.9b). This displacement reflects the coupling between the different materializations of a trajectory and indicates that the removal of a pole has implications for the entire trajectory. A disadvantage of this displacement is the loss of the correct Breit-Wigner behavior at the poles. Therefore, we have opted to correct for this (unphysical) effect by adding the following term to the phase factor in Eq. (5.17):

$$
\zeta_{t} \sin (\pi \alpha(t)) \frac{\left[\pi \alpha_{R}^{\prime}\left(s-m_{p}^{2}\right)\right]^{2}}{e^{\pi \alpha_{I}(s)}+\left[\pi \alpha_{R}^{\prime}\left(s-m_{p}^{2}\right)\right]^{2}} .
$$


The effect of this modification can be appreciated by comparing Fig. $5.9 \mathrm{~b}$ with Fig. 5.9c. Indeed, with the prescription of Eq. (5.20), the Breit-Wigner shape around the poles is restored.

Inspecting Fig. 5.8, it is clear that there is a whole series of $\mathrm{N}^{*}$ and $\Delta^{*} \mathrm{~s}$-channel trajectories. It is not a trivial exercise to determine which ones to include as $\alpha(s)$ trajectories in the duality correction term when performing $\mathrm{p}(\gamma, \mathrm{K}) \mathrm{Y}$ calculations with the Veneziano extension to the Regge model. In the light of the duality hypothesis, it could be argued that every trajectory in the t-channel should have its counterpart in the s-channel. This would provide a selection criterion for the $\alpha(s)$ trajectories. It is not clear, though, how this "duality connection" between the sand $t$-channel trajectories manifests itself. On the basis of gauge invariance, it can be argued that such a connection exists between the kaon and the nucleon trajectory. They both have to be included to obey charge conservation. For the $\mathrm{K}^{*}$ $\mathrm{t}$-channel trajectory, such a reasoning is not readily available and it is not clear how to determine its s-channel counterpart. Therefore we have chosen to include the most dominant nucleon trajectory with unnatural parity, namely the $\mathrm{D}_{15}(1675)$ trajectory. For the K $\Sigma$ production channels, also $\Delta^{*}$ trajectories have to be taken into account. Candidates are the $\mathrm{P}_{33}(1232)$ and the $\mathrm{D}_{33}(1700)$ trajectories.

The corrections produced by the Veneziano duality term on the total $p\left(\gamma, K^{+}\right) \Lambda$ and $p\left(\gamma, \mathrm{K}^{+}\right) \Sigma^{0}$ cross section are illustrated in Fig. 5.10. Obviously, typical s-channel resonance contributions, superimposed on top of the original Regge curves, appear. However, the corrections as obtained within this formalism do not reproduce the data very well. For the $\mathrm{K}^{+} \wedge$ channel, the resonance contributions are far too pronounced and do not improve the original Regge description. In the $\mathrm{K}^{+} \Sigma^{0}$ channel, on the other hand, the Veneziano extension seems to account for more realistic corrections, although also here the results are not fully matching the total cross section data. It has to be stressed that the results are very sensitive to the choices made regarding the relative signs between the various $\alpha(s)$ trajectories. As mentioned in Sec. 5.1, the different trajectories of defined signature receive an additional sign $\eta_{\zeta}$ which remains undetermined in Regge theory. Calculations with various combinations for those relative signs of the trajectories were performed. The results of two combinations are presented in Fig. 5.10, and the adopted $\eta_{\zeta}$ values are summarized in Table 5.2. 

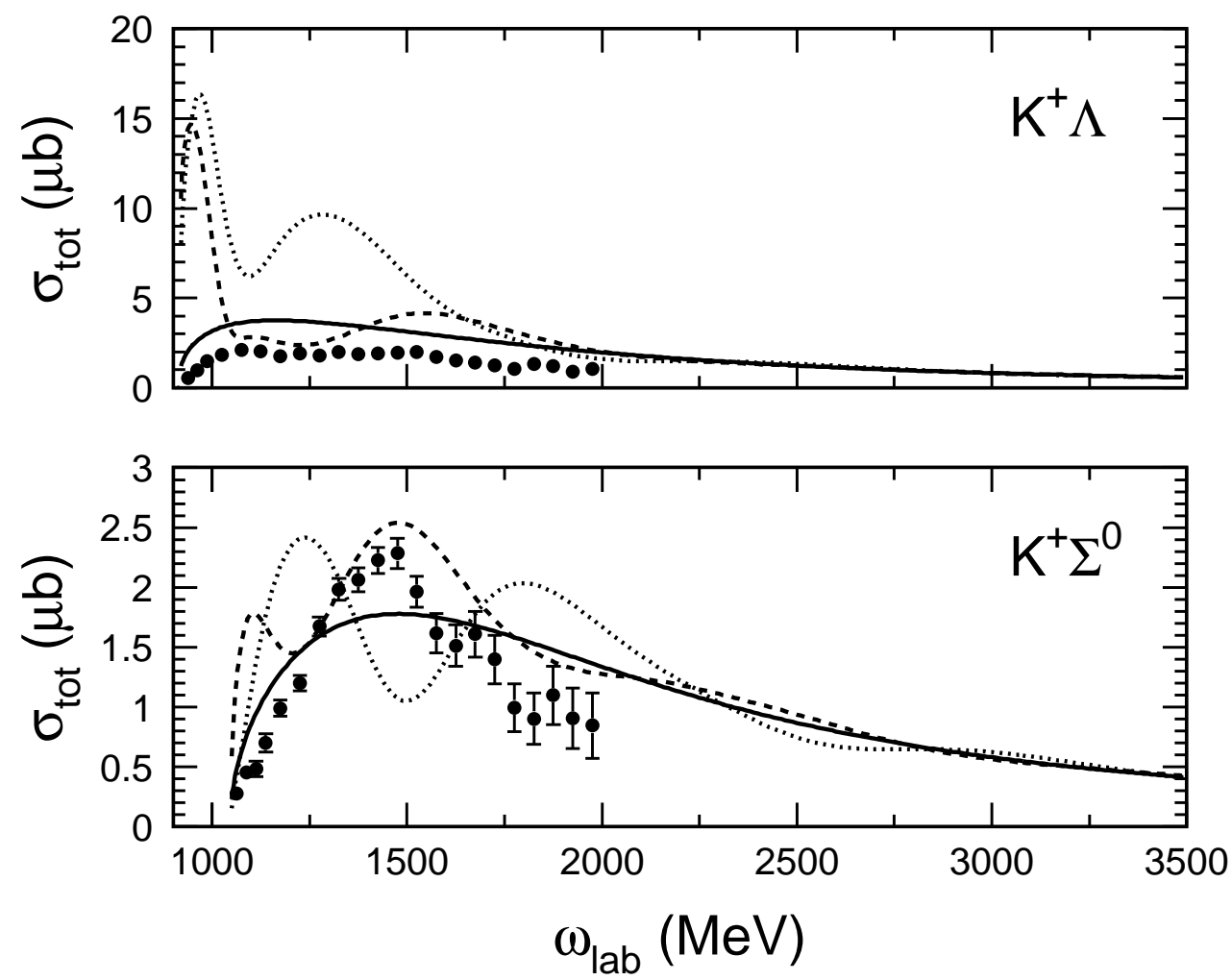

Figure 5.10 Total cross section for the $p\left(\gamma, \mathrm{K}^{+}\right) \Lambda$ and $p\left(\gamma, \mathrm{K}^{+}\right) \Sigma^{0}$ process. The solid line is the Regge model calculation. The dashed and dotted curves are results after including the duality correction term of Eq. (5.12). They differ by the choices with respect to the relative signs $\left(\eta_{\zeta}\right)$ between the various $\alpha(s)$ contributions. The $\eta_{\zeta}$ factors used in those calculations are summarized in Table 5.2. 


\begin{tabular}{||c|cccc||}
\hline \hline$\eta_{\zeta}$ & $N^{*}(939)$ & $N^{*}(1520)$ & $N^{*}(1675)$ & $N^{*}(1990)$ \\
\hline $\begin{array}{r}\text { dashed line: } \\
\text { dotted line: }\end{array}$ & + & + & - & + \\
\hline \hline & - & - & - & - \\
& $\Delta^{*}(1232)$ & $\Delta^{*}(1700)$ & $\Delta^{*}(1905)$ & $\Delta^{*}(1930)$ \\
\hline $\begin{array}{r}\text { dashed line: } \\
\text { dotted line: }\end{array}$ & - & + & + & + \\
\hline \hline
\end{tabular}

Table 5.2 The relative signs $\eta_{\zeta}$ of the $\alpha(s)$ trajectories which are used in the calculations of Fig. 5.10.

To conclude this section, one can state that the duality correction term, inspired by the Veneziano model, is able to introduce "resonant structure" in a Regge model calculation for $p(\gamma, \mathrm{K}) \mathrm{Y}$ reactions. The corrections are effective in the appropriate resonance region and do not alter the Regge model calculations at the higher energies where the coupling constants are determined and where there is no obvious need to introduce extra resonant structures in the predictions. The s-channel corrections in the $\mathrm{K}^{+} \Sigma^{0}$ channel appear more realistic than those in the $\mathrm{K}^{+} \Lambda$ case. For the latter channel, the corrections stemming from s-channel resonances are far too large.

\subsubsection{Beyond the Regge Limit}

The derivation of the Regge amplitude in App. $\mathrm{C}$ and the identification of a Regge propagator in Sec. 5.1 were done in the so called Regge limit ( $s \gg$ and $|t| \ll)$. So, strictly speaking, the expressions are only valid in this regime. As shown in Fig. 5.2, though, reasonable predictions for the $K Y$ production data are obtained at values of - $t$ as large as $1.5 \mathrm{GeV}^{2}$. It is questionable if such a magnitude of $|t|$ still can be considered as sufficiently small. In this section, we report results of exploratory studies which aim at figuring out the validity and possible ways of extending Regge the- 


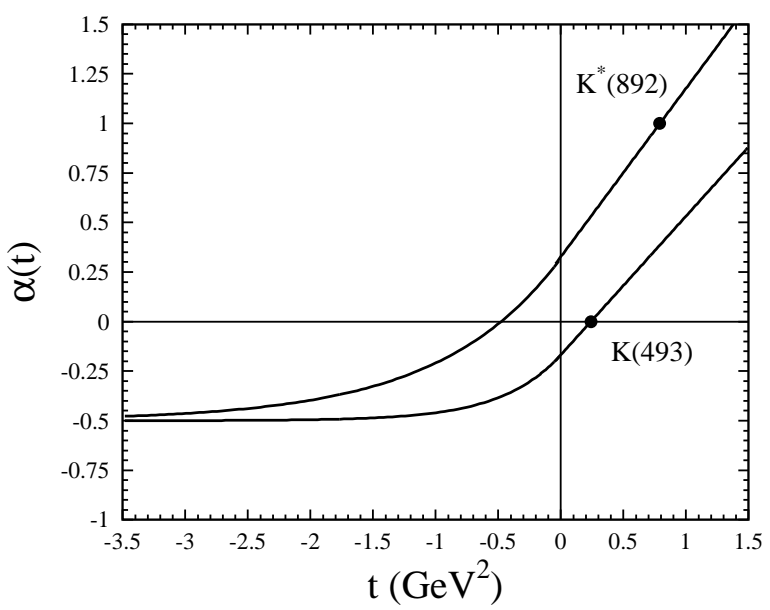

Figure 5.11 The kaon and $K^{*}$ trajectories as a function of $t$. For large negative $t$, the trajectories are saturating at -0.5 . An exponential function is used in the $t \leq 0$ region.

ory for describing $\mathrm{p}(\gamma, \mathrm{K}) \mathrm{Y}$ processes beyond the strict boundaries imposed by the high $s$ and low $|t|$ limit.

A first feature, which appears not properly implemented in the results of Sec. 5.2, is that there are strong indications that the $\alpha(t)$ trajectories should saturate in the limit $t \rightarrow-\infty$. On the basis of counting rules of a constituent inter-exchange model one can prove that $\alpha(t)$ cannot be a linear function at large negative $t$ but, to the contrary, saturates at a finite value $[85,86]$. Such a saturation of the trajectories is also predicted by Sergeenko on the basis of an inter-quark potential [87]. This idea is made clear in Fig. 5.11 for trajectories tending toward a value of -0.5. Implementing an exponential saturation in the model calculations, it is immediately clear that the results are extremely sensitive to the saturation value. Indeed, in Fig. 5.12 some predictions for various choices for the saturation value are displayed. On the basis of these curves, one can conclude that a satisfactory description of the $t$ dependence of the cross section can only be achieved with saturation values smaller than -2. For smaller (absolute) saturation values, the calculated strength largely overshoots the data for $-\mathrm{t} \geq 0.5 \mathrm{GeV}^{2}$. It can be argued that also the introduction of hadronic form factors could help in reducing the cross sections at higher - $t$ [79]. This option is not further explored in this work.

We now wish to determine the range of validity in the variable t of the reggeized 

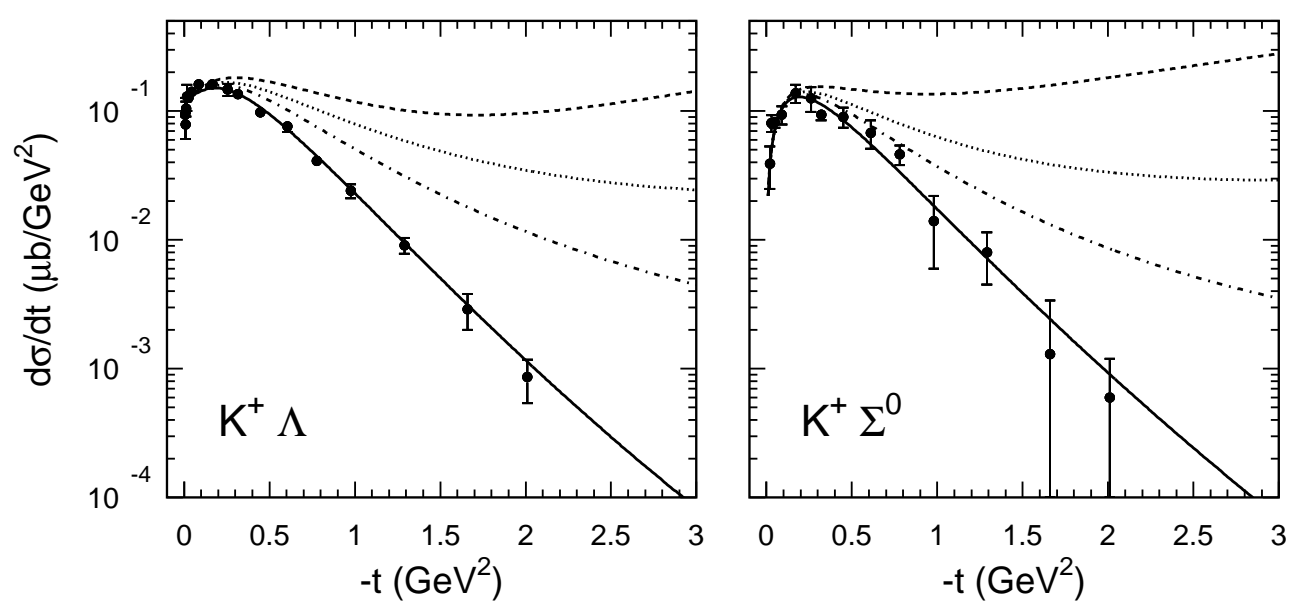

Figure 5.12 The t-dependence of the differential cross section in a Regge model calculation. The solid line is obtained with a linear trajectory. The dashed, dotted and dot-dashed curves are the results of saturating trajectories at $-0.5,-1$ and -2 , respectively. The photon lab energy is $8 \mathrm{GeV}$ and the data are from Ref. [80]. An analogous qualitative behavior is observed at other photon energies.

amplitude of Eq. (5.5). In deriving the latter equation, use is made of the following expression for the asymptotic behavior of the Legendre function:

$$
P_{\alpha}(z) \stackrel{z \rightarrow \infty}{\longrightarrow} \begin{cases}\frac{1}{\sqrt{\pi}} \frac{\Gamma(\alpha+1 / 2)}{\Gamma(\alpha+1)}(2 z)^{\alpha}, & \operatorname{Re}\{\alpha\} \geq-\frac{1}{2}, \\ \frac{1}{\sqrt{\pi}} \frac{\Gamma(-\alpha-1 / 2)}{\Gamma(-\alpha)}(2 z)^{-\alpha-1}, & \operatorname{Re}\{\alpha\} \leq-\frac{1}{2} .\end{cases}
$$

In the derivation of the Regge amplitude in App. C the expression for $\operatorname{Re}\{\alpha\} \geq-\frac{1}{2}$ is used. It is this asymptotic proportionality of $z^{\alpha}$ that eventually gives rise to the Regge behavior for the energy dependence of the amplitude:

$$
M_{\text {Regge }} \sim s^{\alpha(t)}
$$

As was illustrated in Fig. 5.2, this functional dependence on the variables $s$ and $t$ clearly resembles the experimental observations. According to Eq. (5.21), on the other hand, a proportionality factor of the type $z^{-\alpha-1}$ makes its appearance when $\alpha(t) \leq-\frac{1}{2}$, resulting in an amplitude with the following energy dependence.

$$
M_{\text {Regge }} \sim s^{-\alpha(t)-1}
$$


It is obvious that this amplitude diverges in $s$ for $\alpha(t)<-1$. So, on the basis of a mathematically correct implementation of the asymptotic form of the Legendre function, one may exclude the option that the trajectories saturate at values smaller than -1 . On the basis of the findings of Fig. 5.12, however, trajectories saturating at large negative values seem highly preferable.

This is an apparent contradiction which deserves to be better studied. First we wish to point out how the Legendre function exactly appears in the expression for the Regge amplitude. Referring to the derivations of App. C, it turns out that the Legendre function in the Regge amplitude of Eq. (5.5) is approximated by:

$$
P_{\alpha(t)}\left(-\cos \theta_{t}\right) \longrightarrow\left(\frac{s}{s_{0}}\right)^{\alpha(t)} \frac{1}{\Gamma(1+\alpha(t))} .
$$

This expression is based on the asymptotic form of Eq. (5.21) and is a fairly good approximation for the Legendre function as long as $\alpha(t)$ is close to zero. In practice, this corresponds with $t$ values which make $\alpha(t)$ to reside in the vicinity of the pole $\alpha_{0}$. For the kaon trajectory, for example, this pole emerges at $t=+0.24 \mathrm{GeV}^{2}$.

In order to appreciate the impact of the various asymptotic forms for the Legendre function, we have collected in Fig. 5.13 the exact Legendre function and the two approximations of Eqs. (5.21) and (5.24). The panels (a), (b) and (c) correspond to a saturating trajectory at $-0.5,-1$ and a linear trajectory, respectively. First, it seems that the asymptotic form (5.21) depicted by the dashed line describes well the exact Legendre function (solid line), as long as $\alpha(t)$ does not approach -0.5. At this value, the gamma function $\Gamma(\alpha(t)+1 / 2)$ has a pole. In the asymptotic form of Eq. (5.24), the divergence from the function $\Gamma(\alpha(t)+1 / 2)$ has been artificially removed. This can be inspected from the dotted curves of Fig. 5.13. However, this figure also makes clear that the expression (5.24) has a t-dependence which strongly differs form the exact Legendre function when $\alpha(t)$ departs from the $\alpha_{0}$ pole. In Fig. 5.13c where a linear trajectory is used, the deviation is the most severe. Note that the dotted line of this panel corresponds exactly with the form used to obtain the numerical results in Sec. 5.2. From the panels (a) and (b) of this figure, obtained with a saturating $\alpha(t)$, one can conclude that the expression of Eq. (5.24) produces by far smaller values than the exact Legendre function.

From the above discussion of the behavior of the Legendre functions and its asymptotic approximations, one can conclude that in many cases the Regge am- 

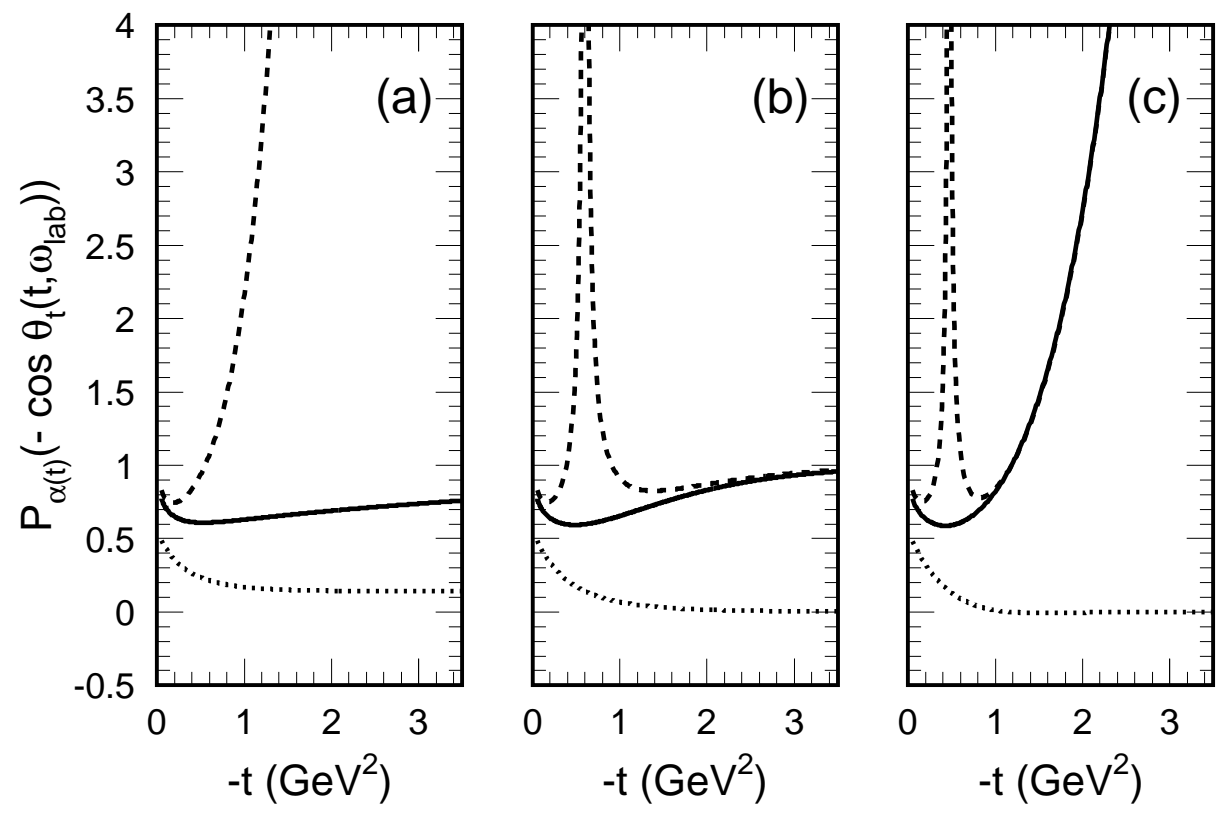

Figure 5.13 The t-dependence of the Legendre function (solid line) and its asymptotic forms as given in Eq. (5.21) (dashed line) and Eq. (5.24) (dotted curve). The latter one is used in the Regge amplitude of Eq. (5.5). Panel (a) contains a saturating trajectory at a value of -0.5 whereas the trajectory of panel (b) saturates at -1 . For panel (c), a linear trajectory is used. The photon lab energy is $8 \mathrm{GeV}$. Similar behavior is observed for other photon energies.

plitude of Eq. (5.5) is used under physical conditions extending the strict range of applicability of the mathematical formula which lies behind its derivation. Only for very small values of $\alpha(t)$, the approximation of Eq. (5.24) and the proportionality factor $s^{\alpha(t)}$ can be mathematically justified. For $\alpha(t)$ values corresponding to $\mathrm{t} \approx 0$, the approximation (5.24) becomes poorer and for $\alpha(\mathrm{t})$ values beyond -0.5 , a behavior as $s^{-\alpha(t)-1}$ is predicted by the exact Legendre function.

In view of the above restrictions, we have made an attempt to derive a modified Regge propagator which is based on an exact expression for the Legendre functions. Starting from Eq. (C.32) and along the same lines of App. C and Sec. 5.1, one can construct a modified propagator $\widetilde{\mathcal{P}}_{\text {Regge }}$ which has the following form:

$$
\widetilde{\mathcal{P}}_{\text {Regge }}^{\zeta= \pm}(s, t)=-(2 \alpha(t)+1) \frac{\pi \alpha^{\prime}}{\sin (\pi \alpha(t))} \frac{1+\zeta e^{-i \pi \alpha(t)}}{2} P_{\alpha(t)}\left(-\cos \theta_{t}\right)
$$



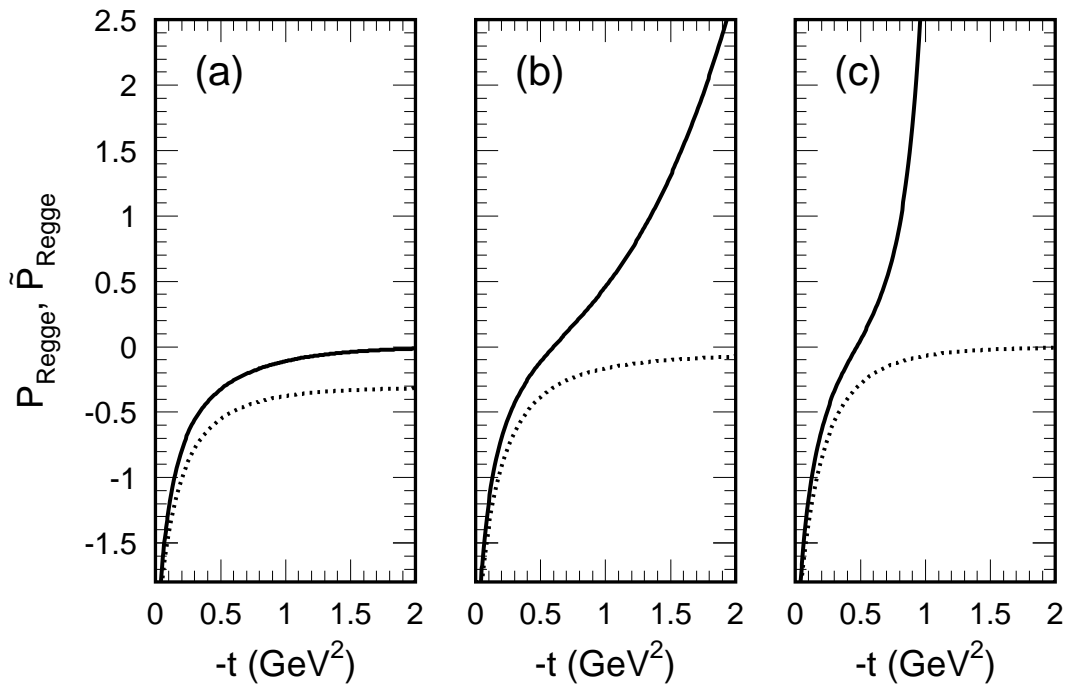

Figure 5.14 The $t$-dependence of the propagators $\widetilde{\mathcal{P}}_{\text {Regge }}$ (solid line) and $\mathcal{P}_{\text {Regge }}$ (dotted line) as defined in the Eqs. (5.25) and (5.6), respectively. The phase factor $[1+\zeta \exp (-i \pi \alpha(t))] / 2$ is not included. The different panels use $\alpha(t)$ trajectories as in Fig. 5.13. The photon lab energy is $8 \mathrm{GeV}$.

It can be easily proven that in the limit $\alpha(t) \rightarrow 0, \widetilde{\mathcal{P}}_{\text {Regge }}(s, t)$ is identical to the original and commonly adopted propagator $\mathcal{P}_{\text {Regge }}(s, t)$ of Eq. (5.6). Both the original and modified Regge propagator proposed here, are displayed in Fig. 5.14 for a linear trajectory (c), and a trajectory saturating at -0.5 (a) and -1 (b). Inspecting this figure, it is observed that in all cases $\mathcal{P}_{\text {Regge }}$ Converges at larger values of $-t$, due to the proportionality $s^{\alpha(t)}$. The modified Regge propagator, on the other hand, diverges at larger values of $-t$. An exception should be made for the case where the trajectory is made to converge at the value -0.5 . This peculiar behavior can be attributed to the presence of the factor $[2 \alpha(t)+1]$ in Eq. (5.25) and the convergence of the other factors. In panel (b), where $\alpha(t) \rightarrow-1$, the Legendre function adopts a value of about 1 (Fig. 5.13b). However, in this particular case, the divergence of $\widetilde{\mathrm{P}}_{\text {Regge }}$ is due to the pole produced by $[\sin (\pi \alpha(\mathrm{t}))]^{-1}$. In panel (c), the steep raise is a combination of the $\sin (\pi \alpha(t))$ pole at $\alpha(t)=-1$ and the diverging Legendre function at large $t$ (Fig. 5.13c).

From the above investigations of the modified propagator $\widetilde{\mathcal{P}}_{\text {Regge }}$ it seems that only a trajectory saturating at a value approaching - 0.5 can produce acceptable re- 

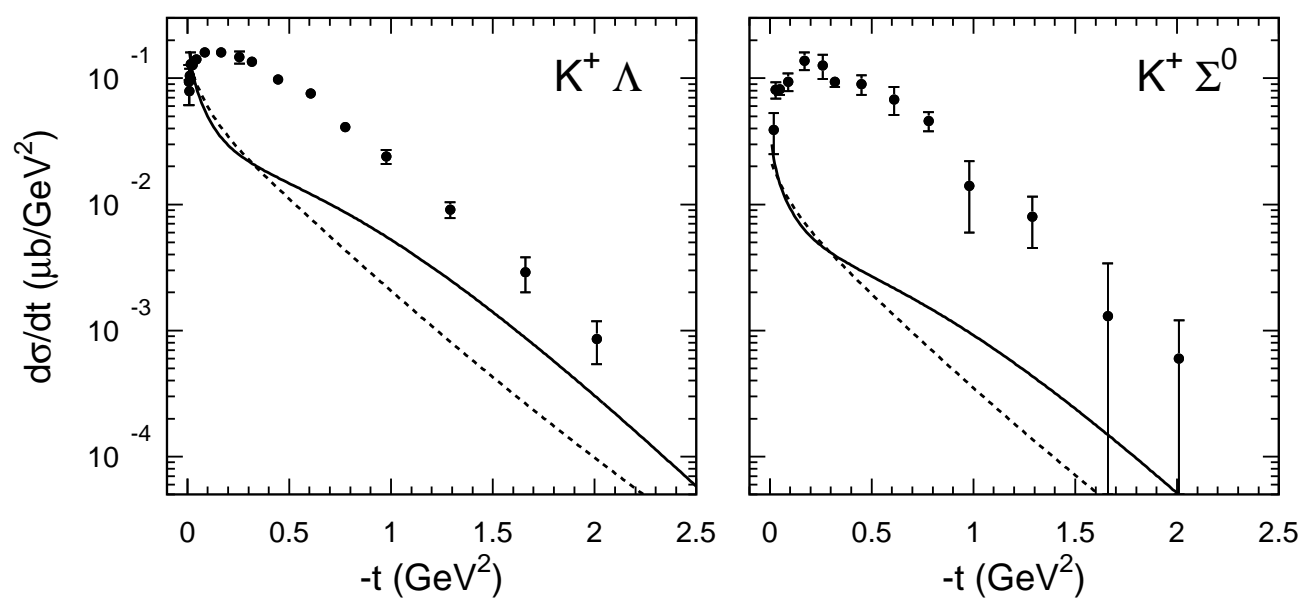

Figure 5.15 Contribution of the kaon trajectory to the differential $p\left(\gamma, \mathrm{K}^{+}\right) \wedge$ and $p\left(\gamma, \mathrm{K}^{+}\right) \Sigma^{0}$ cross section. The solid line is obtained with the modified $\widetilde{\mathcal{P}}_{\text {Regge }}$ propagator of Eq. (5.25) and a saturating trajectory at -0.5 . The dashed line is the result of a calculation with the original $\mathcal{P}_{\text {Regge }}$ propagator of Eq. (5.6) and a linear trajectory. The photon lab energy is 8 $\mathrm{GeV}$ and the data are from Ref. [80].

sults which are free from divergences. Right at the start of this section, it was already mentioned that there are strong arguments which speak in favor of trajectories $\alpha(t)$ saturating at large negative values of $t$. In Fig. 5.15 we have plotted the amplitude produced by a saturating kaon trajectory at -0.5 , reggeized with the $\widetilde{\mathcal{P}}_{\text {Reg gepropagator. In addition, the kaon strength obtained with the original }} \mathcal{P}_{\text {Regge }}$ and a linear trajectory is shown. It seems that the variations in the predictions of both approaches are not spectacular. This feature is not that surprising given the $\mathrm{t}$-dependence of the propagators in Fig. 5.14. The solid line of panel (a) and the dotted line of panel (c) are relatively close.

As mentioned in Sec. 5.2 and exemplified in Fig. 5.2, in a Regge model the largest fraction of the $\mathrm{p}\left(\gamma, \mathrm{K}^{+}\right) \mathrm{Y}$ strength can be attributed to the exchange amplitude stemming from the $\mathrm{K}^{*}$ trajectory. However, a difficulty emerges with the $\mathrm{K}^{*}$ trajectory when introducing a modified Regge propagator as it was written in Eq. (5.25). A K* trajectory which saturates at a value of -0.5 is physically acceptable but in order to force the amplitude to match the corresponding Feynman expression at the $\alpha_{0}$ pole, the $K^{*}$ trajectory receives an offset of -1 according to the spin shift of Eq. (5.7). This results in an effective saturation value of -1.5 that enters 

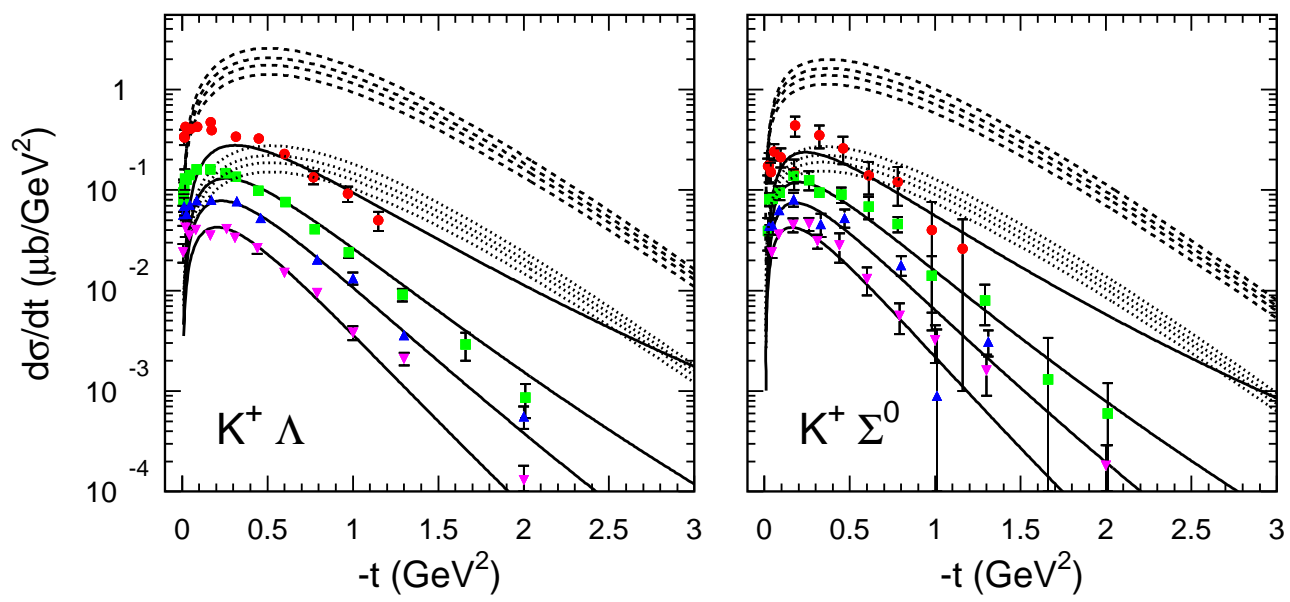

Figure 5.16 Contribution of the $\mathrm{K}^{*}$ trajectory to the differential $p\left(\gamma, \mathrm{K}^{+}\right) \Lambda$ and $p\left(\gamma, \mathrm{K}^{+}\right) \Sigma^{0}$ cross section. The solid lines are obtained with the original $\mathcal{P}_{\text {Regge }}$ propagator and a linear trajectory. The dashed lines are the result of a calculation with the modified $\widetilde{\mathcal{P}}_{\text {Regge }}$ propagator and a saturating trajectory at +0.5 . For the dotted curves, the coupling constants of the dashed-line model were adjusted. The data are as in Fig. 5.2.

the calculations. From Eq. (5.21), it is obvious that this inevitably gives rise to a diverging Legendre function and an unphysical amplitude.

A possible way out may be the following. If the $\mathrm{K}^{*}$ trajectory is made to saturate at +0.5 , the "spin shift" of -1 will force the saturation to occur at an effective value of -0.5. To our knowledge, however, there are no physical arguments for introducing a positive saturation value. Anyway, we have plotted the result for this option in Fig. 5.16. It turns out that the exchange of the $\mathrm{K}^{*}$ trajectory, calculated with the

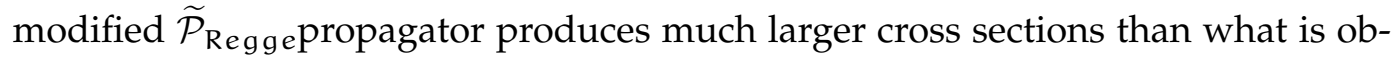
tained with the original Regge propagator. However, the values for the two $\mathrm{K}^{*}$ coupling constants were adjusted to the data and can in principle be refitted. By doing so, the numerical calculations can be made to reproduce the right order of magnitude of the cross sections but the s-dependence of the amplitude seems not to correspond to what is experimentally observed. The difference in the computed strength for the 5, 8, 11 and $16 \mathrm{GeV}$ photon lab energies is too small, pointing to problems with the s-dependence of the used propagator. It should be stressed that this failure of the s-dependence is not observed in similar calculations for the kaon trajectory although this is not explicitly made clear in Fig. 5.15. For the kaon am- 
plitudes, the correct separations in s are revealed.

The reason for this different energy behavior of the two trajectory exchanges has to be sought in a different energy dependence of the corresponding vertex functions. For the kaon coupling, the vertex function is almost independent on the energy whereas this is not the case for the vertex function of the $\mathrm{K}^{*}$ particle. Since

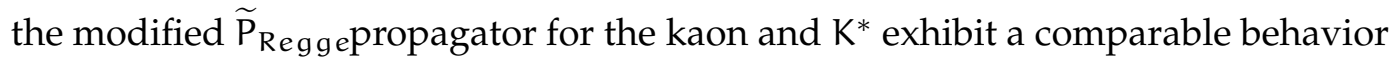
(they both have a $\alpha(t)$ that saturates at an effective value of -0.5), the differences in the energy dependence of the vertex function is revealed in amplitudes. The original Regge propagator, calculated with a linear trajectory, behaves like:

$$
\begin{aligned}
& \mathrm{P}_{\text {Regge }}^{\mathrm{K}} \sim \mathrm{s}^{\alpha_{K}(t)}=\mathrm{s}^{0.70 \mathrm{t}-0.17}, \\
& \mathrm{P}_{\text {Regge }}^{\mathrm{K}^{*}} \sim \mathrm{s}^{\alpha_{\mathrm{K} *}(\mathrm{t})-1}=\mathrm{s}^{0.85 \mathrm{t}-0.68} .
\end{aligned}
$$

This difference in powers of $s$ seems to absorb the s-dependence of the vertex functions and results in an energy dependence of the amplitude in accordance with the experiment.

The bottom line of the above discussion is that the original form of the Regge amplitude can be successfully applied over a relatively wide area in the $(s, t)$ Mandelstam plane. This area in $(s, t)$ greatly outreaches the range of applicability which one could expect on rigorous mathematical grounds. Straight extensions of the theory based on seemingly more rigid mathematical expressions appear to curtail the general applicability of Regge theory. In that respect, Regge theory appears to have a considerable phenomenological basis.

To conclude this subsection, we wish to stress that we are aware of the fact that the material presented here raises more questions than it actually answers. We are hopeful, though, that the considerations presented here may eventually contribute to a more profound understanding of the successes and limitations of Regge theory and to its possible extension into the resonance region. 



\section{Chapter 6}

\section{Conclusion and Outlook}

In this work, we investigated the open-strangeness production off the proton induced by real and virtual photons. To be more specific, we addressed the issue of describing and interpreting $\mathrm{p}(\gamma, \mathrm{K}) \mathrm{Y}$ and $\mathrm{p}\left(e, e^{\prime} \mathrm{k}\right) \mathrm{Y}$ reactions in a (virtual) photon energy range which extends from threshold up to a few $\mathrm{GeV}$. We have adopted two fairly complementary techniques based on different ways of assessing the underlying degrees-of-freedom. In the isobar model, the basic degrees-of-freedom are the hadrons and their excited states and they are described in terms of effective fields. In the Regge model, the description does not take into account individual particles but rather goes out from properties of classes of hadronic states, called trajectories, determined by partonic degrees-of-freedom. The two descriptions provide a complementary picture of the problem under investigation.

In the isobar model, the kaon production reactions are studied in terms of hadronic degrees-of-freedom employing a field-theoretic approach. We have reached a fair description of the available SAPHIR database for the three reaction channels

$\gamma p \rightarrow \mathrm{K}^{+} \Lambda, \mathrm{K}^{+} \Sigma^{0}$ and $\mathrm{K}^{0} \Sigma^{+}$. Model calculations are presented for the total and differential cross sections as well as for the recoil polarization asymmetries for all three channels. In addition, predictions are made for observables for which there are at present no data available. Such an observable, for example, is the photon beam asymmetry.

From our model calculations for the $\mathrm{K}^{+} \wedge$ channel, the data seems to support evidence for a significant role of the four nucleon resonances $S_{11}(1650), P_{11}(1710)$, $\mathrm{P}_{13}(1720)$ and $\mathrm{D}_{13}(1895)$. For the $K \Sigma$ processes, we were able to identify a set of 
three $N^{*}$ resonances $\left(S_{11}(1650), P_{11}(1710), P_{13}(1720)\right)$ and two $\Delta^{*}$ states $\left(S_{31}(1900)\right.$ and $\mathrm{P}_{31}(1910)$ ), with which a satisfactory description of the data can be obtained.

In the search for the dominant resonant contributions, the issue of the "missing resonances" was thoroughly addressed. In the $\mathrm{K}^{+} \wedge$ channel, there is clearly some need for an additional, formerly unobserved, resonance in the mass range about $1.9 \mathrm{GeV}$. We find that the overall description of the data is significantly improved after introducing a $\mathrm{D}_{13}(1895)$ state, as was recently also suggested by the George Washington group. However, in this work it is shown that also alternative resonances with other quantum numbers could account for the observed "structure" in the energy dependence of the data. In that respect, it is stressed that the identification of the quantum numbers of this new state and a further characterization have to be addressed with the greatest care. With no doubt, further confirmation in other observables is necessary to pin down the exact nature of this missing nucleon resonance. A possibility is also to look for indications of this missing resonance in other isospin channels. In $K \Sigma$ photoproduction reactions, our calculations do not provide evidence for a salient role for the missing $\mathrm{D}_{13}(1895)$ resonance.

From the investigations presented in this work, it becomes clear that the treatment of background processes in the $p(\gamma, \mathrm{K}) \mathrm{Y}$ reactions is not free of ambiguities. We have shown that the "bare" Born amplitudes, which are constrained on the basis of SU(3)-flavor symmetry, produce cross sections which dramatically overshoot the measured ones. Therefore, additional ingredients in the model calculations beyond resonance contributions appear essential. We have presented three different schemes which accomplish to cut down satisfactorily the magnitude of the Born amplitudes. They are based on the introduction of soft hadronic form factors for the Born terms (model A), the introduction of hyperon resonances in the $u$-channel (model B) or the presumption of a very strong SU(3)-flavor symmetry breaking at the level of the coupling constants (model C). With the existing database, it is impossible to decide on empirical grounds which one of the three approaches appears favorable. All three schemes succeed in reproducing the photoproduction data fairly well. However, through the background diagrams, some model dependence in the extracted resonance parameters gets introduced. This dependence turns out to be small for the $\Delta^{*}$ particles. For some of the $\mathrm{N}^{*}$ resonances, though, the extracted coupling constants may vary up to an order of magnitude, depend- 
ing on which model is used to implement the background. Predictions for some of the unmeasured observables also exhibit these model dependences. Calculations for the $p\left(\gamma, \mathrm{K}^{+}\right) \Sigma^{0}$ photon beam asymmetry are only moderately sensitive to the implementation of the background terms. This is not the case for the photon beam asymmetries in the $\mathrm{p}\left(\gamma, \mathrm{K}^{+}\right) \Lambda$ and $\mathrm{p}\left(\gamma, \mathrm{K}^{0}\right) \Sigma^{+}$channel. Similar variations are also observed in double polarization asymmetries.

Closely related to the treatment of the background, we have investigated how sensitive the predictions are to the adopted recipes for the phenomenological hadronic form factor $\widehat{F}$, appearing in the contact terms which are introduced to conserve gauge invariance. Former investigations mainly used the Ohta or Haberzettl prescription for the form factor $\widehat{F}$. Recently, Davidson and Workman pointed out that both of these recipes are theoretically unacceptable and provided an alternate form. A systematic study on the consequences of these corrections for the computed $p\left(\gamma, \mathrm{K}^{+}\right) \wedge$ observables has been made. In the energy range under investigation, the corrections are rather large and the effects on the extracted coupling constants is substantial.

Apart form the photoproduction processes, the electroinduced $p\left(e, e^{\prime} \mathrm{K}\right) \mathrm{Y}$ reactions are addressed. The current database is small and a reliable extraction of $\mathrm{Q}^{2}$ dependent resonance parameters remains unfeasible at present. The recent JLab data for the longitudinal $\mathrm{K}^{+} \wedge$ response, a quantity which is not probed by the real photons, seems to have the potential to discriminate between the different background models which are constructed to describe the photoproduction data. The schemes introducing soft hadronic form factors or strong SU(3)-flavor symmetry breaking seem not to be favored by the electroproduction data. Such strong variations between the different model predictions are not observed in the $\mathrm{K}^{+} \Sigma^{0}$ channel. At present, however, no data is available for the separated $p\left(e, e^{\prime} K^{0}\right) \Sigma^{+}$response functions. After all, the longitudinal component of the electroinduced process seems to be a powerful tool to further constrain the reaction dynamics and will likely have its impact on the models constructed on the basis of photoproduction.

Summarizing, it is clear that even an extensive and accurate data base as the one produced by the SAPHIR collaboration, does not allow to determine unambiguously the various contributions to the $p(\gamma, \mathrm{K}) \mathrm{Y}$ reaction dynamics. The measured cross sections and recoil polarization asymmetries do not suffice to fully nail down 
the complicated interference pattern between the various contribution. This is not very surprising given that a complete meson photoproduction experiment needs at least seven observables to fully constrain the reaction amplitude at a fixed photon energy [57].

In the foreseeable future, however, the available amount of strangeness production data in the resonance region will dramatically increase. Research efforts at JLab [88], GRAAL (Grenoble) [59] and SPring-8 [60] and continuing analysis work from the SAPHIR collaboration [55] will substantially extend the amount of $\Lambda$ and $\Sigma$ photo- and electroproduction data and will shed light on the capability or incapability of hadronic approaches to model the physics at higher photon energies. They will also provide large and accurate sets of polarization data. With such an extensive data base at hand, one can be hopeful to better constrain the theoretical models, reveal the full dynamics of strangeness production reactions and extract reliable information regarding the mass position and various decay widths of the resonances.

In the second part of this work, a Regge theory based model is applied to describe the same strangeness photo- and electroproduction data. In literature, it was shown that such a model is able to excellently account for the high energy $p(\gamma, \mathrm{K}) \mathrm{Y}$ data at forward angles. In a Regge based model description, very few parameters are required and they can all be fixed by comparing the model calculations to the data above the resonance region. In this work, we have made an attempt to extend the Regge model to the lower energies and apply it in the resonance region. We have shown that even without introducing additional ingredients in the theory or without modifying the parameters, the Regge based model succeeds in providing a fair description of the data in the resonance region. For example the correct signs and magnitudes in the observed asymmetry observables for both the $\mathrm{K}^{+} \Lambda$ and $\mathrm{K}^{+} \Sigma^{0}$ channels are reproduced in a natural way. On the other hand, the structure in the observables, usually attributed to the occurrence of s-channel resonances, cannot be accounted for in a Regge based model.

In an attempt to add the physics of the $\mathrm{N}^{*}$ and $\Delta^{*}$ resonances to a Regge based description, a correction term, based on the Veneziano model, is introduced. This correction term takes into account the duality hypothesis and avoids double count- 
ing of s- and t-channel poles. It is shown that the correction term introduces resonant structures in the observables. For the $\mathrm{K}^{+} \Sigma^{0}$ channel, the results are in reasonable agreement with what is experimentally observed. In the case of the $\mathrm{K}^{+} \Lambda$ process, however, the resonance corrections of the Veneziano model are far too pronounced.

Further, we have explored the possibilities to apply Regge inspired models beyond the so called Regge domain of large $s$ and small $t(s \gg$ and $|t| \ll)$. The Regge amplitude is derived under those strict conditions and is in principle only applicable in this limited Regge domain. Surprisingly, a Regge inspired model appears able to account for the $p(\gamma, \mathrm{K}) \mathrm{Y}$ data in a wide kinematical domain which largely exceeds the strict Regge domain determined by the conditions $s \gg$ and $|t| \ll$. We have made an attempt to formulate possible modifications to the Regge amplitude which could make it theoretically more acceptable in this wider kinematical range. In doing so, we came across a number of technical and mathematical difficulties for which no clear-cut solutions could be put forward. As a matter of fact, it appears that both conceptually and technically the extension of the powerful ideas of Regge theory into the resonance region is far from straightforward and more theoretical efforts are clearly needed. 



\section{Appendix A}

\section{Effective Field Theory and Pseudo-scalar Meson Production}

In an isobar model, the hadrons and their excited states are treated as individual particles and described by effective fields. In this appendix, the building blocks of such a field theory are presented. The effective Lagrangians used in meson photoproduction calculations are given in numerous works [15, 25, 50, 89]. However, for the sake of defining our notation and normalization conventions, we summarize the ones which are relevant for the "strange" meson production processes. In Sec. A.1, the expressions for the $\mathrm{p}\left(\gamma, \mathrm{K}^{+}\right) \wedge$ process are summarized. In Sec. A.2, we point out how these expressions can be generalized in order to cover the other isospin channels. In the process of describing the electroproduction of hadrons, electromagnetic form factors become an essential ingredient. In Sec. A.3, we list the electromagnetic form factors which are needed for modeling $p\left(e, e^{\prime} \mathrm{K}\right) \mathrm{Y}$ reactions.

\section{A.1 Effective Lagrangians for $p\left(\gamma, \mathrm{K}^{+}\right) \wedge$ Reactions}

\section{A.1.1 Interaction Lagrangians}

The interaction Lagrangians indicate how the electromagnetic and hadronic fields couple to each other. Thereby, the coupling strength at each vertex is expressed by a coupling constant. Since the fields are effective, those coupling constants are not determined by the theory itself, but treated as a parameter.

Hereafter, the interaction Lagrangians are listed according to the type of reso- 
nance exchange.

\section{Born Terms}

The electromagnetic interaction Lagrangians for the Born terms are given by:

$$
\begin{aligned}
\mathcal{L}_{\gamma p p} & =-e \bar{N} \gamma_{\mu} N A^{\mu}+\frac{e \kappa_{p}}{4 m_{p}} \bar{N} \sigma_{\mu \nu} N F^{\mu \nu}, \\
\mathcal{L}_{\gamma \wedge \Lambda} & =\frac{e \kappa_{\Lambda}}{4 m_{p}} \bar{\Lambda} \sigma_{\mu \nu} \Lambda F^{\mu \nu}, \\
\mathcal{L}_{\gamma \wedge \Sigma^{0}} & =\frac{e \kappa_{\Sigma}{ }^{0}}{4 m_{p}} \overline{\Sigma^{0}} \sigma_{\mu \nu} \Lambda F^{\mu \nu}+\text { h.c. } \\
\mathcal{L}_{\gamma K K} & =-i e\left(K^{\dagger} \partial_{\mu} K-K \partial_{\mu} K^{\dagger}\right) A^{\mu} .
\end{aligned}
$$

The electric charge is defined as $e=+\sqrt{4 \pi / 137}$ and the anti-symmetric tensor for the photon field $A^{\mu}$ is defined as $F^{\mu \nu}=\partial^{\nu} A^{\mu}-\partial^{\mu} A^{\nu} . N, \Lambda$ and $K$ represent in those expressions the nucleon, lambda and kaon fields, respectively. For the hadronic $\mathrm{K} \wedge \mathrm{p}$ interaction, a pseudo-scalar (PS) or pseudo-vector (PV) option is viable:

$$
\begin{aligned}
& \mathcal{L}_{\mathrm{K} \wedge p}^{\mathrm{PS}}=-i g_{\mathrm{K} \wedge \mathrm{p}} \mathrm{K}^{\dagger} \bar{\Lambda} \gamma_{5} \mathrm{~N}+\text { h.c. } \\
& \mathcal{L}_{\mathrm{K} \wedge p}^{\mathrm{PV}}=\frac{\mathrm{f}_{\mathrm{K} \wedge \mathrm{p}}}{\mathrm{m}_{\mathrm{K}}} \partial^{\mu} \mathrm{K}^{\dagger} \bar{\Lambda} \gamma_{\mu} \gamma_{5} \mathrm{~N}+\text { h.c. }
\end{aligned}
$$

All results in this work are obtained with the PS variant. For the anomalous magnetic moments we have used the values $\kappa_{p}=1.793, \kappa_{\wedge}=-0.613$ and $\kappa_{\Sigma} \Sigma_{\Lambda}=1.61$ [58].

\section{Spin-1 Meson Exchange}

The electromagnetic coupling to a vector meson (V) is described by:

$$
\mathcal{L}_{\gamma \mathrm{K} V}=\frac{e K_{\mathrm{K} V}}{4 \mathrm{~m}} \epsilon^{\mu \nu \lambda \sigma} \mathrm{F}_{\mu \nu} \mathrm{V}_{\lambda \sigma} \mathrm{K}
$$

where the vector meson tensor is given by $V^{\mu \nu}=\partial^{\nu} V^{\mu}-\partial^{\mu} V^{\nu}$ and $V^{\mu}$ is the vector field. The photon coupling to an axial vector meson $\left(V_{a}\right)$ reads:

$$
\mathcal{L}_{\gamma K V_{a}}=i \frac{e K_{K V_{a}}}{m}\left(\partial_{\mu} A_{\nu} \partial^{\mu} V_{a}^{\nu}-\partial_{\mu} A_{\gamma} \partial^{\nu} V_{a}^{\mu}\right) K
$$

where $V_{a}^{\mu}$ is the axial vector field. The mass scale $m$ for the transition moment is arbitrary chosen as $1.0 \mathrm{GeV}$. The complete anti-symmetric tensor is defined as 
$\epsilon^{0123}=1$ according to the conventions in Ref. [90]. The hadronic vertex has a vector $(v)$ and a tensor $(t)$ part:

$$
\begin{aligned}
\mathcal{L}_{\mathrm{V} \wedge p}= & -g_{V \wedge p}^{\nu} \bar{\Lambda} \Gamma_{\mu} N^{\mu} \\
& +\frac{g_{V \wedge p}^{t}}{2\left(m_{\wedge}+m_{p}\right)} \bar{\Lambda} \sigma_{\mu \nu} V^{\mu \nu} \Gamma N+\text { h.c. }
\end{aligned}
$$

where $V$ is now a short-hand notation for both a vector and an axial vector meson. Furthermore, $\Gamma=1\left(\gamma^{5}\right)$ and $\Gamma^{\mu}=\gamma^{\mu}\left(\gamma^{\mu} \gamma^{5}\right)$ for vector (axial vector) meson resonances. The information about the (axial) vector meson coupling constants which can be extracted from fits to the data is a combination of the electromagnetic and the hadronic coupling. In this work, those values are normalized as:

$$
\begin{aligned}
G_{V}^{v} & =\frac{e g_{V \wedge p}^{v}}{4 \pi} \kappa_{K V}, \\
G_{V}^{t} & =\frac{e g_{V \wedge p}^{t}}{4 \pi} K_{K V},
\end{aligned}
$$

with $\mathrm{V}$ a vector or axial vector meson.

\section{Spin-1/2 Resonance Exchange}

For spin-1/2 resonances, the electromagnetic interaction reads:

$$
\mathcal{L}_{\gamma B R}=\frac{e \kappa_{B R}}{4 m_{p}} \bar{R} \Gamma_{\mu \nu} B+\text { h.c. }
$$

where the hadronic vertices are described by a pseudo-scalar (PS) or a pseudovector (PV) part:

$$
\begin{aligned}
\mathcal{L}_{\mathrm{KBR}}^{\mathrm{PS}} & =-i g_{\mathrm{KBR}} \mathrm{K}^{\dagger} \overline{\mathrm{B}} \Gamma \mathrm{R}+\text { h.c. } \\
\mathcal{L}_{\mathrm{KBR}}^{\mathrm{PV}} & =\frac{f_{\mathrm{KBR}}}{m_{\mathrm{K}}}\left(\partial^{\mu} \mathrm{K}^{\dagger}\right) \overline{\mathrm{B}} \Gamma_{\mu} \mathrm{R}+\text { h.c. }
\end{aligned}
$$

Herein, $\Gamma^{\mu \nu}=\gamma^{5} \sigma^{\mu \nu}\left(\sigma^{\mu \nu}\right)$ for odd (even) parity resonances. $\Gamma$ and $\Gamma^{\mu}$ are defined as before. Further, $\mathrm{B}$ is the baryon field (a $\mathrm{p}$ or $\Lambda$ depending on the corresponding vertex) and $R$ is the spin-1/2 baryon resonance field (a $N^{*}$ or $Y^{*}$ ). In this work we have used the PS scheme for the hadronic coupling. For spin-1/2 resonance exchange, the information regarding the extracted coupling constant takes on the form:

$$
\mathrm{G}_{\mathrm{R}}=\frac{\mathrm{g}_{\mathrm{KBR}}}{\sqrt{4 \pi}} \kappa_{\mathrm{BR}} .
$$




\section{Spin-3/2 Resonance Exchange}

For spin-3/2 resonances, there are two terms in the Lagrangian describing the electromagnetic interaction:

$$
\begin{aligned}
\mathcal{L}_{\gamma B R}= & i \frac{e \kappa_{B R}^{(1)}}{2 m_{p}} \bar{R}^{\mu} \theta_{\mu \nu}(Y) \Gamma_{\lambda} B F^{\lambda \nu} \\
& -\frac{e \kappa_{B R}^{(2)}}{4 m_{p}^{2}} \bar{R}^{\mu} \theta_{\mu \nu}(X) \Gamma\left(\partial_{\lambda} B\right) F^{\nu \lambda}+\text { h.c. }
\end{aligned}
$$

The hadronic vertex is given by:

$$
\mathcal{L}_{\mathrm{KBR}}=\frac{f_{\mathrm{KBR}}}{\mathrm{m}_{\mathrm{K}}} \overline{\mathrm{R}}^{\mu} \theta_{\mu \nu}(\mathrm{Z}) \Gamma^{\prime} \mathrm{B}\left(\partial^{\nu} \mathrm{K}\right)+\text { h.c. . }
$$

Here, $\Gamma$ and $\Gamma^{\mu}$ are defined as above and $\Gamma^{\prime}=\gamma^{5}$ (1) for odd (even) parity resonances. The function $\theta_{\mu \nu}(\mathrm{V})$ reflects the invariance of the free Lagrangian of a spin-3/2 field under a point transformation and is given by [91]:

$$
\theta_{\mu \nu}(V)=g_{\mu \nu}-\left(V+\frac{1}{2}\right) \gamma_{\mu} \gamma_{\nu}
$$

The parameters $V=X, Y, Z$ are the so called off-shell parameters. For spin-3/2 resonance exchange, the fits of the model calculations to the data give access to the following combination of coupling constants:

$$
\begin{aligned}
& G_{R}^{(1)}=\frac{e f_{K B R}}{4 \pi} \kappa_{B R}^{(1)}, \\
& G_{R}^{(2)}=\frac{e f_{K B R}}{4 \pi} K_{B R}^{(2)} .
\end{aligned}
$$

\section{A.1.2 Propagators}

The free Lagrangian of an effective field determines its propagator. For the (pseudo) scalar and the (axial) vector particles, we have adopted the standard expressions:

$$
\begin{aligned}
\mathcal{P}_{0}(q) & =i \frac{1}{q^{2}-m^{2}}, \\
\mathcal{P}_{1}^{\mu v}(q) & =i \frac{1}{q^{2}-m^{2}}\left[-g^{\mu \nu}+\frac{q^{\mu} q^{\nu}}{m^{2}}\right],
\end{aligned}
$$

where $q$ is the transferred momentum and $m$ the mass of the intermediate particle. For spin-1/2 fermions, the propagator reads:

$$
\mathcal{P}_{1 / 2}(q)=i \frac{\not q+m}{q^{2}-m^{2}}
$$


The optimal choice for a spin-3/2 propagator remains a subject of discussion [91]. In this work we have used the Rarita-Schwinger form:

$$
\begin{aligned}
\mathcal{P}_{3 / 2}^{\mu \nu}(q)= & i \frac{\not q+m}{3\left(q^{2}-m^{2}\right)} \\
& \times\left[3 g^{\mu \nu}-\gamma^{\mu} \gamma^{\nu}-\frac{2 q^{\mu} q^{\nu}}{m^{2}}-\frac{\gamma^{\mu} q^{\nu}-\gamma^{\nu} q^{\mu}}{m}\right] .
\end{aligned}
$$

In order to account for the finite lifetime of the resonances, a width $\Gamma$ is included in the propagators of the s-channel particles. This is formally done by the replacement:

$$
\mathrm{q}^{2}-\mathrm{m}^{2} \longrightarrow \mathrm{q}^{2}-\mathrm{m}^{2}+\mathrm{im} \Gamma
$$

It should be stressed that this procedure is not fully compatible with the request of unitarity for the total scattering amplitude [91]. However, a unitarization procedure as performed for $\pi$ production in the $\Delta$-region $[49,92]$, is not feasible for $\mathrm{K} \wedge$ production, due to the lack of knowledge about the appropriate phase shifts.

\section{A.2 Effective Lagrangians for $p(\gamma, K) Y$ Reactions}

Sec. A.1 summarizes the interaction Lagrangians and the propagators which are relevant for the $\mathrm{K}^{+} \wedge$ photoproduction case. However, open-strangeness photoproduction on the nucleon involves six different isospin channels in total:

$$
\begin{aligned}
& \gamma+\mathrm{p} \rightarrow \mathrm{K}^{+}+\Lambda^{0}, \quad \gamma+\mathrm{n} \rightarrow \mathrm{K}^{0}+\Lambda^{0}, \\
& \gamma+\mathrm{p} \rightarrow \mathrm{K}^{+}+\Sigma^{0}, \quad \gamma+\mathrm{n} \rightarrow \mathrm{K}^{0}+\Sigma^{0}, \\
& \gamma+\mathrm{p} \rightarrow \mathrm{K}^{0}+\Sigma^{+}, \quad \gamma+\mathrm{n} \rightarrow \mathrm{K}^{+}+\Sigma^{-} .
\end{aligned}
$$

In what follows, we will first summarize the extensions to Sec. A.1 which turn out to be necessary to cover all of the above six channels. Further on, we will point out relations amongst the coupling constants over the different isospin channels.

\section{A.2.1 Born Terms}

The electromagnetic interaction Lagrangians in Sec. A.1 were specifically designed for the $\mathrm{p}\left(\gamma, \mathrm{K}^{+}\right) \wedge$ process. For a more general $\mathrm{N}(\gamma, \mathrm{K}) \mathrm{Y}$ reaction, global electromagnetic interactions can be constructed.

The Lagrangian describing the general coupling of a photon to a kaon is:

$$
\mathcal{L}_{\gamma K K}=-i e_{K}\left(K^{\dagger} \partial_{\mu} K-K \partial_{\mu} K^{\dagger}\right) A^{\mu}
$$




\begin{tabular}{||lr||}
\hline \hline $\begin{array}{l}\text { Anomalous } \\
\text { magnetic moment }\end{array}$ & Value \\
\hline$\kappa_{p}$ & 1.793 \\
$\kappa_{n}$ & -1.913 \\
$\kappa_{\Lambda}$ & -0.613 \\
$\kappa_{\Sigma^{+}}$ & 1.458 \\
$\kappa_{\Sigma^{-}}$ & -0.160 \\
$\kappa_{\Sigma^{0}}$ & 0.790 \\
$\left|\kappa_{\Sigma^{0}}\right|$ & 1.610 \\
\hline \hline
\end{tabular}

Table A.1 The anomalous magnetic moments used in the calculations presented in this work. The numeric values are from Ref. [58].

with $e_{\mathrm{K}}$ the charge of the kaon. It is obvious that this interaction vanishes for neutral kaon production.

For every intermediate baryon in the Born diagrams, there exists a general electromagnetic interaction of the type:

$$
-e_{\mathrm{B}} \overline{\mathrm{B}} \gamma_{\mu} \mathrm{B} A^{\mu}+\frac{e \kappa_{\mathrm{B}}}{4 m_{p}} \overline{\mathrm{B}} \sigma_{\mu \nu} \mathrm{BF}{ }^{\mu \nu}
$$

The first term corresponds to the coupling of the real photon to the charge of the particle and is proportional to the charge $e_{\mathrm{B}}$. Consequently, this term vanishes if the (real) photon couples to a neutral baryon like the neutron, $\Lambda$ or $\Sigma^{0}$. The anomalous magnetic moments $\kappa_{B}$, which determine the strength of the spin coupling part $\sigma^{\mu \nu}$, are summarized in Table A.1. The $\Sigma^{0}$ hyperon has a very short lifetime $\left(7.4 \cdot 10^{-20} \mathrm{~s}\right)$ and decays electromagnetically via $\Sigma^{0} \rightarrow \Lambda+\gamma$. This decay is too fast to determine its magnetic moment experimentally. Therefore, we resort to a quark model prediction for this quantity [93]. Remark that the Particle Data Group tables [58] collects the magnetic moments $\mu_{\mathrm{B}}$. The relation with the anomalous magnetic moments $\kappa_{\mathrm{B}}$ given by:

$$
\mu_{\mathrm{B}}=\kappa_{\mathrm{B}}+\frac{e_{\mathrm{B}}}{e} .
$$

Note also that all values of $\mu_{\mathrm{B}}$ in Ref. [58] are normalized to the nuclear magneton $\left(\mu_{N}=e / 2 m_{p}\right)$, which is in agreement with our definition of the interaction Lagrangians in Sec. A.1. 
A point of confusion is the sign of $\kappa_{\Sigma^{0}} \wedge$, since this is experimentally not accessible. Using the conventions of de Swart [47], based on (ideal) SU(3) symmetry and commonly used for the hadronic vertices, it turns out that the product $\kappa_{\Sigma^{0}} \wedge \cdot g_{K^{+}} \Sigma^{0} p$ must have the opposite sign of the coupling constant $g_{K^{+}} \wedge_{p}$. In the same spirit, $g_{K^{+}} \wedge p$ is predicted to be negative and $g_{K^{+} \Sigma^{0} p}$ positive. Consequently, we take:

$$
\kappa_{\Sigma 0} \wedge=+1.61
$$

as value for the electromagnetic $\Sigma^{0} \Lambda$ transition moment.

\section{A.2.2 Isospin Symmetry and Coupling Constants}

Isospin symmetry considerations are extremely useful tools to establish ranges and relative signs between series of coupling constants. In this section we sketch how isospin arguments can be used to construct relations between the different hadronic and electromagnetic coupling constants which are required to describe photoinduced open strangeness production on the nucleon. We assume the isospin symmetry of the various meson and baryon multiplets to be exact. In what follows we will briefly address both hadronic and electromagnetic coupling constants.

\section{Hadronic Decays of $\mathrm{N}^{*}$ and $\Delta^{*}$ Resonances}

The calculation of hadronic transitions of baryon resonances poses a challenging task to Constituent Quark Models (CQM). The major difficulty of such models is to determine the structure of the operators which govern the decay mechanism. This reflects the insufficient basic insight into the quark dynamics in low energy hadron phenomenology. Most CQM's that study hadronic decays of baryon resonances (for a recent example see Ref. [94]), start from a transition operator at quark level which does not contain isospin-dependent terms. In such a model, the amplitude for a pseudo-scalar hadronic decay of a non-strange baryon into a KY-system of the type:

$$
\mathrm{B}\left(\mathrm{I}_{1}, \mathrm{M}_{1}\right) \longrightarrow \mathrm{K}\left(\mathrm{I}_{2}, \mathrm{M}_{2}\right)+\mathrm{Y}\left(\mathrm{I}_{3}, \mathrm{M}_{3}\right)
$$

is proportional to the isospin part:

$$
\frac{(-1)^{\mathrm{I}_{2}-\mathrm{I}_{1}}}{\sqrt{2 \mathrm{I}_{1}+1}}\left\langle\mathrm{I}_{2} M_{2} \mathrm{I}_{3} M_{3} \mid \mathrm{I}_{1} M_{1}\right\rangle\left\langle\mathrm{I}_{2}\left\|\widehat{T}^{\left(\mathrm{I}_{3}\right)}\right\| \mathrm{I}_{1}\right\rangle,
$$


where $I_{i}$ and $M_{i}$ are the isospin and isospin projection of the respective particles and $\widehat{T}^{(I)}$ denotes a spherical tensor operator of rank I. From the expression (A.30), one easily obtains the following relations between the different isospin channels in $\mathrm{N} \rightarrow \mathrm{K} \Sigma:$

$$
g_{K^{+} \Sigma^{0} p}=\frac{g_{K^{0} \Sigma^{+} p}}{\sqrt{2}}=-g_{K^{0} \Sigma^{0} n}=\frac{g_{K^{+} \Sigma^{-} n}}{\sqrt{2}} .
$$

In determining these relations we adopted the following conventions for the isospin states of the physical $\Sigma$ particles:

$$
\begin{array}{ll}
\Sigma^{+}: & -|\mathrm{I}=1, M=+1\rangle, \\
\Sigma^{0}: & +|\mathrm{I}=1, M=0\rangle, \\
\Sigma^{-}: & +|\mathrm{I}=1, M=-1\rangle .
\end{array}
$$

For the hadronic decays of the type $\mathrm{N} \rightarrow \mathrm{K} \Lambda$, starting from Eq. (A.30), even simpler relations can be written down:

$$
\mathrm{g}_{\wedge \mathrm{K}^{+} \mathrm{p}}=\mathrm{g}_{\wedge \mathrm{K}^{0} \mathrm{n}} .
$$

We now consider hadronic decays of the type $\Delta \rightarrow \mathrm{K} \Sigma$. Defining the corresponding isospin states for the $\Delta^{+, 0}$ particles as $\left|I=\frac{3}{2}, M= \pm \frac{1}{2}\right\rangle$, one obtains the following relations from Eq. (A.30):

$$
\mathrm{g}_{\mathrm{K}^{+} \Sigma^{0} \Delta^{+}}=-\sqrt{2} \mathrm{~g}_{\mathrm{K}^{0} \Sigma^{+} \Delta^{+}}=\mathrm{g}_{\mathrm{K}^{0} \Sigma^{0} \Delta^{0}}=\sqrt{2} \mathrm{~g}_{\mathrm{K}^{+} \Sigma^{-} \Delta^{0}} \text {. }
$$

Note that all relations contained in Eqs. (A.31), (A.33) and (A.34), also hold when a $\mathrm{N}^{*}, \mathrm{~K}^{*}, \Sigma^{*}$ or $\Lambda^{*}$ resonance is involved at the vertex.

\section{Electromagnetic vertices}

For the determination of some of the electromagnetic coupling constants, one can rely on experimental quantities. The measured decay widths for the $\mathrm{K}^{*+}(892)$ and $\mathrm{K}^{* 0}(892)$ vector mesons are [58]:

$$
\begin{aligned}
\Gamma_{\mathrm{K}^{*+} \rightarrow \mathrm{K}^{+} \gamma} & =50 \pm 5 \mathrm{keV}, \\
\Gamma_{\mathrm{K}^{* 0} \rightarrow \mathrm{K}^{0} \gamma} & =116 \pm 10 \mathrm{keV} .
\end{aligned}
$$

In principle, one can determine the value of the magnetic transition moment on the basis of the proportionality $\mathrm{K}_{\mathrm{K}^{*} \mathrm{~K}}^{2} \sim \Gamma_{\mathrm{K}^{*} \rightarrow \mathrm{K} \gamma}$. Within the context of isobar models, 
however, the coupling constants are frequently considered as "effective couplings" wherein, for example, part of final-state interaction effects are absorbed. It is a common procedure to use only the ratio of the measured decay widths to connect isospin related coupling constants. This leads to the following expression:

$$
\frac{\mathrm{K}_{\mathrm{K}^{* 0}}^{2} \mathrm{~K}^{0}}{\mathrm{~K}_{\mathrm{K}^{*+}}^{2} \mathrm{~K}^{+}}=\frac{\Gamma_{\mathrm{K}^{* 0} \rightarrow \mathrm{K}^{0} \gamma}}{\Gamma_{\mathrm{K}^{*+} \rightarrow \mathrm{K}^{+} \gamma}},
$$

or:

$$
\mathrm{K}_{\mathrm{K}^{* 0}} \mathrm{~K}^{0}=-1.52 \mathrm{~K}_{\mathrm{K}^{*+}} \mathrm{K}^{+} .
$$

The relative sign in the last expression was allocated on the basis of a CQM prediction [95].

The nucleon magnetic transition moments are related to the photohelicity amplitudes through the interaction Lagrangians. From the isospin structure of the $\mathrm{N}^{*}$ helicity amplitudes, it is easily proven that they are sensitive to the isospin of the final state. To determine the electromagnetic vertex coupling at a neutron target from the knowledge of the electromagnetic coupling at a proton target, those differences have to be taken into account. We adopt the same procedure as for the vector meson transition moments and use the experimental amplitudes as a conversion coefficient. The expressions, which directly follow from the interaction Lagrangians, read:

$$
\begin{array}{rlrl}
\operatorname{spin}-\frac{1}{2}: & \frac{\kappa_{N^{*} n}}{\kappa_{N^{*} p}} & =\frac{A_{1 / 2}^{n}}{A_{1 / 2}^{p}}, \\
\operatorname{spin}-\frac{3}{2}: & \frac{\kappa_{N^{*} n}^{(1)}}{\kappa_{N^{*} p}^{(1)}} & =\frac{\sqrt{3} A_{1 / 2}^{n} \pm A_{3 / 2}^{n}}{\sqrt{3} A_{1 / 2}^{p} \pm A_{3 / 2}^{p}}, \\
\frac{\kappa_{N^{*} n}^{(2)}}{\kappa_{N^{*} p}^{(2)}} & =\frac{\sqrt{3} A_{1 / 2}^{n}-\frac{m_{p}}{m_{N^{*}}} A_{3 / 2}^{n}}{\sqrt{3} A_{1 / 2}^{p}-\frac{m_{p}}{m_{N^{*}}} A_{3 / 2}^{p}},
\end{array}
$$

where +/- refers to even/odd parity. Note that some of these helicity amplitudes are rather poorly known, especially those of the neutron. For the electromagnetic decay of the $\Delta^{*}$ resonances, the following simple relation holds:

$$
\mathrm{\kappa}_{\Delta *} \mathrm{p}=\mathrm{\kappa}_{\Delta *} \mathrm{n}
$$

regardless of the spin state of the $\Delta^{*}$ resonance. This is not the case for the electromagnetic decay of $\Sigma^{*}$ resonances. In principle, one can make use of the same 
procedure adopted for the $\mathrm{K}^{*}$ and $\mathrm{N}^{*}$ transition moments and take the ratio of the helicity amplitudes as a conversion coefficient. Due to the lack of knowledge about the latter quantities, we have used ratios of the $\Sigma$ ground state transition moments as conversion coefficients. This produces the following relations:

$$
\kappa_{\Sigma^{*} \Sigma^{0}}=\frac{\mu_{\Sigma^{0}}}{\mu_{\Sigma^{+}}} \kappa_{\Sigma^{*} \Sigma^{+}}=\frac{\mu_{\Sigma^{0}}}{\mu_{\Sigma^{-}}} \kappa_{\Sigma^{*} \Sigma^{-}}
$$

in which we have used $\mu_{\Sigma^{+}}, \mu_{\Sigma^{-}}$, and $\mu_{\Sigma^{0}}$ values as deduced from the $\kappa^{\prime} s$ in Table. A.1.

\section{A.2.3 Numerical Values}

In Figs. 3.5 and 3.22, the extracted resonance coupling constants are plotted for the three different models which are used in the discussions of the $K^{+} \Lambda$ and $K \Sigma$ photoproduction processes. As a matter of fact, the model calculations depend on more parameters than the resonance coupling constants which are presented in those figures. For the sake of completeness, all numbers entering the isobar model calculations for the $\mathrm{K}^{+} \Lambda$ and $\mathrm{K}^{+} \Sigma^{0}$ photoproduction reaction are collected in Tables A.2 and A.3. The normalization conventions with regard to those coupling constants and their connection with the structure of the interaction Lagrangians have been summarized in App. A.1.1. A list of relevant particles in the strangeness production processes and some of their properties can be found in Table A.4.

\section{A.3 Electromagnetic Form Factors for $p\left(e, e^{\prime} K\right) Y$ Reactions}

This subsection summarizes the adopted function forms for the electromagnetic form factors in the $p\left(e, e^{\prime} \mathrm{K}\right) \mathrm{Y}$ calculations. Those form factors reflect the composite nature of the hadrons when they are probed by virtual photons. We first discuss the baryonic form factors and then proceed with the mesonic ones.

\section{A.3.1 Baryonic Form Factors}

The $\gamma p p$ vertex is extensively studied in the literature and different parameterizations for the form factors are suggested. For the Dirac $\left(F_{1}\right)$ and Pauli $\left(F_{2}\right)$ form factors, we have used a parameterization from Gari and Krümpelmann [96-98] which was recently modified by Lomon [99]. This model combines the low $\mathrm{Q}^{2}$ vector 


\begin{tabular}{|c|c|c|c|c|}
\hline & & model A & model B & model C \\
\hline \multirow[t]{2}{*}{ Born terms } & $g_{K^{+}{ }_{\wedge p}} / \sqrt{4 \pi}$ & -3.191 & -2.908 & $-4.044 \cdot 10^{-1}$ \\
\hline & $g_{K}+\Sigma p / \sqrt{4 \pi}$ & 1.007 & $9.799 \cdot 10^{-1}$ & $3.131 \cdot 10^{-1}$ \\
\hline \multirow[t]{2}{*}{$\mathrm{K}^{*}$} & $\mathrm{G}_{\mathrm{K}^{*}}^{v}$ & $-2.216 \cdot 10^{-2}$ & $8.792 \cdot 10^{-2}$ & $1.325 \cdot 10^{-1}$ \\
\hline & $\mathrm{G}_{\mathrm{K}^{*}}^{\mathrm{t}}$ & $-2.563 \cdot 10^{-1}$ & $-1.580 \cdot 10^{-1}$ & $-1.671 \cdot 10^{-2}$ \\
\hline \multirow[t]{2}{*}{$\mathrm{K}_{1}$} & $\mathrm{G}_{\mathrm{K}_{1}}^{v}$ & $-3.221 \cdot 10^{-1}$ & $-6.547 \cdot 10^{-2}$ & $7.992 \cdot 10^{-2}$ \\
\hline & $\mathrm{G}_{\mathrm{K}_{1}}^{\mathrm{t}}$ & $-2.335 \cdot 10^{-1}$ & $-9.509 \cdot 10^{-1}$ & $-1.083 \cdot 10^{-1}$ \\
\hline$S_{01}(1800)$ & $\mathrm{G}_{\mathrm{S}_{01}}$ & & -4.389 & \\
\hline$P_{01}(1810)$ & $\mathrm{G}_{P_{01}}$ & & -1.750 & \\
\hline$S_{11}(1650)$ & $\mathrm{G}_{\mathrm{S}_{11}}$ & $-8.418 \cdot 10^{-2}$ & $-4.227 \cdot 10^{-2}$ & $-8.364 \cdot 10^{-2}$ \\
\hline$P_{11}(1710)$ & $\mathrm{G}_{P_{11}}$ & $-3.706 \cdot 10^{-2}$ & $-9.538 \cdot 10^{-3}$ & $-7.307 \cdot 10^{-2}$ \\
\hline \multirow[t]{5}{*}{$P_{13}(1720)$} & $\mathrm{G}_{\mathrm{P}_{13}}^{(1)}$ & $4.165 \cdot 10^{-5}$ & $1.719 \cdot 10^{-2}$ & $1.748 \cdot 10^{-3}$ \\
\hline & $\mathrm{G}_{\mathrm{P}_{13}}^{(2)}$ & $5.593 \cdot 10^{-3}$ & $1.502 \cdot 10^{-2}$ & $6.820 \cdot 10^{-2}$ \\
\hline & $\mathrm{X}_{\mathrm{P}_{13}}$ & 9.055 & 1.240 & $-2.034 \cdot 10^{-1}$ \\
\hline & $\mathrm{Y}_{\mathrm{P}_{13}}$ & 9.601 & -1.552 & -25.951 \\
\hline & $Z_{P_{13}}$ & -1.154 & $-7.810 \cdot 10^{-2}$ & $-3.558 \cdot 10^{-1}$ \\
\hline \multirow[t]{5}{*}{$\mathrm{D}_{13}(1895)$} & $G_{D_{13}}^{(1)}$ & $-2.919 \cdot 10^{-2}$ & $-3.556 \cdot 10^{-2}$ & $-6.261 \cdot 10^{-2}$ \\
\hline & $G_{D_{13}}^{(2)^{3}}$ & $-1.322 \cdot 10^{-1}$ & $-1.096 \cdot 10^{-1}$ & $-1.132 \cdot 10^{-1}$ \\
\hline & $X_{D_{13}}^{D_{13}}$ & 1.843 & -6.333 & -3.666 \\
\hline & $Y_{D_{13}}$ & $2.525 \cdot 10^{-1}$ & 1.142 & -1.585 \\
\hline & $Z_{D_{13}}$ & $-5.262 \cdot 10^{-2}$ & $-5.964 \cdot 10^{-2}$ & $-1.851 \cdot 10^{-1}$ \\
\hline \multirow[t]{2}{*}{ cutoff mass } & $\Lambda_{\text {born }}$ & 412.92 & 1538.17 & 1855.95 \\
\hline & $\Lambda_{\text {res }}$ & 1524.76 & 2039.73 & 1601.41 \\
\hline
\end{tabular}

Table A.2 Numerical values of the coupling constants and hadronic cutoff masses (in MeV) in the isobar model calculations for the $p\left(\gamma, \mathrm{K}^{+}\right) \wedge$ process. 


\begin{tabular}{|c|c|c|c|c|}
\hline & & model A & model B & model C \\
\hline \multirow[t]{2}{*}{ Born terms } & $g_{K}+\Sigma p / \sqrt{4 \pi}$ & $9.949 \cdot 10^{-1}$ & $9.215 \cdot 10^{-1}$ & $2.811 \cdot 10^{-1}$ \\
\hline & $g_{K^{+} \wedge p} / \sqrt{4 \pi}$ & -4.487 & -3.017 & $-2.261 \cdot 10^{-1}$ \\
\hline \multirow[t]{2}{*}{$\mathrm{K}^{*}$} & $\mathrm{G}_{\mathrm{K}^{*}}^{v}$ & $6.926 \cdot 10^{-2}$ & $7.598 \cdot 10^{-2}$ & $6.828 \cdot 10^{-2}$ \\
\hline & $\mathrm{G}_{\mathrm{K}^{*}}^{\mathrm{t}}$ & $8.047 \cdot 10^{-2}$ & $4.087 \cdot 10^{-2}$ & $1.411 \cdot 10^{-1}$ \\
\hline$P_{01}(1810)$ & $\mathrm{G}_{\mathrm{P}_{01}}$ & & 21.838 & \\
\hline$P_{11}(1880)$ & $\mathrm{G}_{\mathrm{P}_{11}}$ & & -9.451 & \\
\hline$S_{11}(1650)$ & $\mathrm{G}_{\mathrm{S}_{11}}$ & $-4.568 \cdot 10^{-2}$ & $-4.516 \cdot 10^{-3}$ & $-2.511 \cdot 10^{-2}$ \\
\hline$P_{11}(1710)$ & $\mathrm{G}_{P_{11}}$ & $-1.213 \cdot 10^{-1}$ & $-1.583 \cdot 10^{-1}$ & $-1.879 \cdot 10^{-1}$ \\
\hline \multirow[t]{5}{*}{$P_{13}(1720)$} & $G_{P_{13}}^{(1)}$ & $2.367 \cdot 10^{-2}$ & $1.706 \cdot 10^{-2}$ & $2.699 \cdot 10^{-2}$ \\
\hline & $\mathrm{G}_{\mathrm{P}_{13}}^{(2)}$ & $5.238 \cdot 10^{-2}$ & $8.343 \cdot 10^{-2}$ & $5.213 \cdot 10^{-2}$ \\
\hline & $X_{P_{13}}$ & 12.351 & 6.943 & 14.863 \\
\hline & $Y_{P_{13}}$ & 3.781 & 4.765 & 3.861 \\
\hline & $Z_{P_{13}}$ & -1.122 & -1.129 & -1.089 \\
\hline$S_{31}(1900)$ & $\mathrm{G}_{S_{31}}$ & $5.131 \cdot 10^{-2}$ & $4.279 \cdot 10^{-2}$ & $4.351 \cdot 10^{-2}$ \\
\hline$P_{31}(1910)$ & $\mathrm{G}_{P_{31}}$ & $3.726 \cdot 10^{-1}$ & $3.599 \cdot 10^{-1}$ & $3.920 \cdot 10^{-1}$ \\
\hline \multirow[t]{2}{*}{ cutoff mass } & $\Lambda_{\text {born }}$ & 439.68 & 1605.04 & 2509.22 \\
\hline & $\Lambda_{\text {res }}$ & 1616.20 & 1602.43 & 1601.54 \\
\hline
\end{tabular}

Table A.3 Numerical values of the coupling constants and hadronic cutoff masses (in $\mathrm{MeV}$ ) in the isobar model calculations for the $p\left(\gamma, \mathrm{K}^{+}\right) \Sigma^{0}$ process. Note that those numbers can also be applied to the $p\left(\gamma, \mathrm{K}^{0}\right) \Sigma^{+}$process with the aid the isospin conversion relations summarized in App. A.2.2. 


\begin{tabular}{|c|c|c|c|c|c|}
\hline Notation & $\mathrm{L}_{(2 \mathrm{I}),(2 \mathrm{~J})}$ & $\mathrm{J}^{\pi}$ & Mass (MeV) & Width (MeV) & PDG-ranking \\
\hline$p$ & $P_{11}$ & $1 / 2^{+}$ & 938.272 & & \\
\hline$n$ & $P_{11}$ & $1 / 2^{+}$ & 939.565 & & \\
\hline$\Lambda$ & $P_{01}$ & $1 / 2^{+}$ & 1115.68 & & \\
\hline$\Sigma^{0}$ & $P_{11}$ & $1 / 2^{+}$ & 1192.64 & & \\
\hline$\Sigma^{+}$ & $P_{11}$ & $1 / 2^{+}$ & 1189.37 & & \\
\hline$\Sigma^{-}$ & $P_{11}$ & $1 / 2^{+}$ & 1197.45 & & \\
\hline $\mathrm{K}^{+}$ & & $0^{-}$ & 493.677 & & \\
\hline $\mathrm{K}^{0}$ & & $0^{-}$ & 497.672 & & \\
\hline $\mathrm{K}^{*}(892)^{+}$ & & $1^{-}$ & 891.66 & 50.8 & \\
\hline$K^{*}(892)^{\rho}$ & & $1^{-}$ & 896.10 & 50.7 & \\
\hline$K_{1}(1270)$ & & 1 & 1273.0 & 90.0 & \\
\hline$N(1440)$ & $P_{1}$ & $1 / 2^{+}$ & 1440 & 350 & $* * * *$ \\
\hline$N(1520)$ & $\mathrm{Q}_{3}$ & $3 / 2$ & 1520 & 120 & $* * * *$ \\
\hline$N(1535)$ & $\$_{1}$ & $1 / 2$ & 1535 & 150 & $* * * *$ \\
\hline$N(1650)$ & $\$_{1}$ & $1 / 2$ & 1650 & 150 & $* * * *$ \\
\hline$N(1675)$ & $\mathrm{Q}_{5}$ & $5 / 2$ & 1670 & 150 & $* * * *$ \\
\hline$N(1680)$ & $F_{5}$ & $5 / 2^{+}$ & 1680 & 130 & $* * * *$ \\
\hline $\mathrm{N}(1700)$ & $\mathrm{Q}_{3}$ & $3 / 2$ & 1700 & 100 & $* * *$ \\
\hline$N(1710)$ & $P_{1}$ & $1 / 2^{+}$ & 1710 & 100 & $* * *$ \\
\hline$N(1720)$ & $P_{3}$ & $3 / 2^{+}$ & 1720 & 150 & $* * * *$ \\
\hline$N(1895)$ & $\mathrm{Q}_{3}$ & $3 / 2$ & 1895 & 350 & $\dagger$ \\
\hline$N(1895)$ & $P_{3}$ & $3 / 2^{+}$ & 1895 & 350 & $\dagger$ \\
\hline$N(1900)$ & $P_{3}$ & $3 / 2^{+}$ & 1900 & 500 & ** \\
\hline$\wedge(1405)$ & $\S_{1}$ & $1 / 2^{-}$ & 1406 & 50 & $* * * *$ \\
\hline$\wedge(1520)$ & $D_{3}$ & $3 / 2$ & 1520 & 15.6 & $* * * *$ \\
\hline$\Lambda(1600)$ & $B_{1}$ & $1 / 2^{+}$ & 1600 & 150 & $* * *$ \\
\hline$\Lambda(1670)$ & $\$_{1}$ & $1 / 2$ & 1670 & 35 & $* * * *$ \\
\hline$\wedge(1690)$ & $D_{3}$ & $3 / 2$ & 1690 & 60 & $* * * *$ \\
\hline$\wedge(1800)$ & $\oint_{1}$ & $1 / 2$ & 1800 & 300 & $* * *$ \\
\hline$\wedge(1810)$ & $B_{1}$ & $1 / 2^{+}$ & 1810 & 150 & $* * *$ \\
\hline$\wedge(1820)$ & 55 & $5 / 2^{+}$ & 1820 & 80 & $* * * *$ \\
\hline$\wedge(1830)$ & 85 & $5 / 2$ & 1830 & 95 & $* * * *$ \\
\hline$\Lambda(1890)$ & $B_{3}$ & $3 / 2^{+}$ & 1890 & 100 & $* * * *$ \\
\hline$\Sigma(1385)$ & $P_{3}$ & $3 / 2^{+}$ & 1385 & 36 & $* * * *$ \\
\hline$\Sigma(1660)$ & $P_{1}$ & $1 / 2^{+}$ & 1660 & 100 & $* * *$ \\
\hline$\Sigma(1670)$ & $\mathrm{Q}_{3}$ & $3 / 2$ & 1670 & 60 & $* * * *$ \\
\hline$\Sigma(1750)$ & $\$_{1}$ & $1 / 2$ & 1750 & 90 & $* * *$ \\
\hline$\Sigma(1775)$ & $\mathrm{Q}_{5}$ & $5 / 2$ & 1775 & 120 & $* * * *$ \\
\hline$\Sigma(1880)$ & $P_{1}$ & $1 / 2^{+}$ & 1880 & 200 & $* *$ \\
\hline$\Delta(1232)$ & $B_{3}$ & $3 / 2^{+}$ & 1232 & 120 & $* * * *$ \\
\hline$\Delta(1600)$ & $B_{3}$ & $3 / 2^{+}$ & 1600 & 350 & $* * *$ \\
\hline$\Delta(1620)$ & $\S_{1}$ & $1 / 2$ & 1620 & 150 & $* * * *$ \\
\hline$\Delta(1700)$ & 马3 & $3 / 2$ & 1700 & 300 & $* * * *$ \\
\hline$\Delta(1900)$ & $\S_{1}$ & $1 / 2$ & 1900 & 200 & ** \\
\hline$\Delta(1905)$ & 55 & $5 / 2^{+}$ & 1905 & 350 & $* * * *$ \\
\hline$\Delta(1910)$ & $B_{1}$ & $1 / 2^{+}$ & 1910 & 250 & $* * * *$ \\
\hline$\Delta(1920)$ & $B_{3}$ & $3 / 2^{+}$ & 1920 & 200 & **** \\
\hline
\end{tabular}

Table A.4 Properties of known baryons which can play a role in the reaction dynamics of strangeness production processes. The nucleon resonances denoted with a $\dagger$ are "missing resonances". Note that for the hyperon resonances, the quantum numbers are denoted as $\mathrm{L}_{(\mathrm{I}),(2 \mathrm{~J})}$. The values are from the Particle Data Group [58]. 


\begin{tabular}{lcccccccc}
\hline \hline$\kappa_{i v}$ & $\kappa_{i s}$ & $g_{\rho} / f_{\rho}$ & $\kappa_{\rho}$ & $g_{\omega} / f_{\omega}$ & $\kappa_{\omega}$ & $g_{\phi} / f_{\phi}$ & $\kappa_{\phi}$ & $\mu_{\phi}$ \\
\hline 3.706 & -0.12 & 0.4466 & 4.3472 & 0.4713 & 21.762 & -0.8461 & 11.849 & 1.1498 \\
\hline \hline$\Lambda_{1}^{\rho, \omega, \phi}$ & $\Lambda_{1}^{\mathrm{D}}$ & $\Lambda_{2}$ & $\Lambda_{\mathrm{QCD}}$ & & & & & \\
\hline 0.9006 & 1.7038 & 1.1336 & 0.0312 & & & & & \\
\hline \hline
\end{tabular}

Table A.5 Parameters for the nucleon electromagnetic form factors obtained by Lomon [99] (model GR(3)). The $\Lambda$ 's are given in GeV.

meson dominance hypothesis with the high $\mathrm{Q}^{2}$ perturbative QCD approach. The form factors are expressed in terms of their isoscalar (is) and isovector (iv) parts, according to:

$$
\begin{aligned}
& \mathrm{F}_{1}^{\mathrm{p}} \equiv \frac{1}{2}\left(\mathrm{~F}_{1}^{\mathrm{is}}+\mathrm{F}_{1}^{\mathrm{iv}}\right) \\
& \mathrm{F}_{2}^{\mathrm{p}} \equiv \frac{1}{2 \kappa_{\mathrm{p}}}\left(\kappa_{i s} \mathrm{~F}_{2}^{\mathrm{is}}+\kappa_{i v} \mathrm{~F}_{2}^{\mathrm{i} v}\right) .
\end{aligned}
$$

The isoscalar and isovector parts are defined as:

$$
\begin{aligned}
\mathrm{F}_{1}^{i \nu}\left(Q^{2}\right)= & \frac{g_{\rho}}{f_{\rho}} \frac{m_{\rho}^{2}}{m_{\rho}^{2}+Q^{2}} F_{1}^{\rho}\left(Q^{2}\right)+\left(1-\frac{g_{\rho}}{f_{\rho}}\right) F_{1}^{D}\left(Q^{2}\right) \\
\kappa_{i v} F_{2}^{i v}\left(Q^{2}\right)= & \kappa_{\rho} \frac{g_{\rho}}{f_{\rho}} \frac{m_{\rho}^{2}}{m_{\rho}^{2}+Q^{2}} F_{2}^{\rho}\left(Q^{2}\right)+\left(\kappa_{i v}-\kappa_{\rho} \frac{g_{\rho}}{f_{\rho}}\right) F_{2}^{D}\left(Q^{2}\right) \\
F_{1}^{i s}\left(Q^{2}\right)= & \frac{g_{\omega}}{f_{\omega}} \frac{m_{\omega}^{2}}{m_{\omega}^{2}+Q^{2}} F_{1}^{\omega}\left(Q^{2}\right)+\frac{g_{\phi}}{f_{\phi}} \frac{m_{\phi}^{2}}{m_{\phi}^{2}+Q^{2}} F_{1}^{\phi}\left(Q^{2}\right) \\
& +\left(1-\frac{g_{\omega}}{f_{\omega}}\right) F_{1}^{D}\left(Q^{2}\right), \\
\kappa_{i s} F_{2}^{i s}\left(Q^{2}\right)= & \kappa_{\omega} \frac{g_{\omega}}{f_{\omega}} \frac{m_{\omega}^{2}}{m_{\omega}^{2}+Q^{2}} F_{2}^{\omega}\left(Q^{2}\right)+\kappa_{\phi} \frac{g_{\phi}}{f_{\phi}} \frac{m_{\phi}^{2}}{m_{\phi}^{2}+Q^{2}} F_{2}^{\phi}\left(Q^{2}\right) \\
& +\left(\kappa_{i s}-\kappa_{\omega} \frac{g_{\omega}}{f_{\omega}}-\kappa_{\phi} \frac{g_{\phi}}{f_{\phi}}\right) F_{2}^{D}\left(Q^{2}\right) \cdot
\end{aligned}
$$


Herein, $g_{\rho}, g_{\omega}$ and $g_{\phi}$ are the vector meson nucleon coupling constants. Further, the parameters $m_{\rho}^{2} / f_{\rho}, m_{\omega}^{2} / f_{\omega}$ and $m_{\phi}^{2} / f_{\phi}$ determine the vector meson photon coupling and the $\kappa^{\prime} s$ are the magnetic moments of the corresponding particles. $F_{i}^{\rho}, F_{i}^{\omega}$ and $F_{i}^{\phi}$ denote the meson nucleon form factors. $F_{i}^{D}$ describes the nucleon non-resonant quark structure, which is responsible for the asymptotic behavior at $\mathrm{Q}^{2} \rightarrow \infty$. To achieve a smooth transition between the low and high $\mathrm{Q}^{2}$ domains, the following parameterization is adopted for these meson nucleon form factors:

$$
\begin{aligned}
& \mathrm{F}_{1}^{\alpha}\left(\mathrm{Q}^{2}\right)=\frac{\Lambda_{1}^{2}}{\Lambda_{1}^{2}+\widetilde{\mathrm{Q}}^{2}} \frac{\Lambda_{2}^{2}}{\Lambda_{2}^{2}+\widetilde{\mathrm{Q}}^{2}}, \\
& \mathrm{~F}_{2}^{\alpha}\left(\mathrm{Q}^{2}\right)=\left[\frac{\Lambda_{1}^{2}}{\Lambda_{1}^{2}+\widetilde{\mathrm{Q}}^{2}}\right]^{2} \frac{\Lambda_{2}^{2}}{\Lambda_{2}^{2}+\widetilde{\mathrm{Q}}^{2}},
\end{aligned}
$$

for $\alpha=\rho, \omega, D$. For the $\phi$ form factors, an extra factor is added:

$$
\begin{aligned}
& \mathrm{F}_{1}^{\phi}\left(\mathrm{Q}^{2}\right)=\frac{\Lambda_{1}^{2}}{\Lambda_{1}^{2}+\widetilde{\mathrm{Q}}^{2}} \frac{\Lambda_{2}^{2}}{\Lambda_{2}^{2}+\widetilde{\mathrm{Q}}^{2}}\left[\frac{\mathrm{Q}^{2}}{\Lambda_{1}^{2}+\mathrm{Q}^{2}}\right]^{1.5}, \\
& \mathrm{~F}_{2}^{\phi}\left(\mathrm{Q}^{2}\right)=\left[\frac{\Lambda_{1}^{2}}{\Lambda_{1}^{2}+\widetilde{\mathrm{Q}}^{2}}\right]^{2} \frac{\Lambda_{2}^{2}}{\Lambda_{2}^{2}+\widetilde{\mathrm{Q}}^{2}}\left[\frac{\Lambda_{1}^{2}}{\mu_{\phi}^{2}} \frac{\mu_{\phi}^{2}+\mathrm{Q}^{2}}{\Lambda_{1}^{2}+\mathrm{Q}^{2}}\right]^{1.5} .
\end{aligned}
$$

In order to approach the logarithmic $\mathrm{Q}^{2}$ behavior predicted by $\mathrm{pQCD}, \widetilde{\mathrm{Q}}^{2}$ is defined as:

$$
\widetilde{\mathrm{Q}}^{2}=\mathrm{Q}^{2} \ln \left(\frac{\Lambda_{2}^{2}+\mathrm{Q}^{2}}{\Lambda_{\mathrm{QCD}}^{2}}\right) / \ln \left(\frac{\Lambda_{2}^{2}}{\Lambda_{\mathrm{QCD}}^{2}}\right) .
$$

The values of the parameters, as reexamined by Lomon [99] (model GK(3) in the reference), are given in Table A.5.

At the $\gamma \wedge Y$ vertices (with $Y=\Lambda, \Sigma^{0}$ ), we have used the neutron form factors, defined as:

$$
\begin{aligned}
& \mathrm{F}_{1}^{\wedge, \Sigma^{0}}=\mathrm{F}_{1}^{\mathrm{n}} \equiv \frac{1}{2}\left(\mathrm{~F}_{1}^{\mathrm{is}}-\mathrm{F}_{1}^{i \nu}\right), \\
& \mathrm{F}_{2}^{\wedge, \Sigma^{0}}=\mathrm{F}_{2}^{n} \equiv \frac{1}{2 \kappa_{\mathrm{n}}}\left(\kappa_{i s} \mathrm{~F}_{2}^{i s}-\kappa_{i \nu} \mathrm{F}_{2}^{i \nu}\right),
\end{aligned}
$$

where the isoscalar and isovector parts are as in the Eqs. (A.46)-(A.49). Consequently, the neutral $\mathrm{Y}$-hyperon receives a small contribution from the electric coupling due to a finite $F_{1}$ contribution which vanishes in the real photon limit. 


\begin{tabular}{|c|c|c|c|c|c|c|}
\hline $\mathrm{p}:$ & $\begin{array}{l}F_{1}^{p} \\
F_{2}^{p}\end{array}$ & $\begin{array}{l}=1 \\
=1\end{array}$ & $\mathrm{~K}^{+}:$ & $\mathrm{F}_{\mathrm{K}^{+}}$ & $=$ & 1 \\
\hline$n, Y:$ & $\begin{array}{l}F_{1}^{n, Y}, \\
F_{2}^{n, Y}\end{array}$ & $\begin{array}{l}=0 \\
=1\end{array}$ & $\mathrm{~K}^{0}:$ & $F_{K^{0}}$ & $=$ & 0 \\
\hline $\begin{array}{l}N^{*}: \\
Y^{*}:\end{array}$ & $\begin{array}{l}\mathrm{F}_{\mathrm{N}^{*}} \\
\mathrm{~F}_{\mathrm{Y}^{*}}\end{array}$ & $\begin{array}{l}=1 \\
=1\end{array}$ & $\mathrm{~K}^{*}, \mathrm{~K}_{1}:$ & $\mathrm{F}_{\mathrm{K}^{*}}$ & $=$ & 1 \\
\hline
\end{tabular}

Table A.6 The real photon limit $\left(\mathrm{Q}^{2} \rightarrow 0\right)$ of the electromagnetic form factors at the different photo coupling vertices.

For the electromagnetic $\mathrm{N}^{*}$ and $\mathrm{Y}^{*}$ form factors, one should in principle use unique $\mathrm{Q}^{2}$-dependent effective coupling constants. As a matter of fact, at present there is hardly any knowledge about the shape of those functions for the $Y^{*}$ hyperon resonances and the $\mathrm{N}^{*}$ 's in the third resonance region. An extraction of these form factors from the data seems impossible at this stage. Therefore, we will adopt the Pauli form factor of the proton for charged resonances and the neutron one for neutral excited states. More precisely, we use $F_{2}^{p}$ at $\gamma^{*} N^{*} p$ and $F_{2}^{n}$ at $\gamma^{*} \gamma^{*} \Lambda$ and $\gamma^{*} Y^{*} \Sigma^{0}$ vertices.

For the sake of completeness, we report the real photon limits $\left(\mathrm{Q}^{2} \rightarrow 0\right)$ of all the form factors in Table A.6.

\section{A.3.2 Mesonic Form Factors}

For the $\gamma^{*} \mathrm{KK}$ vertex in the $\mathrm{t}$-channel, we have adopted two different parameterizations for the electromagnetic form factor. A first one is derived within a relativistic constituent quark model, based on the light-front formalism [100]. This form factor is refitted as the sum of a monopole and a dipole term by David et al. [13] and parameterized as:

$$
\mathrm{F}_{\mathrm{K}^{+}}\left(\mathrm{Q}^{2}\right)=\frac{\mathrm{a}}{1+\mathrm{Q}^{2} / \Lambda_{1}^{2}}+\frac{1-\mathrm{a}}{\left(1+\mathrm{Q}^{2} / \Lambda_{2}^{2}\right)^{2}},
$$


with $a=0.398, \wedge_{1}=0.642 \mathrm{GeV}$, and $\Lambda_{2}=1.386 \mathrm{GeV}$. An alternative monopole form for this form factor is proposed by Maris and Tandy [101] and reads:

$$
\mathrm{F}_{\mathrm{K}^{+}}\left(\mathrm{Q}^{2}\right)=\frac{1}{1+\mathrm{Q}^{2} / \Lambda^{2}}
$$

with $\wedge^{2}=0.61 \mathrm{GeV}^{2}$.

Although $\mathrm{K}^{0}$ is a neutral particle, the mass difference between the up and strange quark causes a nonzero form factor at finite $\mathrm{Q}^{2}$. At the $\gamma^{*} \mathrm{~K}^{0} \mathrm{~K}^{0}$ vertex, we use a form factor derived by Ito [102] within a vector meson dominance model:

$$
\mathrm{F}_{\mathrm{K}^{\circ}}\left(\mathrm{Q}^{2}\right)=\frac{-1 / 3}{1+\mathrm{Q}^{2} / \mathrm{m}_{\omega}^{2}}+\frac{1 / 3}{1+\mathrm{Q}^{2} / \mathrm{m}_{\phi}^{2}},
$$

with $\mathrm{m}_{\omega}=0.782 \mathrm{GeV}$ and $\mathrm{m}_{\phi}=1.019 \mathrm{GeV}$.

At the vector meson $\gamma^{*} \mathrm{~K}^{*+} \mathrm{K}^{+}, \gamma^{*} \mathrm{~K}_{1} \mathrm{~K}^{+}$and $\gamma^{*} \mathrm{~K}^{* 0} \mathrm{~K}^{0}$ vertices, David et al. [13] suggested a monopole transition form factor, parameterized as:

$$
\mathrm{F}_{\mathrm{K}^{*}}\left(\mathrm{Q}^{2}\right)=\frac{1}{1+\mathrm{Q}^{2} / \Lambda^{2}}
$$

with $\Lambda_{\mathrm{K}^{*+}}=0.95 \mathrm{GeV}, \Lambda_{\mathrm{K}_{1}}=0.55 \mathrm{GeV}$ and $\Lambda_{\mathrm{K}^{* 0}}=\mathrm{m}_{\rho}^{2}$. An alternative for the charged and neutral $\gamma^{*} K^{*} \mathrm{~K}$ transition form factors is given by Münz et al. [103] and is displayed in Fig. A.1.

The real photon limits of the different mesonic electromagnetic form factors are also summarized in Table A.6. All electroproduction calculations presented in this work are performed with the $\mathrm{F}_{\mathrm{K}^{+}}\left(\mathrm{Q}^{2}\right)$ and $\mathrm{F}_{\mathrm{K}^{*+}}\left(\mathrm{Q}^{2}\right)$ form factors of Eqs. (A.57) and (A.60), respectively. We have performed calculations with the other options (Eq. (A.58) and Fig. A.1) and observed deviations in the results. However, the lack of an extended strangeness electroproduction data base prevents us at present from drawing well founded conclusions for the different options. 


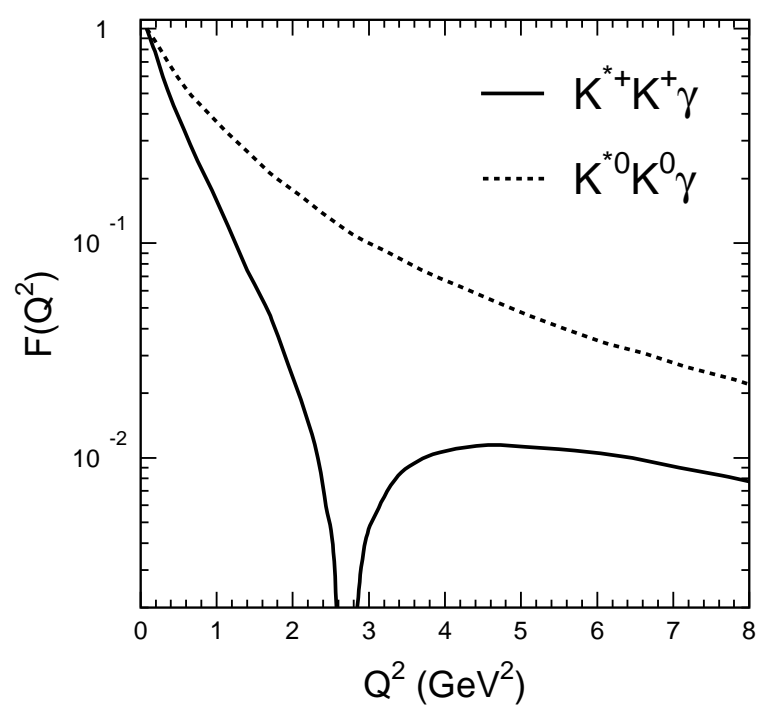

Figure A.1 The transition form factors for the vector meson decay $\mathrm{K}^{*+} \rightarrow \mathrm{K}^{+} \gamma$ and $\mathrm{K}^{* 0} \rightarrow$ $\mathrm{K}^{0} \gamma$ according to Ref. [103] 


\section{Appendix B}

\section{Minimization Procedure}

In this appendix, we spend a few words on a rather important technical aspect of the hadronic model calculations, namely the procedure which is required to obtain an optimum set of coupling constants.

As outlined previously, the isobar model is an effective field theory which lets the coupling constants undetermined. These numbers constitute an essential part of the physical information that can be extracted from the model calculations. There are two strategies which can be followed to deal with the information regarding the coupling constants. First, one can gather physically acceptable values for the coupling constants, either from experimental analyses or from calculations in e.g. a constituent quark model. Those values can be used as input parameters in the isobar model calculations. In this case, the isobar model serves as a test for those analyses or (quark) models. Another approach is to extract values for the free parameters by optimizing the isobar model calculations to the available data set. The extracted quantities can then be compared to e.g. quark model predictions. However, in both approaches the connection with quark model predictions has to be made with some reservations. Coupling constants computed within the context of quark models should not always be regarded as identical to those that are introduced in hadronic effective Lagrangian theories. For example, dressing mechanisms may somehow obscure this comparison.

In this work, we have adopted the second approach. In the determination of an optimum set of $n$ free parameters, we rely on a $\chi^{2}$ procedure. The objective function $\chi^{2}$ is defined as in Eq. (2.1) as a function of $n$ parameters. The best set of parame- 
ters is the one that produces the lowest $\chi^{2}$ value. In order to obtain this optimum set, one is forced to minimize $\chi^{2}$ in the $n$ dimensional parameter space. For the calculations presented in Chaps. 3 and $4, n$ roughly varies between 12 and 22. Although various minimization engines are available and described in literature, it goes without saying that finding a global minimum in a 22 dimensional space is not a trivial task.

To tackle the problem, we use a minimization algorithm known as simulated annealing and for example described in Ref. [104]. At the heart of the method is an analogy with thermodynamics, specifically with the way that liquids freeze and crystallize or metals cool and anneal. At high temperatures, the molecules of a liquid move freely with respect to one another. If the liquid is cooled slowly, thermal mobility is lost and the atoms are lining up. They form a pure crystal that is completely ordered over a distance up to billions of times the size of an individual atom. This crystal is the state of minimum free energy of the system. The amazing fact is that, for slowly cooled systems, nature is able to find this minimum energy state. In fact, if a liquid metal is cooled quickly or "quenched", it does not reach this state but rather ends up in a polycrystalline or amorphous state with a higher energy. So, the essence of the process is slow cooling, allowing time for redistribution of the atoms as they lose mobility. This is essential for ensuring that eventually a low free energy state will be reached.

Although the analogy is not perfect, the idea can be implemented starting form a downhill simplex algorithm. Therefore, a $(n+1)$ dimensional hypercube is constructed in the $n$ dimensional space. This hypercube is deformed by subsequent contractions and reflections of its vertex points in such a way that it results in a shrinkage of the hypercube into the lowest point. This idea is schematically made clear in Fig. B.1 for a 3-dimensional hypercube on a $\chi^{2}$ surface in a 2-dimensional parameter space. The hypercube indicated by the vertex points 1-2-3 is here the starting point. The highest (1) and lowest (3) point are determined. Then a reflection away from the highest point 1 results in a new hypercube 1'-2-3 with highest vertex point 2 and lowest point 1'. A new reflection away from 2 would result in an uphill step. Therefore, a contraction of vertex point 2 towards $1^{\prime}$ is a better option.

The procedure is well known to work excellently as long as it can be assured that there is only one (or, a few) minimum in the $n$ dimensional surface. How- 


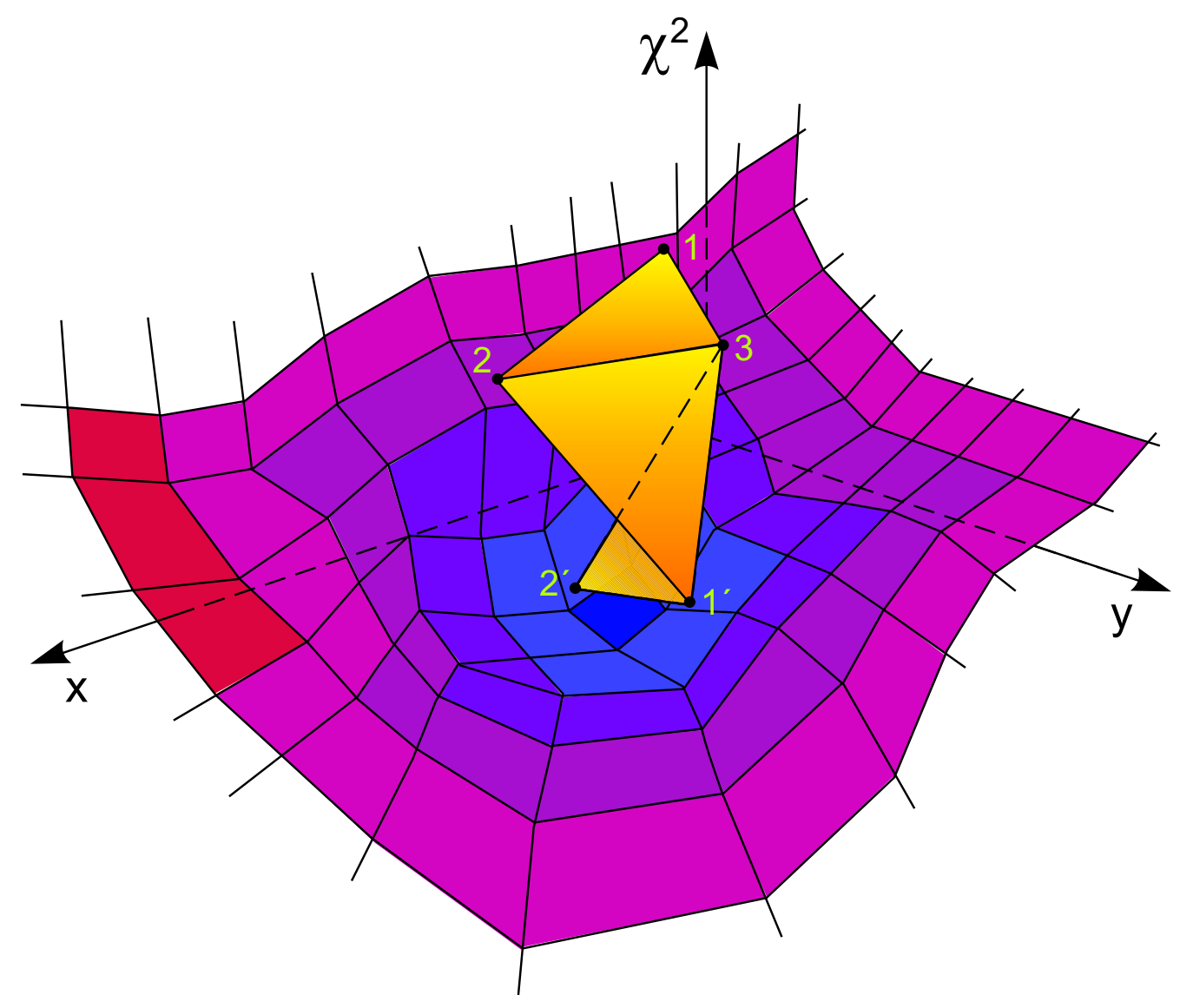

Figure B.1 A schematic picture of a $\chi^{2}$ surface in a 2-dimensional parameter space. The 3dimensional simplex hypercube is depicted and indicated by the vertex points 1-2-3. After a reflection away from the highest point 1 , the hypercube $1^{\prime}-2-3$ is obtained. A subsequent contraction of the highest point 2 towards $1^{\prime}$ is then the best option and results in the new hypercube $1^{\prime}-2^{\prime}-3$. 

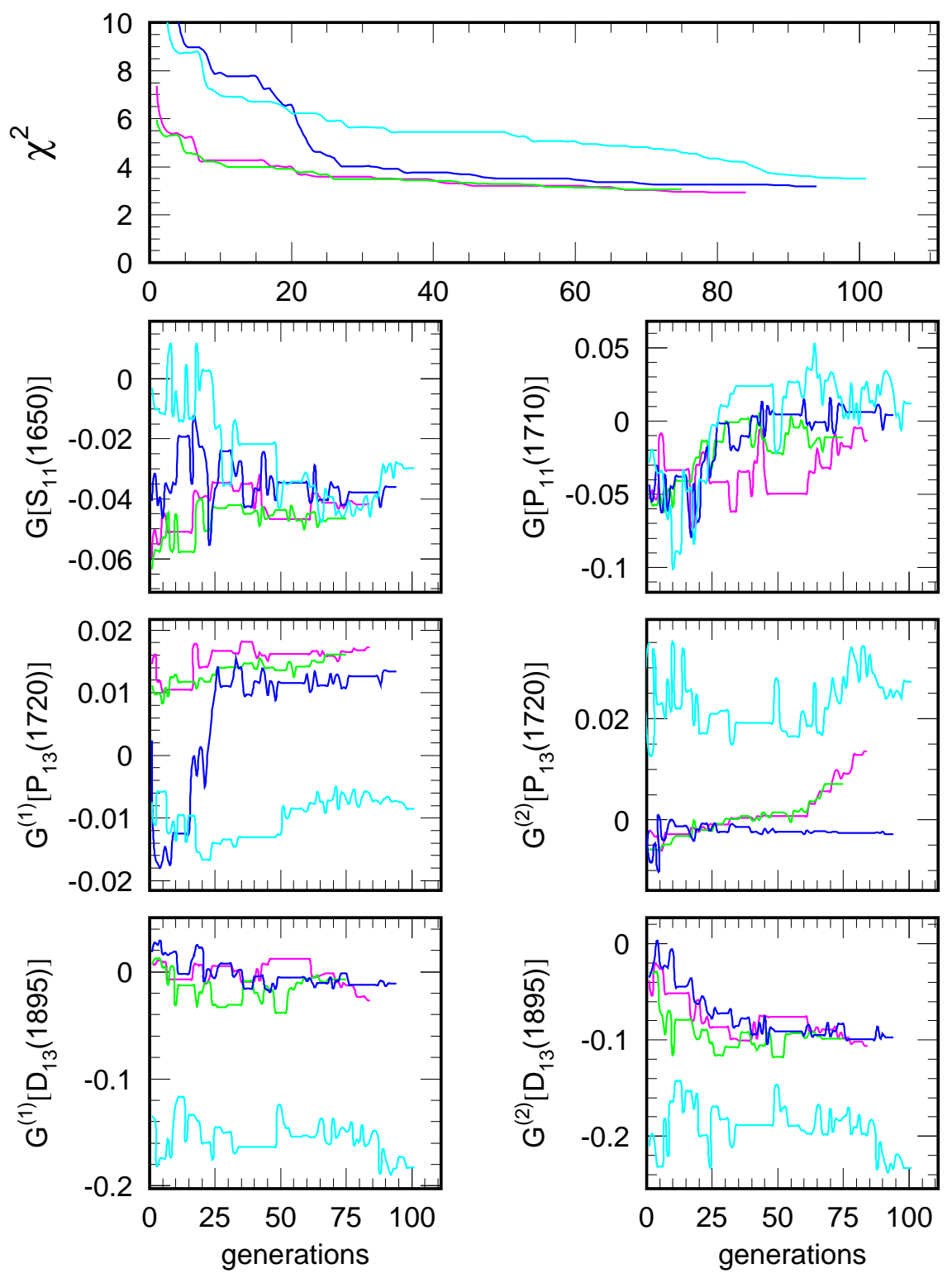

Figure B.2 Time evolution of the parameters for the $N^{*}$ resonances $S_{11}(1650), P_{11}(1710)$, $P_{13}(1720)$ and $D_{13}(1895)$ in the $p\left(\gamma, K^{+}\right) \wedge$ process. The results for four fitting procedures are indicated by different colors. For every fit, the optimum set is plotted after every generation. A generation accounts for about $2000 \chi^{2}$ evaluations at a fixed temperature. 
ever, it appears realistic to assume that this latter condition is not fully met in our calculations. So, to overcome this problem and to avoid getting trapped in one of the local minima, the downhill simplex method is combined with a Metropolis algorithm. The introduction of this Metropolis procedure basically implements the idea that a true downhill step is always accepted but sometimes also an uphill step, proportional to a temperature $\mathrm{T}$, is retained. Consequently, at high $\mathrm{T}$, the simplex hypercube is able to cross various local edges in this search of the global minimum. By decreasing temperature, the wells that can be crossed, become smaller and smaller. At every temperature, a fixed number $(\sim 2000)$ of $\chi^{2}$ evaluations are performed. A set of calculations at one $\mathrm{T}$ is called a generation. In the limit $\mathrm{T} \rightarrow 0$, the algorithm reduces to a simple downhill simplex procedure, which ends up in a local minimum. However, it is hoped that, in analogy to the annealing of a metal, the system has "cooled down" slowly enough and will arrive eventually in its global minimum energy state.

By minimizing $x^{2}$ in a large parameter space, it is important to make sure that eventually the absolute global minimum has been reached. In the course of performing the various fitting procedures, we have realized that within the framework of hadronic models, the $n$ dimensional $\chi^{2}$ surface has indeed numerous local minima. This becomes clear by plotting the evolution of the resonance parameters during the fitting procedure. An example of such a plot is given in Fig. B.2. This plot illustrates the time evolution of the minimization of the parameters for the $\mathrm{p}\left(\gamma, \mathrm{K}^{+}\right) \wedge$ process. For four different minimization "runs", indicated by different colors, the optimum set is plotted after every generation. From this figure, one can conclude that the $\chi^{2}$ surface has a very erratic behavior over the whole parameter space. Nevertheless, this plot contains some interesting information. As a first observation, one sees that the light blue calculation arrives at values for the coupling constants that strongly differ from the three other runs. Since the $\chi^{2}$ value for this particular calculation is also higher than the three other ones, we can conclude from this combined information that the run gets trapped in a local minimum. For the other three runs, comparable $\chi^{2}$ 's are obtained and also the coupling constants tend toward the same values. An exception is observed for the $\mathrm{G}^{(2)}$ parameter of the $P_{13}(1720)$ resonance. For this parameter, the three minimization calculations arrive at different optimal values. It appears that this coupling constant can vary over 
a large range, even flip sign, thereby producing comparable results for the quality of agreement with the data, expressed by $x^{2}$. This may be an indication that the presence of this resonance is rather unimportant for reproducing the energy and angular dependence of the data.

To conclude, we can state that most of the coupling constants arrive at values in a relatively small range, illustrating the effectiveness of the minimization procedure which we adopted. Nevertheless, the curves of Fig. B.2 clearly show that the results have to be regarded with the greatest care. To some extent, the minimization procedure is a numeric-technical problem. On the other hand, the erratic $\chi^{2}$ behavior for (some of) the extracted coupling constants is part of the physics of the isobar model which reproduces the data through a subtle interplay of different resonant and background contributions. It is to be expected, however, that the upcoming data for the various (polarization) observables will polish the wild variations on the $\chi^{2}$ surface and further constrain the identification of the various coupling constants. 


\section{Appendix C}

\section{Regge Scattering Amplitude}

In this appendix, we give a brief outline of the ideas lying behind Regge theory. For the sake of simplifying the mathematics, we focus on the scattering process $a+b \rightarrow c+d$, where the particles carry no spin and have equal masses $(m)$. The introduction of spin and non-equal masses is rather straightforward and does not alter the general conclusions.

Since total momentum is conserved in the $a+b \rightarrow c+d$ reaction, it is a commonly adopted technique to expand the amplitude into a Legendre series:

$$
M(s, t)=\sum_{l=0}^{\infty}(2 l+1) M_{l}(s) P_{l}\left(\cos \theta_{s}\right)
$$

where the partial wave amplitude $M_{l}(s)$ is given by:

$$
M_{l}(s)=\frac{1}{2} \int_{-1}^{+1} d \cos \theta_{s} M\left(s, t\left(\cos \theta_{s}\right)\right) P_{l}\left(\cos \theta_{s}\right), \quad l=0,1,2 \ldots
$$

The Mandelstam variables are defined in the standard manner:

$$
s=\left(p_{a}+p_{b}\right)^{2}, \quad t=\left(p_{a}-p_{c}\right)^{2}, \quad u=\left(p_{a}-p_{d}\right)^{2} .
$$

In the partial wave decomposition, $\cos \theta_{s}$ can be defined as a function of the Mandelstam variables $s$ and $t$ (or $u)$ :

$$
\cos \theta_{s}=1+\frac{2 t}{s-4 m^{2}}=-\left(1+\frac{2 u}{s-4 m^{2}}\right) .
$$

The Legendre expansion of Eq. (C.1) is valid in the whole physical region of the "direct" or s-channel process $a+b \rightarrow c+d$. This physical region is defined by the 


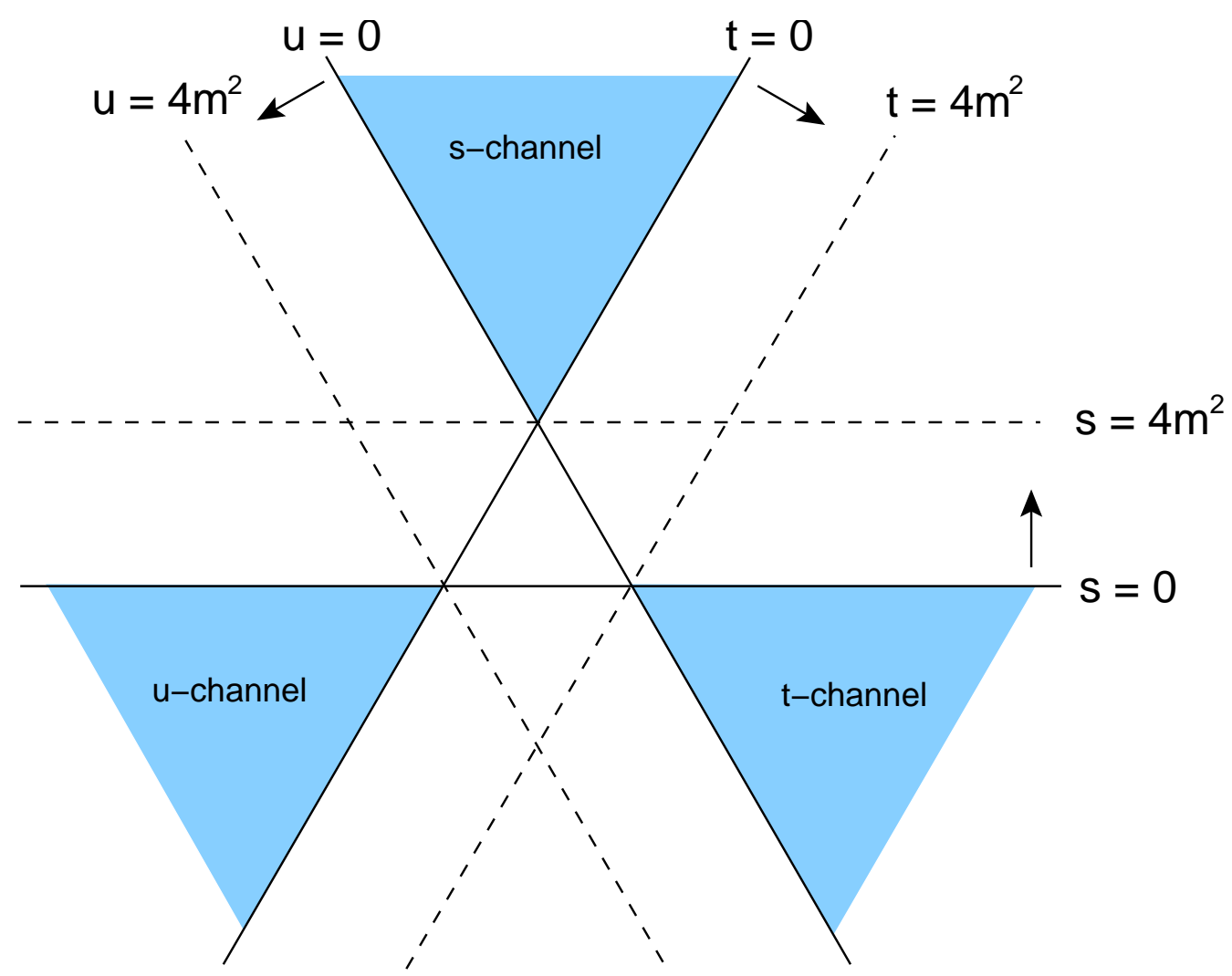

Figure C.1 The (s,t)-Mandelstam plane. The shaded areas indicate the physical regions for the $s-, t-$, and $u$-channel processes. The arrows indicate the direction of increasing Mandelstam variables. The dashed lines represent the respective thresholds at $4 \mathrm{~m}^{2}$. Note that also the $u$ variable is depicted although this is not an independent quantity in the $(\mathrm{s}, \mathrm{t})$-plane.

conditions that:

$$
\mathrm{s} \geq 4 \mathrm{~m}^{2}, \quad-1 \leq \cos \theta_{\mathrm{s}} \leq+1
$$

and is indicated in Fig. C.1. Despite these restrictions for the physical region of the direct process, the concept of crossing symmetry implies that the same amplitude $M(s, t)$ should also be able to describe the physics of the "crossed" process $a+\bar{c} \rightarrow$ $\bar{b}+d$. Obviously, for the crossed process, $M(s, t)$ has to be applied in another region of the $(s, t)$-Mandelstam plane. The direct and crossed processes are schematically depicted in Fig. C.2. For the crossed, or so called t-channel process, an analogous 


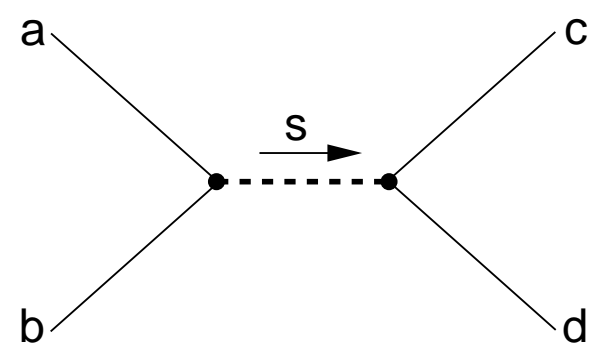

direct s-channel

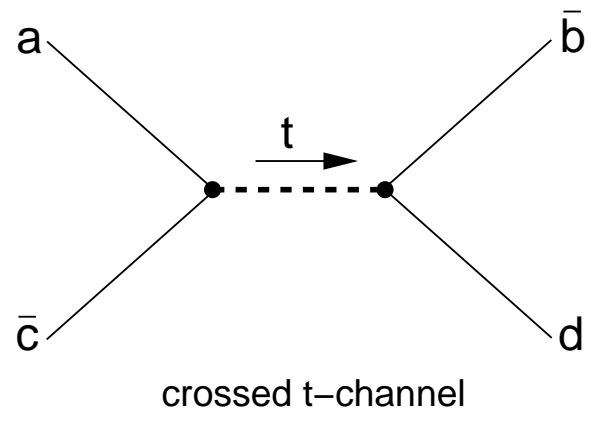

Figure C.2 The direct (left) and crossed (right) process for the $a+b \rightarrow c+d$ reaction. The arrow indicates the exchanged four momentum.

decomposition as in Eq. (C.1) can be constructed:

$$
M(s, t)=\sum_{l=0}^{\infty}(2 l+1) M_{l}(t) P_{l}\left(\cos \theta_{t}\right)
$$

with:

$$
\cos \theta_{t}=1+\frac{2 s}{t-4 m^{2}}=-\left(1+\frac{2 u}{t-4 m^{2}}\right) .
$$

In the forthcoming derivations, we will often use the shorthand notation $z \equiv \cos \theta_{\mathrm{t}}$.

Let us now consider the specific case where the $t$-channel is strongly dominated by the exchange of one single pole with spin $l$ and mass $m_{l}$. This dominance can, for example, be motivated by the experimental observation of a resonant structure in the $t$-channel process, or, by the diffractive behavior in the corresponding direct s-channel reaction. According to the decomposition of Eq. (C.6), the amplitude is dominated by the corresponding partial wave $M_{l}(t)$ and one can write:

$$
M(s, t) \sim \frac{g_{a c}(t) g_{b d}(t)}{t-m_{l}^{2}} P_{l}\left(\cos \theta_{t}\right),
$$

with $g_{a c}(t)$ and $g_{b d}(t)$ the corresponding vertex functions. Since the amplitude $M(s, t)$ of Eq. (C.8) contains the relevant physical ingredients of the crossed $t$ channel process $a+\bar{c} \rightarrow \bar{b}+d$, one could also try to utilize this expression for the description of the direct s-channel process, as suggested by crossing symmetry.

It can be shown, however, that such an extrapolation has to be handled with the greatest care since one can run into unphysical situations. To make this clear, we will restrict ourselves to the high $s$ limit since this greatly simplifies the mathematical derivations. For example, there holds $\cos \theta_{t} \gg 1$ according to Eq. (C.7). Note 
that in this regime, $\cos \theta_{t}$ is not a physical quantity anymore. As a result, we can make use of the asymptotic behavior of the Legendre function, which reads:

$$
\mathrm{P}_{\mathrm{l}}(z) \stackrel{z \rightarrow \infty}{\longrightarrow} \begin{cases}\frac{1}{\sqrt{\pi}} \frac{\Gamma(\mathrm{l}+1 / 2)}{\Gamma(\mathrm{l}+1)}(2 z)^{\mathrm{l}}, & \operatorname{Re}\{l\} \geq-1 / 2, \\ \frac{1}{\sqrt{\pi}} \frac{\Gamma(-\mathrm{l}-1 / 2)}{\Gamma(-\mathrm{l})}(2 z)^{-\mathrm{l}-1}, & \operatorname{Re}\{l\} \leq-1 / 2,\end{cases}
$$

where $\Gamma$ is the gamma function. Consequently, for the exchange of a spin-l particle in the high s limit, one has:

$$
P_{l}\left(\cos \theta_{t}\right) \sim\left(\cos \theta_{t}\right)^{l}
$$

and, according to (C.7) and (C.8):

$$
M(s, t) \sim s^{l} .
$$

From the optical theorem it is known that $\sigma_{\text {tot }} \sim \operatorname{Im}\{M(s, t=0)\} / s$. With the relation (C.11), the following s-dependence for the total cross section arises:

$$
\sigma_{\text {tot }} \sim s^{l-1}
$$

It is clear that this asymptotic behavior gives rise to unphysical divergences for $l>1$. Indeed, it was proven by Froissart [105] on the basis of unitarity, that the total cross section at high energies is constrained by:

$$
\sigma_{\text {tot }} \stackrel{s \rightarrow \infty}{\leq} \mathcal{C}(\log s)^{2}
$$

This complication can be attributed to the partial wave expansion. Under the condition of the occurrence of one dominant pole, the expansion gives rise to Eq. (C.8). This expression is legitimate in the t-channel physical region. However, attempts have been made to employ the same expression in the physical plane of the direct $s$-channel where the convergence of the partial wave expansion can no longer be guaranteed.

In Refs. [106,107], Lehmann and Martin showed that the convergence of a partial wave expansion of the type (C.1) or (C.6) can only be guaranteed for $s \geq 4 \mathrm{~m}^{2}$ and $t \geq 4 \mathrm{~m}^{2}$, respectively and inside the so called Lehmann-Martin ellipse. This ellipse defines the convergence boundaries for the partial wave expansion in the $\operatorname{complex} \cos \theta$ plane for fixed $s$ or $t$. The Lehmann-Martin ellipse is shown in 


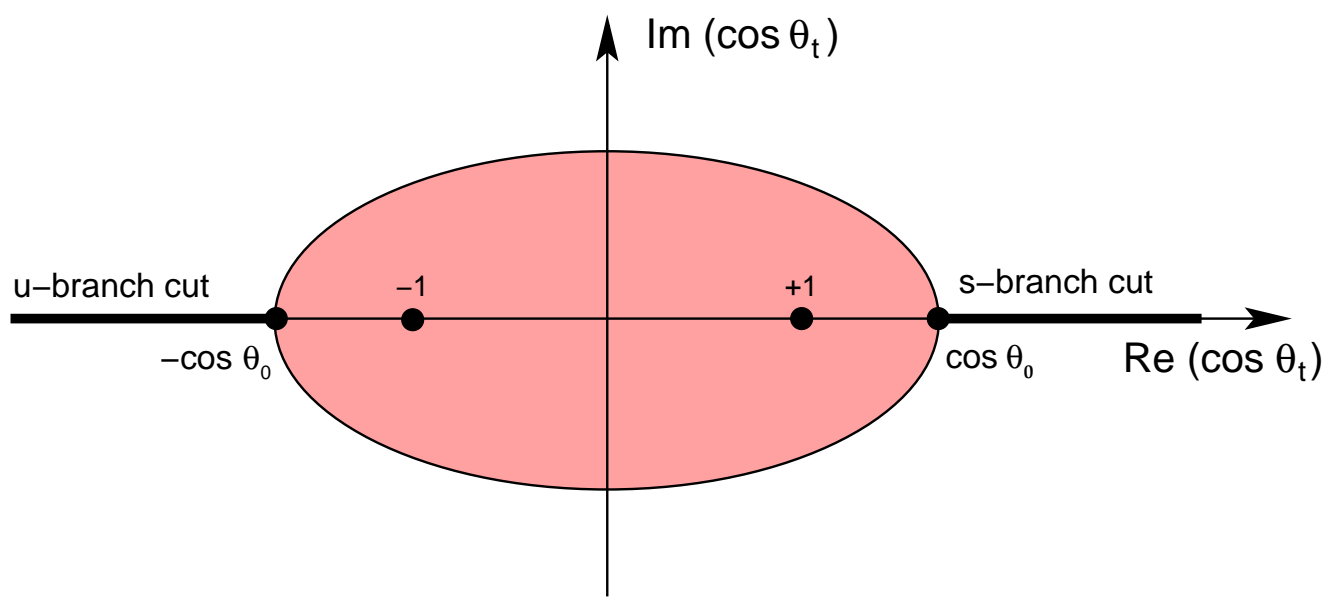

Figure C.3 The Lehmann-Martin ellipse in the complex $\cos \theta_{t}$ plane, for the equal mass $\mathrm{t}$-channel scattering process. The figure also shows the $\mathrm{s}$ - and $u$-channel branch cuts.

Fig. C.3 for the t-channel process for the specific case that all particles participating in the reaction have equal masses. It has foci at \pm 1 and a large axis which is determined by:

$$
\cos \theta_{0}=1+\frac{8 m^{2}}{t-4 m^{2}}
$$

Note that this value $\cos \theta_{0}$, according to Eq. (C.7), corresponds to the branch points of $M(s, t)$ in the complex $\cos \theta_{t}$ plane which occur at $s=4 \mathrm{~m}^{2}$ and $u=4 \mathrm{~m}^{2}$. In extrapolating the partial-wave expansion of Eq. (C.8) towards the physical region of the direct s-channel process, one goes beyond the boundaries of the LehmannMartin ellipse in the $\cos \theta_{\mathrm{t}}$ plane and it cannot be guaranteed that the expansion will converge.

A method to master this difficulty, is to sum over all $t$-channel partial waves first and, in a next step, extrapolate beyond the convergence region. Thereby, the summation must be carried out in such a way that a good analytic function is obtained which can be used outside the Lehmann-Martin ellipse. This idea constitutes the basis of Regge theory. A plausible technique which succeeds in summing the partial waves up to infinity is the transformation of this sum into a contour integral in the complex plane of the summation variable. To obtain this, we introduce a complex angular momentum $l$, which will be denoted by $\lambda$. According to the 


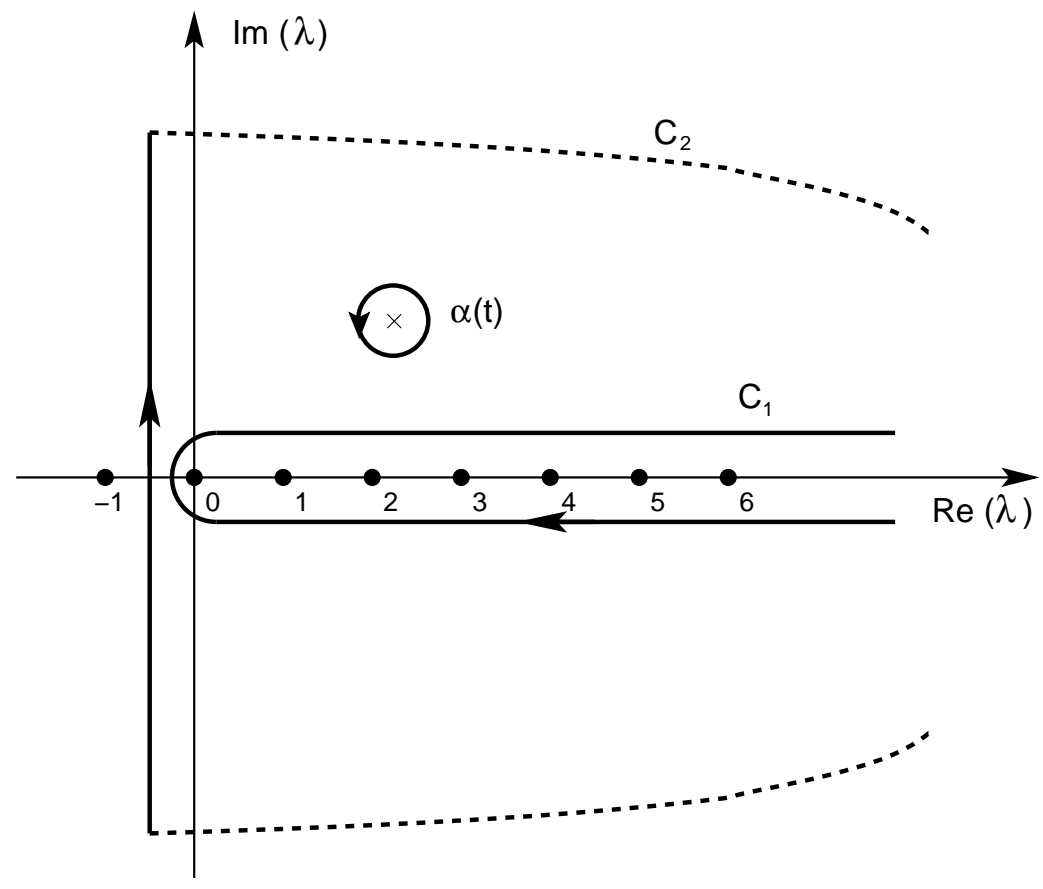

Figure C.4 The contours $C_{1}$ and $C_{2}$ in the complex angular momentum plane. The poles at the real axis and at $\alpha(t)$ are indicated.

residue theorem, Eq. (C.6) can in this complex momentum plane be written as:

$$
M(s, t)=-\frac{1}{2 i} \oint_{C_{1}} d \lambda \frac{(2 \lambda+1) M_{\lambda}(t) P_{\lambda}\left(-\cos \theta_{t}\right)}{\sin (\pi \lambda)} .
$$

This expression is known as the Sommerfeld-Watson transformation. The poles are produced by the $[\sin (\pi \lambda)]^{-1}$ since:

$$
\sin (\pi \lambda) \stackrel{\lambda \rightarrow l}{\longrightarrow}(-1)^{\lambda}(\lambda-l) \pi .
$$

The contour $C_{1}$ is shown in Fig. C.4. Necessary condition for the SommerfeldWatson transformation is the postulate of maximal analyticity of the second kind which requires that $M_{\lambda}(t)$ has only isolated singularities in the $\lambda$-plane. This theorem can not be proven on the basis of fundamental principles. The theory, however, seems to be confirmed by strong interaction models [75] and, more importantly, does not lead to expressions which contradict the available experimental information.

To simplify the integral in Eq. (C.15), it it will be necessary to deform the contour to infinity. Therefore the condition $M_{\lambda}(t) \rightarrow 0$ for $|\lambda| \gg 0$ is required. To verify this 
convergence, we start from a dispersion relation for $M(s, t)$, based on the Cauchy integral formula, that includes the branch cuts of the amplitude in the complex $\cos \theta_{\mathrm{t}}$ plane:

$$
\begin{aligned}
M\left(s\left(\cos \theta_{t}\right), t\right)= & \text { pole terms }+\frac{1}{\pi} \int_{\cos \theta_{0}}^{+\infty} d z \frac{D_{s}(z, t)}{z-\cos \theta_{t}} \\
& -\frac{1}{\pi} \int_{-\infty}^{-\cos \theta_{0}} d z \frac{D_{u}(z, t)}{z-\cos \theta_{t}} .
\end{aligned}
$$

The discontinuity functions along the branch cuts are defined as:

$$
\begin{aligned}
D_{s}(z, t) & =\lim _{\varepsilon \rightarrow 0} \frac{1}{2 i}(M(s+i \varepsilon, t, u)-M(s-i \varepsilon, t, u)), \\
D_{u}(z, t) & =\lim _{\varepsilon \rightarrow 0} \frac{1}{2 i}(M(s, t, u+i \varepsilon)-M(s, t, u-i \varepsilon)) .
\end{aligned}
$$

The dispersion relation (C.17) can be inserted in the definition of the partial wave amplitude:

$$
\begin{aligned}
M_{\lambda}(t) & =\frac{1}{2} \int_{-1}^{+1} \mathrm{~d} \cos \theta_{t} M\left(s\left(\cos \theta_{t}\right), t\right) P_{\lambda}\left(\cos \theta_{t}\right) \\
& =\frac{1}{2} \int_{-1}^{+1} d \cos \theta_{t} \frac{1}{\pi} \int_{\cos \theta_{0}}^{+\infty} d z P_{\lambda}\left(\cos \theta_{t}\right)\left[\frac{D_{s}(z, t)}{z-\cos \theta_{t}}+\frac{D_{u}(-z, t)}{z+\cos \theta_{t}}\right]
\end{aligned}
$$

With the aid of the Legendre function of the $2^{\text {nd }}$ kind, defined as:

$$
\mathrm{Q}_{\lambda}(z)=\frac{1}{2} \int_{-1}^{+1} \mathrm{~d} z^{\prime} \frac{\mathrm{P}_{\lambda}\left(z^{\prime}\right)}{z-z^{\prime}}
$$

and the interchange of the integration variables, one eventually arrives at the FroissartGribov projection:

$$
M_{\lambda}(t)=\frac{1}{\pi} \int_{\cos \theta_{0}}^{+\infty} d z Q_{\lambda}(z)\left[D_{s}(z, t)+(-1)^{\lambda} D_{u}(-z, t)\right] .
$$

We can now make use of the asymptotic behavior of the Legendre function of the $2^{\text {nd }}$ kind:

$$
\mathrm{Q}_{\lambda}(z) \stackrel{|\lambda| \rightarrow \infty}{\longrightarrow} \lambda^{-1 / 2} e^{-(\lambda+1 / 2) 1\left(q z+\sqrt{z^{2}+1}\right)}
$$

which ensures that $M_{\lambda}(t)$ in Eq. (C.21) converges for large $\lambda$ except for the factor $(-1)^{\lambda}$. As a solution to this problem, it turns out to be appropriate to introduce the concept of signature $\zeta= \pm$ corresponding with two distinct types of partial waves:

$$
\mathrm{M}_{\lambda}^{\zeta= \pm}(\mathrm{t})=\frac{1}{\pi} \int_{\cos \theta_{0}}^{+\infty} \mathrm{d} z \mathrm{Q}_{\lambda}(z)\left[\mathrm{D}_{\mathrm{s}}(z, \mathrm{t})+\zeta \mathrm{D}_{\mathrm{u}}(-z, \mathrm{t})\right]
$$


For each of the two possibilities, $M_{\lambda}^{\zeta}(t)$ now converges for $|\lambda| \rightarrow \infty$. The connection to the physical partial wave amplitudes is straightforward and reads:

$$
\begin{array}{ll}
M_{\lambda}(t)=M_{\lambda}^{+}(t), & \lambda=0,2,4 \ldots \\
M_{\lambda}(t)=M_{\lambda}^{-}(t), & \lambda=1,3,5 \ldots
\end{array}
$$

As such, it is proven that the amplitude $M_{\lambda}(t)$ has the required convergence properties $\left(M_{\lambda}(t) \rightarrow 0\right.$ for $\left.|\lambda| \gg 0\right)$, as long as the amplitudes of a well defined signature are treated separately.

The contour $C_{1}$ of the Sommerfeld-Watson transformation of Eq. (C.15) can now be deformed into the contour $\mathrm{C}_{2}$ as depicted in Fig. C.4 since the contributions at infinity will vanish. Through deforming the contour, also other singularities of the amplitude $M_{\lambda}^{\zeta}(t)$ have to be taken into account. It was Regge who postulated that the only singularities of the amplitude are the poles in the complex plane at $\lambda=\alpha_{i}(t)$. In the vicinity of such a pole $\alpha_{i}(t)$, the amplitude $M_{\lambda}^{\zeta}(t)$ takes on the form:

$$
M_{\lambda}^{\zeta}(t) \stackrel{\lambda \rightarrow \alpha_{i}(t)}{\longrightarrow} \frac{\beta_{i}(t)}{\lambda-\alpha_{i}(t)}
$$

with $\beta_{i}(t)$ the residue of the amplitude at the pole. Assuming that $M_{\lambda}^{\zeta}(t)$ has only one pole in the complex $\lambda$-plane (for fixed $t$ ), the Sommerfeld-Watson representation of the amplitude becomes:

$$
\begin{aligned}
M^{\zeta}(s, t)= & -\frac{1}{2 i} \int_{-1 / 2-i \infty}^{-1 / 2+i \infty} d \lambda \frac{(2 \lambda+1) M_{\lambda}^{\zeta}(t) P_{\lambda}\left(-\cos \theta_{t}\right)}{\sin (\pi \lambda)} \\
& -\frac{2 \pi i}{2 i}(2 \alpha(t)+1) \frac{\beta(t)}{\sin (\pi \alpha(t))} P_{\alpha(t)}\left(-\cos \theta_{t}\right) .
\end{aligned}
$$

The first term is called the "background integral". Due to the asymptotic behavior (C.9) of $\mathrm{P}_{l}(z)$, the background integral behaves like $s^{-1 / 2}$ for high $s$. Consequently, this term can be neglected in the high s limit and the second term, stemming from the pole $\alpha(t)$, represents the dominant contribution to the amplitude.

To ensure that $M_{\lambda}(t) \rightarrow 0$ for $|\lambda| \gg 0$, the Sommerfeld-Watson amplitude is derived for a specific signature. The total amplitude can now be obtained by adding the even $(\zeta=+)$ and the odd $(\zeta=-)$ parts. From Eq. (C.23), it is clear that $M_{\lambda}^{\zeta}(t)$ has well defined symmetry properties under the exchange of the $s$ and $u$ Mandel- 
stam variables. The following relations are easily proven:

$$
\begin{aligned}
(s, u) & \longleftrightarrow(u, s), \\
z & \longleftrightarrow-z, \\
M_{\lambda}^{+}(t) & \longleftrightarrow M_{\lambda}^{+}(t), \\
M_{\lambda}^{-}(t) & \longleftrightarrow-M_{\lambda}^{-}(t) .
\end{aligned}
$$

In order to impose these symmetry properties on the total scattering amplitude, it is appropriate to write the functional dependence of the amplitudes of defined signature of Eq. (C.26) as $M^{\zeta}(z, t)$ instead of $M^{\zeta}(s, t)$ and then combine them as:

$$
M(s, t)=\frac{1}{2}\left[M^{+}(z, t)+M^{+}(-z, t)+M^{-}(z, t)-M^{-}(-z, t)\right] .
$$

Building up the total amplitude in this way, it is ensured that the correct symmetry properties are included. Assuming that the "background integral" in Eq. (C.26) can be neglected, we obtain the following expression for the amplitude:

$$
M^{\zeta}(s, t)=-\pi(2 \alpha(t)+1) \frac{\beta(t)}{\sin (\pi \alpha(t))} \frac{1}{2}\left[P_{\alpha(t)}\left(-\cos \theta_{t}\right)+\zeta P_{\alpha(t)}\left(\cos \theta_{t}\right)\right]
$$

The sum of the two Legendre functions in this expressions can be rewritten as:

$$
\left[\left(1+\zeta e^{-i \pi \alpha(t)}\right) P_{\alpha(t)}\left(-\cos \theta_{t}\right)-\zeta \frac{2}{\pi} \sin (\pi \alpha(t)) Q_{\alpha(t)}\left(-\cos \theta_{t}\right)\right]
$$

The term proportional to $\mathrm{Q}_{\alpha(\mathrm{t})}(z)$ can be neglected in the high $s$ limit due to its asymptotic behavior:

$$
\mathrm{Q}_{\alpha}(z) \stackrel{z \rightarrow \infty}{\longrightarrow} \sqrt{\pi} \frac{\Gamma(\alpha+1)}{\Gamma\left(\alpha+\frac{3}{2}\right)}(2 z)^{-\alpha-1} .
$$

As such, our final result for the high s limit of the scattering amplitude reads:

$$
M^{\zeta}(s, t)=-\pi(2 \alpha(t)+1) \frac{\beta(t)}{\sin (\pi \alpha(t))} \frac{1+\zeta e^{-i \pi \alpha(t)}}{2} P_{\alpha(t)}\left(-\cos \theta_{t}\right)
$$

Note that $\alpha(t)$ is defined as the pole of the partial wave $M_{\lambda}^{\zeta}(t)$. This means that for a certain value of $t$, the situation $\alpha(t)=l$, corresponds to a physical particle or resonance with $m=\sqrt{t}$ and spin $l$. In the standard partial-wave expansion of Eq. (C.6), the exchange of all those poles is taken into account on a pole by pole 
basis. With the aid of the Sommerfeld-Watson transformation, we have obtained a closed expression for $M(s, t)$. Here, the various $t$-channel poles are still incorporated, though, by means of the $\sin (\pi \alpha(t))$ function. Every time $\alpha(t)$ passes through a positive integer value, the $\sin (\pi \alpha(t))$ produces a pole in the expression of Eq. (C.32). Phenomenologically it has been observed that $\alpha(t)$, which makes the connection between the spin $(=l)$ and the pole position in the variable $t\left(=\mathrm{m}^{2}\right)$, can be parameterized as a linear function. In should be noted here, that in the $s$-channel process, those physical materializations of $\alpha(t)$ are never reached since $t \leq 0$. To the contrary, it is precisely the exchange of the trajectory $\alpha(t)$ as a whole, representing a class of particles, that contributes.

The expression (C.32) can further be simplified if we restrict ourselves to the so called Regge limit: high $s$ and small negative $t(s \gg$ and $|t| \ll)$. Consequently, $\alpha(t)$ is small and the following expression for the Regge scattering amplitude can be directly obtained from (C.32):

$$
M_{\text {Regge }}^{\zeta}(s, t)=C\left(\frac{s}{s_{0}}\right)^{\alpha(t)} \frac{\beta(t)}{\sin (\pi \alpha(t))} \frac{1+\zeta e^{-i \pi \alpha(t)}}{2} \frac{1}{\Gamma(\alpha(t)+1)} .
$$

Here, use is made of the expression (C.9) for the high $z$ behavior of the $P_{\alpha(t)}(z)$ and the fact that, $\Gamma(\alpha(t)+1 / 2) \simeq \sqrt{\pi}$ and $(2 \alpha(t)+1) \simeq 1$, for small $\alpha(t)$. The gamma function in the denominator approaches 1 but ensures that non-physical poles, generated by the function $\sin (\pi \alpha(t))$ at larger negative values of $\alpha(t)$ cannot contribute. The constant $C$ which is introduced, has to be defined in connection with the residue function $\beta(t)$. The scale factor $s_{0}$ is arbitrarily fixed at $1 \mathrm{GeV}^{2}$. Note that the high $s$ dependence of the Regge amplitude (C.33) is in accordance with the Froissart bound of Eq. (C.13) as long as $\alpha(t=0) \leq 1$.

The above scattering amplitude was derived for spin-less particles in an equal mass hypothesis. To extend the theory to particles with non-equal masses, the relevant physical regions in the Mandelstam plane (Fig. C.1) and the LehmannMartin ellipse (Fig. C.3) become distorted. For example, the physical region of the $s$-channel process is not bounded anymore by the conditions $t=0, u=0$ but receives $s$-dependent thresholds $t_{\min }(s)$ and $u_{\min }(s)$ [75]. When the external particles do carry spin, this will reflect itself in the residue function $\beta(t)$ that will contain more complicated vertex functions.

To conclude this section, we summarize that we have constructed an amplitude 
for a general $a+b \rightarrow c+d$ reaction, which is valid in the so called Regge limit ( $\$$ and $|t| \ll)$. It is ensured that the amplitude is unitary and analytic. The amplitude is not constructed on the basis of the exchange mechanism of isolated poles but on the exchange of an entire family of particles which contribute to the process as a whole. 



\section{Appendix D}

\section{The Veneziano Model}

Back in 1968, a relatively simple and phenomenological model to describe $\pi^{+} \pi^{-} \rightarrow$ $\pi^{+} \pi^{-}$scattering was developed by Veneziano [81]. In a traditional Feynman diagram picture, this process is dominated by $\rho$ and $f_{2}$ meson exchange. In developing his model, Veneziano was guided by the duality requirement for the crossing symmetric process. The duality hypothesis states that, when computing a scattering amplitude, it does not really matter whether the summation is performed over all the s-channel or all the $t$-channel poles $[11,75]$. Or, formally:

$$
M(s, t)=\sum_{n}^{\infty} \frac{\beta_{n}(s, t)}{s-s_{n}}=\sum_{m}^{\infty} \frac{\beta_{m}(s, t)}{t-t_{m}} .
$$

Here, $\beta_{i}$ denotes the residue at the respective poles. As a consequence, special care has to be exercised when calculating $M(s, t)$ with a limited set of both $s-$ and $\mathrm{t}$-channel poles. Indeed, chances are real that certain poles will be doubly counted.

Veneziano suggested a form for the reaction amplitude which contains both the $s$ - and t-channel poles, at the same time respecting the duality hypothesis. The poles are parameterized by means of a linear trajectory $\alpha$ in such a manner that a pole occurs if $\alpha$ becomes positive and integer. Note that this procedure bears a strong resemblance with the one that is adopted when parameterizing Regge trajectories. The amplitude form put forward by Veneziano reads:

$$
M(s, t)=V(s, t) \equiv g \frac{\Gamma(1-\alpha(s)) \Gamma(1-\alpha(t))}{\Gamma(2-\alpha(s)-\alpha(t))} .
$$

Remind that $\Gamma(z)$ is an analytic function over the entire complex plane, save for the poles $z=0,-1,-2 \ldots$ where it possesses simple poles. Note that the function $V(s, t)$ 
is crossing symmetric under the interchange of the variables $s$ and $t$, as required for the $\pi^{+} \pi^{-} \rightarrow \pi^{+} \pi^{-}$process. The gamma functions in the nominator produce the required poles in the $s$ and $t$ variables for the exchange of entire $\rho$ and $f_{2}$ trajectories. The gamma function in the denominator takes out the doubly counted poles when both $\alpha(s)$ and $\alpha(t)$ take on integer values. Its presence guarantees that the duality hypothesis is respected.

Let us now explore the high s limit of the amplitude of Eq. (D.2). Therefore, we can make use of the asymptotic behavior of the gamma function:

$$
\Gamma(z) \stackrel{z \rightarrow \infty}{\longrightarrow}(2 \pi)^{1 / 2} e^{-z} z^{(z-1 / 2)}, \quad \text { if }|\arg z|<\pi,
$$

which is valid except for a wedge about the negative real z-axis. From this expression, it is straightforward to derive:

$$
\frac{\Gamma(z+\mathrm{a})}{\Gamma(z+\mathrm{b})} \stackrel{z \rightarrow \infty}{\longrightarrow} z^{\mathrm{a}-\mathrm{b}} .
$$

This asymptotic behavior, in combination with the reflection formula:

$$
\Gamma(1-z)=\frac{\pi}{\Gamma(z) \sin (\pi z)},
$$

can be used to rewrite the Veneziano amplitude of Eq. (D.2) in the high s limit as:

$$
V(s, t) \stackrel{s \rightarrow \infty}{\longrightarrow} g \frac{\pi(-\alpha(s))^{\alpha(t)-1}}{\Gamma(\alpha(t)) \sin (\pi \alpha(t))} .
$$

For linear trajectories $\alpha(s)$, which are phenomenologically observed as long as $s>$ 0 , this expression can be cast in the familiar (spin-1 exchange) Regge amplitude:

$$
V(s, t) \stackrel{s \rightarrow \infty}{\longrightarrow} g \frac{\pi\left(\alpha^{\prime} s\right)^{\alpha(t)-1} e^{-i \pi(\alpha(t)-1)}}{\Gamma(\alpha(t)) \sin (\pi \alpha(t))} .
$$

The scale factor $s_{0}$, introduced in Eq. (5.1) can be identified here in a natural way as $s_{0}=1 / \alpha^{\prime}$. The expression in (D.7) is quite a remarkable result with some striking properties. First, the Regge behavior in the high $s$ limit is retrieved in a very natural way. Assuming a linear trajectory $\alpha(s)$, one arrives at a rotating phase in $\alpha(t)$. On the basis of Eq. (5.10), this is indeed expected for the $\pi^{+} \pi^{-} \rightarrow \pi^{+} \pi^{-}$process where the degenerated $\rho$ and $f_{2}$ meson trajectories are exchanged. Further on, the Veneziano amplitude in the form (D.2) is manifestly crossing symmetric and, consequently, possesses the same poles and Regge behavior in s and t. Accordingly, 
within his relatively simple model, Veneziano succeeded to incorporate some fundamental physical concepts.

So far, the Veneziano amplitude $V(s, t)$ has been derived for the fully crossing symmetric $\pi^{+} \pi^{-}$scattering process, dominated by vector meson exchanges. For processes where the crossing symmetry under the interchange of the Mandelstam variables is not manifestly present, one could think of writing the total amplitude as a sum of three Veneziano contributions in order to take into account the different $\mathrm{s}-, \mathrm{t}-$ and $\mathrm{u}$-channel exchange contributions:

$$
M(s, t)=V(s, t)+V(s, u)+V(t, u)
$$

The most general Veneziano function in $s$ and $t$ reads:

$$
\mathrm{V}(\mathrm{s}, \mathrm{t})=\mathrm{g} \frac{\Gamma(\mathrm{a}-\alpha(\mathrm{s})) \Gamma(\mathrm{b}-\alpha(\mathrm{t}))}{\Gamma(\mathrm{a}+\mathrm{b}-\alpha(\mathrm{s})-\alpha(\mathrm{t}))}
$$

and analogous expressions exist for $V(s, u)$ and $V(t, u)$. Here, we have introduced $a, b$ and $c$ as the spin values of the first materialization of the trajectory in each Mandelstam channel. The sum (D.8) over the three Veneziano amplitudes can be worked out with the aid of the Eq. (D.5) and the consistency condition [84]:

$$
\alpha(s)+\alpha(t)+\alpha(u)=\Phi
$$

This condition is the trajectory equivalent of the relation $s+t+u=\sum_{i} m_{i}^{2}$, relating the three Mandelstam variables in a two particle scattering process. After some straightforward analytic manipulations and the particular choice of $\Phi=a+b+c-1$, the amplitude $M(s, t)$ can be rewritten as:

$$
\begin{aligned}
& M(s, t)=g \frac{\Gamma(b-\alpha(t)) \Gamma(c-\alpha(u))}{\Gamma(\alpha(s)-a+1)} \\
& \quad \times\left[1+e^{-i \pi(\alpha(t)-b}+\sin (\pi(\alpha(t)-b)) \frac{1+e^{i \pi(\alpha(s)-a)}}{\sin (\pi(\alpha(s)-a))}\right] .
\end{aligned}
$$

With the use of Eqs. (D.4) and (D.5), it can be shown that the factor in front again exhibits the correct Regge behavior in the high s limit:

$$
\mathrm{g} \frac{\Gamma(\mathrm{b}-\alpha(\mathrm{t})) \Gamma(\mathrm{c}-\alpha(\mathrm{u}))}{\Gamma(\alpha(\mathrm{s})-\mathrm{a}+1)} \stackrel{\mathrm{s} \rightarrow \infty}{\longrightarrow} \mathrm{g} \frac{-\pi(\alpha(\mathrm{s}))^{\alpha(\mathrm{t})-\mathrm{b}}}{\Gamma(\alpha(\mathrm{t})-\mathrm{b}+1) \sin (\pi(\alpha(\mathrm{t})-\mathrm{b}))} .
$$


At this stage, a remark concerning the phase factor of Eq. (D.11) is in order. Comparing Eq. (D.11) to the original Regge amplitude for a trajectory exchange in the t-channel, an extra term:

$$
\sin (\pi \alpha(t)) \frac{1+e^{i \pi \alpha(s)}}{\sin (\pi \alpha(s))},
$$

emerges in the phase factor of the Veneziano amplitude. This term finds its origin in the introduction of the $s$ - and $u$-channel poles which is a peculiar property of the Veneziano approach. The spin shifts $-a$ and $-b$ are omitted in the expression (D.13) in order to simplify the notation. In the numerical calculations, they are always taken into account according to the Eqs. (5.7) and (5.16). 


\section{Bibliography}

[1] K. Wilson, "Problems in Physics with Many Scales of Length," Scientific American 241, 140 (1979).

[2] P. Stassart and F. Stancu, Phys. Rev. D 42, 1521 (1990).

[3] L. Glosman and D. Riska, Phys. Rep. 268, 264 (1996).

[4] S. Capstick and W. Roberts, Phys. Rev. D 58, 074011 (1998).

[5] U. Löring, B. Metsch, and H. Petry, Eur. Phys. J. A 10, 395 (2001).

[6] T. Kuo, Phys. Rev. 130, 1537 (1963).

[7] H. Thom, Phys. Rev. 151, 1322 (1966).

[8] F. Renard and Y. Renard, Nucl. Phys. B 25, 490 (1970).

[9] R. Adelseck, C. Bennhold, and L. Wright, Phys. Rev. C 32, 1681 (1985).

[10] R. Adelseck and B. Saghai, Phys. Rev. C 42, 108 (1990).

[11] R. Williams, C.-R. Ji, and S. Cotanch, Phys. Rev. C 46, 1617 (1992).

[12] T. Mart, C. Bennhold, and C. Hyde-Wright, Phys. Rev. C 51, 1074(R) (1995).

[13] J. David, C. Fayard, G. Lamot, and B. Saghai, Phys. Rev. C 53, 2613 (1996).

[14] M. Cheoun, B. Han, B. Yu, and I.-T. Cheon, Phys. Rev. C 54, 1811 (1996).

[15] T. Mizutani, C. Fayard, G. Lamot, and B. Saghai, Phys. Rev. C 58, 75 (1998).

[16] M. Guidal, J.-M. Laget, and M. Vanderhaeghen, Nucl. Phys. A 627, 645 (1997).

[17] P. Donoho and R. Walker, Phys. Rev. 112, 981 (1958).

[18] T. Fujii et al., Phys. Rev. D 2, 439 (1970).

[19] R.L. Anderson et al., Phys. Rev. Lett. 9, 131 (1962). 
[20] K.H. Althoff et al., Nucl. Phys. B 137, 269 (1978).

[21] A. Bleckmann et al., Z. Phys. 239, 1 (1970).

[22] M. Bockhorst et al., Z. Phys. C 63, 37 (1994).

[23] M.Q. Tran et al., Phys. Lett. B 445, 20 (1998), http://lisa12.physik.unibonn.de/saphir/klks.txt.

[24] S. Goers et al., Phys. Lett. B 464, 331 (1999), http://lisa12.physik.unibonn.de/saphir/web_values_k0si.txt.

[25] T. Feuster and U. Mosel, Phys. Rev. C 59, 460 (1999).

[26] S. Hsiao, D. Lu, and S. Yang, Phys. Rev. C 61, 068201 (2000).

[27] T. Mart and C. Bennhold, Phys. Rev. C 61, (R)012201 (2000).

[28] W.-T. Chiang, F. Tabakin, T.-S. Lee, and B. Saghai, Phys. Lett. B 517, 101 (2001).

[29] B. Saghai, nucl-th/0105001 (2001).

[30] S. Janssen, J. Ryckebusch, W. Van Nespen, D. Debruyne, and T. Van Cauteren, Eur. Phys. J. A 11, 105 (2001).

[31] S. Janssen, J. Ryckebusch, D. Debruyne, and T. Van Cauteren, Phys. Rev. C 65, 015201 (2002).

[32] S. Janssen, J. Ryckebusch, D. Debruyne, and T. Van Cauteren, Phys. Rev. C submitted (2002), nucl-th/0202074.

[33] B. Saghai and Z. Li, Eur. Phys. J. A 11, 217 (2001).

[34] R. Alkofer, S. Ahlig, C. Fischer, and M. .Oettel, Nucl. Phys. A 680, 70c (2001).

[35] Q. Zhao, Z. Li, and C. Bennhold, Phys. Rev. C 58, 2393 (1998).

[36] J. Ramon, N. Kaiser, S. Wetzel, and W. Weise, Nucl. Phys. A 672, 249 (2000).

[37] E. Oset, J. Nacher, E. Marco, J. Oller, J. Pelaez, A. Ramos, and H. Toki, Prog. Part. Nucl. Phys. 44, 213 (2000). 
[38] M. Vanderhaeghen, M. Guidal, and J.-M. Laget, Phys. Rev. C 57, 1454 (1998).

[39] M. Guidal, J.-M. Laget, and M. Vanderhaeghen, Phys. Rev. C 61, 025204 (2000).

[40] C. Bennhold and L. Wright, Phys. Rev. C 36, 438 (1987).

[41] B. Han, M. Cheoun, K. Kim, and I.-T. Cheon, Nucl. Phys. A 691, 713 (2001).

[42] Z. Li and F. Close, Phys. Rev. D 42, 2207 (1990).

[43] B. Pearce and B. Jennings, Nucl. Phys. A 528, 655 (1991).

[44] H. Haberzettl, Phys. Rev. C 56, 2041 (1997).

[45] H. Haberzettl, C. Bennhold, T. Mart, and T. Feuster, Phys. Rev. C 58, (R)40 (1998).

[46] R. Davidson and R. Workman, Phys. Rev. C 63, 025210 (2001).

[47] J. de Swart, Rev. Mod. Phys. 35, 916 (1963).

[48] J. Donoghue and B. Holstein, Phys. Rev. D 25, 2015 (1982).

[49] M. Vanderhaeghen, K. Heyde, J. Ryckebusch, and M. Waroquier, Nucl. Phys. A 595, 219 (1995).

[50] M. Benmerrouche, N. Mukhopadhyay, and J. Zhang, Phys. Rev. D 51, 3237 (1995).

[51] A. Gasparian, J. Haidenbauer, C. Hanhart, L. Kondratyuk, and J. Speth, Nucl. Phys. A 684, 397c (2001).

[52] B. Holzenkamp, K. Holinde, and J. Speth, Nucl. Phys. A 500, 485 (1989).

[53] A. D. Martin, Nucl. Phys.B 179, 33 (1981).

[54] T. Mart, Phys. Rev. C 62, 038201 (2000).

[55] K.-H. Glander, In Proceedings of the NSTAR 2001 Workshop on The Physics of Excited Nucleons, D. Drechsel and L. Tiator, eds., p. 381 (World Scientific, New Jersey, 2001). 
[56] F. Mandl and G. Shaw, Quantum Field Theory (John Wiley \& Sons, Chichester, 1984).

[57] I. Baker, A. Donnachie, and J. Storrow, Nucl. Phys. B 95, 347 (1975).

[58] Particle Data Group, D.E. Groom et al., Eur. Phys. J. C 15, 1 (2000).

[59] A. D'Angelo, In Proceedings of the NSTAR 2001 Workshop on The Physics of Excited Nucleons, D. Drechsel and L. Tiator, eds., p. 347 (World Scientific, New Jersey, 2001).

[60] R. Zegers, In Proceedings of the EMI2001 Symposium, (Osaka, Japan, 2001).

[61] K. Ohta, Phys. Rev. C 40, 1335 (1989).

[62] R. Davidson and R. Workman, Phys. Rev. C 63, 058201 (2001).

[63] R. Arndt, R. Workman, Z. Li, and L. Roper, Phys. Rev. C 42, 1853 (1990).

[64] T. Mart and C. Bennhold, Nucl. Phys. A 639, 237c (1998).

[65] B. Saghai, Nucl. Phys. A 639, 217c (1998).

[66] B. Raue, In Proceedings of the NSTAR 2001 Workshop on The Physics of Excited Nucleons, D. Drechsel and L. Tiator, eds., p. 373 (World Scientific, New Jersey, 2001).

[67] G. Knöchlein, D. Drechsel, and L. Tiator, Z. Phys. A 352, 327 (1995).

[68] F. Gross and D. Riska, Phys. Rev. C 36, 1928 (1987).

[69] S. Nozawa and T.-S. Lee, Nucl. Phys. A 513, 511 (1990).

[70] G. Niculescu et al., Phys. Rev. Lett. 81, 1805 (1998).

[71] C.J. Bebek et al., Phys. Rev. Lett. 32, 21 (1974).

[72] C.J. Bebek et al., Phys. Rev. D 15, 594 (1977).

[73] C.N. Brown et al., Phys. Rev. Lett. 28, 1086 (1972).

[74] T. Regge, Nuovo Cimento 14, 951 (1959). 
[75] P. Collins, An introduction to Regge theory and high energy physics (Cambridge University Press, Cambridge, 1977).

[76] R. Omnès, Ann. Rev. Nucl. Sci. 16, 263 (1966).

[77] J. Forshaw and D. Ross, Quantum Chromodynamics and the Pomeron (Cambridge University Press, Cambridge, 1997).

[78] J. Storrow, Rep. Prog. Phys. 50, 1229 (1987).

[79] M. Guidal, Ph.D. thesis, DAPNIA, CEA/Saclay, Gif-sur-Yvette Cèdex, France, 1997.

[80] A.M. Boyarski et al., Phys. Rev. Lett. 22, 1131 (1969).

[81] G. Veneziano, Nuovo Cimento 57A, 190 (1968).

[82] M. Ahmad, Fayyazuddin, and Riazuddin, Phys. Rev. Lett. 23, 504 (1969).

[83] R. Brower and M. Halpern, Phys. Rev. 182, 1779 (1969).

[84] J. W. Alcock, Y. A. Chao, and R. E. Cutkosky, Nucl. Phys.B 84, 503 (1975).

[85] D. Sivers, S. Brodsky, and R. Blanckenbecler, Phys. Rep. 23C, 1 (1976).

[86] P. Collins and P. Kearney, Z. Phys. C 22, 277 (1984).

[87] M. Sergeenko, Z. Phys. C 64, 315 (1994).

[88] R. Schumacher, Nucl. Phys. A 663, 440c (2000).

[89] T. Feuster and U. Mosel, Nucl. Phys. A 612, 375 (1997).

[90] M. Peskin and D. Schroeder, An Introduction to Quantum Field Theory (Perseys Books, Reading, Massachusetts, 1995).

[91] M. Benmerrouche, R. Davidson, and N. Mukhopadhyay, Phys. Rev. C 39, 2339 (1989).

[92] D. Drechsel, O. Hanstein, S. Kamalov, and L. Tiator, Nucl. Phys. A 645, 145 (1999). 
[93] D. H. Perkins, Introduction to High Energy Physics, 4th ed. (Cambridge University Press, Cambridge, 2000).

[94] T. Theussl, R. Wagenbrunn, B. Desplanques, and W. Plessas, Eur. Phys. J. A 12, 91 (2001).

[95] P. Singer and G. Miller, Phys. Rev. D 33, 141 (1986).

[96] M. Gari and W. Krümpelmann, Phys. Lett. B 173, 10 (1986).

[97] M. Gari and W. Krümpelmann, Phys. Rev. D 45, 1817 (1992).

[98] M. Gari and W. Krümpelmann, Phys. Lett. B 274, 159 (1992), erratum, Phys. Lett. B 282, 483 (1992).

[99] E. L. Lomon, Phys. Rev.C 64, 035204 (2001).

[100] F. Cardarelli, I. L. Grach, I. M. Narodetskii, E. Pace, G. Salmé, and S. Simula, Phys. Rev. D 53, 6682 (1996).

[101] P. Maris and P. Tandy, Phys. Rev. C 62, 055204 (2000).

[102] C. Bennhold, H. Ito, and T. Mart, In Proceedings of the 7th International Conference on the Structure of Baryons, p. 323 (Santa Fe, New Mexico, 1995).

[103] C. Münz, J. Resag, B. Metsch, and H. Petry, Phys. Rev. C 52, 2110 (1995).

[104] W. Press, S. Teukolsky, W. Vetterling, and B. Flannery, Numerical Recipes in C, The art of Scientific Computing, 2nd ed. (Cambridge University Press, Cambridge, 1992).

[105] M. Froissart, Phys. Rev. 123, 1053 (1961).

[106] H. Lehmann, Nuovo Cimento 10, 579 (1958).

[107] A. Martin, Nuovo Cimento 42, 930 (1966). 


\section{Nederlandse Samenvatting}

\section{Inleiding}

Een in het oog springend kenmerk van de natuur is de grote diversiteit aan lengteschalen die wordt waargenomen [1]. Hierbij valt op dat een bepaalde structuur of een bepaald systeem op een zekere lengteschaal beschreven wordt door fysische principes die op een kleinere of grotere schaal totaal onbruikbaar zijn. Een microscopisch model, bijvoorbeeld, kan de correcte bouwstenen bevatten, maar blijkt dikwijls te falen wanneer het eigenschappen op een veel grotere schaal moet beschrijven. Een beschrijving van het systeem zal immers vele malen efficiënter zijn wanneer de grootheden in acht worden genomen die op dat niveau of op die schaal belangrijk zijn. Voor de fysica is het dus cruciaal om de juiste vrijheidsgraden van een probleem te identificeren. Daarbij moeten uiteraard ook de onderlinge verbanden tussen de vrijheidsgraden op de verschillende niveaus begrepen worden. Enkel dan kan het systeem als volledig gekarakteriseerd beschouwd worden.

In de nucleaire en subatomaire fysica wordt men geconfronteerd met een gelijkaardige probleemstelling in de zin dat de fundamentele bouwstenen zich niet manifesteren als de aangewezen vrijheidsgraden om de veelheid aan fenomenen te verklaren die worden waargenomen in de subatomaire wereld. In de jaren '60 kwam men tot de vaststelling dat de honderden waargenomen hadronen geen fundamentele bouwstenen van de materie vormen. Deze hadronen, die structuur bleken te bevatten, konden echter worden geordend door het bestaan van een nieuwe beperkte set deeltjes op een nog kleiner niveau te veronderstellen. Deze vaststelling was het startschot voor de ontwikkeling van een totaal nieuwe kijk op de sterke wisselwerking in termen van quark- en gluonvrijheidsgraden. De fundamentele vergelijkingen voor de beschrijving van deze quarks en gluonen zijn sindsdien samengevat in de Quantum Chromo Dynamica (QCD). Hoewel de basisvergelijkingen van deze QCD-theorie redelijk eenvoudig zijn, zijn ze enkel oplosbaar onder omstandigheden waarbij de individuele quarks en gluonen als vrije objecten kunnen worden beschouwd. Deze voorwaarde doet zich echter enkel voor onder zeer 
extreme omstandigheden zoals bv. gecreërd in gespecialiseerde deeltjesversnellers. Er zijn immers sterke aanwijzingen dat in onze natuur enkel welbepaalde conglomeraten van quarks en gluonen kunnen overleven. Dit is het zogenaamde "confinement" principe. Gevolg is dat QCD de juiste vrijheidsgraden en voorspellingen levert in het zeer hoge energiegebied, maar zo goed als onbruikbaar is om hadronen en hun onderlinge reacties bij lagere energieën te beschrijven. Quarks en gluonen blijken hier niet meer de juiste vrijheidsgraden te vormen.

Een fundamentele uitdaging voor de medium-energie fysica is een brug te slaan tussen de nucleaire fysica waar hadronen, zoals protonen, neutronen en pionen de fundamentele vrijheidsgraden vormen en de QCD-theorie van de quarks en gluonen. Het is vandaag immers allesbehalve duidelijk hoe quarks en gluonen zich juist samenstellen tot de geobserveerde hadronen en wat de juiste aard en drijvende kracht van "confinement" is. Een veelbelovende manier om hierin beter inzicht te verkrijgen en de structuur van het nucleon te ontrafelen is het bestuderen van zijn excitatie spectrum. De resonanties van het nucleon (en hadronen in het algemeen) reflecteren immers een onderliggende structuur en moeten op één of andere manier de signatuur dragen van hun quark- en gluonbouwstenen. De zoektocht naar de structuur en de aard van de nucleonresonanties bekleedt dan ook een vooraanstaande plaats in het huidige onderzoek. Elektron- en fotonfaciliteiten zoals CEBAF, ELSA, MAMI, SPring-8, LEGS, MIT-Bates... leveren momenteel experimentele data van ongeziene kwaliteit. Eén van de grote uitdagingen voor de medium-energie fysica is dan ook uit deze data informatie te vergaren over specifieke eigenschappen van de resonanties en dit op een zo modelonafhankelijke manier als mogelijk.

De laatste decennia werd reeds heel wat informatie over de aangeslagen nucleontoestanden bekomen. Hierbij werden de meeste gegevens verzameld uit pionproductie en pion geïnduceerde reacties waarbij de resonanties als intermediaire toestanden gecreëerd worden. Reeds lang is men zich echter bewust van het feit dat men op deze manier slechts een gedeelte van het spectrum zichtbaar kan maken. Deze veronderstelling wordt bovendien ondersteund door de voorspellingen van (constituent) quark modellen die inderdaad veel meer resonanties voorspellen dan er tot nu toe experimenteel waargenomen zijn in de pionsector [2-5]. Onmiddellijk rijst een vraag die verder onderzoek verdient. Kunnen die overige of "mis- 
sende" resonanties in andere, niet-pionische kanalen geobserveerd worden of is er iets grondig mis met de (constituent) quark modellen die de basis vormen voor de beschrijving van de structuur van het nucleon?

Een mogelijke reactie om deze "missende" resonantietoestanden van het nucleon te onderzoeken zijn vreemdheidsproductie reacties. Er wordt immers algemeen aangenomen dat de aanwezigheid van een vreemd quark anti-quark paar in de reactie, naast de op en neer quarks uit de pion-nucleon sector, nieuwe inzichten kan bieden die kunnen leiden tot een beter begrip van het spectrum van het nucleon. Eén van de meest eenvoudige reacties die zo een $s \bar{s}$ quark anti-quark paar betrekt in het reactieproces, is de elektromagnetische productie van kaonen, kort genoteerd als $p(\gamma, K) Y$, waarin $Y=\Lambda, \Sigma$. De theoretische beschrijving en interpretatie van dit soort reacties vormt het onderwerp van deze thesis.

De modellering van $p(\gamma, \mathrm{K}) \mathrm{Y}$ valt uiteen volgens twee verschillende benaderingen van het probleem. Een eerste aanpak vertrekt van een volledig hadronische beschrijving. Hierbij worden de hadronen en hun aangeslagen toestanden als effectieve deeltjes beschouwd. Naar deze methode wordt in de literatuur gerefereerd als "isobare modellen" $[13,26,45]$. Anderzijds kan men vertrekken vanuit een partonische benadering. Hierbij worden QCD-geïnspireerde vrijheidsgraden aangewend om de reactie te beschrijven. Een mogelijke manier om dit te implementeren wordt geboden door Regge-theorie [16]. In dit werk wordt zowel een isobaar als een Regge geïnspireerd model voorgesteld.

\section{Isobaar Model voor $p(\gamma, \mathrm{K}) \mathrm{Y}$ Reacties}

Steunend op een effectieve-veldentheorie werd een model opgesteld om de vreemdheidsproductie reacties te beschrijven in termen van hadronische vrijheidsgraden. De hadronen en hun geëxciteerde toestanden worden beschouwd als effectieve velden met specifieke eigenschappen. De theoretische beschrijving valt uiteen in twee fundamenteel verschillende delen die echter onlosmakelijk met elkaar verbonden zijn: het resonante stuk en de achtergrond.

Het resonante gedeelte wordt opgebouwd door alle nucleonresonanties die via het s-kanaal tot het proces bijdragen. In dit werk werd veel aandacht besteed aan het bepalen van de dominante resonantiebijdragen in de verschillende vreemdheidsproductie reacties. In het $\mathrm{K}^{+} \wedge$ kanaal werd vastgesteld dat de vier nucleonre- 
sonanties $S_{11}(1650), P_{11}(1710), P_{13}(1720)$ en $D_{13}(1895)$ een belangrijke rol spelen in de reactiedynamica en in staat zijn om de recente SAPHIR data $[23,24]$ goed te beschrijven. In de $K \Sigma$ reacties werden drie nucleonresonanties $\left(S_{11}(1650), P_{11}(1710)\right.$, $\left.\mathrm{P}_{13}(1720)\right)$ en twee $\Delta^{*}$ toestanden $\left(S_{31}(1900)\right.$ en $\left.\mathrm{P}_{31}(1910)\right)$ geïdentificeerd als dominante intermediaire deeltjes.

In de zoektocht naar de resonante bijdragen werd veel aandacht besteed aan het fenomeen van de missende resonanties. Recent werd door de George Washington groep gerapporteerd dat een $\mathrm{D}_{13}$ resonantie met een massa rond $1.9 \mathrm{GeV}$ de beschrijving van de $\mathrm{K}^{+} \wedge$ data gevoelig kan verbeteren [27]. De structuur in de totale werkzame doorsnede rond $1.5 \mathrm{GeV}$ foton lab energie kon hiermee duidelijk verklaard worden. Hierbij moet opgemerkt worden dat deze $\mathrm{D}_{13}(1895)$ toestand nog niet werd waargenomen in pion-nucleon verstrooiing of pion productie reacties. Daarentegen werd deze nucleonexcitatie wel voorspeld door constituentquark modelberekeningen van Capstick en Roberts [4] met bovendien een significante vervalbreedte in de vreemdheidskanalen. Als dusdanig is deze $\mathrm{D}_{13}$ resonantie een goede kandidaat voor één van de missende resonanties. Onze modelberekeningen bevestigen de vaststelling van de G. Washington groep. De theoretische beschrijving van de $\mathrm{K}^{+} \wedge$ data verbetert gevoelig. In dit werk werd echter vastgesteld dat ook andere nucleon resonanties in staat zijn om de structuur rond $\omega_{\text {lab }}=$ $1.5 \mathrm{GeV}$ in de data te verklaren. Bovendien werd de verbetering niet waargenomen in de beschrijving van de $K \Sigma$ processen. Uit dit alles blijkt dat de identificatie van de juiste quantumgetallen en een verdere karakterisatie van deze missende resonanties met de grootste omzichtigheid behandeld moet worden. Verder onderzoek is onontbeerlijk en de aanwezigheid van deze toestand(en) moet zeker nog bevestigd worden in andere observabelen en andere reactiekanalen.

Naast het bepalen van de dominante resonanties werd in dit werk ook aandacht besteed aan de parametrisatie van de achtergrond. Deze achtergrond wordt in het isobaar model opgebouwd door alle niet-resonante termen (Born termen en $t$ - en u-kanaal resonanties) en blijkt een zeer belangrijke rol te spelen in de modellering van het proces. Vertrekkend van een exacte SU(3)-symmetrie in de quark-smaken ruimte, kan men, steunend op de goedgekende $\pi \mathrm{N}$ koppeling, de parameters van de Born termen bepalen [47]. Het is echter geweten dat de SU(3)-smaken symmetrie gebroken is. Over de juiste grootte van de breking bestaat nog onduidelijkheid, 
maar dit laat toch toe om realistische grenzen op te stellen waarbinnen de koppelingsconstanten van de Born termen kunnen variëren. Wanneer met deze waarden de bijdrage van de Born termen tot de werkzame doorsneden wordt berekend, worden resultaten bekomen die een factor vier tot vijf boven de waargenomen data liggen. Deze discrepantie geeft duidelijk aan dat bijkomende ingrediënten aan de theoretische beschrijving moeten toegevoegd worden om de bijdragen van de Born termen te reduceren. In dit werk werden drie mogelijke methodes voorgesteld om dit te bereiken.

- Model A: Een eerste methode steunt op het invoeren van "zachte" hadronische vormfactoren. Het is algemeen bekend dat vormfactoren een amplitude kunnen afvlakken. Wanneer echter de bijdrage van de Born termen moet gereduceerd worden tot een realistisch niveau, blijkt dat in de vormfactoren een cutoff massa van de orde van de kaon massa moet gebruikt worden. Op deze manier domineren de vormfactoren echter de fysica van het proces over heel het reactiegebied.

- Model B: Een tweede manier bestaat erin om enkele $Y^{*}$ hyperon resonanties in het $u$-kanaal te introduceren. Er werd vastgesteld dat deze $\Lambda^{*}$ en $\Sigma^{*}$ resonanties destructief kunnen interfereren met de andere termen van de achtergrond. Op deze manier wordt de sterkte van de Born termen gereduceerd tot een accepteerbaar niveau. Hierbij moet één opmerking gemaakt worden. De koppelingsconstanten die nodig zijn om dit effect te bekomen blijken groot te zijn in vergelijking met de corresponderende koppelingsconstanten van de nucleonresonanties. Om dit probleem uit te klaren, hebben we berekeningen uitgevoerd met een zevental hyperon resonanties in het $u$-kanaal. Dezelfde destructieve interferenties werden waargenomen, maar nu met kleinere individuele hyperon koppelingsconstanten. Op basis van deze bevindingen concluderen we dat de twee geïntroduceerde hyperon resonanties zouden kunnen beschouwd worden als effectieve deeltjes voor een grotere set intermediaire toestanden in het $\mathrm{u}$-kanaal.

- Model C: Tenslotte kan men de SU(3)-relaties, die de koppelingsconstanten van de Born termen vast leggen, gewoon negeren en de parameters volledig vrij laten in de fittingprocedures. Het blijkt echter dat voor de koppelings- 
constanten van de Born termen waarden worden bekomen die slechts $10 \%$ bedragen van wat op basis van exacte SU(3) berekeningen wordt verwacht. Dit zou duiden op een zeer sterke symmetriebreking.

Alle drie de schema's, voorgesteld om de achtergrond op te bouwen, slagen erin een goede beschrijving te geven van de SAPHIR dataset, bestaande uit differentiële en totale werkzame doorsneden en recoil polarisatie asymmetrieën.

Zonder twijfel is er echter met de parametrisatie van de achtergrond ook een zekere modelafhankelijkheid geïntroduceerd. Deze modelafhankelijkheid werd vervolgens grondig onderzocht in zowel de geëxtraheerde resonantieparameters als in berekende voorspellingen voor observabelen die nog niet gemeten zijn. In sommige gevallen werd aangetoond dat de effecten van de verschillende achtergrond parametrisaties niet te verwaarlozen zijn en grondig verschillende resultaten kunnen opleveren.

De modellen opgesteld voor reële foton productie, werden ook geëxtrapoleerd in het $\mathrm{Q}^{2} \neq 0$ gebied van de elektron geïnduceerde reacties. Vergelijking met de (schaarse) $\mathrm{K}^{+} \wedge$ data [70] bracht aan het licht dat vooral de longitudinale responsfunctie, een grootheid die niet waargenomen wordt door reële fotonen, als een zeer geschikte test fungeert voor de opgestelde theoretische modellen. Aan de hand van deze $\mathrm{K}^{+} \Lambda$ data moeten we concluderen dat de introductie van zachte vormfactoren (model A) en een sterke SU(3) symmetriebreking op het niveau van de koppelingsconstanten (model C) achtergronden creëren die niet in staat zijn om de geobserveerde sterkte in de longitudinale respons te verklaren. Enkel de introductie van hyperon resonanties in het $u$-kanaal is in staat om de nodige sterkte te genereren. Deze grote discrepantie tussen de verschillende modellen in voorspellingen voor de longitudinale responsfunctie werd niet waargenomen in de $K \Sigma$ kanalen.

Als besluit moet er dus gesteld worden dat een volledige en gefundeerde karakterisatie van de $\mathrm{p}\left(\gamma, \mathrm{K}^{+}\right) \wedge$ en $\mathrm{p}(\gamma, \mathrm{K}) \Sigma$ reacties aan de hand van een uitgebreide en accurate dataset als die van de SAPHIR collaboratie nog niet mogelijk is. Er is echter goede hoop dat de nieuwe data voor verscheidene polarisatieobservabelen en elektron geïnduceerde responsfuncties die momenteel geanalyseerd worden $[55,59,60,66]$, in staat zullen zijn om de theoretische beschrijving verder te verbeteren en de reactie dynamica van de vreemdheidsproductie reacties te ontrafelen. 


\section{Regge-Model voor $p(\gamma, K) Y$ Reacties}

Naast het isobaar model werd er ook een beschrijving van de vreemdheidsproductie reacties voorgesteld die gebaseerd is op Regge-theorie [74,75]. Regge-theorie slaagt erin om op een natuurlijke wijze een verband te leggen tussen partonische vrijheidsgraden en de hadronen die worden waargenomen. Zodoende biedt deze theorie een unieke mogelijkheid om vanuit een partonisch beeld voor de hadronen, observabelen te beschrijven.

In de literatuur werd enkele jaren geleden een model voorgesteld dat er in slaagt om een zeer goede beschrijving te geven van de hoge energie $p\left(\gamma, K^{+}\right) \wedge$ en $p\left(\gamma, K^{+}\right) \Sigma^{0}$ data bij voorwaartse hoeken [16,39]. De theorie werd echter afgeleid in het zogenaamde Regge-gebied ( $s \gg$ en $|t| \ll)$ en is als dusdanig ook enkel daar toepasbaar. Het model werd geïmplementeerd en er werd bevestigd dat de beschikbare meetgegevens in dit hoge energiegebied inderdaad goed beschreven kunnen worden. Er bestaat echter interesse om dit soort van modellen ook in het resonantie gebied toe te passen. Het feit dat de theorie een minimum aan vrije parameters bevat en volledig unitair en analytisch is, is immers een bevredigende vaststelling. Daarom werd in dit werk getracht uitbreidingen te ontwikkelen die deze extrapolatie theoretisch kunnen verantwoorden.

Een eerste vaststelling is dat Regge-theorie volledig steunt op t-kanaal uitwisselingsprocessen. Het model bevat dus geen enkele informatie over de $\mathrm{N}^{*}$ en $\Delta^{*}$ s-kanaal resonanties. Om deze toch te introduceren, werd gesteund op een model ontwikkeld door Veneziano [81,84]. Dit model stelt een correctieterm voor die kan gebruikt worden om in de Regge-beschrijving op een correcte manier s-kanaal resonanties mee te nemen. Op deze manier werd "structuur" in het resonantiegebied geïntroduceerd. De correcties voor het $\mathrm{K}^{+} \Sigma^{0}$ proces gaan in de goede richting. In het geval van de $\mathrm{K}^{+} \wedge$ schort er echter duidelijk iets aan de beschrijving en zijn de resultaten niet bevredigend.

Naast het introduceren van resonante structuur, is er ook onderzocht in hoeverre de strikte beperkingen van het Regge-gebied ( $s \gg$ en $|t| \ll)$ mathematisch kunnen worden uitgebreid. Er werd immers empirisch vastgesteld dat de Reggeamplitude ook goede resultaten geeft buiten het strikte Regge-gebied. De vooropgestelde vorm voor de Regge-amplitude is hier echter mathematisch niet meer te verantwoorden. Er werd onderzocht in hoeverre de theorie aangepast kan worden 
om aan dit probleem te verhelpen, maar tot op heden is nog geen sluitend antwoord geformuleerd. Hier is dus absoluut verder onderzoek noodzakelijk om de krachtige ideeën van Regge-theorie verder te extrapoleren en te exploiteren in het resonantie gebied. 